\title{
Encapsulation of lipids to delay lipolysis and reduce food intake
}

'From encapsulate design towards human application'

Meinou N. Corstens 


\section{Propositions}

1. Controlling lipolysis through emulsion interface design is an illusion. (this thesis)

2. To delay food digestion for a couple of hours, millennia of evolution need to be beaten. (this thesis)

3. Overeating is a waste of food that should become an integral part of food waste discussions.

4. Early education on satiation should be preferred over treating overweight later in life.

5. Just as overeating imbalances metabolism, over-publication imbalances scientific research.

6. You are what you believe you eat.

7. Leisure time drives work time efficiency.

Propositions belonging to the thesis, entitled

Encapsulation of lipids to delay lipolysis and reduce food intake

Meinou Noëlle Corstens

Wageningen, 6 April 2018 


\section{Encapsulation of lipids to delay lipolysis and reduce food intake}

'From encapsulate design towards human application'

Meinou N. Corstens 


\section{Thesis committee}

\section{Promotors}

Prof. Dr C.G.P.H. Schroën

Personal chair at the Laboratory of Food Process Engineering

Wageningen University \& Research

Prof. Dr A.A.M. Masclee

Professor of Gastroenterology and Hepatology

Maastricht University Medical Centre, University of Maastricht

\section{Co-promotors}

Dr C.C. Berton-Carabin

Assistant professor, Laboratory of Food Process Engineering

Wageningen University \& Research

Dr F.J. Troost

Associate professor Food Innovation and Health

Center of Healthy Eating and Innovation, Maastricht University campus Venlo

\section{Other members}

Prof. Dr V. Fogliano, Wageningen University \& Research

Dr K. van Norren, Wageningen University \& Research

Dr A. Meynier, INRA, Nantes, France

Dr A.T. Poortinga, Eindhoven University of Technology

This research was conducted under the auspices of the Graduate School VLAG (Advanced studies in Food Technology, Agrobiotechnology, Nutrition and Health Sciences). 


\title{
Encapsulation of lipids to delay lipolysis and reduce food intake
}

'From encapsulate design towards human application’

\author{
Meinou N. Corstens
}

Thesis

submitted in fulfilment of the requirements for the degree of doctor

at Wageningen University

by the authority of the Rector Magnificus,

Prof. Dr A.P.J. Mol,

in the presence of the

Thesis Committee appointed by the Academic Board

to be defended in public

on Friday 6 April 2018

at 1.30 p.m. in the Aula. 
Meinou N. Corstens

Encapsulation of lipids to delay lipolysis and reduce food intake, 207 pages.

PhD thesis, Wageningen University, Wageningen, the Netherlands (2018)

With references, with summaries in Dutch and English

ISBN: 978-94-6343-238-2

DOI: https://doi.org/10.18174/429534 


\section{Contents}

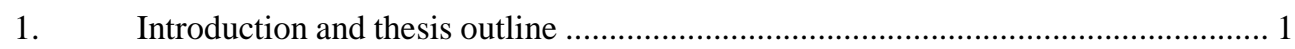

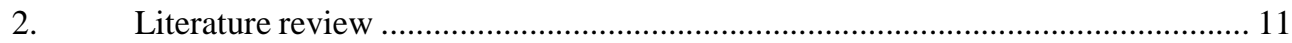

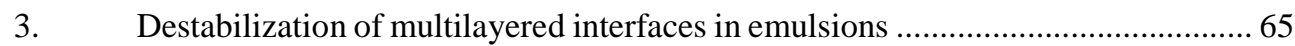

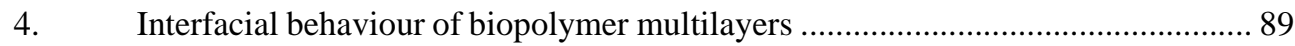

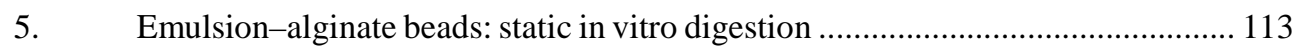

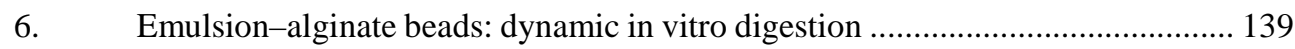

7. Emulsion-alginate beads: the effect on food intake ........................................ 163

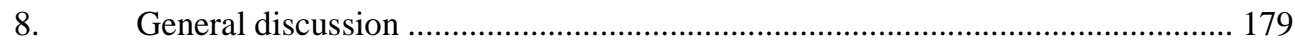

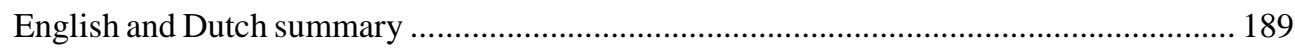

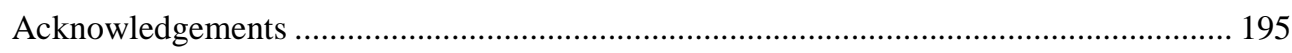

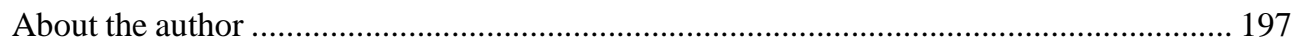





\section{Chapter 1}

Introduction and thesis outline 


\subsection{Overweight, obesity and weight-management}

According to the World Health Organisation (WHO, 2017), most individuals live in countries where overweight and obesity ${ }^{1}$ cause more deaths than underweight. Besides health consequences, overweight and obesity also come with financial consequences, not only on a personal level, but also for society as a whole. The WHO further states that overweight and obesity are largely preventable. However, that is not an easy task, and worldwide obesity has nearly tripled since 1975 .

Obesity and overweight develop as a consequence of an imbalance between energy intake and energy expenditure. At the individual level, the energy intake should be limited, the consumption of healthy foods (fruits, vegetables, legumes, whole grains and nuts) should be increased, and regular physical activity is needed (WHO, 2017). When such behavioural and life style guidelines are not effective, which is the case in an increasing number of people, the current options to help individuals to reduce their energy intake include medication (with limited efficacy and considerable side effects), endoscopic procedures, or bariatric surgery (restrictive, or combined restrictive and malabsorptive procedures) for severe cases, with excellent moderate-to-long-term efficacy (Rosenblum \& Venkatesh, 2017). However, there is an evident need for therapies that are efficacious but less invasive than bariatric surgery.

In this thesis, we employed an endogenous intestinal negative feedback mechanism, named the "ileal brake", to reduce food intake (section 1.2). To be able to do that we need to understand more about lipid digestion (section 1.3), which results in encapsulation strategies (section 1.4). These aspects lead to the research aim (section 1.5), and outline of this thesis (section 1.6).

\subsection{Ileal brake for weight-management}

A non-invasive weight-management strategy for daily use should aim at enhancing natural gastrointestinal (GI) feedback mechanisms that regulate appetite, such as gastric distension or the intestinal sensing of nutrients and its metabolites. When these are sensed by intestinal receptors in the lumen of the small intestine, gut peptides are released, such as cholecystokinin (CCK), peptide YY (PYY), and glucagon-like peptide-1 (GLP-1) (Maljaars, Peters, Mela, \& Masclee, 2008) and GI motility is inhibited, providing more time for the GI tract to digest food. These negative feedback signals increase the perception of satiety and fullness, thereby controlling food intake (Maljaars, Peters, et al., 2008). The most distal part of the small intestine, the ileum, provides the strongest satiety signal. Since these signals may

\footnotetext{
${ }^{1}$ Overweight and obesity in adults can be classified using the body mass index $\left(\mathrm{kg} / \mathrm{m}^{2}\right.$, which is indicative of weightfor-height). Overweight is defined as a BMI $\geq 25 \mathrm{~kg} / \mathrm{m}^{2}$ and obesity as a BMI $\geq 30 \mathrm{~kg} / \mathrm{m}^{2}$.
} 
result in inhibition of food intake, this phenomenon is referred to as the ileal brake (Alleleyn, van Avesaat, Troost, \& Masclee, 2016; Maljaars et al., 2011).

Previous research showed that ileal brake activation by infusing macronutrients via a nasoileal catheter directly into the human ileum can acutely reduce food intake (literature in Alleleyn et al. (2016)). For lipids, ileal infusion was shown to reduce food intake already a few decades ago (Welch, Saunders, \& Read, 1985; Welch, Sepple, \& Read, 1988), and to increase the perception of satiety/fullness (Maljaars et al., 2011; Maljaars, Symersky, et al., 2008; van Avesaat, Troost, Ripken, Hendriks, \& Masclee, 2015; Welch et al., 1985, 1988). Amongst lipids with different degree of fatty acid saturation, safflower oil (high in linoleic acid, C18:2) was found to more strongly activate the ileal brake (Maljaars, Romeyn, Haddeman, Peters, \& Masclee, 2009), which made it our lipid of choice for the research described in this thesis. The ileal brake is activated more strongly when the degradation products of lipid digestion (i.e., fatty acids and monoacylglycerols) are sensed rather than the intact lipids (i.e., triacylglycerols) themselves, as was reviewed previously by Maljaars et al. (2008).

\subsection{Digestion of lipids}

\subsubsection{Mechanism}

Lipid digestion mainly takes place by the action of different lipases and bile salts, as described in detail in Chapter 2.6. In short, lipase is the enzyme that mediates the lipolysis of triacylglycerols, the main molecular form of dietary lipids, into fatty acids and monoacylglycerols. Gastric lipase is secreted in the stomach where lipolysis starts, and pancreatic lipase in the small intestine, where lipolysis mainly occurs (Carriere, Barrowman, Verger, \& Laugier, 1993). As lipase is water-soluble and the lipid substrate is not, lipolysis occurs at the oil-water interface.

Since dietary lipids are often ingested in emulsified form (oil droplets in water: O/W emulsion), they are exposed to a large oil-water interface (McClements, 2005). Lipids can be emulsified during digestion due to the presence of bile salts in the small intestine. Bile salts are highly surface-active molecules that are able to replace interfacial material, which favours lipase adsorption and thus lipid digestion (Maldonado-Valderrama et al., 2008; Maldonado-Valderrama, Wilde, Macierzanka, \& Mackie, 2011). Besides, bile salts solubilise digestion products in mixed micelles, which enhances lipid digestion further (Sarkar, Ye, \& Singh, 2016) and enables intestinal absorption. Due to these actions, dietary lipid and its metabolites are mainly digested and already absorbed in the proximal small intestine. In the case that lipid is sensed in more distal parts of the small intestine, the GI transit needs to be slowed down to reach complete digestion and absorption, and satiety needs to be induced to 
prevent the ingestion of more food that will compete for digestive enzymes. Therefore, the strongest intestinal brake is induced from the ileum. The role of lipase and bile salts in lipolysis is depicted in Figure 1.1a.
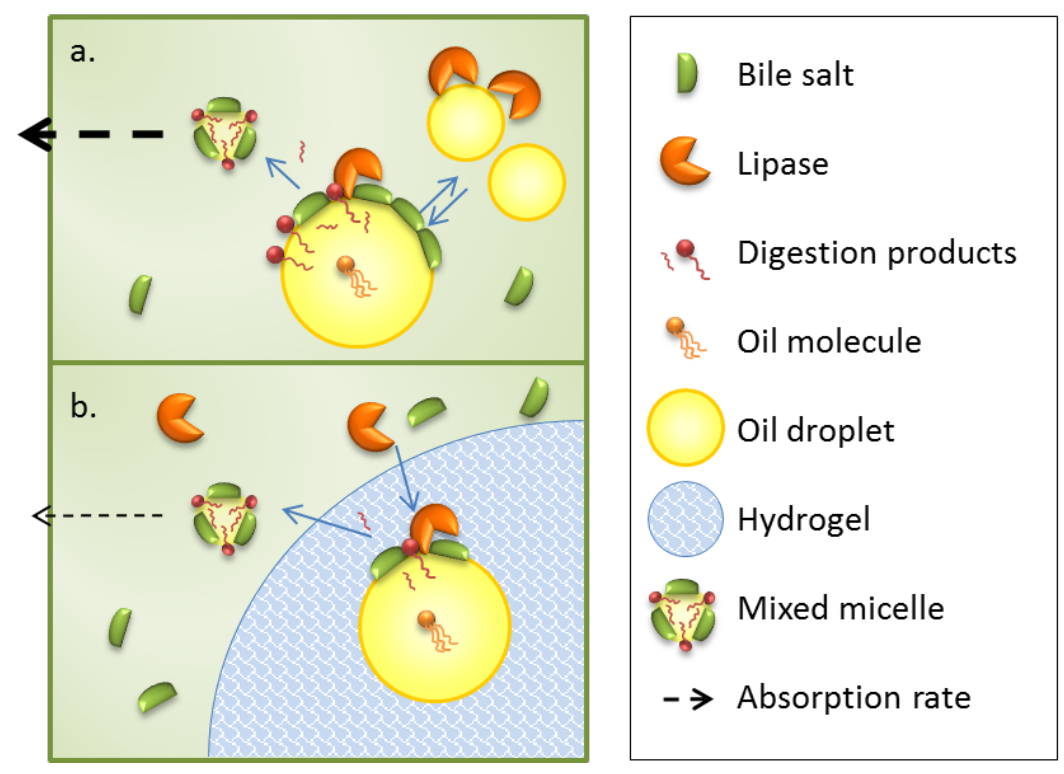

Figure 1.1. Systematic representation of the lipolytic phenomena that occur in the small intestine, due to the action of the enzyme lipase and bile salts, for a) a free emulsion and b) an emulsion entrapped in a hydrogel. The weight of the dotted arrow indicates the absorption rate.

\subsubsection{In vitro digestion measurement systems}

Human experiments to study digestion are technically demanding, and come with ethical and financial limitations, so to gain understanding of the digestive fate of foods in humans, in vitro digestion models are increasingly used (Bohn et al., 2017). In vitro models aim to mimic the in vivo situation. The most straightforward model is a static in vitro model, which is a stirred reactor that is kept at body temperature $\left(37^{\circ} \mathrm{C}\right)$ and contains a simulated digestive fluid. It should be acknowledged that the composition of simulated digestive fluids within the in vitro model is cause for extensive scientific debate, as will be discussed in more detail in Chapter 2.2.5.

Static models mostly are used, since they are cheap and easy to implement, perform and interpret, and are a good tool for screening experiments. Models that are more valid include part of the dynamics and kinetics that occur in the human body: dynamic in vitro models. A relatively easy-to-use dynamic model is the DIDGI system, which includes a series of reactors with computer-controlled transit and secretion (Ménard, Picque, \& Dupont, 2015), 
as also used in Chapter 6. On top of this, the removal of water and metabolites is included in more complex dynamic models such as the TIM system (Minekus, 2015).

\subsection{Protection of emulsion droplets}

Emulsions can become physically instable due to e.g. creaming, flocculation, coalescence and phase inversion. The rate at which this occurs depends on the interfacial properties, including composition, rheology, thickness, and charge; but also on the droplet size distribution, droplet interactions, and dispersed phase volume fraction and physical state (McClements, 2005). Physical instability changes the interfacial area and interfacial structure, so modulates digestion.

In order to reach the ileum, lipids should be protected by a 'structure' that not only survives the acidic conditions of the stomach, but also protects against the action of bile salts and lipase in the proximal small intestine. As a third important requirement, lipolysis should not be completely inhibited in the small intestine but delayed to prevent early absorption as degradation products of lipid digestion (i.e., fatty acids and monoacylglycerols) that are known to induce the ileal brake more strongly compared to intact lipids.

A number of studies attempted to design emulsion-based systems in such a way that lipolysis can be delayed and, hence, targeted lipid release is achieved. Two potential designs will be discussed: interfacial design of emulsions (1.4.1), and gelled emulsions (1.4.2).

\subsubsection{Interfacial design}

Since lipolysis occurs at the oil-water interface, the amount and accessibility of this interface is key in controlling lipolysis (Golding et al., 2011; Lundin, Golding, \& Wooster, 2008). The importance of the interface as reaction zone for lipolysis explains that many attempts have been made to control it through designing a protective interfacial structure around lipid droplets. Some of these interfacial structures were reported to delay lipolysis to a certain extent, such as monolayers of single surfactants that are claimed to provide steric hindrance, thick interfacial films produced through layer-by-layer adsorption, and colloidal particlebased interfacial structures. In Chapter 2 a review of literature is given, the main conclusion being that the high digestion efficiency of the human GI tract greatly limits the validity of this approach.

Up to now, the most promising emulsion system to deliver degradation products of lipid digestion in the distal small intestine was an emulsion of palm oil and oat oil fractions that naturally contains galactolipids, and was commercialized as Fabuless ${ }^{\circledR}$, Olibra ${ }^{\circledR}$ and Reducal ${ }^{\circledR}$. The galactolipids were expected to induce nucleation and growth of saturated fatty acids crystals, which are absorbed more slowly from the intestine (Knutson, Fridblom, 
Viberg, Sein, \& Lennerna, 2010). Unfortunately, the effect found on food intake (Smit et al., 2011) and weight management (Diepvens, Soenen, Steijns, Arnold, \& Westerterp-Plantenga, 2007) was only found for unprocessed Fabuless ${ }^{\circledR}$; it lost its functionality once subjected to food manufacturing (thermal and shear processing) (Smit et al., 2011).

\subsubsection{Entrapment of emulsion droplets within hydrogels}

In a classic $\mathrm{O} / \mathrm{W}$ emulsion, oil droplets move freely and encounter digestive components instantly in the GI tract. To delay lipid digestion, oil droplets can be trapped in a hydrogel, for which proteins and polysaccharides are commonly used in food applications (McClements, 2005). Protein solutions can be gelled by controlled cross-linking (often heatset), by controlled aggregation (forming a particle gel), or by enzymes such as transglutaminase that can be used to form covalent cross-links (McClements, 2014). Polysaccharides form a network of long molecules that are cross-linked at some places by means of (a number of) weak physical bonds called junctions (Walstra, 2003). They can be formed by temperature setting (hot or cold) or ion setting depending on the bonds between the molecules, or by specific enzymes such as laccase for sugar beet pectin (McClements, 2014). In general, polysaccharide gels are stronger, more robust, and less permeable compared to protein gels at the same concentration of gelling agent (Walstra, 2003). A gelled water or oil phase, or both, or emulsion filled-hydrogel particles in a water phase $\left(\mathrm{O} / \mathrm{W}_{1} / \mathrm{W}_{2}\right)$ can be considered, the latter option being used in this thesis.

The hydrogel not only entraps the lipid droplets, but also delays enzyme diffusion towards the oil-water interface as depicted in Figure 1.1b. Enzyme diffusion is controlled by the mesh size of the hydrogel and also by its total size. When the mesh size is wider and the total size of the hydrogel smaller, the enzyme needs less time to reach the lipid substrate (see Chapter 5). For hydrogel particles made from alginate and calcium to entrap lipids (i.e., emulsion-alginate beads), a wider mesh size (by means of lower alginate or calcium concentration) or smaller bead size was found to delay the in vitro intestinal lipolysis ( $\mathrm{Li}$, Hu, Du, Xiao, \& McClements, 2011; Zhang et al., 2016). However, it should be mentioned that the observations were not systematically linked between the digestive conditions and design of beads.

It is also known that alginate beads shrink under acidic conditions, hence lowering the mesh size (Ching, Bansal, \& Bhandari, 2015), and this effect may be used to protect the encapsulated emulsion in the stomach. In the small intestine, the higher $\mathrm{pH}$ makes the alginate hydrogel swell a little, widening the mesh size (Li et al., 2011; Rayment et al., 2009; Wright et al., 2009; Zeeb, Saberi, Weiss, \& McClements, 2015). This specific pH-dependency of the total size and permeability makes emulsion-alginate beads potential candidates to delay lipid release and, hence, ileal delivery. In the case of digestible protein hydrogels, gastric 
proteolysis causes surface erosion that controls lipolysis (Sarkar et al., 2015). Gastric proteolysis can be tuned to some extent (Dekkers, Kolodziejczyk, Acquistapace, Engmann, \& Wooster, 2016), but not completely prevented as is the case for indigestible hydrogels.

Alginate itself has been investigated in relation to its potential effect on hunger and satiety. Magnetic Resonance Imaging (MRI) has shown a prolonged residence time of alginate beads (not emulsion-loaded) in the human ileum compared to the duodenum and jejunum (Hoad et al., 2009), which suggests that there could be a stronger ileal brake effect when lipids are incorporated in the beads. Possibly this is related to the fact that alginate is a water-soluble fibre, which may delay GI transit time and result in spreading of nutrients and slower absorption of metabolites in the small intestine (Van Citters \& Lin, 2006), although no effect was found on gastric motor functions, satiation, appetite, or gut hormones when ingesting compressed alginate capsules 30 min before a meal (Odunsi et al., 2010). In a preliminary study ( $n=10$, after a fatty preload), alginate beads were shown to induce feelings of satiety depending on the hydrogel strength, but not hunger and appetite (Hoad et al., 2009). Drinks with alginate were found to incease self-reported satiety feelings depending on gelling behaviour (Peters et al., 2011) and viscosity (Solah et al., 2010). Overall, the potential direct effect of alginate in hunger modulation seems to be positive but this especially holds for alginate in solution rather than structured as bead or capsule.

Alginate beads loaded with lipid have very limitedly been studied in vivo. In rat, such beads delayed lipid absorption compared to free emulsion droplets (about 65-times lower serum levels) and emulsions clusered by an alginate matrix (about 12-times lower serum levels for $4.7 \mu \mathrm{m}$ clusers, and 5-times for $200 \mu \mathrm{m}$ clusters) (Li, Kim, Park, \& McClements, 2012). These findings were confirmed in a human trial in which oil-core alginate-shell capsules $(0.5 \mathrm{~cm})$ fastened gastric emptying, and delayed lipolysis and absorption (Hoad et al., 2011).

In summary, there are interesting positive leads, but in order to be able to design a food product that can be used in non-invasive weight management strategies, various important questions need to be answered and steps need to be made. This will be explained in the next sections.

\subsection{Research aim and approach}

The aim of this thesis was to design encapsulation systems for safflower oil that can be used as food additive, provide gastric protection and delay intestinal lipolysis such that lipolysis products are released into the distal small intestine (ileum). The work was based on the hypothesis that ileal delivery of lipids and its degradation products can activate the ileal brake mechanism to induce feelings of satiety. Our approach was multidimensional, going from the physical stability of the encapsulation systems, to their behaviour during in vitro digestion 
(static and dynamic models), and eventually towards a clinical trial to explore the effect on ad libitum food intake and satiety.

\subsection{Thesis outline}

The thesis outline is described next and a graphical representation is given in Figure 1.2. Chapter 1 introduces this thesis, which revolves around food-grade micro-encapsulation systems that may induce satiety via delayed lipolysis. Literature on these systems is reviewed in Chapter 2. In Chapter 3, we describe the production and static in vitro digestion of emulsion systems with advanced interfacial design, and demonstrate that destabilization of multilayered interfaces in digestive conditions limits their ability to prevent lipolysis in emulsions. In parallel, in Chapter 4 we explore the interfacial behaviour of these biopolymer multilayers in even more detail, including the influence of in vitro digestive conditions, using a model oil-water interface. To truly control lipolysis, emulsion-alginate beads have been designed. In Chapter 5, we focus on the production and static in vitro digestion of emulsionalginate beads; and demonstrate their ability to control in vitro intestinal lipolysis, which is a first step towards appetite-control. In Chapter 6, we evaluate the dynamic in vitro lipolysis of such emulsion-alginate beads with a DIDGI system, confirming that the time scale of lipolysis is relevant for ileal delivery, while lipolysis of a free emulsion occurs at a too high rate. In Chapter 7, we describe a randomized placebo-controlled cross-over proof of concept clinical study in which we investigated whether encapsulation of lipids as emulsion-alginate beads will reduce food intake. Finally, in Chapter 8, we provide a general discussion of the main findings and future directions.

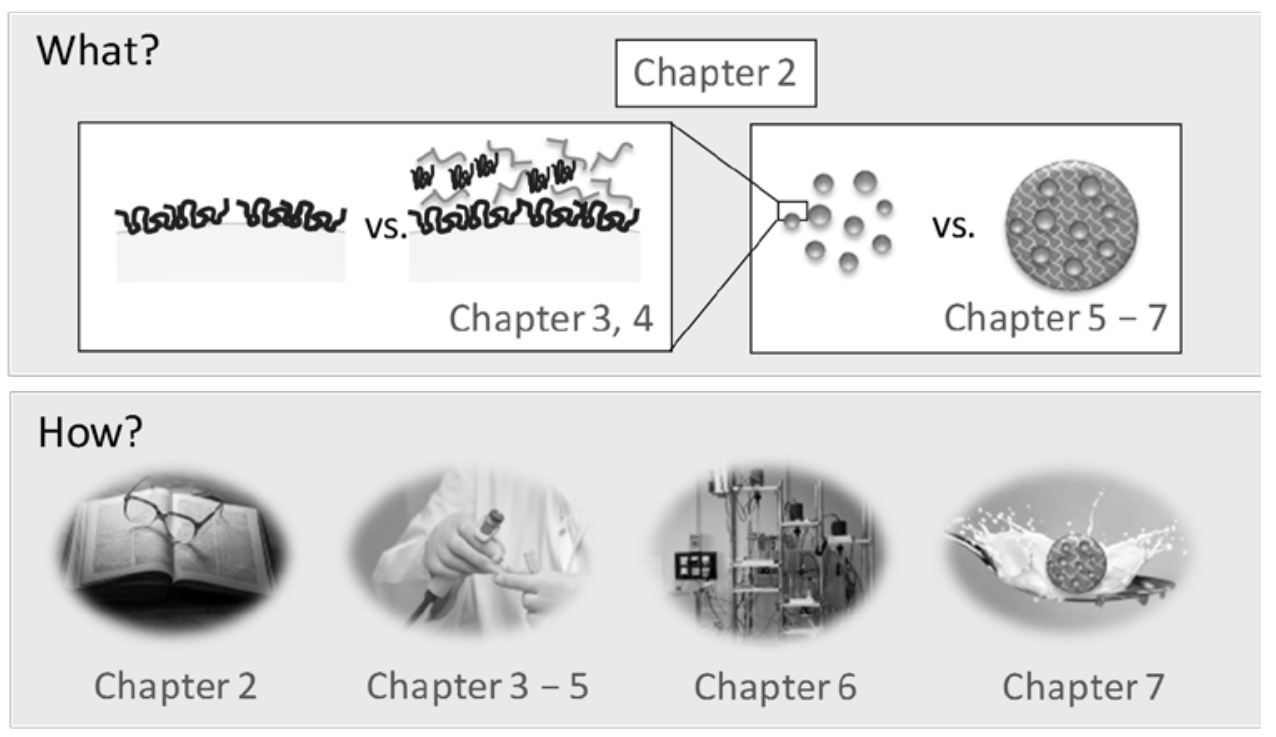

Figure 1.2. Graphical outline of the chapters of this thesis. 


\subsection{References}

Alleleyn, A. M. E., van Avesaat, M., Troost, F. J., \& Masclee, A. A. M. (2016). Gastrointestinal Nutrient Infusion Site and Eating Behavior: Evidence for A Proximal to Distal Gradient within the Small Intestine? Nutrients, 8(3), 117-132.

Bohn, T., Carriere, F., Day, L., Deglaire, A., Egger, L., Freitas, D., ... Mackie, A. R. (2017). Correlation between in vitro and in vivo data on food digestion. What can we predict with static in vitro digestion models? Critical Reviews in Food Science and Nutrition.

Carriere, F., Barrowman, J. A., Verger, R., \& Laugier, R. (1993). Secretion and contribution to lipolysis of gastric and pancreatic lipases during a test meal in humans. Gastroenterology, 105(3), 876-888.

Ching, S. H., Bansal, N., \& Bhandari, B. (2015). Alginate gel particles- A review of production techniques and physical properties. Critical Reviews in Food Science and Nutrition, 57(6), 1133-52.

Dekkers, B., Kolodziejczyk, E., Acquistapace, S., Engmann, J., \& Wooster, T. J. (2016). Impact of gastric $\mathrm{pH}$ profiles on the proteolytic digestion of mixed $\beta 1 \mathrm{lg}$-Xanthan biopolymer gels. Food Function, 7(1), 58-68.

Diepvens, K., Soenen, S., Steijns, J., Arnold, M., \& Westerterp-Plantenga, M. (2007). Long-term effects of consumption of a novel fat emulsion in relation to body-weight management. International Journal of Obesity, 31(6), 942-9.

Golding, M., Wooster, T. J., Day, L., Xu, M., Lundin, L., Keogh, J., \& Clifton, P. (2011). Impact of gastric structuring on the lipolysis of emulsified lipids. Soft Matter, 7, 3513-3523.

Hoad, C., Rayment, P., Cox, E., Wright, P., Butler, M., Spiller, R., \& Gowland, P. (2009). Investigation of alginate beads for gastro-intestinal functionality, Part 2: In vivo characterisation. Food Hydrocolloids, 23(3), 833-839.

Hoad, C., Rayment, P., Risse, V., Cox, E., Ciampi, E., Pregent, S., ... Gowland, P. (2011). Encapsulation of lipid by alginate beads reduces bio-accessibility: An in vivo 13C breath test and MRI study. Food Hydrocolloids, 25(5), 1190-1200.

Knutson, L., Fridblom, H., Viberg, A., Sein, A., \& Lennerna, H. (2010). Gastrointestinal metabolism of a vegetable-oil emulsion in healthy. American Journal of Clinical Nutrition, 92, 515-524.

Li, Y., Hu, M., Du, Y., Xiao, H., \& McClements, D. J. (2011). Control of lipase digestibility of emulsified lipids by encapsulation within calcium alginate beads. Food Hydrocolloids, 25(1), 122-130.

Li, Y., Kim, J., Park, Y., \& McClements, D. J. (2012). Modulation of lipid digestibility using structured emulsion-based delivery systems: comparison of in vivo and in vitro measurements. Food \& Function, 3(5), 528-36.

Lundin, L., Golding, M., \& Wooster, T. J. (2008). Understanding food structure and function in developing food for appetite control. Nutrition and Dietetics, 65(Suppl. 3), S79-85.

Maldonado-Valderrama, J., Wilde, P., Macierzanka, A., \& Mackie, A. (2011). The role of bile salts in digestion. Advances in Colloid and Interface Science, 165, 36-46.

Maldonado-Valderrama, J., Woodward, N. C., Patrick Gunning, A., Ridout, M. J., Husband, F. A., Mackie, A. R., ... Wilde, P. J. (2008). Interfacial characterization of B-lactoglobulin networks: Displacement by bile salts. Langmuir, 24(13), 6759-6767.

Maljaars, P. W. J., Peters, H. P. F., Kodde, A., Geraedts, M., Troost, F. J., Haddeman, E., \& Masclee, A. A. M. (2011). Length and site of the small intestine exposed to fat influences hunger and food intake. The British Journal of Nutrition, 106(10), 1609-15.

Maljaars, P. W. J., Peters, H. P. F., Mela, D. J., \& Masclee, A. A. M. (2008). Ileal brake: a sensible food target for appetite control. A review. Physiology \& Behavior, 95(3), 271-281.

Maljaars, P. W. J., Romeyn, E. A., Haddeman, E., Peters, H. P. F., \& Masclee, A. A. M. (2009). Effect of fat saturation on satiety, hormone release, and food intake. American Journal of Clinical Nutrition, 89, 1019-1024.

Maljaars, P. W. J., Symersky, T., Kee, B. C., Haddeman, E., Peters, H. P. F., \& Masclee, A. A. M. (2008). Effect of ileal fat perfusion on satiety and hormone release in healthy volunteers. International Journal of Obesity, 32(11), 1633-9.

McClements, D. J. (2005). Food Emulsions: Principles, Practices, and Techniques. (F. M. Clydesdale, Ed.) (Second). CRC Press.

McClements, D. J. (2014). Nanoparticle- and Microparticle-based Delivery Systems. CRC Press.

Ménard, O., Picque, D., \& Dupont, D. (2015). The DIDGI System. In L.-E. I. et al Verhoeckx K, Cotter P (Ed.), The Impact of Food Bioactives on Health (pp. 73-81). New York: Springer International Publishing. 
Minekus, M. (2015). The TNO Gastro-Intestinal Model (TIM). In L.-E. I. et al Verhoeckx K, Cotter P (Ed.), The Impact of Food Bioactives on Health (pp. 37-46). New York: Springer International Publishing.

Odunsi, S. T., Vázquez-Roque, M. I., Camilleri, M., Papathanasopoulos, A., Clark, M. M., Wodrich, L., ... Zinsmeister, A. R. (2010). Effect of alginate on satiation, appetite, gastric function, and selected gut satiety hormones in overweight and obesity. Obesity (Silver Spring, Md.), 18(8), 1579-1584.

Peters, H. P. F., Koppert, R. J., Boers, H. M., Ström, A., Melnikov, S. M., Haddeman, E., ... Wiseman, S. A. (2011). Dose-dependent suppression of hunger by a specific alginate in a low-viscosity drink formulation. Obesity (Silver Spring, Md.), 19(6), 1171-1176.

Rayment, P., Wright, P., Hoad, C., Ciampi, E., Haydock, D., Gowland, P., \& Butler, M. F. (2009). Investigation of alginate beads for gastro-intestinal functionality, Part 1: In vitro characterisation. Food Hydrocolloids, 23(3), 816-822.

Rosenblum, J., \& Venkatesh, R. D. (2017). Obesity. In M. A. Goldstein (Ed.), The MassGeneral Hospital for Children Adolescent Medicine Handbook (pp. 67-75). Cham: Springer International Publishing.

Sarkar, A., Juan, J.-M., Kolodziejczyk, E., Acquistapace, S., Donato-Capel, L., \& Wooster, T. J. (2015). Impact of protein gel porosity on the digestion of lipid emulsions. Journal of Agricultural and Food Chemistry, 63(40), 8829-8837.

Sarkar, A., Ye, A., \& Singh, H. (2016). On the role of bile salts in the digestion of emulsified lipids. Food Hydrocolloids, 60, 77-84.

Smit, H. J., Keenan, E., Kovacs, E. M. R., Wiseman, S. a, Peters, H. P. F., Mela, D. J., \& Rogers, P. J. (2011). No efficacy of processed Fabuless (Olibra) in suppressing appetite or food intake. European Journal of Clinical Nutrition, 65(1), 81-6.

Solah, V. A., Kerr, D. A., Adikara, C. D., Meng, X., Binns, C. W., Zhu, K., ... Prince, R. L. (2010). Differences in satiety effects of alginate- and whey protein-based foods. Appetite, 54(3), 485-491.

van Avesaat, M., Troost, F. J., Ripken, D., Hendriks, H. F., \& Masclee, A. A. M. (2015). Ileal brake activation: macronutrient specific effects on eating behavior? International Journal of Obesity, 39(April), 235243.

Van Citters, G. W., \& Lin, H. C. (2006). Ileal brake: neuropeptidergic control of intestinal transit. Current Gastroenterology Reports, 8(5), 367-73.

Walstra, P. (2003). Physical Chemistry of Foods.

Welch, I., Saunders, K., \& Read, N. W. (1985). Effect of ileal and intravenous infusions of fat emulsions on feeding and satiety in human volunteers. Gastroenterology, 89(6), 1293-1307.

Welch, I., Sepple, C. P., \& Read, N. W. (1988). Comparisons of the effects on satiety and eating behaviour of infusion of lipid into the different regions of the small intestine. Gut, 29, 306-311.

WHO. (2017). Obesity and overweight. Retrieved from http://www.who.int/mediacentre/factsheets/fs311/en/

Wright, P. J., Ciampi, E., Hoad, C. L., Weaver, A. C., van Ginkel, M., Marciani, L., ... Rayment, P. (2009). Investigation of alginate gel inhomogeneity in simulated gastro-intestinal conditions using magnetic resonance imaging and transmission electron microscopy. Carbohydrate Polymers, 77(2), 306-315.

Zeeb, B., Saberi, A. H., Weiss, J., \& McClements, D. J. (2015). Retention and release of oil-in-water emulsions from filled hydrogel beads composed of calcium alginate: impact of emulsifier type and $\mathrm{pH}$. Soft Matter, 11, 2228-2236.

Zhang, Z., Zhang, R., Zou, L., Chen, L., Ahmed, Y., Bishri, W. Al, .. McClements, D. J. (2016). Encapsulation of curcumin in polysaccharide-based hydrogel beads: Impact of bead type on lipid digestion and curcumin bioaccessibility. Food Hydrocolloids, 58, 160-170. 


\section{Chapter 2}

Literature review

This chapter has been published as Corstens, M.N., Berton-Carabin, C.C., de Vries, R., Troost, F.J., Masclee, A.A.M., \& Schroën, K. (2017) Food-grade Micro-encapsulation Systems that May Induce Satiety via Delayed Lipolysis: A Review. Critical Reviews in Food Science and Nutrition 57(10):2218-2244. 


\section{ABSTRACT}

The increasing prevalence of overweight and obesity requires new, effective prevention and treatment strategies. One approach to reduce energy intake is by developing novel foods with increased satiating properties, which may be accomplished by slowing down lipolysis to deliver substrates to the ileum, thereby enhancing natural gut-brain signalling pathways of satiety that are normally induced by meal intake.

To develop slow release food additives, their processing in the gastrointestinal tract has to be understood; therefore, we start from a general description of the digestive system and relate that to in vitro modelling, satiety and lipolytic mechanisms. The effects of physicochemical lipid composition, encapsulation matrix and interfacial structure on lipolysis are emphasized. We give an overview of techniques and materials used, and discuss partitioning, which may be a key factor for encapsulation performance.

Targeted release capsules that delay lipolysis form a real challenge because of the high efficiency of the digestive system; hardly any proof was found that intact orally ingested lipids can be released in the ileum and thereby induce satiety. We expect that this challenge could be tackled with structured $\mathrm{O} / \mathrm{W}$ emulsion-based systems that have some protection against lipase, e.g., by hindering bile salt adsorption and/or delaying lipase diffusion. 


\subsection{INTRODUCTION}

Since 1980, worldwide obesity prevalence has nearly doubled according to World Health Organization statistics (WHO 2014). Two thirds of the world's population live in countries where overweight and obesity results in higher morbidity and mortality compared to being underweight. The increasing number of overweight and obese people indicates that energy intake and energy expenditure are not balanced. Interestingly, the WHO key facts also state that "obesity is preventable”. The intake of energy could be reduced by making products more satiating, and one approach to achieve this is to slow down digestion, which results in the presence of intact nutrients in more distal parts of the gastrointestinal (GI) tract. This in turn can activate the so-called ileal brake, which induces satiety via hormonal and neural signalling routes and thereby reduces the food intake (Maljaars et al. 2008a, van Avesaat et al. 2015). When dietary triacylglycerols reach the ileum without being digested, they are able to activate this natural feedback mechanism to induce satiety. However, it should be taken into account that it is not easy to achieve delivery of intact orally ingested nutrients to more distal parts of the GI tract due to the high efficiency of the digestive processes. Hence, studies to the ileal brake principle in vivo in humans are at present limited mainly to complex intubation studies.

The present review focuses on how to control lipolysis under physiological conditions. Dietary fat digestion is determined by the physicochemical composition of the lipid and the interfacial area between lipid and the surrounding environment. Encapsulation of the lipid material is expected to help controlling lipolysis, hence this review focuses on encapsulates, their interfacial structure, and composition in relation to digestion.

Over the past century, encapsulation systems that can protect certain ingredients (e.g., antioxidants, colorants) during storage were designed to improve the products' shelf-life (Gibbs et al. 1999). Over the last decades, the application of food encapsulation systems has shifted towards controlled delivery in the human digestion system, for example by providing protection against the gastric environment for vitamins, or delivering drugs or probiotics to the colon. Thus, beyond stability during storage, the capsule must provide appropriate stability against the disruptive conditions in the human body.

In the medical field, numerous encapsulation systems are available for controlled delivery of drugs via oral administration, or to improve their bioavailability (Lam \& Gambari 2014). However, the materials and techniques used to produce such encapsulates in pharmacy are often not suitable for food applications, as they are not food-grade. Besides, they are often too expensive to be used as food ingredients. Yet, the knowledge on technologies and materials from the medical field is a very sound basis to design food-grade systems that need comparable properties. 
In depth understanding of the physiological conditions and digestion processes is required to design encapsulation systems for delayed lipolysis in the human GI tract. Reversed engineering can be used to obtain optimal functionality, which focuses on the application, rather than looking for an application after designing an encapsulation system from a technological point of view (Cerqueira et al. 2013). A smart design is crucial for success, because the capsule faces different harsh disruptive environments in the GI tract, as the digestion system has evolved over millions of years towards efficient food processing. In order to achieve controlled release of micro-encapsulated ingredients in the GI tract, these digestive factors - such as $\mathrm{pH}$, electrolyte balance, bile salt composition, temperature, and mechanical stress - have to be counteracted when the capsule needs to be stable or can be used as trigger for release. According to Benshitrit et al., the future challenges in developing oral food-grade delivery systems are i) the structure-function relationships, particularly the fate of delivery systems, ii) the sensory properties, and iii) the human variance and complexity of the digestive process (Benshitrit et al. 2012).

The present review aims at combining physiology and technology insights to highlight the important parameters that should be considered when developing micro-encapsulates for delayed lipolysis in digestive conditions. Relevant physiological and technological insights are first discussed, before we discuss in section 2.6 the available research on ways to delay lipolysis and hence increase feelings of satiety. The physiological conditions in the different parts of the human GI tract will first be described together with existing in vitro models, satiety and digestion of lipids. Next, available micro-encapsulation methods are described, as well as suitable materials for lipid encapsulation, with the focus on food-grade options. The dynamic partitioning of ingredients among the available phases of micro-encapsulation systems will be discussed, since it may strongly influence the performance of encapsulates. A summary table of common encapsulation materials, encapsulated ingredients, release triggers and encapsulation methods/techniques is presented in Figure 2.1, and will be discussed into greater detail in the review. 


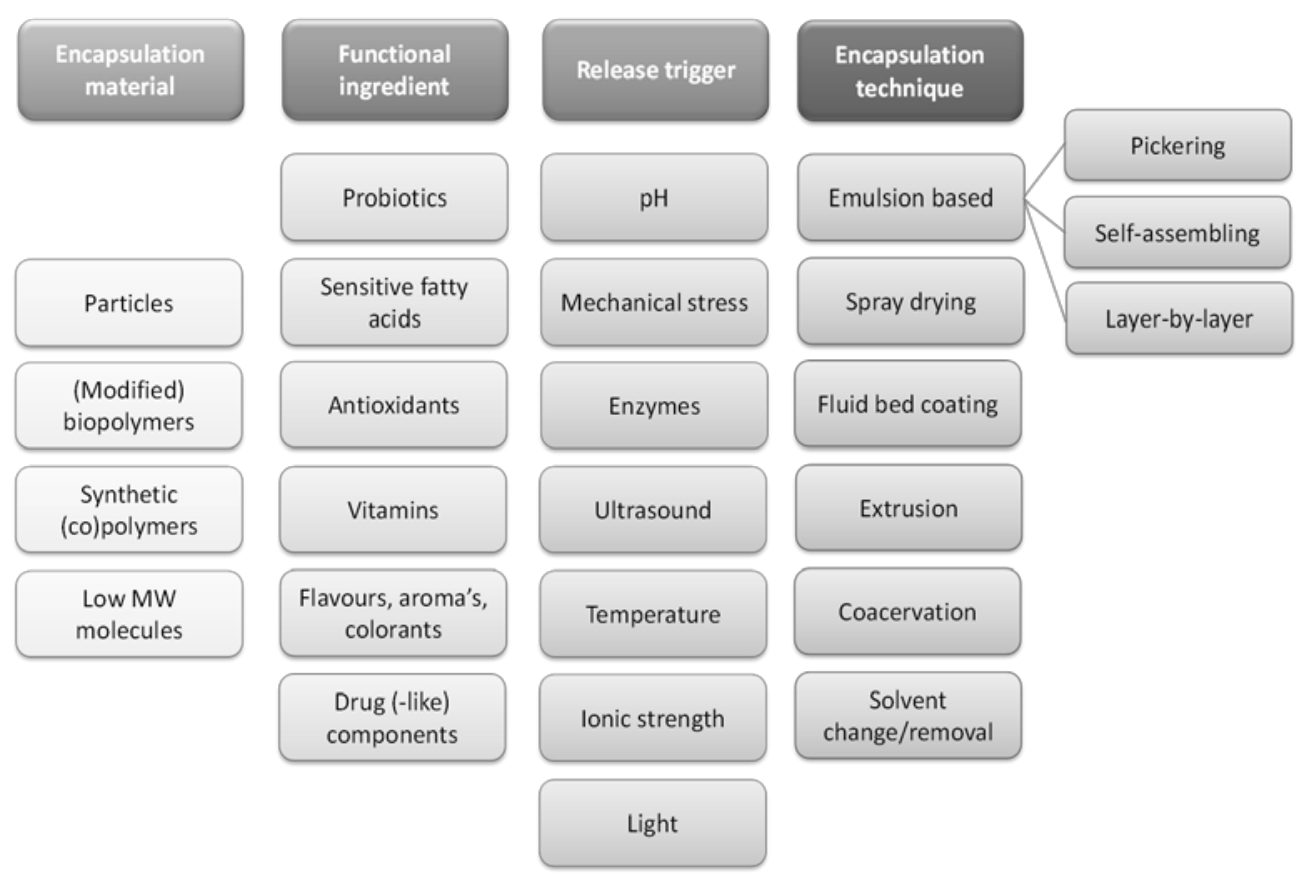

Figure 2.1. Overview of materials and techniques to create micro-encapsulation systems, encapsulated functional ingredients and release triggers.

\subsection{THE HUMAN DIGESTIVE SYSTEM}

McConnell and co-workers concluded, after critically reviewing the intestinal physiology parameters, that there is no such a thing as an 'average person' (McConnell et al. 2008). However, gastrointestinal (GI) food processing occurs through some general principles (Figure 2.2; for dimensions see Table 2.1). Below we first discuss the physiological conditions in the different compartments, followed by in vitro modelling.

\subsubsection{Mouth and oesophagus}

Consumed food will be partially masticated by the teeth, and mixed with saliva in the oral cavity. Saliva contains electrolytes, carbohydrate degrading enzymes (amylase) and biopolymers (e.g., mucin). Mucins are mucosal glycoproteins (polypeptide backbone, with oligosaccharide side chains), present at concentrations of about $30-500 \mu \mathrm{g} \cdot \mathrm{ml}^{-1}$ saliva (Vingerhoeds et al. 2005). Although their solubility in water is limited, the oligosaccharide chains of mucins get readily hydrated and have high water binding capacity, which increases the viscosity and elasticity of saliva, resulting in lubrication of the food bolus (Minekus et al. 2014). Mucins can cause aggregation of most food emulsions; the destabilization mechanism 
depends on the charge of the emulsion droplet. Weakly negatively charged to neutral emulsions most likely undergo depletion flocculation by mucins, while positively charged emulsions show bridging flocculation (van Aken et al. 2007). Solid food also undergoes chewing (mastication), which reduces the size of food particles. The food components can interact with the tongue and mouth surface, and the flow in the mouth can be complex, especially for liquid foods.

After swallowing, the formed bolus enters in the oesophagus, with a $\mathrm{pH}$ of about 5-6 in the lumen. The bolus is transported through this muscular $25 \mathrm{~cm}$ long tube with a diameter approximately $2 \mathrm{~cm}$, by swallowing in a single peristaltic wave of contraction, towards the stomach.

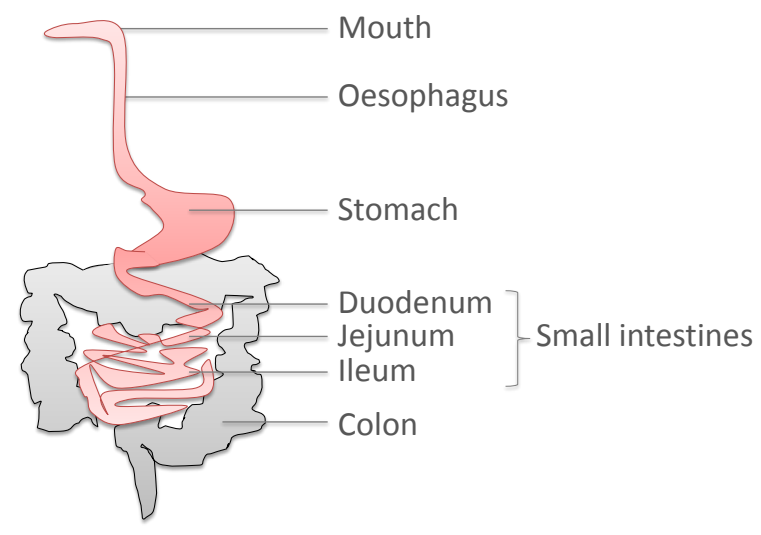

Figure 2.2. Schematic representation of the human digestive tract.

\subsubsection{Stomach}

In the stomach, food processing is continued by the action of enzymes and hydrochloric acid. In order to protect the cells of the stomach wall against the destructive lumen content, viscous mucus is secreted by goblet cells that remains on top of the gastric cells where the mucus forms a firmly adherent gel layer, onto which also a looser mucus layer resides (Atuma et al. 2001). The stomach is in general acidic due to the secretion of hydrochloric acid by parietal cells, but varies greatly between individuals and within a person over time. Intra-individual variations in stomach acidity is caused by several factors such as dietary intake, seasonal variations, composition of the previous meal, feeding status, health status, age, etc. 
Table 2.1. Compartments of the human digestive tract and their main characteristics. Adapted from Kenmogne-Domguia (2012), with permission of H. B. Kenmogne-Domguia.

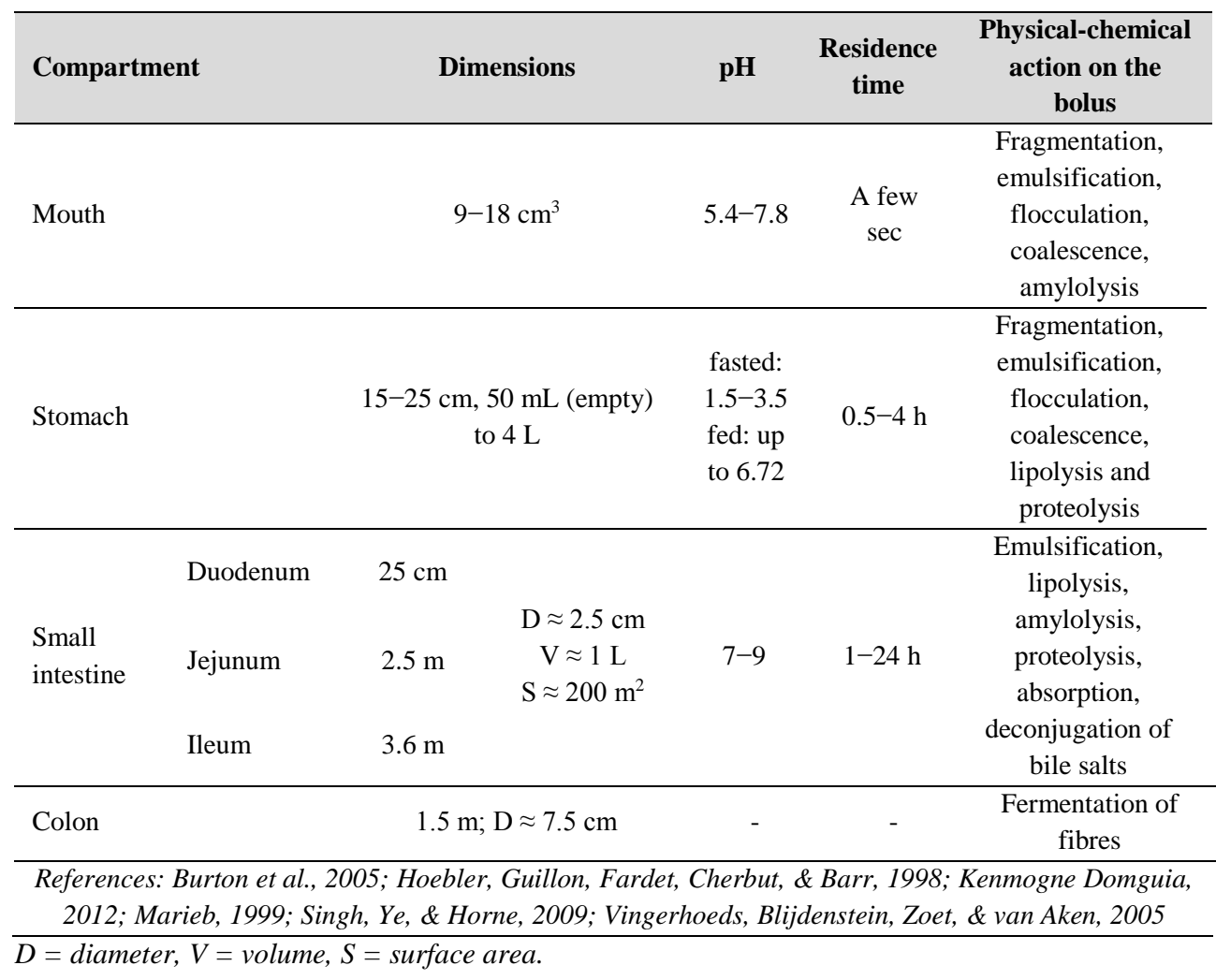

In general, the stomach in the fasted state - where no food is present - is more acidic than in fed state, but various absolute $\mathrm{pH}$ values have been reported. Also the health status can affect this $\mathrm{pH}$. It has been shown that, for example, obese people had a much lower $\mathrm{pH}$ than nonobese subjects (1.7 versus 3.7) in fasted state (Vaughan et al. 1973).

The presence of nutrients in the stomach induces the release of gastrin by the G-cells in the stomach wall. Gastrin is a hormone that stimulates the production of acid and enzymes, and affects the motility of the stomach. Stomach epithelium produces and secretes several digestive enzymes (Moreau et al. 1988); chief cells secrete pepsinogen that is the precursor of pepsin, which breaks down proteins and is active at $\mathrm{pH}$ values up to 5 , above which it is inactivated. Chief cells can also produce gastric lipase, a lipolytic enzyme that is stable and active at acidic pH (Bakala N'Goma et al. 2012).

The stomach generates peristaltic contractions towards the end of the stomach (pylorus), which induces intra-gastric flow, with an average speed of $1.5-3 \mathrm{~mm} \cdot \mathrm{s}^{-1}$ for the antral contraction waves, with a frequency of $2.6-3$ waves $\cdot \mathrm{min}^{-1}$ (Bornhorst \& Singh 2014). 
Although there is no consensus about the exact effect of meal composition and the interaction between consumer and food properties, the flow patterns have been shown to depend on certain food properties such as viscosity, density and particle size (Bornhorst \& Singh 2014). The overall direction of flow of liquid materials is opposite to the direction of peristalsis and the shear rate profile is described to be hardly affected by the viscosity of liquid phases (Kozu et al. 2014). Solid meals require more than just mixing with gastric juice, they have to be broken before leaving the stomach, so antral grinding is an important process (Marciani et al. 2001). Softer materials have been shown by Marciani et al. to be broken more rapid and to empty faster than harder materials, like liquids.

In addition to mixing, the stomach functions as a compartment to temporarily store ingested food with a variable residence time (between $5 \mathrm{~min}$ (McConnell et al. 2008) and $5 \mathrm{~h}$ (Lesmes \& McClements 2009). The gastric emptying rate determines the residence time in the gastric fluid, and controls the speed at which the chyme enters the intestines; fluids and small particles have short residence times, while bigger particles are kept longer to ensure better digestion. The gastric emptying rate directly relates to the caloric content (Calbet \& MacLean 1997), and is also dependent on meal composition: for instance fried pasta delayed the emptying as compared to boiled pasta, and a brown rice meal lead to slower emptying than white rice (Bornhorst \& Singh 2014). In addition, the order of nutrient ingestion can affect the emptying rate, it being faster when non-fat components are served before fat components (Kunz et al. 2005). Besides the texture and composition of the food, the emptying rate is also regulated via several feedback mechanisms (van Aken 2010), for example the presence of residual nutrients in the intestines from the previous meal slows it down, and the gut hormone levels of cholecystokinin (CCK), peptide YY (PYY) and glucagon-like peptide-1 (GLP-1) can also modulate it. Olsson \& Holmgren stated that "almost everything seems to affect gastric emptying” (Olsson \& Holmgren 2001).

\subsubsection{Small intestine}

The small intestine can be considered as a 6-7 meter long tube of which the inside is called the lumen (diameter about $2.5 \mathrm{~cm}$ ). It can be divided into three zones; the most proximal part is called duodenum, the middle region the jejunum and most distal part the ileum. The stomach content (chyme) is slowly emptied into the lumen of the duodenum, and consists then of a blend of partly digested food and secreted digestion fluid. The overall residence time in the small intestine is 0.5 to $9.5 \mathrm{~h}$, depending on the nutrient composition, and on the physical and chemical structure of the chyme (Coupe et al. 1991); the variation within individual subjects was even significant when repetitively providing the same meal. 
During the duodenal phase, secretions are added from the pancreas, gallbladder and intestinal wall. The pancreas secretes a range of digestive enzymes, including proteases (trypsin and chymotrypsin), esterase, lipase, phospholipase and amylase. Most of these enzymes are secreted as inactive pro-enzymes, and are activated after being secreted into the lumen of the small intestine (van Aken 2010). The gallbladder secretes bicarbonate and bile. Bicarbonate neutralizes the acidic chyme to a $\mathrm{pH}$ of about 5.5 in the duodenum, and to about 7.5 when reaching the ileum. Large variations in $\mathrm{pH}$ profiles between individuals have been measured, and also within people under the same feeding conditions (Ibekwe et al. 2008). Bile contains bile salts and phospholipids, which are essential for the digestion and absorption of components with low water-solubility; below their role is described in more detail.

The intestinal wall is structured with villi, finger-shaped outgrowths, to provide a large surface area containing the epithelial cells that absorb the digestion products into the blood stream. The epithelial cells, or enterocytes, form a single cell layer covering the small intestine. The enterocytes express microvilli, small protrusions into the intestinal lumen. They increase the cells surface area in the lumen and form the brush border, or glycocalyx, which composes of glycoproteins and digestive enzymes. The villi form the innermost mucosa layer, which is positioned onto the submucosa that contains blood vessels, lymphatic vessels and nerves, that all work together to allow for uptake of the digestion products.

Peristaltic movements are generated by circular and longitudinal muscle layers that surround the mucosa - submucosa. As a result of this peristalsis, the food and digestive components are mixed and moved through the small intestine. In between the epithelial cells, some enteroendocrine and goblet cells are present. Enteroendocrine cells release peptide hormones based on the present nutrients during and after a meal, including CCK, GLP-1 and PYY which are described into more detail in section 3.1. Goblet cells secrete a mucous layer that protects the epithelia. Mucus consists mainly of water, with some salts, proteins and the glycoprotein mucin. In contrast to the stomach, only a thin mucus layer is firmly adherent here, and also the loosely adhered layer on top is thinner (Atuma et al. 2001). This allows permeation of nutrients and facilitates absorption (van Aken 2010). Food polymers can attach to the mucosa on the intestinal wall, called mucoadhesion, when they are capable of taking up water from the mucus via hydration. The adhesion strength has been shown to depend on the properties of the polymer, such as molecular composition and the actual pH (Grabovac et al. 2005).

\subsubsection{Colon}

After the ileum, the food reaches the colon, which is approximately 1.5 meter long, and also called the large intestine because of its larger diameter of about $7.5 \mathrm{~cm}$. The main function 
of the colon is to allow microbial fermentation of unabsorbed nutrients. Accordingly, one gram of intestinal content will hold about $10^{11}-10^{12}$ anaerobic microorganisms (Simon \& Gorbach 1986). A thick mucus layer protects the mucosal cells of the colon wall against the lumen content. This layer consists of $120 \mu \mathrm{m}$ thick, firmly adherent mucus to the wall, and about $700 \mu \mathrm{m}$ thick loosely adhered mucus on top (Atuma et al. 2001). Peristaltic contractions and segmentation induce movement through the colon, and finally excretion as faeces. The colon does not play an important role in lipolysis. It is worth mentioning that targeted delivery in the colon has been reported, for example of probiotics (Dong et al. 2013), which requires the use of encapsulation systems that resist digestion and absorption in earlier stages (Situ et al. 2014).

\subsubsection{In vitro modelling}

In model systems, theoretically it would be possible to reproduce all conditions involved in human digestion; however, their relative effects and importance are not completely unravelled yet. Moreover, in practice it is not possible to take all factors into account in one model. Therefore, simplified models are used that only include those factors that are assumed to have a major influence on the investigated system.

It should be pointed out that a broad range of in vitro digestion models have been used, with just one factor being the same over all studies: a temperature of $37^{\circ} \mathrm{C}$. Due to these nonharmonized in vitro digestion models, the comparability across research teams was not assured (Hur et al. 2011, Li et al. 2011, Marze et al. 2012, McClements \& Li 2010a, Porter \& Charman 2001). Recently, Minekus et al. published an international consensus on a standardized method for static in vitro digestion for food (Minekus et al. 2014). It is a promising initiative since the article gives detailed guidelines for the protocol, such as the concentrations of the stock solutions and the dilution factor to be used, and the authors are recognized scientists in the field. But, the described method is a static model, so does not include the mechanical forces and dynamic processes in the human body.

In vitro model conditions that can be used to represent these above described compartments are concurrently presented and discussed below.

\section{Oral phase}

For liquid meals, the in vitro digestion mostly excludes the oral and oesophagus steps, but when starch is present a simulated salivary fluid (SSF) is sometimes used. Conversely, for solid meals the oral phase cannot be excluded from the in vitro digestion model, and in addition to the SSF, it needs to induce a mincing step. Commercial mincers are available, which mimic the mechanical forces of chewing. The saliva simulation includes two min mixing liquid food with a volume ratio of $1: 1$ to SFF ( $\mathrm{pH}$ of 7 , fixed electrolyte concentrations 
$\left(18.8 \mathrm{mmol} \cdot \mathrm{L}^{-1} \mathrm{~K}^{+} ; 13.6 \mathrm{mmol} \cdot \mathrm{L}^{-1} \mathrm{Na}^{+} ; 19.5 \mathrm{mmol} \cdot \mathrm{L}^{-1} \mathrm{Cl}^{-} ; 3.7 \mathrm{mmol} \cdot \mathrm{L}^{-1} \mathrm{H}_{2} \mathrm{PO}_{4}^{-}\right.$; $13.7 \mathrm{mmol} \cdot \mathrm{L}^{-1} \mathrm{HCO}_{3}{ }^{-}, \mathrm{CO}_{3}{ }^{2-} ; 0.15 \mathrm{mmol} \cdot \mathrm{L}^{-1} \mathrm{Mg}^{2+} ; 0.12 \mathrm{mmol} \cdot \mathrm{L}^{-1} \mathrm{NH}^{4+} ; 1.5 \mathrm{mmol} \cdot \mathrm{L}^{-1} \mathrm{Ca}^{2+}$ ), amylase (75 $\mathrm{U} \cdot \mathrm{mL}^{-1}$ ) (Minekus et al. 2014). The effect of mucin is hard to mimic in vitro, due to large variations between and within individuals (Vingerhoeds et al. 2005); therefore, Minekus et al. (2014) also advice not to use it in standardized in vitro digestion.

\section{Gastric phase}

As described in the paragraph 0 , the gastric digestion is a complex and variable system, which is hard to study and, therefore, to model (Bornhorst \& Singh 2014). Hence, it is often unclear which factors have to be included. In the widely used static models, the gastric motility, gastric secretions over time and gastric emptying rate are not included, and the sample is simply incubated in a stirred simulated gastric fluid (SGF) at $37^{\circ} \mathrm{C}$, generally for $2 \mathrm{~h}$ ). The advised composition includes a $\mathrm{pH}$ of 3 , pepsin $\left(2,000 \mathrm{U} \cdot \mathrm{mL}^{-1}\right)$, fixed electrolyte concentrations $\left(7.8 \mathrm{mmol} \cdot \mathrm{L}^{-1} \mathrm{~K}^{+} ; 72.2 \mathrm{mmol} \cdot \mathrm{L}^{-1} \mathrm{Na}^{+} ; 70.2 \mathrm{mmol} \cdot \mathrm{L}^{-1} \mathrm{Cl}^{-} ; 0.9 \mathrm{mmol} \cdot \mathrm{L}^{-1}\right.$ $\mathrm{H}_{2} \mathrm{PO}_{4}^{-} ; 25.5 \mathrm{mmol} \cdot \mathrm{L}^{-1} \mathrm{HCO}_{3}{ }^{-}, \mathrm{CO}_{3}{ }^{2-} ; 0.1 \mathrm{mmol} \cdot \mathrm{L}^{-1} \mathrm{Mg}^{2+} ; 1.0 \mathrm{mmol} \cdot \mathrm{L}^{-1} \mathrm{NH}^{4+} ; 0.15 \mathrm{mmol} \cdot \mathrm{L}^{-1}$ $\mathrm{Ca}^{2+}$ ) and in case the sample does not contain low molecular weight surfactants also $0.17 \mathrm{mM}$ of the phospholipid egg lecithin should be included (Minekus et al. 2014).

Gastric lipolysis has mostly been omitted in in vitro digestion so far, due to the absence of commercial gastric lipase substitute for acceptable costs and besides, the experts are not fully convinced about the exact role of human gastric lipase (Bakala N'Goma et al. 2012, Minekus et al. 2014). However, the use of human gastric fluid for in vitro digestion showed greater lipid hydrolysis than in a simulated fluid with artificial enzymes, although the proteolytic and lipolytic activity was at the same level (Malinauskyte et al. 2014). The translation of results obtained in model systems to physiological conditions may be hampered because of this.

Obviously, the most relevant processes need to be included in model systems; however this is not as straightforward as it may sound. In static models, the impact of mechanical force, liquid flow, shear stress, dilutions by gastric secretions over time and gastric emptying is not taken into account. For some research questions this is not problematic, but for others it cannot be neglected. Especially when structural aspects of the solid food matrixes are involved, it is crucial to include the dynamics in the model. Gastric motility has been studied by techniques such as Magnetic Resonance Imaging (Kunz et al. 2005), manometry and with the use of wireless capsules, and based on that, model systems were build. Examples of dynamic gastric in vitro models are the TNO Gastro-Intestinal Model (TIM-1) (Minekus 2015), the 'Dynamic Gastric Model' (Wickham et al. 2012), the 'Human Gastric Simulator' (Kong \& Singh 2010), and the U-stomacher from our own research group (Luo et al. 2015). 


\section{$\underline{\text { Small intestine }}$}

In order to mimic the digestion in the small intestine in vitro, simulated small intestinal fluid (SSIF) must be prepared with, at least, a neutral $\mathrm{pH}$, relevant enzymes (i.e., proteases for protein hydrolysis and lipase for lipolysis), biological surface-active components (bile salts and phospholipids) and a proper concentration of calcium and other minerals $\left(7.6 \mathrm{mmol} \cdot \mathrm{L}^{-1}\right.$ $\mathrm{K}^{+}$; $123.4 \mathrm{mmol} \cdot \mathrm{L}^{-1} \mathrm{Na}^{+}$; $55.5 \mathrm{mmol} \cdot \mathrm{L}^{-1} \mathrm{Cl}^{-} ; 0.8 \mathrm{mmol} \cdot \mathrm{L}^{-1} \mathrm{H}_{2} \mathrm{PO}_{4}^{-} ; 85 \mathrm{mmol} \cdot \mathrm{L}^{-1} \mathrm{HCO}_{3}{ }^{-}, \mathrm{CO}_{3}{ }^{2-}$ ; $0.33 \mathrm{mmol} \cdot \mathrm{L}^{-1} \mathrm{Mg}^{2+} ; 0.6 \mathrm{mmol} \cdot \mathrm{L}^{-1} \mathrm{Ca}^{2+}$ ) (Minekus et al. 2014). It is important but difficult to get the composition of the SSIF right, since the composition of human digestive juice is complex, dynamic and fluctuating, as previously explained (McConnell et al. 2008), and it can greatly influence the outcome of simulated digestion experiments (Li et al. 2011). Static intestinal models are used mostly, since it is challenging to include the dynamics of intestinal digestion processes in vitro. However, some dynamic models are available, such as the TIM1 system (TNO, The Netherlands).

\section{Colon}

The main role of the colon is to allow bacterial fermentation, hence related in vitro models focus on reproducing the action of the involved microbiota. To investigate ingredient release in the colon using in vitro models, the microbes of the whole gastro-intestinal tract must be taken into account, in addition to all other above-described factors. Example thereof are the TIM-2 system (TNO, The Netherlands) and the Simulator of Human Intestinal Microbial Ecosystem (SHIME), which contains a microbial spectrum (de Boever et al. 2000, Molly et al. 1994). Such models can be used to investigate the degradation pattern of a food matrix, while also taking the microbial ecology into account, instead of just bacteria enumeration. Specifically for the release of probiotic bacteria, it should also be studied whether the cells are still viable when reaching the colon (Cook et al. 2012).

\subsection{SATIETY}

To define two situations of inhibition of food intake, the terms "satiation" and "satiety" are introduced: satiation denotes the inhibitory processes that start during meal intake and cause people to bring an eating episode to an end; satiety denotes the post-meal inhibitory processes that supress the motivation to eat until the next eating episode (Blundell \& Bellisle 2013).

Feelings of hunger and satiety experienced by a person fluctuate over the day, and depend on factors such as earlier meal volume and composition, time since the last meal, energy expenditure and so on. Dietary components are sensed throughout the GI tract, resulting in signalling to regulate hunger, satiety and food intake. Maljaars et al. reviewed the regulation mechanisms of such signalling, which origins in the stomach and small intestine and include 
humoral and neural pathways (Maljaars et al. 2007). The involved mediators include CCK, ghrelin, PYY, GLP-1 and oxyntomodulin (OXM). CCK is a hormone that apart from stimulating gallbladder contraction and pancreatic enzyme secretion, also induces satiety and thereby reduces the food intake (Gibbs \& Smith 1982). It is released by intestinal I-cells primarily in the proximal small intestine, when nutrients are present in the duodenum and jejunum. GLP-1 is a peptide that also inhibits food intake, and acts via receptors in the hypothalamus (Turton et al. 1996). It is mainly released from entero-endocrine L-cells in the distal intestine, as is the case for PYY. Batterham and co-workers reported that infusing PYY at the concentration that is normal after a meal $\left(0.8 \mathrm{pmol} \cdot \mathrm{kg}^{-1} \cdot \mathrm{min}^{-1}\right.$ for $\left.90 \mathrm{~min}\right)$ reduced the energy intake in the $24 \mathrm{~h}$ after the infusion by $33 \%$, compared to a control infusion with saline (Batterham et al. 2002). After a meal, the plasma concentrations of PYY are dependent on the caloric meal composition (Pedersen-Bjergaard et al. 1996). OXM is co-secreted with PYY and GLP-1 by L-cells in the distal intestine and colon, and reduces gastrointestinal motility, induces satiety and decreases food intake (Maljaars et al. 2008a). In contrast to these satiety-inducing gut peptides, the hormone ghrelin stimulates appetite and food intake (Inui et al. 2004). Ghrelin is mainly secreted in the stomach by special gastric cells, also called $\mathrm{X} / \mathrm{A}$ like cells.

\subsubsection{Satiety signals induced by undigested nutrients in the small intestine}

Transposing a small segment of distal ileum to the jejunum lowered the food consumption of rats, and caused more weight loss than in the control rats (Strader et al. 2005). This was associated with a higher release of GLP-1 and PYY in the test rats, and not due to malabsorption, indicating that the distal part of the small intestine is more responsive to nutrients than the more proximal parts, with respect to induction of satiety signals. The presence of unabsorbed nutrients in the ileum is thought to be able to induce satiety, via the so-called ileal brake and thereby reduce food intake (Maljaars et al. 2008a). Several human intubation studies have been done to investigate this mechanism (Maljaars et al. 2008b, 2009, 2011; van Avesaat et al. 2015). In 2008, Maljaars et al. investigated the effect of infusion of fat (low or high dose, respectively $3 \mathrm{~g}$ and $9 \mathrm{~g}$ emulsified safflower oil) into the ileum on satiety as compared to a control treatment (oral fat and a saline infusion). The authors found the PYY secretion to be only increased with high fat perfusion in the ileum and not with the lower dose, and CCK was secreted dose-dependent correlating with the levels of satiety (Maljaars et al. 2008b). After that, Maljaars and co-workers investigated the effect of ileal perfusion of different oil types -including shea oil (mainly stearic acid, C18:0), canola oil (mainly oleic acid, C18:1), and safflower oil (mainly linoleic acid, C18:2)- on satiety and food intake, as compared to a saline infusion (Maljaars et al. 2009). No direct effect on food intake was observed and PYY was not affected; however, the authors did find significantly increased satiety feelings, reduced hunger and increased CCK secretion by C18:2 and C18:1 
fatty acids (FAs) compared to the control and compared to shea oil (Maljaars et al. 2009). Van Avesaat et al. (van Avesaat et al. 2015) recently reported that protein and carbohydrate infusion in the ileum can also reduce the energy intake, compared to the control treatment (saline infusion). The CCK and PYY secretion was increased for all types of macronutrients, the gastric emptying rate and intestinal transit time seemed to be delayed, but GLP-1 secretion was not affected (van Avesaat et al. 2015). Thus, the ileal brake can also be activated by other macronutrients than lipids.

The intestinal area exposed to fat may also influence the feelings of satiety. Maljaars et al. investigated this by infusing $2 \mathrm{~g}$ of fat each in the duodenum, jejunum and ileum, or $6 \mathrm{~g}$ of fat into the ileum, and measuring the effect on satiety compared to a control treatment (6 g fat in liquid meal and saline infusion) (Maljaars et al. 2011). None of the treatments resulted in differences in the amount of secreted PYY, CCK nor GLP-1 compared to the control treatment, but the feelings of hunger were reduced by infusion of $6 \mathrm{~g}$ fat simultaneously in the three segments ( $2 \mathrm{~g}$ per segment) or in the ileum. The ileal infusion also reduced food intake compared to oral administration of a liquid meal containing $6 \mathrm{~g}$ of fat, so the duration and site of exposure influences hunger and food intake (Maljaars et al. 2011). Besides, also duodenal infusion of glucose (Lavin et al. 1998, Pilichiewicz et al. 2007) or protein (Geraedts et al. 2011, Ryan et al. 2012) decreased energy intake when compared to placebo and increased plasma levels of satiety inducing hormones GLP-1 and, for protein infusion, CCK.

\subsection{LIPOLYSIS}

As mentioned in the previous sections, fats and oil play an important role in our metabolism, and may influence satiety to a large extent. In this section we focus on the process of lipolysis, and we will discuss in section 2.6 how lipolysis can be prevented, or at least delayed.

In the human GI tract, the digestion of lipophilic materials starts in the stomach, but mainly takes place in the small intestine, where the digestion products are also absorbed. Dietary oils and fats are mainly triacylglycerols (TAGs), which are digested by region-specific triacylglycerol hydrolases (lipases) that cut the FAs esterified to the sn- 1 and sn-3 positions on the glycerol backbone. Lipases are known to act on an interface, where the water-soluble lipase and the lipid substrate meet.

In the human digestive system, the two main types of lipolytic enzymes secreted are human gastric lipase (HGL) in the stomach, and human pancreatic lipase (HPL) in the small intestine. Both have a size of about $50 \mathrm{kDa}$. Most digestion studies use only pancreatic lipase as a lipolytic enzyme, even though gastric lipase, pancreatic carboxyl-ester hydrolase and pancreatic lipase-related protein 2 have also been mentioned to have an important role in digestion of acylglycerols (Bakala N’Goma et al. 2012). HPL needs a co-lipase (a protein 
cofactor, 5 times smaller than the lipase itself) that enables hydrolysis of TAGs, via avoiding the effect of bile salts that remove HPL from the interface (Bakala N'Goma et al. 2012). After 3 h digestion, a 4-time higher amount of HPL is secreted than HGL, but HGL is active for a longer time (in both the stomach and small intestine), so over the whole digestion trajectory HPL hydrolysis contributes only three times more than HGL to the overall conversion of TAGs (Carriere et al. 1993).

The amount of interfacial area between lipids and the surrounding aqueous medium, as well as the interfacial structure, also affect the rate and extent of lipolysis. The amount of interfacial area is defined by the size of the lipid droplets, and increases when the droplet size decreases, due to a higher surface to volume ratio. Depending on the ratio between the amount of enzyme and the available surface area, either the amount of enzyme, or the available surface area is rate limiting. For example, Li et al. (2011) found that the initial lipolysis rate increased with increasing lipase concentration up to $4.8 \mathrm{mg} \cdot \mathrm{mL}^{-1}$, and this indicates that the amount of lipase is rate limiting ( $\mathrm{Li}$ et al. 2011). Surface saturation has been reported, but outside the area of investigation that we report on here, e.g., in biotechnology it was found that the reactor conversion rate increase linearly upon addition of enzyme (at low concentration) but did not increase anymore upon further addition of enzyme.

Decreasing the droplet size of Tween 80-stabilized emulsions from $2.3 \mu \mathrm{m}$ to $0.2 \mu \mathrm{m}$ increased the initial rate of in vitro digestion by a 4-fold factor; when just looking at the size (and total surface) ratio this should have been a factor of 11, but probably the droplet size distribution played a role here, or the available amount of lipase was not sufficient to cover all the interface related to the small droplets. Also the amount of FFAs released after $2 \mathrm{~h}$ increased slightly from about 57 to $67 \%$, as well as the in vivo bioavailability of coenzyme Q10 increased (Cho et al. 2014). Comparable results for in vitro digestion were obtained with $\beta$-lactoglobulin-stabilized emulsion droplets: the lipolysis in the first 3 min decreased from about 54\% FFA release for small droplets (178 nm) to $48 \%$ for 1.4 times bigger droplets and to only 19\% FFA release for 4.3-time bigger droplets (758 nm) (McClements \& Li 2010b). When testing even smaller droplets, contradictory results were obtained; $\beta$-lactoglobulinstabilized emulsions with a droplet size of $60 \mathrm{~nm}$ were digested slower compared to $200 \mathrm{~nm}$ droplets (McClements \& Li 2010b). The authors have related this finding to the interfacial structure that may be different due to another preparation method. This explanation is in line with the findings in a recent study by Garcia and co-workers, on native and homogenized milk fat globules: When native milk fat globules were subjected to digestion, the lipolysis rate increased when the average globule size decreased (from $6.6 \mu \mathrm{m}$ to $1.7 \mu \mathrm{m}$ ), due to more available interfacial area. When the globule size was further reduced by high pressure homogenization (down to $0.3 \mu \mathrm{m}$ ), no increase in the lipolysis rate was observed. This may be related to the composition and physical structure of the interfacial layer surrounding the 
globules (Garcia et al. 2014); at the same time, it could also indicate that there was not sufficient lipase to cover the available area. These examples show that different effects of the droplet size have been observed, and they could be very well related to a change in limitation (so either amount of enzyme, or amount of surface area, as previously stated). It should also be pointed out that mostly the initial lipid droplet size is measured, and effects are related to it, but the actual droplet size often varies throughout the digestive tract, which makes it a complex factor to study.

Besides, it can be expected that the total amount of interfacial area is not the only factor controlling lipolysis, but the physical organization of the interface and the lipid composition also matter. The effect of different interfacial structures on lipolysis reduction is discussed in section 6 on 'delayed lipolysis to induce satiety'. The lipolysis rate is determined by the composition of the lipid, since the affinity of the enzyme depends on the substrate composition, and the same would be true for the solubility of the formed digestion products (Marze et al. 2014). The degree of saturation of the fatty acids (FAs) corresponds to the solid fat content (SFC); at high degree of saturation, the SFC of a lipid will be higher at body temperature (for the same alkyl chain length). The molecules associate in a crystalline lattice, which is more dense, less mobile and more ordered than liquid oil. This could lead to a lower accessibility of TAG molecules to lipase, which may explain the lower digestibility (Bonnaire et al. 2008). In addition, the position of the fatty acids on the glycerol backbone influences the lipolysis as lipase has a region-specific affinity (McClements et al. 2009). Besides, the length of the FAs affects the rate and extent of lipolysis, the latter being higher for shorter FAs (Marze et al. 2014). This was illustrated by Giang et al. (2015) who monitored intestinal in vitro digestion of TAGs with medium chain FAs (MCT), against TAGs with long chain FAs (LCT), and found that MCTs were digested in 15 min, whereas LCT remained undigested after $5 \mathrm{~h}$. The latter effect is caused by coalescence of the oil resulting in a reduced interfacial area, and consequently less lipase activity.

In order to ensure optimal lipolysis in the GI tract, several natural mechanisms are involved in increasing the interfacial area, and preventing inhibition due to reaction products. For one TAG molecule, lipolysis results in two free FAs and a monoacylglycerol (MAG) with a fatty acid at the sn-2 position. The MAG itself is surface active, but also highly surface-active components are secreted into the duodenum, such as bile salts and phospholipids. These components stabilize newly created oil-water interface to facilitate lipase action (Maldonado-Valderrama et al. 2011). Bile salts are also able to displace certain molecules from the oil-water interface, via competitive adsorption. This phenomenon has been referred to as orogenic displacement, and has been found to start with adsorption of the competing species at defects in the interfacial network (Figure 2.3a). After this, the domains of the competing species grow from this nucleation site (Figure 2.3b), compress the initially 
adsorbed material, and thereby force it to desorb (Mackie et al. 1999), which lines the surface up for lipolysis (Figure 2.3c) (Maldonado-Valderrama et al. 2011).

The formed MAGs are surface-active enough to remove lipase from the interface at a certain surface coverage, and are part of a self-regulatory mechanism (Reis et al. 2008). On the other hand, bile salts are able to remove MAG's from the interface and thereby promote further lipolysis (Figure 2.3d). This is done through mixed MAG-bile salt micelles that transport the digestion products through the mucus layer, towards the epithelial cells (MaldonadoValderrama et al. 2011). Calcium plays a multiple role in lipolysis, as it is a co-factor in the activation of HPL, and additionally, it may bind long-chain FAs, precipitate them as soaps, and thereby prevent inhibition of the enzyme at the interface by the digestion products $(\mathrm{Hu}$ et al. 2010a). Besides, as mentioned previously, calcium may affect droplet flocculation, and also have an effect on biopolymer aggregation and/or bile salt precipitation.

Other factors, such as the feeding status and food matrix composition, can also impact lipolysis (Christophersen et al. 2014). Food matrix components - especially dietary fibres can directly interact with lipase, bind bile salts, form a protective membrane around lipid droplets, or enhance the viscosity and thereby change the behaviour in the GI tract, as has been reviewed by McClements (McClements et al. 2009), and therefore considered outside the scope of this paper.

The release of lipophilic drugs/functional ingredients loaded into lipid-based carriers is often lipolysis rate-dependent (Bakala N'Goma et al. 2012, Garti et al. 2012). These latter authors showed that drug release from a reversed hexagonal lipid increased from less than $5 \%$ after

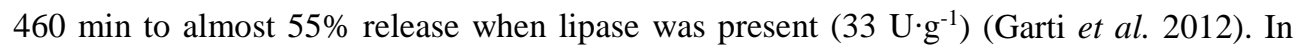
another example, the in vivo bioavailability of a lipophilic nutraceutical in rats was higher when dissolved in digestible corn oil compared to indigestible mineral oil (and higher when the droplets were smaller) (Cho et al. 2014). However, Ahmed et al. (2012) showed the bioaccessibility of curcumin to decrease in the order medium $>$ long $>$ short chain triacylglycerols, while the initial digestion rate decreased in the order SCT > MCT > LCT, and the final digestion decreased in the order MCT > SCT > LCT. This indicates that bioaccessibility of components is much more complex than just related to the rate of lipolysis of the carrier material. 


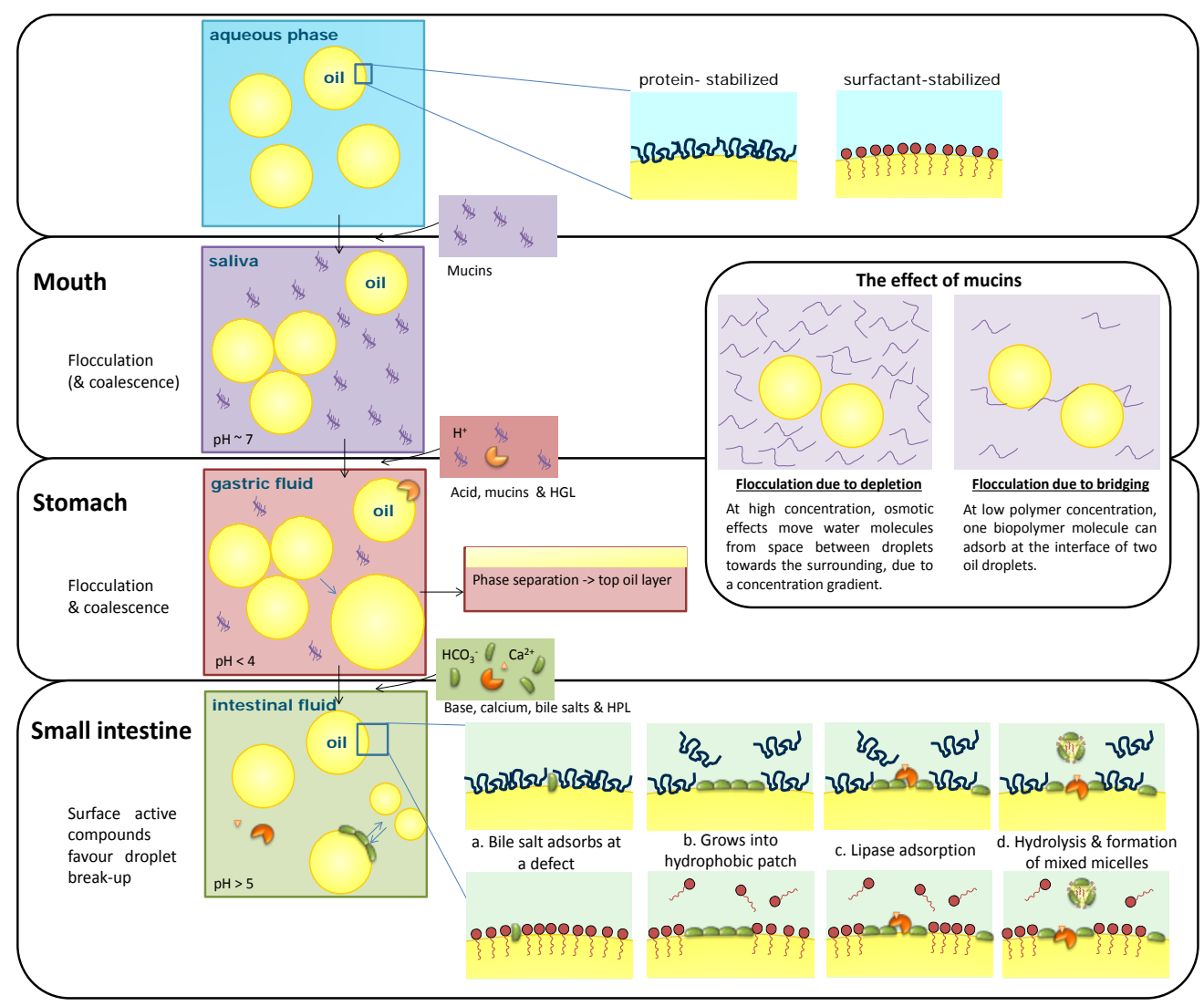

Figure 2.3. Schematic representation of the effect of the components of the GI tract on an emulsion and its interface.

\subsection{ENCAPSULATION}

Encapsulation technology is in use to carry a functional ingredient, for instance in food and pharmaceutical applications, which needs to be protected from the influences of the GI tract, to a location at which the content should be released. Various large-scale options are available, such as the filled hydrogel particles (Tan et al. 2009) or organogel particles (Duffy et al. 2009), while other capsules contain a protective shell/interface that controls the release. This shell may be made through e.g., layer-by-layer adsorption or spray coating. Recent trends in encapsulation techniques and materials that are suitable for micro-encapsulation of lipophilic materials that ultimately may be used to reduce induce satiety are summarized in section 2.6. This is followed by examples where controlled digestion or release was achieved via responsiveness of the systems to external stimuli as present in the GI tract. Finally, the role of dynamic partitioning of molecules in encapsulation system is discussed. 


\subsubsection{Micro-encapsulation techniques}

The main methods to create encapsulates include layer-by-layer adsorption, spray-drying, fluidized bed coating, extrusion, coacervation, and solvent change or removal. In the food industry, hydrophobic ingredients are often encapsulated based on an oil-in-water emulsion system, of which the interfacial layer is engineered in order to control the release and/or digestion. The production method defines the size of the capsules, which can be on micronscale (several hundreds of nanometers to some hundreds of micrometers) that are called microcapsules (Neubauer et al. 2014) or even smaller as is the case in nanodelivery systems $(<200 \mathrm{~nm})$ as classified in the review of Borel \& Sabliov (2014). The size of the capsules determines the volume of encapsulated material, and the interfacial area, which increases reciprocally with size. Both aspects are important for digestion and/or release-related aspects. Besides, capsule shell structure (thickness, porosity, homogeneity) will be of influence on the final functionality of the capsule.

\section{Emulsion-based systems}

Encapsulates are frequently produced starting from emulsions (Day et al. 2014, Li et al. 2010, Mantovani et al. 2013, McClements et al. 2007), and if well-defined capsules are required, the emulsification method should be chosen with care. For high throughput processes, traditional devices, such as high pressure homogenisers, colloid mills, etc. are used, but these are not known for their control of droplet size. In that respect, the microstructured devices that are becoming of age nowadays, may be interesting alternatives, although it should be mentioned that throughput may still be an issue (Schroën et al. 2015, Yuan \& Williams 2014).

As mentioned in the previous section, various options exist to stabilised the oil-water interface, and we already discussed low molecular weight surfactants and amphiphilic biopolymers, solid (lipid) particles, and filled hydrogel particles, (McClements \& Li 2010b). Now we take interfacial structure one step further, and relate that to methods that can be used to make capsules.

\section{Layer-by-layer}

To alter the structure and properties of the surface of an emulsion droplet, additional layers can be added on top of the primary emulsifier. The use of layer-by-layer approaches has even been postulated by Neubauer et al. (2014) as one of the best ways to control the capsule's mechanical performance, compared to other strategies. In literature it has been stated that multilayered systems could be useful to obtain improved stability against environmental stresses ( $\mathrm{pH}$, salt, thermal, lipid oxidation, dehydration) and controlled release/triggered release (Guzey \& McClements 2006). 
The physical principles governing multilayer formation is extensively reviewed by Schönhoff (2003). The layers can be added by adsorption based on electrostatic attraction of oppositely charged molecules, and this technique is used to build multilayered capsules (Antipina \& Sukhorukov 2011, Deligöz \& Tieke 2014, Hu et al. 2010b, Luo et al. 2013, Rossier-Miranda et al. 2012, Sakr \& Borchard 2013, Zeeb et al. 2014). As was already clear from the emulsion section, environmental factors and choice of components greatly decides on the result that will be obtained. We will not go into details here, but focus on the charge differences between components that are needed to form consecutive layers, and are the basis for the formation of these capsules.

Depending on the dissociation constant of a component it will carry more or less charge as function of $\mathrm{pH}$. In order to adsorb an additional layer onto an emulsion droplet, the charge of the next component needs to be opposite to the surface charge of the droplet. The distance over which the droplet charge is notable is called the Debye length $\kappa^{-1}$ and is expressed as presented in Equation 2.1 (Russel et al. 1989).

$$
\kappa^{-1}=\sqrt{\left(\varepsilon_{\mathrm{r}} \varepsilon_{0} \mathrm{k}_{\mathrm{B}} \mathrm{T}\right) /\left(2 \mathrm{~N}_{\mathrm{A}} \mathrm{e}^{2} \mathrm{I}\right)}
$$

Equation 2.1

where $\varepsilon_{0}$ is the permittivity of free space, $\varepsilon_{\mathrm{r}}$ is the dielectric constant, $k_{\mathrm{B}}$ is the Boltzmann constant, $T$ is the absolute temperature, $N_{A}$ is the Avogadro number, $e$ is the elementary charge and $I$ is the ionic strength of the electrolyte.

From this equation it can be seen that the Debye length is larger when the ionic strength is lower, and this influences how densely molecules can arrange in and between layers. The permeability of a capsule with a fixed composition, can be tuned by changing the preparation parameters, and thereby the porosity of the shell (Klitzing 2006). It is good to mention that the ionic strength and $\mathrm{pH}$ also affect emulsion stability against flocculation, but we consider that outside the scope of the current review.

A nice example of mechanically very strong multilayered capsules can be found in the work of Rossier-Miranda and co-workers (2010), who also used the charge interactions between components, but did not just use molecules but also protein fibrils to fortify the shells. As a result, these capsules that were produced at low $\mathrm{pH}$, were found to be very stable at that $\mathrm{pH}$, while they disintegrate at high $\mathrm{pH}$, because of the loss of the charge interactions. Also an effect of the number of layers was reported; at 8 and more layers, the complete capsule was neatly covered with a coat, while at lower number of layers, holes were detected that also lead to premature disintegration of the capsules, compared to those that had 8 or more layers, and that were shown to be stable at $\mathrm{pH} 2$ and $\mathrm{pH} 7$ for more than $4 \mathrm{~h}$ (Rossier-Miranda et al. 2010). 


\section{Fluidized-bed coating}

Alternatively, a shell can be applied by spray-coating in a fluidized bed, and in this case particles need to be used in the range of 5-5,000 $\mu \mathrm{m}$ (Zuidam \& Shimoni 2010). To get a gradual and equally distributed coverage, the particles are suspended in an air flow at an appropriate temperature and coated in the spray zone by atomized coating material, which can be the material as such or dissolved in an appropriate solvent that is subsequently removed. The rate of drying must be fast enough to prevent agglomeration of the capsules, and slow enough to allow for homogenous coating formation (Santivarangkna et al. 2007). In some cases, an additional drying step needs to be carried out in the fluidized bed (Situ et al. 2014).

The preferred thickness of shell depends on the application, and defines the amount of coating material to be sprayed per unit of interfacial area. The relative amount of coating is typically between 5 and 50\%; most used materials are cellulose derivatives, dextrins, proteins, gums and/or starch derivatives (Zuidam \& Shimoni 2010). Multilayered capsules can also be produced, by repeated exposure to different materials (Vitaglione et al. 2012).

\section{Extrusion}

The typical capsule size that can be achieved by classic extrusion is $300-5,000 \mu \mathrm{m}$, and this is also the case for particles; both emulsions and particles are rather polydisperse in size (Zuidam \& Shimoni 2010). For this, the core material is added to a hot biopolymer solution before extrusion. Upon exiting the machine, the product enters a hardening bath, and a glassy coating is produced, resulting in a good barrier for volatile components such as flavours (Madene et al. 2006). Since the extruder is shear and temperature intensive, the components and process conditions need to be chosen with care (Emin et al. 2012).

In syringe extrusion, a viscous solution is extruded drop-wise into a hardening solution, without requirement of high temperatures. It is typically used to produce alginate (-chitosan) beads with active cores, by extruding them into a calcium chloride solution where the droplets gel (Tan et al. 2009, Yan et al. 2014). Alternatively, a spinning disk can be used, and also actuated nozzles have been reported for the large scale production of biocatalyst beads that generally are in the same size range as stated for extrusion, but uniform in size.

Also premix membrane emulsification is sometimes termed extrusion, since it involves passing an emulsion through a membrane, which leads to reduction of the droplet size, to values that are 2-5 times the size of the pore applied. Mostly the particles are in the 0.2 to 2 $\mu \mathrm{m}$ range, and not completely monodisperse, but the size distribution is much better as found in regular extrusion. Besides for emulsion formation, the same technique can also be used to make both solid and hollow particle (the latter can e.g., be used as ultrasound contrast agents (Sawalha et al. 2008, 2009, 2011). 


\section{Methods using phase separation}

The quality of a solvent determines whether a component stays in solution or is more likely to form another phase. First, the solvent, or co-solvent can be removed, therewith inducing a phase transition, and that phase transition may take place on a template emulsion droplet as nicely illustrated by Sawalha and co-workers for ultra-sound contrast agents (Sawalha et al. 2008, 2009, 2011). They made tetradecane filled polylactic acid (PLA) microcapsules, starting from PLA dissolved in dichloromethane (DCM) and with tetradecane added, which was brought into contact with a water phase and premix-emulsified. The DCM dissolves in water, leading to precipitation of the PLA on the tetradecane droplets. Also polycaprolactone or polystyrene (Shahidan et al. 2013) and poly(lactic-coglycolic acid)-based microparticle loaded with a drug (Krishnamachari et al. 2007, Wu et al. 2012) have been reported in literature to be produced in this way. Alternatively, an anti-solvent can be added which may also lead to solute precipitation (Joye \& McClements 2013).

Also emulsion coacervation can be termed a method that uses phase separation. In this case, liquid-liquid phase separation results in a polymer-rich and a polymer-lean phase. When starting from and emulsion, the polymer-rich phase deposits at the emulsion interface, due to interaction between both biopolymer solutions at the oil in water interface (Butstraen \& Salaün 2014). The interface can be firmed by a cooling step below the gelling temperature of the polymer phase, or by cross-linking (Xiao et al. 2014), after which the coacervates can be separated, and dried if needed.

At least two polymers with opposite charges are needed for so-called complex coacervate formation. This can be modulated through $\mathrm{pH}$, temperature, or ionic strength. Gelatin and gum arabic are the most used polymers to form the shell, but a wide range of materials can be used: proteins extracted from animal-derived products (gelatin, whey proteins, silk fibroin) and from vegetables (soy proteins, pea proteins), and polysaccharides such as gum arabic, pectin, agar, alginate, carrageenan and carboxymethyl cellulose (Xiao et al. 2014). Chitosan also gets more and more attention, as it is one of the few polycationic biopolymers available and is suitable for the production of coacervated capsules for controlled release (Butstraen \& Salaün 2014, Yang et al. 2014b).

\section{Spray drying of emulsions}

Spray drying is widely used to improved shelf life, and stability of ingredients, e.g., in capsules that contain flavours, lipids or carotenoids (Gharsallaoui et al. 2007). The feed stream is atomized into hot air to rapidly evaporate the solvent, and in that respect could also be termed a method that uses phase transition. The feed contains all ingredients for example, canola oil emulsions (50\% oil on dry basis, O/W) were made with different structuring materials in the aqueous phase (WPI alone, or protein (NaCas, WPI, HWP, SPI) in 
combination with one or more carbohydrates (processed Hylon VII, oligofructose, dried glucose syrup or pectin)) and all these mixes were successfully spray dried into microencapsulated oil powders (Augustin et al. 2014). Turchiuli et al., succeeded to encapsulate tocopherol by spray drying an initial emulsion containing different carrier materials (combinations of maltodextrin, acacia gum and agave inulin) (Turchiuli et al. 2014). They used $40 \% \mathrm{w} / \mathrm{w}$ dry mass of which $8 \%$ olive oil and $2 \%$ tocopherol, and found the initial emulsion structure to be maintained after re-dispersing in water.

A hydrophobic functional ingredient would be added to the oil phase of the emulsion, while a hydrophilic component mostly adheres to the shell material, or is incorporated into a particle that does not contain oil. The feed emulsion should not be too viscous in order to allow spraying. Typical matrix materials are gum arabic, milk proteins, soy proteins, modified starch and maltodextrins (Augustin \& Hemar 2009); point of attention is that the shell should not become glassy when encapsulation of low molecular weight components is targeted (Augustin \& Hemar 2009). Spray drying will result in a high quality product when the heat and mass transfer processes are well controlled, which in general is very well feasible. Alternatively spray chilling can be used that operates at low outlet temperature.

\subsubsection{Encapsulation materials}

A wide range of materials has been used to construct the shell of encapsulation systems, including lipid-, protein- and carbohydrate- based ingredients, and frequently combinations thereof, and here we present them based on the following categories: polymers (including proteins), particles and low molecular weight components. The materials at an oil-water interface are visualized in Figure 2.4; digestibility, and bioaccessibility all depend on the properties of the formed layer (Cilla et al. 2012, Marze 2013). For example, the digestion of protein molecules will be different compared to aggregated proteins (Barbé et al. 2013), but also the subsequent presence of proteins and polysaccharides in multilayered structures is expected to influence the action of enzymes involved in digestion.

\section{Polymer-based materials}

Both biopolymers and synthetic polymers are discussed below. Commonly, more than one polymer is used, which can be applied as a blend or subsequently; in both cases, aiming at enhanced functionality. 


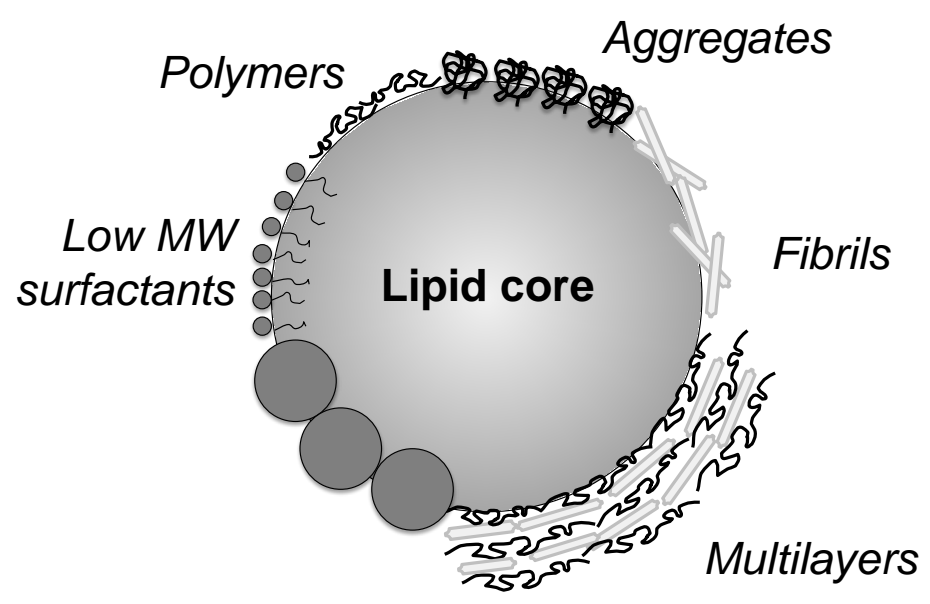

Figure 2.4. Schematic representation of materials at an oil-water interface; drawing is not to scale.

\section{Biopolymers}

The two main biopolymers that occur in nature are proteins (amino acids as building blocks) and polysaccharides (sugar units). In drug micro-encapsulation, the most used biopolymers are gelatin (a protein), chitosan, alginate and cellulose (polysaccharides) (Lam \& Gambari 2014). Gelatin is derived from collagen, which forms a viscous liquid above $30-40^{\circ} \mathrm{C}$ and a gel upon cooling. Chitosan is a linear cationic polysaccharide obtained from crustacean shells, which has low digestibility and weak short-lasting mucoadhesion (Grabovac et al. 2005). Alginate is a cell wall component of brown algae, and often used for its gelling behaviour with calcium ions (Paques et al. 2014). Cellulose is an insoluble linear polymer obtained from the primary cell wall of green plants, and some algae and bacteria. Chitosan, alginate and cellulose have different charges, alginate being anionic, cellulose non-ionic and chitosan cationic.

In the food industry, other components maybe be used for instance, dairy proteins (caseins, whey proteins), egg, and plant proteins (e.g., soy, pea), starch, dextran, agar, galactomannans, pectin, xanthan, carrageenan, gum arabic, gellan (McClements et al. 2007). In general, the costs allowed for encapsulation are much less in food as compared to pharma, therefore in general, less expensive ingredients are used, which have a lower purity. Still chemical modification may be used to enhance the functionality of any of the previously mentioned ingredients (Gibbs et al. 1999). The length is adjusted, or side groups can be added, removed or modified seemingly at will, mostly leading to improved solubility, interfacial activity, or reduced viscosity, which is essential in the production of capsules. Modification can also be used to affect the interaction within in the GI tract, such as increasing the mucoadhesion by thiolation, which is favourable for the release of specific drugs (Grabovac et al. 2005). 
However, it is difficult to make ingredients that are satisfactory, both from a production and release point of view. Another disadvantage of such ingredients is that they are not always allowed to use in food, for example the use of covalently modified starch with octenyl succinic anhydride (OSA) is approved as food additive (E1450) up to a 3\% degree of modification based on the dry weight of starch (Rayner et al. 2012).

Besides protein molecules, also protein fibrils have attracted attention as building blocks for capsules. These can be made by heating an acidic protein or peptide solution under some stirring, which leads to both hydrolysis and fibril assembly at the same time, albeit at a different rate. The fibril length depends on reaction-time and shear rate (Akkermans et al. 2008), and they have been used to reinforce microcapsules (Kroes-Nijboer et al. 2012, Rossier-Miranda et al. 2010). Recently, $\beta$-LG fibrils were found not only to provide a more elastic interface compared to native $\beta$-LG, but also a better oxidative stability to encapsulated fish oil core (Serfert et al. 2014). At the same time it should be mentioned that some concerns exist regarding the potential toxicity of protein fibrils because these nanostructures might relate to protein misfolding diseases (e.g., CJD, Alzheimer's, Parkinson's and Huntington's diseases) (Raynes et al. 2014).

As mentioned previously, also supra-molecular structures such as coacervates can be used to stabilise capsules. Protein-polysaccharide complexes may be formed under conditions that favour both hydrophobic and electrostatic interactions. Four $\mathrm{pH}$-regions can be distinguished (Li et al. 2013); above a certain $\mathrm{pH}$, the polymers are dissociated. Upon acidification, soluble complexes will form till a certain $\mathrm{pH}$ is reached at which they start to form insoluble complexes due to aggregation. At an even lower $\mathrm{pH}$, the complexes disassociate again into separate polymers. These regions also depend on the protein/polysaccharide ratio as shown by Li et al. (2013).

Protein-polysaccharide conjugates also consist of proteins and polysaccharides, but these are covalently coupled, through heating of a dry mixture of proteins and polysaccharides, leading to Maillard reaction products (Xu et al. 2012). Although conjugated WPI and pectin are able to improve the physical stability of emulsions compared to unconjugated WPI-pectin mixture (Xu et al. 2012), lipid digestion in these emulsions was not affected (Xu et al. 2014).

\section{Synthetic (co-)polymers}

A limited amount of synthetic polymers can be applied in pharma (and to some extent in food). Examples are acrylic polymers (Eudragit) (Ibekwe et al. 2006, Krishnamachari et al. 2007) and polylactide (Sawalha et al. 2009). Safety of components can be found on the websites of the European Food Safety Authorisation (EFSA 2014), for example Eudragit (EFSA 2010), and in the USA on the website of the Food and Drug Administration (FDA 2014). 


\section{Colloidal particles}

Pickering emulsions are known to be very stable in solution due to the presence of colloidal particles in their interface (Pickering 1907). These particles may be added through the continuous phase, or the dispersed phase; and need to have dual affinity for oil and water, which gives them the potential to practically irreversibly adsorb at the interface and thereby physically stabilize the droplets. The adsorption/desorption energy of a particle is generally estimated by the relationship in Equation 2.2 (Binks 2002, Leal-Calderon et al. 2007).

$$
E=\gamma_{\text {int }} \pi R^{2}(1-|\cos \theta|)^{2}
$$

with $\gamma_{\text {int }}$ being the interfacial tension between oil and water phases, $R$ the particle radius and $\theta$ the contact angle between the particle tangent at contact and the interface.

From this equation it can be seen that a contact angle of $90^{\circ}$ (resulting in a $\cos \theta$ of zero) gives the highest desorption energy $E$, and the most irreversible adsorption. The amount of energy involved in binding very small particles is much larger as that generated in Brownian motion, therewith making desorption practically impossible, while the Brownian motion of low molecular weight surfactants is still such that they may leave the interface.

The use of particles for food emulsion stabilisation has recently been reviewed by our group (Berton-carabin \& Schroën 2014). The particles used for Pickering food applications are mostly protein- or carbohydrate-based, such as chitin nanocrystals (Tzoumaki et al. 2013), colloidal lactoferrin particles (Meshulam \& Lesmes 2013) and coacervated WPI-pectin particles (Salminen \& Weiss 2014). Also colloidal solid lipid particles and surfactant-based crystals were reported (Gupta \& Rousseau 2012, Rousseau 2013), which can be prepared by cooling from the liquid state. Alternatively, lipid ingredients can be dissolved in a solvent, such as ethanol or acetone, and dispersed into an aqueous phase containing emulsifier, after which phase separation sets in; e.g., phytosterol particles were produced in this way (Liu \& Tang 2014). Recently, micron-sized microorganisms were also described as food-grade Pickering stabilisers, in combination with maltodextrin-gelatin systems (Firoozmand \& Rousseau 2014). Besides also silica particles were used in a multilayered shell (RossierMiranda et al. 2012) or in a W/O/W emulsion (Chen et al. 2014).

\section{Low molecular weight components}

Typical components that fall in this category have a molar weight between about 250 and $1200 \mathrm{~g} \cdot \mathrm{mol}^{-1}$ (Berton-carabin \& Schroën 2014), with a polar head and an apolar fatty acid tail. Some surfactants are natural polar lipids (e.g., lecithin), but most components are produced by synthetic esterification of fatty acids (10-20 C atoms long) and polar molecules. 
The polar headgroup may be just one or more glycerol units, or glycerol esterified with acid as is the case in CITREM, DATEM, and LACTEM, or with a sugar group, as is the case in sucrose esters, galactolipids; or the headgroup may be sorbitan (Span) or sorbitan with polyoxyethylene groups (Tweens). The length and degree of saturation of the FAs mostly determines the melting point of the surfactant.

Depending on their so-called hydrophilic/lipophilic balance (HLB), surfactants are more likely to form oil in water (high HLB) or water in oil emulsions (low HLB); this is known as Bancroft's rule. To make a double emulsion (W/O/W or $\mathrm{O} / \mathrm{W} / \mathrm{O})$, both are needed (Park et al. 2014). Besides the charge of the head group is relevant. Anionic surfactants have a negatively charge (e.g., SSL, CITREM, DATEM, and LACTEM) and are more commonly encountered in foods than cationic surfactants. An example of a zwitterionic surfactant is lecithin, and non-ionic surfactants are e.g., polysorbates and sorbitan esters. Depending on their charge, surfactants will be more or less closely packed, and may result in repulsion between emulsion droplets. The overall geometry of the surfactant determines their preferential way of assembling in supra-molecular structures. Cone-shaped surfactants will form micelles or reversed micelles, cylindrical-shaped surfactants are likely self-assembled into lamellar layers and the "truncated cone" surfactants will form vesicles. An example of this is the so-called liposome, a double layer of phospholipids surrounding a water phase that is typically in the range of $0.1-1 \mu \mathrm{m}$. Also nano-vesicles have been reported, consisting of hydrophobin, an amphipathic protein with high cysteine content. These vehicles (235 nm) effectively encapsulated vitamin D without loss after 3 weeks of storage (Israeli-Lev \& Livney 2014).

Nonpolar lipids. High melting point lipids (HML) can be sprayed in liquid state onto capsules and when cooled they crystallize and form a solid coating that provides barrier properties. For example, curcumin encapsulated in a hydrogenated oil coating was found to improve bioavailability in bread (Vitaglione et al. 2012), and $\mathrm{NaCl}$ encapsulated in a coating of stearic/palmitic acid blend, candelilla wax or carnauba wax, was found to reduce the Maillard reaction (Fiore et al. 2012). HML is also used in drug encapsulation systems such as lipospheres that have a solid lipid core and an embedded phospholipid shell (Elgart et al. 2012).

\subsubsection{Responsiveness}

Apart from the design of the microcapsule and its stability during preparation and storage, obviously also an appropriate trigger needs to be present that allows release at the required position. These triggers can be biological, chemical or physical. The use of biological stimuli, 
like enzymes (Park et al. 2014) and receptors, is less common than chemical and physical triggers as those are more difficult to control and to investigate.

The most used trigger for disrupting edible microcapsules is $\mathrm{pH}$ (Abbaspourrad et al. 2013, Gun \& Routh 2013, Ibekwe et al. 2006, 2008; Laouini et al. 2013, Patel et al. 2013, RossierMiranda et al. 2010); other chemical triggers relate to ionic strength, solvent removal, and electrochemical effects.

Physical stimuli that can be used to induce release of encapsulated molecules include light, electric or magnetic signals, ultrasound (Wrenn et al. 2012), mechanical forces, or temperature (Choi et al. 2010). For medical applications, microcapsules have been made light sensitive through the use of specific polymers, functional dyes and metal nanoparticles (Bédard et al. 2010), although it should be mentioned that it is not expected to be of great use for food applications.

The design of capsules for controlled digestion is more challenging than inducing responsiveness to a single stimulus due to the complexity of the GI tract. In that respect, multilayered microcapsules may need repeated triggers prior to disintegration, and that may be beneficial from a residence time point of view, which may be extended because of this. This type of research is still very early on in its development, but some examples can be found (Antipina \& Sukhorukov 2011). Besides, interindividual variability has to be considered, and this may require personalised production of capsules. In order to achieve this, reversed engineering may be used to lead to better insights in delivery, unlike the current approach that seems to be more formulation driven (Cerqueira et al. 2013).

\subsubsection{Partitioning}

One of the factors that has hardly been touched in literature is partitioning of (active) components. Encapsulation systems for delayed lipolysis, such as $\mathrm{O} / \mathrm{W}$ emulsions, contain three regions: a lipid core, the surrounding aqueous phase and an interfacial region, as schematically shown in Figure 2.5. In such systems, encapsulated molecules generally partition and may dynamically exchange between the available phases. In the absence of driving forces, (temperature, chemical potential), an equilibrium is reached where no net mass transfer occurs between the phases. The molecular structure and -related to that- affinity for oil and water, determine how components distribute over the phases. The region where the solubility is highest is the energetically preferred one, and the majority of the molecules will be located there, and in the absence of kinetic barriers, polar components will mostly be in the aqueous region, and non-polar ones in the oil, although small amounts with be present in the other phase. Amphiphilic molecules partition in all phases, but tend to locate at the 
interface, and when present in excess start forming micelles when a critical concentration is exceeded in solution.

To describe the distribution, or partitioning, of components between two phases $x$ and $y$ the partition coefficient $K_{o w}$, is used, which is based on the concentration ratio $c_{x} / c_{y}$ in dilute systems (Equation 2.3).

$$
\mathrm{K}_{\mathrm{OW}}=\mathrm{c}_{0} / \mathrm{c}_{\mathrm{w}}
$$

with $c_{o}$ being the concentration in oil and $c_{w}$ the concentration in water. Molecules that are non-polar have a $K_{O W}$ value of above 1 , and the opposite is true for polar components.

As a reference, often the $\log \mathrm{P}$ value is used to describe the relative polarity of a molecule in a water-octanol mixture; with $P$, the octanol-water partition coefficient at a specified temperature. This $P$ value can be measured or calculated based on the molecular structure of a component (Moriguchi et al. 1992, Wang et al. 1997). In real systems, partitioning is much more complex, because also components come into play that may bind part of the component, but in general the $\log \mathrm{P}$ value is a first indication of a component's behaviour.

\section{Aqueous phase}

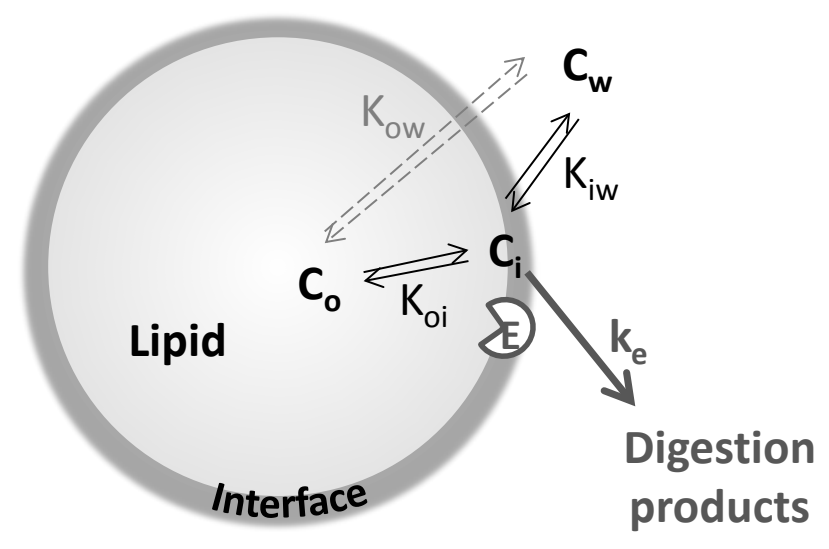

Figure 2.5. Schematic representation of the equilibria between the aqueous phase (w), interface region (i) and lipid droplets (o) for an encapsulate, along with the digestion by enzyme E. The reaction constant ke describes the rate of digestion by that enzyme. $\mathrm{C}_{\mathrm{x}}$ is the concentration of component $\mathrm{C}$ in the region $x$. $K_{n m}$ is the partition coefficient of $C$ over the regions $n$ and $m$, and represents $C_{n} / C_{m}$ (Equation 2.3). 


\section{$\underline{\text { Accumulation at the interface }}$}

Even though the volume of the interface region in general is small compared to the volumes of the oil and aqueous phases, it can have a significant influence on the partitioning of encapsulated ingredients (McClements 2005). In fact, three different partition coefficients have to be defined: oil-water, oil-interface, and interface-water. The logP only describes the overall affinity between oil and water, and does not give information about the propensity of molecules to accumulate at the oil-water interface. In order to predict the interfacial fraction of certain components in emulsions (mainly in equilibrated systems) some techniques have been developed:

- Front-surface fluorescence spectroscopy: This technique can be used to assess the partitioning of proteins in emulsions, to evaluate the modifications upon aging or the displacement of proteins by surfactants (Granger et al. 2005, Rampon et al. 2001, 2003).

- The pseudophase kinetic model has been developed to assess partitioning of antioxidants. All components are assumed to be in dynamic equilibrium between the oil, interface and water. The antioxidant distribution can then be described by the partition constant between the interface and water, and the partition constant between the interface and oil, which can be estimated by using two kinetic data sets (Gunaseelan et al. 2006, Losada-Barreiro et al. 2013).

- Fluorescence microscopy: This technique can be used to visually assess the partitioning of fluorescent probes in multiphase systems (Tikekar \& Nitin 2011).

- Electron paramagnetic resonance: This technique can be used to assess the partitioning of spin probes (model ingredients) between environments of various polarities (Berton-Carabin et al. 2013, Yucel et al. 2013).

\section{$\underline{\text { Short timescale dynamics }}$}

Multiphase systems (such as emulsions and capsules) are not static but continuously exchange molecules between the available regions, even at equilibrium. This implies that any transport from one compartment to another has to be counterbalanced by the reverse transport. The rate of exchange depends on the mass transfer of molecules through the different regions, implying that this can greatly contribute to reactions occurring in the systems (or in the prevention thereof) particularly when the diffusivity of (small) molecules is much faster than the chemical reactions they are involved in (Chaprenet et al. 2014). Chaprenet et al. reported that a small hydrophobic spin probe partitioned mostly in the oil droplet core ( 75\%), but diffused very fast between the oil droplets and the surrounding aqueous phase - much faster than its rate of reduction by ascorbate. Varying the composition and structure of the oil-water interface did not have any effect on the probe reduction rate 
(Chaprenet et al. 2014). This could also explain why, in some studies, interfacial layers designed to reduce lipolysis have not been functional in that respect. Since lipase converts the lipid substrate (triacylglycerols) into more polar products (free fatty acids, mono- and diacylglycerols); these components may partition into the aqueous phase, and without a diffusion barrier thermodynamics will drive mass transfer towards the aqueous phase, and lipolysis at the interface continues.

\section{Longer-term evolution of the system}

Emulsion systems are also subjected to changes over longer timescales than described in the previous paragraph, due to slow migration mechanisms and gradual reorganisation in the system, such as Oswald ripening and compositional ripening. This typically takes minutes to days or even longer, and it is safe to say that many (food) systems are actually metastable (Walstra 2003), and changes in interfacial composition will occur as function of time. The extent of this depends on the components that are used and the processing conditions they were subjected to. For example, homogenization pressure and temperature were shown to affect protein partitioning, and with that the oxidative stability of emulsions in time (Horn et al. 2013). The sequence of ingredient addition can also affect their long-term localization within emulsions, as shown for proteins and phospholipids of which the composition at the oil-water interface (after $48 \mathrm{~h}$ storage) was different when proteins were added before homogenization, and phospholipids added afterwards; or vice versa (Waninge et al. 2005). Besides, chemical degradation of certain components, e.g., by lipid oxidation, may lead to surface-active molecules that cause a change in interfacial organisation (Berton-Carabin et al. 2014).

It has been postulated that multilayered interfaces can act as physical barriers; however, after reviewing the reduction of lipolysis, it can be concluded that most structures do not sufficiently protect. Probably, this can be explained by the same mechanism discussed above for low molecular weight molecules for which the short-timescale diffusion is faster compared to the digestive reactions (Chaprenet et al. 2014). Lipase converts the substrate into more polar low molecular weight products, and they most likely partition more into the aqueous phase in the form of micelles. Without barrier against diffusion across the interface, thermodynamics will drive mass transfer, and lipolysis continues. Only if lipase action is completely prevented (by physical exclusion from the interface) capsules may be not affected by the above mentioned effect.

From all the above, it is clear that partitioning is a factor that can explain some of the findings reported in literature; however, since it was hardly ever investigated as such, it is hard to 
pinpoint the results to this phenomenon, also because this is a very complex factor that changes dramatically during passage in the GI tract.

\subsection{DELAYED LIPOLYSIS TO INDUCE SATIETY}

Considering the factors that affect lipolysis, various strategies may be applied to formulate lipolysis-resistant edible matrices. Here we go beyond the basic, well-established trends that we previously described, namely that lipolysis is slower for longer FAs (Giang et al. 2015, Joyce et al. 2014, Marze et al. 2014) and for higher degree of saturation (McClements et al. 2009); and that the rate of lipolysis is related to the available amount of interfacial area, and hence droplet size (Singh et al. 2009). Systems that aimed at delayed lipolysis are described in this section. We believe that delayed lipolysis can allow targeted delivery of unabsorbed lipids in the small intestine. As described in section 3, sensing of unabsorbed nutrients in the ileum might induce distal to proximal negative feedback, including prolonged gastric retention, which induces satiety.

Matrix large-scale structure. Reducing lipolysis has been achieved both in vitro and in vivo with the use of filled hydrogels (Li et al. 2012). In this study, the authors used whey proteinstabilized oil droplets that were trapped within alginate hydrogel beads $\left(d_{43}=510 \mu \mathrm{m}\right)$, which led to a delayed lipolysis (7\% FFA release after $30 \mathrm{~min}$ ) compared to the non-trapped emulsion $\left(d_{43}=0.36 \mu \mathrm{m}\right)$ or emulsion droplets with alginate bilayer $\left(d_{43}=4.66 \mu \mathrm{m}\right)$ that both released about 68\% FFA after $30 \mathrm{~min}$. By entrapping the emulsion into the gel, the interfacial area of the emulsion remains constant for a longer time, and the effective diffusion distance of lipase is increased. This would also explain that Li et al. (2012) measured a lower lipolysis (19\% FFA release after $30 \mathrm{~min}$ ) after making large clusters $\left(d_{43}=200 \mu \mathrm{m}\right)$ of bilayer emulsion droplets. Besides, it has been reported that alginate can inhibit lipase, via direct interaction with the enzyme or with the substrate at the oil-water interface to directly inhibit the enzyme, or indirectly via interaction with mucin forming a gel, especially when the guluronic acid/manuronic acid ratio is high (Wilcox et al. 2014). Recently, the same research group investigated filled hydrogels in more detail: they improved the preparation method (Matalanis \& McClements 2013), reported pH-dependent hydrogel particles that are only stable at $\mathrm{pH} 4-5$, so could be applied to release in the mouth (Zhang et al. 2015) and they studied the effect on the lipid digestion of such hydrogels again (Mun et al. 2015). Mun and co-workers found a 13-53\% lower initial digestion rate of mung bean-based filled hydrogel and a 16-20\% higher final lipolysis compared to the corresponding emulsion, but these effects were not found with rice-starch-based filled hydrogels.

Organogels are liquid oils structured in the form of gels by phytosterols, waxes, fibres, phospholipids, etc. Phytosterol-structured oil was found to resist in vitro lipolysis more as 
compared to the corresponding non-structured vegetable oil (Duffy et al. 2009). The authors gave three possible explanations for this: oil structuring would slow down substrate diffusion to the interface; micellar tubes would incorporate TAGs; or sterol would inhibit lipase action. Besides, oil structuring might prevent droplet size reduction that does take place under the action of bile salts in liquid fat, resulting in a constant area for lipolysis instead of an increased surface area. Which of these effects is ruling is not fully understood, but it is clear that lipolysis can also be affected by strategies other than interface structuring.

Oil-water interface. A number of components that can locate at the oil-water interface, or alter its composition and structure may be used to influence lipolysis. The effects we describe go beyond the effect of available surface that has been previously touched upon.

Emulsifiers. As discussed previously, components that are present in an interface, such as monoacylglycerols and phospholipids, can influence lipase activity, and this is also true for other emulsifiers. Emulsifiers are defined here as amphiphilic compounds that tend to adsorb at the oil-water interface, lower the interfacial tension, and thereby kinetically stabilize the emulsion. In Supplementary Table 2.2, we have compiled a number of studies for O/W emulsions. The galactolipid digalactosyldiacylglycerol (DGDG) can inhibit bile salt adsorption via steric hindrance, which delayed lipolysis and reduced the reaction rate compared to lecithin from egg yolk (Chu et al. 2009). This effect was not observed with monogalactosyldiacylglycerol (MGDG). The same research group also compared DGDG to dipalmitoylphosphatidylcholine (DPPC), and reported that DGDG-stabilized interfaces were more resistant against adsorption of bile salts, colipase and lipase (Chu et al. 2010). They hypothesize that a certain amount of headgroup-free interfacial area has to be filled with bile salts, and thereby create large enough patches to allow colipase to adsorb, and subsequent lipase action. The larger headgroup of DGDG would prevent the creation of large patches of bile salts via steric hindrance, and would result in more scattered, smaller and discontinuous patches that are considered less effective (Chu et al. 2010).

Similar effects were found when the interfacial behaviour of a non-ionic, brush-like emulsifier (poloxamer Pluronic F68) was compared with an anionic, more compact one (phospholipid Epikuron 145V) at different bile salt concentrations, using interfacial tension and dilatational rheology studies (Torcello-Gómez et al. 2012). The authors found that when Pluronic was used, the rate and extent of bile salt adsorption was lower. This is in agreement with previous findings of the same research group on emulsion stability; the bile salt concentration at which destabilization (reversible phase separation, most likely due to flocculation) occurred is higher for Pluronic, and destabilization proceeded slower, as compared to emulsions made with Epikuron (Jódar-Reyes et al. 2010). 
Surfactant charge can also influence lipolysis, as can be concluded from a study in which nine different surfactants were tested. It showed that non-ionic surfactant-stabilized emulsions have a lower in vitro lipolysis rate than anionic surfactant-stabilized ones (Speranza et al. 2013). The HLB and alkyl chain length of surfactants were also shown to affect the rate of lipolysis and the bioaccessibility of the core oil (measured as the amount of FAs generated in the jejunum, ileum and ileum efflux). The authors found that the rate of lipolysis in the jejunum was lower when higher HLB-surfactants were used, but the bioaccessibility of the FAs from the emulsified oil was higher due to a longer induction time (Speranza et al. 2013). The relation between HLB and lipolysis rate can be explained as follows: surfactants with a high HLB have a large headgroup located on the aqueous phase side, where they may give protection against adsorption of bile salt and lipase. Such high HLB surfactants form micelles more easily, accounting for improved bioaccessibility.

The addition of a low amount of co-surfactant ( $0.25 \mathrm{wt} \%$ monoacylglycerol) to a proteinstabilized emulsion ( $1 \mathrm{wt} \%$ caseinate, $20 \mathrm{wt} \%$ canola oil) resulted in a 2 -fold reduction in lipolysis of emulsified liquid oil, which was explained by easier coalescence of droplets due to reduced charge effects, therewith reducing the available oil-water interfacial area (Day et al. 2014). Addition of more monoacylglycerol ( $0.5 \mathrm{wt} \%$ or $0.75 \mathrm{wt} \%$ ) did increase lipolysis compared to emulsions stabilized with caseinate only, due to increased access of lipase to the surface (Day et al. 2014). The addition of Tween 20 as co-surfactant has also been described to facilitate in vitro lipid digestion of WPI stabilized emulsions rather than reduce it, but in this case also the 4-time smaller droplet size and consequently higher interfacial area, may have played a role (Li \& McClements 2014a). The presence co-surfactant in the primary emulsion also affected the lipolysis of emulsions with additional layers of alginate ( $\mathrm{Li} \&$ McClements 2014a), or alginate and chitosan (Li \& McClements 2014b). Clearly, various effects can be expected when working with co-surfactants.

Interfacial structures extracted from nature have also been investigated with respect to lipolysis. Thylakoids (membranes of chloroplasts from green leaves) were found to inhibit hydrolysis of emulsified oil by lipase/colipase, also in the presence of bile salts (Albertsson et al. 2007). To relate this to either binding of the membrane to the oil-water interface, reducing the access of the lipase/colipase complex or by binding of the membranes to the lipase/colipase complex, blocking the active site of the enzyme. Also in vivo, Albertsson et al. found a suppressed food intake when such membranes were added to rat food.

Another natural interfacial structure that has been investigated for its behaviour during gastrointestinal lipolysis is that of oil bodies (Beindorff et al. 2007, Gallier \& Singh 2012, Gallier et al. 2013, Makkhun et al. 2015). Oil bodies are fat storage structures in seeds, composing of a TAG core in a shell of phospholipids and proteins (e.g., oleosin) (Makkhun 
et al. 2015). A natural emulsion of oil bodies can be obtained by an aqueous extraction from several raw materials, such as sunflower seeds, walnut, almond, and maize. Such oil bodies have been described to induce a feeling of satiety, especially when the proteins are crosslinked, by providing resistance against human digestion (Beindorff et al. 2007). In washed sunflower seed oil bodies, the oleosin has been described to be easily replaced by bile salts, but, the presence of a (natural) protein layer provided protection (Makkhun et al. 2015). Also almond proteins of the oil body membrane have been described provide some protection under in vitro digestion, as they are pepsin-resistant (Gallier \& Singh 2012), whereas the almond oil body emulsion behaved comparable under in vitro digestion to conventional protein-stabilized emulsions. Such pepsin-resistant peptides were also described to be present on walnut oil bodies, and suggested to play a major role in the lipid digestion (Gallier et al. 2013).

Colloidal particles. Instead of conventional emulsifiers, also particles can be used to stabilize the oil-water interface, forming so-called Pickering stabilized interfaces, of which the effect on lipolysis is listed in Supplementary Table 2.3. It has been mentioned that due to a stronger irreversible adsorption of the particles at the oil droplet surface, they are able to reduce the extent and rate of lipolysis more as compared to conventional emulsifier-stabilization (Tzoumaki et al. 2013, Yang et al. 2014a). Chitin nanocrystal-stabilized emulsions showed slower and lower lipolysis compared to conventional emulsions stabilized with whey protein isolate (WPI) and caseinate (Tzoumaki et al. 2013), although the comparison should be treated with care, since different components were used. Emulsions stabilized by particles of Ginkgo biloba extracts or their flavonoid glycosides fraction also showed a lower rate and extent of lipolysis, compared to conventional Tween 20-stabilized emulsions (Yang et al. 2014a). For lactoferrin (LF) particles mixed results were obtained. Lactoferrin nanoparticles (NP) did not affect lipolysis markedly compared to native LF-stabilized emulsions (Meshulam \& Lesmes 2013), but when combined alginate this resulted in a $14 \%$ reduced lipolysis, while with carrageenan an increased lipolysis by $10 \%$ was found (Meshulam \& Lesmes 2013). This may however have been a direct effect of the alginate that can inhibit lipase directly (Wilcox et al. 2014). In conclusion, there is a possible effect of particles, but before drawing any conclusions, this would need to be investigated in much more detail.

Multilayers. In Supplementary Table 2.4 various studies are listed that use more than one layer to influence lipolysis. However, before going into detail, it should be mentioned, that trying to apply multiple layers can be rather tricky, also in view of the previously mentioned competitive adsorption effects. The results are in general rather difficult to interpret as no clear trends were observed, but we still try to discuss them shortly. 
Addition of beet pectin to a WPI-stabilized emulsion was reported to reduce lipolysis (Xu et al. 2014), both when WPI and pectin were conjugated first or adsorbed subsequently, and this could indicate that an interlinked layer is formed that has synergistic effects, or as the authors prefer to think that unadsorbed pectin may bind to lipase or bile salts therewith reducing lipolysis. In another study, the addition of a chitosan layer on top of a $\beta$ lactoglobulin (BLG)-stabilized emulsion did reduce lipolysis, and a third layer of either alginate or pectin reduced lipolysis slightly further (Li et al. 2010). In a third study, when using a primary caseinate-stabilized emulsion at low $\mathrm{pH}$ and adsorbing a chitosan layer by increasing the $\mathrm{pH}$ to 5, the lipolysis was also reduced (from $90 \%$ to $75 \%$ after 15 min of digestion) (Hu et al. 2010b). However, when adding a third pectin layer, the authors found the lipolysis much less reduced as compared to the bilayered emulsion. Besides, only minor reduction in lipolysis was observed when adsorbing a pectin secondary layer by lowering the $\mathrm{pH}$ from 7 to 4.5, and adding a third chitosan and fourth pectin layer, which even enhanced lipolysis (Hu et al. 2010b). The addition of a chitosan third layer onto a secondary emulsion of alginate on top of BLG also increased the lipolysis rather than reducing it (Li \& McClements 2014b).

It is obvious that the choice of components and the conditions under which they are used are critical in order to be successful in reduction in lipolysis. More precisely, the ultrastructure, and in particular the porosity of the interfacial layer, which defines the accessibility of the core lipid to lipase, need to be considered. Joyce and co-workers investigated various porous structures, and found that the diameter of the pore relative to the size of lipase is important (Joyce et al. 2014). Digestion will be inhibited when the pores are small enough to hinder lipase to enter, and a pore size just larger than the diameter of lipase has been described to enhance its action (Joyce et al. 2014). Also some effects were dedicated to the hydrophobicity/hydrophilicity of the porous structure in this work, but it is not clear whether these effects can be solely contributed to that, or that the pore size also influenced these findings.

The barrier properties for lipase can be improved when a more cohesive layer at the interface is formed; heating starch particle- stabilized emulsions decreased lipase activity up to $60 \%$ at the temperature where starch gelatinized enough to create a dense layer but not swelled further into a more loose structure more accessible for lipase to penetrate (Sjöö et al. 2015, Timgren et al. 2011). Another way to improve the barrier properties of the interface is crosslinking interfacial biopolymers; cross-linking interfacial casein with the functional ingredient genipin enhanced acid stability and delayed in vitro digestibility (Hu et al. 2015). But, crosslinking does not essentially improve the barrier properties, as in vitro lipid digestion was not greatly affected by cross-linking an interfacial pectin layer with laccase (Zeeb et al. 2015). 
All effects described above in relation to interfacial structure, suggest that characterizing the ultrastructure and morphology of oil-water interfaces, at scales relevant to the dimensions of a lipase molecule, could be a viable strategy to assess $a b$ initio how fast and to which extent lipolysis is likely to occur in $\mathrm{O} / \mathrm{W}$ emulsions.

Clinical trials. One available product that is claimed to induce satiety by reducing lipolysis is Olibra ${ }^{\circledR}$ (Lipid Technologies Provider AB, Sweden). This is an emulsion of fractionated palm / oat oil that naturally contains galactolipids. Diepvens et al. (2007) provided Olibra ${ }^{\circledR}$ twice daily for 26 weeks in a yogurt (per serving $5 \mathrm{~g}$ fat, of which $40 \%$ from the vegetable oils) and found an improved weight management after an initial weight loss period in overweight women, as compared to providing a placebo (5 g milk fat per serving). This indicates the absence of compensation behaviour during long-term use. In another human intervention study, the commercially available yogurt Fabuless ${ }^{\circledR}$ (DSM Food Specialities, Netherlands, also containing palm and oat oil, previously called Olibra ${ }^{\circledR}$ and Reducal ${ }^{\circledR}$, with $28.3 \mathrm{~g}$ total fat) resulted in a 2-fold higher amount of undigested lipids in the jejunum compared to the control (with 28.3 g milk fat) (Knutson et al. 2010). They observed needleshaped fat crystals in the jejunal samples from subjects that consumed the test samples, which were not seen in the control group. Knutson et al. suggest that galactolipids from oat oil would play a crucial role in the formation of crystals from palm oil. These crystals are proposed to be digested slower, so the ileum is longer exposed to fatty acids.

The effectiveness of this type of product to induce satiety via activation of the 'ileal brake' is still not completely confirmed. The intervention study of Smith et al (2011) did not show an effect of Fabuless ${ }^{\circledR}$ on appetite, and only a weak effect on food intake. Nevertheless, the industry does see a potential in this concept as several patents are based on satiety emulsions (Bialek et al. 2006, Golding et al. 2009, Herslof et al. 2003).

\subsection{CONCLUSIONS}

Dietary lipids have been encapsulated using many different processes and construction materials, but the performance regarding reduction of lipolysis is overall poor and varies considerably. The fact that the results show only minor effects and are not consistent indicates that some key factors in the process of GI lipolysis are not yet fully understood and hence cannot be sufficiently controlled to induce satiety. The non-consistency in results also stems from the in vitro models that varied greatly; hopefully the standardised method that is now agreed upon will help in that respect. Still, this method can only give a limited impression of the effects that are generated since it does not include biological feedback routes that determine lipolysis and its effect on satiety. 
Until present, only one type of product has been commercialized to induce satiety via lipid signalling in the distal small intestine, but its effectiveness is controversial. Therefore we conclude that it is challenging to delay lipolysis; and that would be even more the case for release of lipids in the more distal parts of the small intestine where they can induce satiety.

In our view the most promising route to achieve delayed lipolysis is by designing the structure and morphology of the oil-water interface, at scales relevant to the dimensions of a lipase molecule. In that way, lipase would get controlled access to the oil/water interface. This is far from trivial as stated in this review, and most probably multidisciplinary teams are needed to link medical and technical challenges, but at the same time the advantages will be great since such capsules could provide a strategy for non-invasive long term weight management. 


\subsection{SUPPLEMENTARY INFORMATION}

Supplementary Table 2.2. Overview of studies on the effect of emulsifier interfaces on lipolysis.

\begin{tabular}{|c|c|c|c|c|c|c|c|}
\hline & Reference & Emulsifier & Oil & $d_{32}(\mu \mathrm{m})$ & In vitro digestion conditions & $\begin{array}{c}\text { Characteristic } \\
\text { times for digestion } \\
*\end{array}$ & Key findings \\
\hline \multirow{19}{*}{ 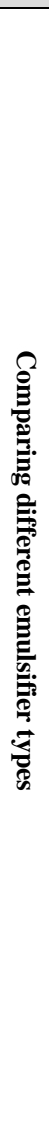 } & \multirow[t]{4}{*}{ (Mun et al. 2007) } & cas & \multirow{4}{*}{$\begin{array}{l}3 \text { wt\% corn oil in } \\
\text { water }\end{array}$} & 0.35 & \multirow{4}{*}{$\begin{array}{l}1.6 \mathrm{mg} / \mathrm{ml} \text { lipase, } 5 \mathrm{mg} / \mathrm{ml} \\
\text { bile extract }\end{array}$} & $3.7 / 120 ; 263$ & \multirow{4}{*}{$\begin{array}{l}\text { Lipolysis of emulsion stabilized } \\
\text { by proteins (cas and WPI) > } \\
\text { phospholipids (lecithin) > non- } \\
\text { ionic surfactants (Tween 20) }\end{array}$} \\
\hline & & wpi & & 0.27 & & $3.2 / 120 ; 241$ & \\
\hline & & lec & & 0.3 & & $2.7 / 120 ; \underline{158}$ & \\
\hline & & $\mathrm{T} 20$ & & 0.31 & & 29.9/120;168 & \\
\hline & \multirow[t]{4}{*}{ (Hur et al. 2009) } & lec & \multirow[t]{4}{*}{3 wt\% soybean oil } & $3.8 \& 1.4$ & \multirow{4}{*}{$\begin{array}{l}5 \text { min in saliva juice, } 2 \mathrm{~h} \text { in } \\
\text { gastric juice, } 2 \mathrm{~h} \text { in duodenal } \\
+ \text { bile juice }\end{array}$} & \multirow{4}{*}{$\begin{array}{l}\text { They were unable to } \\
\text { obtain reliable } \\
\text { results, due to } \\
\text { interference of } \\
\text { components of the } \\
\text { simulated digestion } \\
\text { media. }\end{array}$} & \multirow{4}{*}{$\begin{array}{l}\text { Only limited effect of the } \\
\text { emulsifier on microstructure } \\
\text { changes during digestion. }\end{array}$} \\
\hline & & $\mathrm{T} 20$ & & $5.9 \& 2.1$ & & & \\
\hline & & wpi & & $4.6 \& 2$ & & & \\
\hline & & cas & & $4.2 \& 1.5$ & & & \\
\hline & \multirow{5}{*}{$\begin{array}{l}\text { (Golding et al. } \\
\text { 2011) }\end{array}$} & lec & \multirow[t]{4}{*}{20 wt $\%$ canola oil } & 0.25 & \multirow{5}{*}{$\begin{array}{l}2 \mathrm{~h} \text { incubation simulated } \\
\text { gastric fluid ( } 2 \mathrm{mg} / \mathrm{ml} \mathrm{NaCl} \text {, } \\
0.4 \mathrm{mg} / \mathrm{ml} \text { pepsin, } 0.2 \mathrm{mg} / \mathrm{ml} \\
\text { lipase, pH 1.9), 3h in } \\
\text { simulated intestinal fluid (10 } \\
\mathrm{mM} \mathrm{CaCl}_{2}, 12 \mathrm{mM} \text { mixed bile } \\
\text { salts, } 0.75 \mathrm{mM} \text { phospholipid, } \\
150 \mathrm{mM} \mathrm{NaCl,} 4 \mathrm{mM} \text { Tris- } \\
\mathrm{HCL}, 125 \mathrm{mg} / \mathrm{ml} \text { pancreatin, } \\
\text { pH 6.8) }\end{array}$} & $5 / 180 ; 256$ & \multirow{5}{*}{$\begin{array}{l}\text { Extent of lipolysis phospholipid, } \\
\text { tween, wpi comparable, only } \\
\text { slightly slower initial rate tween } \\
\text { and wpi. SSL slowed lipolysis } \\
\text { down and reduced it, especially } \\
\text { when the oil core was partly solid. }\end{array}$} \\
\hline & & $\mathrm{T} 80$ & & 0.38 & & $7 / 180 ; 260$ & \\
\hline & & wpi & & 0.41 & & $7 / 180 ; 253$ & \\
\hline & & SSL & & 0.34 & & $\underline{16} / 180 ; \underline{144}$ & \\
\hline & & SSL & $\begin{array}{l}20 \text { wt } \% \text { canola } \\
\text { oil+hydrogenated } \\
\text { vegetable oil (25\% } \\
\left.\text { SFC at } 37^{\circ} \mathrm{C}\right)\end{array}$ & 0.34 & & $\underline{46} / 180 ; \underline{128}$ & \\
\hline & \multirow[t]{4}{*}{ (Keogh et al. 2011) } & lec & \multirow[t]{2}{*}{ liquid } & & \multirow{4}{*}{$\begin{array}{l}\text { in vitro lipolysis, in vivo } \\
\text { plasma TG, CCK, GLP, PYY } \\
\text { levels, feelings of hunger and } \\
\text { satiety }\end{array}$} & $1.8 / 180,255$ & \multirow{4}{*}{$\begin{array}{l}\text { Comparing emulsions with the } \\
\text { same core, SSL lower rate and } \\
\text { extent of lipolysis than lecithin and } \\
\text { cas. Higher solid fat content } \\
\text { slowed down and reduced } \\
\text { digestion. }\end{array}$} \\
\hline & & SSL & & & & $3.1 / 180 ; \underline{143}$ & \\
\hline & & SSL & \multirow[t]{2}{*}{ liquid and solid } & & & $\underline{48} / 180 ; \underline{126}$ & \\
\hline & & cas/mag & & & & $\underline{35} / 180 ; \underline{194}$ & \\
\hline & \multirow[t]{2}{*}{$\begin{array}{l}\text { (Torcello-Gómez } \\
\text { et al. 2011) }\end{array}$} & P68 & \multirow[t]{2}{*}{$3 \%$ olive oil } & $\begin{array}{l}5 \text { or } \\
20\end{array}$ & \multirow{2}{*}{$\begin{array}{l}1.6 \mathrm{mg} / \mathrm{ml} \text { lipase, } 5 \mathrm{mg} / \mathrm{ml} \\
\text { bile extract, } 20 \mathrm{mM} \mathrm{CaCl}_{2}, \mathrm{pH} \\
7.0\end{array}$} & 11/120;74 23 $3 / 120 ; \underline{69}$ & \multirow{2}{*}{$\begin{array}{l}\text { Lipolysis rate and extent lower for } \\
\text { nonionic poloxamer stabilized } \\
\text { emulsion than for lec-stabilized. }\end{array}$} \\
\hline & & lec & & $\begin{array}{l}10 \text { or } \\
15\end{array}$ & & $\begin{array}{l}6 / 120 ; 80 \\
11 / 120 ; 93\end{array}$ & \\
\hline
\end{tabular}


Supplementary Table 2.2. Continued.

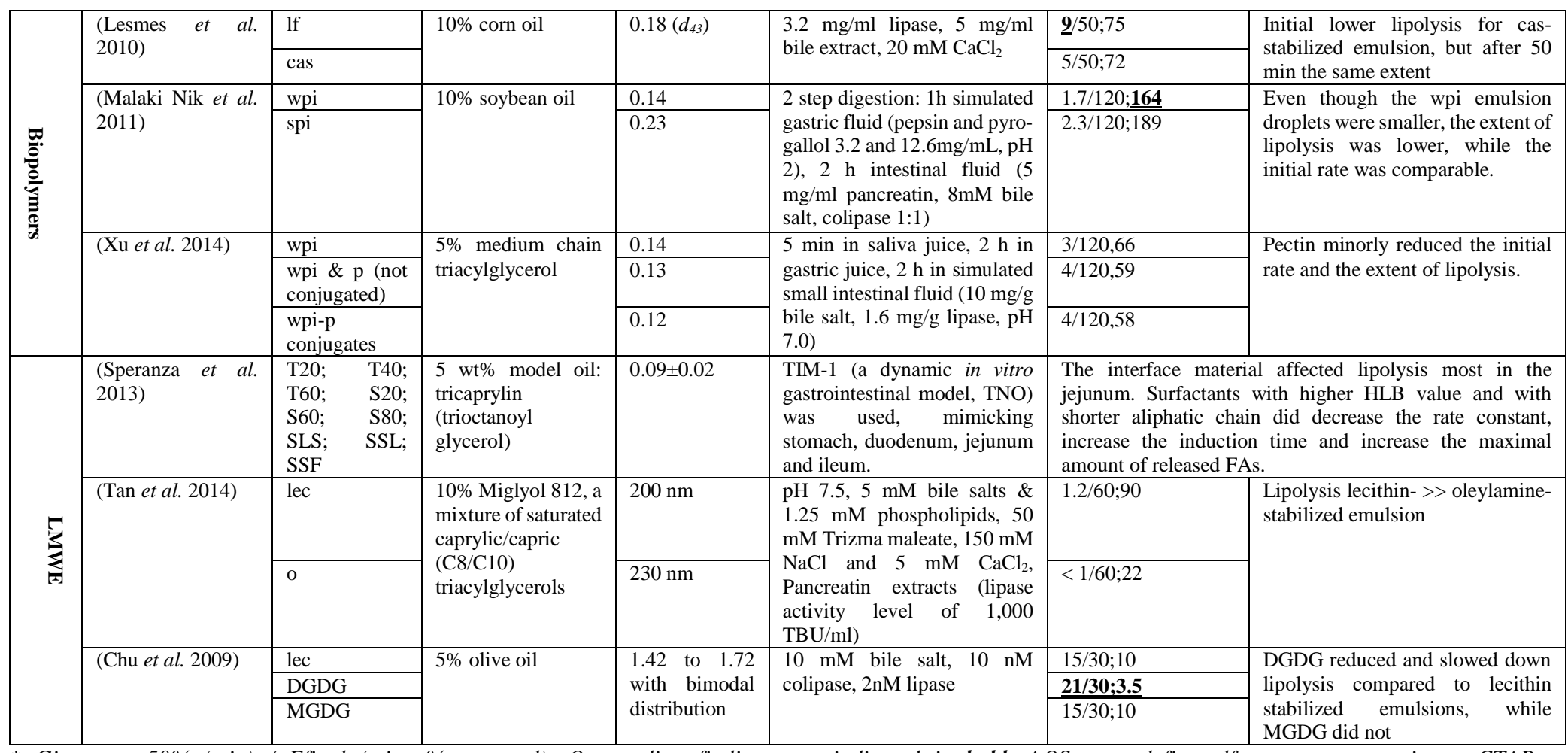

* Given as $150 \%$ (min) / Efinal (min; \% or umol). Outstanding findings are indicated in bold. AOS = $\alpha$-olefin sulfonate, cas =caseinate, CTAB = cetyltrimethylammonium, $D T A B=$ dodecyltrimethylammonium bromide, E145 = the phospholipid Epikuron $145 \mathrm{~V}$, lec $=$ lecithin, lf $=$ lactoferrin, mag $=$ monoacylglycerol, $o=$ oleylamine, $p=$ pectin, $P 68=$ the surfactant pluronic F68, SLES = sodium lauryl ether sulphate, SLS = sodium lauryl sulphate, spi = soy protein, SSF = sodium stearyl fumarate, SSL = sodium stearoyl lactylate, S20, 60, 80 = sorbitan monolaurate (Span 20), sorbitan monostearate (Span 60), sorbitan monooleate (Span 80), T20, 40, 60, 80 = polyoxyethylenesorbitan monolaurate (Tween 20), polyoxyethylenesorbitan monopalmitate (Tween 40), Polyoxyethylene sorbitan monostearate (Tween 60), polyoxyethylene sorbitan monooleate $($ Tween 80$)$, wpi $=$ whey protein. 
Supplementary Table 2.3. Lipolysis of particle-stabilized emulsions

\begin{tabular}{|c|c|c|c|c|c|c|c|c|c|}
\hline Reference & $\begin{array}{l}\text { Particle } \\
\text { type }\end{array}$ & $\begin{array}{c}\text { Particle } \\
\text { size }(\mathrm{nm})\end{array}$ & $\begin{array}{c}\text { Particle } \\
\text { charge } \\
(\mathrm{mV})\end{array}$ & $\begin{array}{l}\text { Lipid } \\
\text { phase }\end{array}$ & $\begin{array}{c}\text { Aqueous } \\
\text { phase }\end{array}$ & $\begin{array}{c}\text { Emulsion } \\
\text { droplet } \\
\text { size }(\mu \mathrm{m}) \\
\end{array}$ & $\begin{array}{l}\text { In vitro digestion } \\
\text { conditions }\end{array}$ & $\begin{array}{c}\text { Characteristic } \\
\text { times for } \\
\text { digestion* }\end{array}$ & Key findings \\
\hline $\begin{array}{l}\text { (Meshulam } \\
\text { \& Lesmes } \\
\text { 2013) }\end{array}$ & $\begin{array}{l}\text { If NP, bare } \\
\text { or coated } \\
\text { with a or cr }\end{array}$ & $\begin{array}{l}200-400 \\
\text { (bare); up } \\
\text { to } 1600 \\
\text { (coated) }\end{array}$ & $\begin{array}{l}+22 \\
\text { (bare) to } \\
-75 \\
\text { (coated) }\end{array}$ & $\begin{array}{l}2 \% \text { olive } \\
\text { oil }\end{array}$ & $\begin{array}{l}0.2 \% \\
\text { suspended in } \\
\text { water, } \mathrm{pH} 7-8\end{array}$ & $1-10$ & $\begin{array}{l}\text { 5mM phosphate buffer } \mathrm{pH} \\
7,5 \mathrm{mg} / \mathrm{ml} \mathrm{bile} \mathrm{extract,} \\
20 \mathrm{mM} \mathrm{CaCl}_{2}, 590 \mathrm{U} / \mathrm{ml} \\
\text { lipase }\end{array}$ & $\begin{array}{l}\text { native: } 14 / 120 ; 67 \\
\text { lf np: } 16 / 120 ; 69 \\
\text { lf-a: } 11 / 120 ; 58 \\
\text { lf-cr: } 8 / 120 ; 73\end{array}$ & $\begin{array}{l}\text { If NP and native lf } \\
\text { comparable effect on in } \\
\text { vitro intestinal digestion; } \\
\text { lipolysis } 14 \% \text { reduced by } \\
\text { a- coated NP and } 10 \% \\
\text { increased by cr-coated } \\
\text { NP }\end{array}$ \\
\hline $\begin{array}{l}\text { (Ruiz- } \\
\text { Rodriguez et } \\
\text { al. 2014) }\end{array}$ & $\begin{array}{l}\text { Fumed } \\
\text { silica } \\
\text { powder } \\
\text { containing } \\
\text { partially } \\
\text { hydrophobis } \\
\text { ed NP } \\
\end{array}$ & - & $\begin{array}{l}-43.5 \mathrm{mV} \\
\text { at } 5 \%,- \\
47.6 \mathrm{mV} \\
\text { at } 0.5 \%\end{array}$ & $\begin{array}{l}5 \% \text { olive } \\
\text { oil }\end{array}$ & $\begin{array}{l}\text { Silica NP } \\
\text { suspended in } \\
10 \text { mM, pH } 7 \\
\text { phosphate } \\
\text { buffer }\end{array}$ & $\begin{array}{l}5 \% \mathrm{NP}: \\
3.7 \\
0.5 \% \mathrm{NP}: \\
8.4\end{array}$ & $\begin{array}{l}\text { 10mM phosphate buffer } \\
\mathrm{pH} 7,5 \mathrm{mg} / \mathrm{ml} \text { bile extract, } \\
20 \mathrm{mM} \mathrm{CaCl}_{2}, 590 \mathrm{U} / \mathrm{ml} \\
\text { lipase }\end{array}$ & $\begin{array}{l}\beta \text {-lg: 8/120;62 } \\
\text { s np: 7/120;54 }\end{array}$ & $\begin{array}{l}\text { Lower extent of lipolysis } \\
\text { in } 1,3 \text { and } 5 \% \text { silica } \\
\text { stabilized emulsions } \\
\text { compared to } 1 \% \beta \text {-lg } \\
\text { stabilized emulsions, but } \\
\text { no difference in initial } \\
\text { lipolysis rate }\end{array}$ \\
\hline $\begin{array}{l}\text { (Simovic et } \\
\text { al. 2009) }\end{array}$ & $\begin{array}{l}\text { Hydrophilic } \\
\text { silica NP }\end{array}$ & & & $\begin{array}{l}10 \% \\
\text { fractiona } \\
\text { ted TG } \\
\text { coconut } \\
\text { oil (with } \\
\text { lec or o) }\end{array}$ & & & $\begin{array}{l}50 \mathrm{mM} \text { TRIS maleate, } 150 \\
\mathrm{mM} \mathrm{NaCl}, 5 \mathrm{mM} \mathrm{CaCl}_{2} \cdot 2 \\
\mathrm{H}_{2} \mathrm{O} \text {, pH } 7.5 \text {, containing } \\
5 \mathrm{mM} \text { NaTDC and } 1.25 \\
\mathrm{mM} \text { PC; pancreatic } \\
\text { extract (lipase } 1,000 \mathrm{TBU} \\
\text { per mL final) }\end{array}$ & $\begin{array}{l}\text { lipid: } 10 / 60 ; 51 \\
\text { lec: 5/60;73 } \\
\text { lec-s: 3/60;100 }\end{array}$ & $\begin{array}{l}\text { Lipolysis improved by } \\
\text { lec hybrid lipid-silica } \\
\text { microcapsules compared } \\
\text { to lec stabilized } \\
\text { submicron emulsions. }\end{array}$ \\
\hline \multirow[t]{2}{*}{$\begin{array}{l}\text { (Tan et al. } \\
\text { 2014) }\end{array}$} & \multirow[t]{2}{*}{ silica } & \multirow[t]{2}{*}{$\begin{array}{l}100 \text { (also } \\
200 \text { or } \\
1,000) \\
\text { nm }\end{array}$} & negative & \multirow{2}{*}{$\begin{array}{l}10 \% \\
\text { Miglyol } \\
812, \text { a } \\
\text { mixture } \\
\text { of } \\
\text { saturated } \\
\text { caprylic/ } \\
\text { capric } \\
\text { (C8/C10 } \\
\text { ) } \\
\text { triacylgl } \\
\text { ycerols }\end{array}$} & $\begin{array}{l}\text { lec in water } \\
\text { during } \\
\text { emulsification, } \\
\text { after that } 5 \\
\text { wt\% s } \\
\text { dispersion } \\
\text { added } \\
\end{array}$ & 200 & \multirow[t]{2}{*}{$\begin{array}{l}\text { pH 7.5, } 5 \text { mM bile salts \& } \\
1.25 \mathrm{mM} \text { phospholipids, } \\
50 \mathrm{mM} \text { Trizma maleate, } \\
150 \mathrm{mM} \mathrm{NaCl} \text { and } 5 \mathrm{mM} \\
\mathrm{CaCl}_{2} \text {, Pancreatin extracts } \\
\text { (lipase activity level of } \\
\text { 1,000TBU/ml) }\end{array}$} & $\begin{array}{l}\text { lec: /60;90 } \\
\text { s hybrid lec: /60;88 }\end{array}$ & \multirow{2}{*}{$\begin{array}{l}\text { The lipolysis of the } \\
\text { negatively charged } \\
\text { emulsions was much } \\
\text { higher than positively } \\
\text { charged ones. Hybrids } \\
\text { made with repulsive } \\
\text { nanoparticle-droplet } \\
\text { interactions (lec) also } \\
\text { enhanced the lipolysis, } \\
\text { while with attractive } \\
\text { interactions (o emulsion) } \\
\text { the lipolysis was } \\
\text { hindered. }\end{array}$} \\
\hline & & & positive & & $\begin{array}{l}\text { o in water } \\
\text { during } \\
\text { emulsification, } \\
\text { after that } 5 \\
\text { wt\% s } \\
\text { dispersion } \\
\text { added }\end{array}$ & 230 & & $\begin{array}{l}\text { o: /60;22 } \\
\text { s hybrid o: /60;62 }\end{array}$ & \\
\hline
\end{tabular}


Supplementary Table 2.3. Continued.

\begin{tabular}{|c|c|c|c|c|c|c|c|c|c|}
\hline $\begin{array}{l}\text { (Timgren et } \\
\text { al. 2011) }\end{array}$ & $\begin{array}{l}\text { OSA starch } \\
\text { particles }\end{array}$ & $\begin{array}{l}600- \\
1,000\end{array}$ & - & $\begin{array}{l}12.5-33 \\
\% \mathrm{MCT} \\
\text { oil }\end{array}$ & $\begin{array}{l}0.2-6 \% \\
\text { suspended in } \\
\text { pH } 7 \text { buffer, } \\
\mathrm{NaCl} 0.2 \mathrm{M}\end{array}$ & $\begin{array}{l}20-50 \\
\left(d_{32}\right)\end{array}$ & $\begin{array}{l}\text { buffer pH7, bile salts, } 3 \\
\mathrm{mg} / \mathrm{ml} \text { lipase, } 1 \mathrm{mg} / \mathrm{ml} \mathrm{co-} \\
\text { lipase }\end{array}$ & \multicolumn{2}{|c|}{$\begin{array}{l}\text { Lipase activity reduced from } 68 \% \text { to } 34 \% \text { by } \\
\text { increasing the amount of starch from } 75 \text { to } 600 \\
\mathrm{mg} / \mathrm{ml} \text {. }\end{array}$} \\
\hline $\begin{array}{l}\text { (Tzoumaki et } \\
\text { al. 2013) }\end{array}$ & ChN & $\begin{array}{l}\text { rods of } \\
20 * 240\end{array}$ & - & $\begin{array}{l}10 \% \\
\text { corn oil }\end{array}$ & $\begin{array}{l}0.01-1 \% \text { ChN } \\
\text { suspended in } \\
\text { water + HCL, } \\
\text { pH } 3\end{array}$ & $10-100$ & $\begin{array}{l}10 \mathrm{mM} \text { phosphate buffer } \\
\mathrm{pH} 7,5 \mathrm{mg} / \mathrm{ml} \text { bile extract, } \\
1.6 \mathrm{mg} / \mathrm{ml} \text { pancreatic } \\
\text { lipase, } 5 \mathrm{mM} \mathrm{CaCl} 2\end{array}$ & $\begin{array}{l}\text { wpi: } 11 / 55 ; 48 \\
\text { cas: } 8 / 55 ; 59 \\
\text { ChN: } 9 / 55 ; 28\end{array}$ & $\begin{array}{l}\text { Lower rate and extent of } \\
\text { lipolysis in emulsions } \\
\text { stabilized by ChN } \\
\text { compared to protein. }\end{array}$ \\
\hline $\begin{array}{l}\text { (Yang et al. } \\
\text { 2014a) }\end{array}$ & $\begin{array}{l}\text { Ginkgo } \\
\text { biloba } \\
\text { extracts } \\
\text { (GBE) and } \\
\text { their } \\
\text { flavonoid } \\
\text { glycosides } \\
\text { fraction } \\
\text { (FA) } \\
\text { particles }\end{array}$ & $\begin{array}{l}800(\mathrm{FA}) \\
\text { and } 1,000 \\
(\mathrm{GBE})\end{array}$ & - & $\begin{array}{l}10 \% \\
\text { soybean } \\
\text { oil }\end{array}$ & $\begin{array}{l}100 \mathrm{mg} \text { FA, } \\
\text { GBE or T20 } \\
\text { dissolved in } \\
90 \mathrm{ml} \text { buffer } \\
(20 \mathrm{mM} \\
\text { bisetris, pH } \\
7.0)(0.02 \% \\
\text { sodium azide) }\end{array}$ & $\begin{array}{l}0.8(\mathrm{FA}) \\
\text { and } 1.0 \\
(\mathrm{GBE})\end{array}$ & $\begin{array}{l}\text { mouth, gastric, small } \\
\text { intestine (pH 7, duodenal } \\
\text { juice, bile juice) }\end{array}$ & $\begin{array}{l}\text { T20: } 2.5 / 10 ; 43 \\
\text { GBE: } 1.3 / 10 ; 22 \text { FA: } \\
0.7 / 10 ; 11\end{array}$ & $\begin{array}{l}\text { FA and GBE stabilized } \\
\text { emulsions much lower } \\
\text { lipolysis compared to } \\
\text { surfactant stabilized. }\end{array}$ \\
\hline
\end{tabular}

* Given as t50\% (min) / Efinal (min; \%). a = alginate, $\beta$-lg = beta-lactoglobulin, $\mathrm{cr}=$ carageenan, If = lactoferrin, ChN = chitin nanocrystals, lec = lecithin, $N P=$ nanoparticle, $o=$ oleylamine, $T 20=$ Tween 
Supplementary Table 2.4. Effect of layered interfaces on lipolysis.

\begin{tabular}{|c|c|c|c|c|c|c|c|c|c|c|c|}
\hline \multirow[b]{2}{*}{ Reference } & \multicolumn{4}{|c|}{ Component used in layer } & \multirow[t]{2}{*}{ Oil phase } & \multirow{2}{*}{$\begin{array}{l}\text { In vitro digestion } \\
\text { conditions }\end{array}$} & \multicolumn{4}{|c|}{ Characteristic times for digestion*, \# layers denoted } & \multirow[t]{2}{*}{ Key findings } \\
\hline & 1 & 2 & 3 & $\geq 4$ & & & 1 & 2 & 3 & 4 & \\
\hline $\begin{array}{l}\text { (Tokle et al. } \\
\text { 2013) }\end{array}$ & If & $\beta-\lg$ & lf & $\beta-\lg$ & $\begin{array}{ll}2 \% & \text { corn } \\
\text { oil } & \end{array}$ & $\begin{array}{l}\text { oral, gastric and small } \\
\text { intestinal stage }(4.8 \\
\mathrm{mg} / \mathrm{ml} \text { lipase, } 5 \mathrm{mg} / \mathrm{ml} \\
\text { bile extract, } 750 \mathrm{mM} \\
\left.\mathrm{CaCl}_{2}\right)\end{array}$ & $7 / 60 ; 101$ & $8 / 60 ; 115$ & $5 / 60 ; 114$ & $8 / 60 ; 102$ & \\
\hline \multirow{2}{*}{$\begin{array}{l}\text { (Hu et al. } \\
2010 \mathrm{~b})\end{array}$} & cas & c & $\mathrm{p}$ & & \multirow{2}{*}{$\begin{array}{l}10 \% \text { corn } \\
\text { oil }\end{array}$} & \multirow{2}{*}{$\begin{array}{l}1.6 \mathrm{mg} / \mathrm{ml} \text { lipase, } \\
5 \mathrm{mg} / \mathrm{ml} \text { bile salt, } 5 \mathrm{mM} \\
\mathrm{CaCl}_{2}\end{array}$} & $1 / 30 ; 89$ & $\underline{5 / 30 ; 77}$ & $2 / 30 ; 85$ & & \multirow{2}{*}{$\begin{array}{l}\text { Only the secondary cas-c } \\
\text { emulsion showed a clear } \\
\text { reduction in rate and extent } \\
\text { of lipolysis }\end{array}$} \\
\hline & cas & $\mathrm{p}$ & C & $\mathrm{p}$ & & & $3 / 30 ; 87$ & $3 / 30 ; 83$ & $5 / 30 ; 87$ & $2 / 30 ; 90$ & \\
\hline \multirow{2}{*}{$\begin{array}{l}(\mathrm{Li} \text { et al. } \\
2010)\end{array}$} & blg & C & $\mathrm{a}$ & & \multirow{2}{*}{$\begin{array}{l}10 \% \text { oil } \\
\text { (corn and } \\
\text { tributyrin } \\
1: 1 \text { ) }\end{array}$} & \multirow{2}{*}{$\begin{array}{l}1.6 \mathrm{mg} / \mathrm{ml} \text { lipase, } 5 \\
\mathrm{mg} / \mathrm{ml} \mathrm{bile} \mathrm{salt,} 5 \text { or } \\
20 \mathrm{mM} \mathrm{CaCl}_{2}\end{array}$} & $7 / 41 ; 117$ & $47 / 120 ; 101$ & $43 / 120 ; 82$ & & \multirow{2}{*}{$\begin{array}{l}\text { Lipolysis (rate and extent) } \\
\text { clearly reduced by adding } 1 \\
\text { or } 2 \text { layers, compared to the } \\
\text { primary emulsion. }\end{array}$} \\
\hline & blg & C & $\mathrm{p}$ & & & & & & $45 / 120 ; 85$ & & \\
\hline \multirow[t]{6}{*}{$\begin{array}{l}\text { (Li \& } \\
\text { McClement } \\
\text { s 2014a,b) }\end{array}$} & \multirow[t]{3}{*}{$\beta-\lg$} & \multirow[t]{3}{*}{$\mathrm{a}$} & \multirow[t]{3}{*}{ C } & & \multirow[t]{6}{*}{$\begin{array}{l}10 \% \text { corn } \\
\text { oil }\end{array}$} & $\begin{array}{l}\text { fasted }(0.53 \mathrm{mg} / \mathrm{ml} \\
\text { lipase, } 5 \mathrm{mg} / \mathrm{ml} \text { bile salt, } \\
\left.5 \mathrm{mmol} / \mathrm{L} \mathrm{CaCl}_{2}\right)\end{array}$ & $9 / 60 ; 53$ & $11 / 60 ; 64$ & $6 / 60 ; 75$ & & \multirow{6}{*}{$\begin{array}{l}\text { No clear trends were } \\
\text { observed in the effect of } \\
\text { additional layers. Under } \\
\text { fed-high Ca conditions, the } \\
\text { lipolysis of } \beta \text {-lg+T20-a-c } \\
\text { emulsions showed a } \\
\text { significant delay. Lipolysis } \\
\text { was higher in fed state } \\
\text { (especially at high Ca), } \\
\text { except for the } \beta \text {-lg-a } \\
\text { emulsion. }\end{array}$} \\
\hline & & & & & & $\begin{array}{l}\text { fed }(2.4 \mathrm{mg} / \mathrm{ml} \text { lipase, } \\
20 \mathrm{mg} / \mathrm{ml} \mathrm{bile} \mathrm{salt,} \mathbf{1 0} \\
\left.\mathrm{mmol} / \mathrm{L} \mathrm{CaCl}_{2}\right)\end{array}$ & 4/60; 96 & $2 / 60 ; 94$ & $2 / 60 ; 97$ & & \\
\hline & & & & & & $\begin{array}{l}\text { fed }(2.4 \mathrm{mg} / \mathrm{ml} \text { lipase, } \\
20 \mathrm{mg} / \mathrm{ml} \mathrm{bile} \mathrm{salt,} 20 \\
\left.\mathrm{mmol} / \mathrm{L} \mathrm{CaCl}_{2}\right)\end{array}$ & $16 / 60 ; 103$ & $0 / 60 ; \underline{34}$ & $12 / 60 ; 89$ & & \\
\hline & \multirow{3}{*}{$\begin{array}{l}\beta- \\
\lg + \\
\mathrm{T} 20\end{array}$} & \multirow[t]{3}{*}{$\mathrm{a}$} & \multirow[t]{3}{*}{ C } & & & fasted & $4 / 60 ; 78$ & $4 / 60 ; 73$ & $2 / 60 ; 64$ & & \\
\hline & & & & & & fed $\left(10 \mathrm{mmol} / \mathrm{L} \mathrm{CaCl}{ }_{2}\right)$ & $2 / 60 ; 95$ & $2 / 60 ; 84$ & $2 / 60 ; 83$ & & \\
\hline & & & & & & fed $\left(20 \mathrm{mmol} / \mathrm{L} \mathrm{CaCl}{ }_{2}\right)$ & $10 / 60 ; 105$ & $16 / 60 ; 93$ & $\underline{\mathbf{4 9}} / 60 ; 81$ & & \\
\hline
\end{tabular}


Supplementary Table 2.4. Continued.

\begin{tabular}{|c|c|c|c|c|c|c|c|c|c|c|c|}
\hline \multirow[t]{2}{*}{$\begin{array}{l}\text { (Klinkesorn } \\
\text { \& Julian } \\
\text { McClement } \\
\text { s 2010) }\end{array}$} & lec & C & $\begin{array}{l}\mathrm{p} \\
(0.1 \\
-0 . \\
5 \%)\end{array}$ & & $5 \%$ tuna & $\begin{array}{l}\text { 1h pH 7.0; } 1 \text { hH } 2.0 ; 2 \\
\text { h pH 5.3; pH } 7.52 \text { h, } 2 \\
\text { mg/ml lipase (also } \\
\text { lower conc tried), } 5.5 \\
\text { mM bile salts (also } \\
2.5-6.5 \text { tried) }->\text { final }\end{array}$ & $\begin{array}{l}-/ 120 ; 2.3 \\
\mathrm{mmol} / \mathrm{ml}\end{array}$ & $\begin{array}{l}-/ 120 ; \underline{\mathbf{0 . 6}} \\
\underline{\mathbf{m m o l} / \mathbf{m l}}\end{array}$ & $\begin{array}{ll}0.1 \%: & - \\
/ 120 ; 1.9 & \\
\mathrm{mmol} / \mathrm{ml} & - \\
0.5 \%: & - \\
/ 120 ; 2.4 & \\
\mathrm{mmol} / \mathrm{ml} & \\
\end{array}$ & & \multirow{2}{*}{$\begin{array}{l}\text { A second layer clearly } \\
\text { lowers the lipolysis, but this } \\
\text { effect was gone by adding a } \\
\text { third pec layer and less } \\
\text { clear by adding a md third } \\
\text { layer. Increasing [bile salt] } \\
\text { or [lipase] resulted in a } \\
\text { higher final FFA content. }\end{array}$} \\
\hline & lec & C & $\begin{array}{l}\text { md } \\
(0.1 \\
-0 . \\
5 \%)\end{array}$ & & & FFA content measured & & & $\begin{array}{l}0.1 \%: \\
/ 120 ; 1.4 \\
\mathrm{mmol} / \mathrm{ml} \\
0.5 \%: \\
/ 120 ; 1.4 \\
\mathrm{mmol} / \mathrm{ml}\end{array}$ & & \\
\hline \multirow[t]{2}{*}{$\begin{array}{l}\text { (Lesmes et } \\
\text { al. 2010) }\end{array}$} & cas & If & & & $\begin{array}{l}10 \% \text { corn } \\
\text { oil }\end{array}$ & 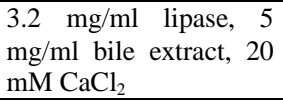 & $\underline{\mathbf{9}} / 50 ; 75$ & $6 / 50 ; 85$ & & & \multirow{2}{*}{$\begin{array}{l}\text { Adsorbing a second layer } \\
\text { slightly facilitated the } \\
\text { lipolysis, especially for cas- } \\
\text { lf. }\end{array}$} \\
\hline & If & cas & & & & & $5 / 50 ; 72$ & $5 / 50 ; 83$ & & & \\
\hline $\begin{array}{l}\text { (Mun et al. } \\
\text { 2006) }\end{array}$ & lec & C & $\mathrm{p}$ & & $\begin{array}{l}3 \% \text { corn } \\
\text { oil }\end{array}$ & $\begin{array}{l}\text { per ml } 1.6 \quad \mathrm{mg} \\
\text { pancreatic lipase and } 5 \\
\text { mg bile extract }\end{array}$ & $-/ 120 ; 250$ umol & $\begin{array}{l}-/ \quad 120 ; 38 \\
\text { umol }\end{array}$ & $\begin{array}{l}\text {-/ 120;, } 274 \\
\text { umol }\end{array}$ & & $\begin{array}{l}\text { The lec-c emulsion had a } \\
\text { much lower extent of } \\
\text { lipolysis compared to the } \\
\text { primary lec emulsion, but } \\
\text { also than the tertiary } \\
\text { emulsion. This bilayered } \\
\text { emulsion also showed more } \\
\text { extensive droplet } \\
\text { aggregation. }\end{array}$ \\
\hline $\begin{array}{l}\text { (McClemen } \\
\text { ts 2010) }\end{array}$ & $\mathrm{a}$ & C & $\mathrm{a}$ & $\begin{array}{l}\mathrm{c}- \\
\mathrm{a}- \\
\mathrm{c}- \\
\mathrm{a}\end{array}$ & - & & $5 / 100 ; 83$ & $3 / 100 ; 73$ & 4/ 100;81 & $\begin{array}{l}1 / 100 ; 82- \\
7 / 100 ; 55- \\
7 / 100 ; 36 \\
\underline{16 / 100 ; 13}\end{array}$ & $\begin{array}{l}\text { The lipolysis was lower for } \\
\text { emulsions with more } \\
\text { layers, especially above } 5 \\
\text { layers. }\end{array}$ \\
\hline
\end{tabular}

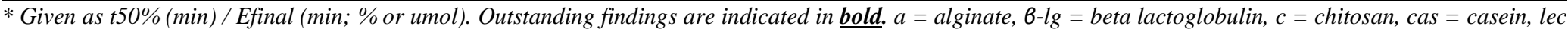

$=$ lecithin, If = lactoferrin, md = maltodextrin, $p=$ pectin, $T 20=$ Tween 


\subsection{REFERENCES}

Abbaspourrad, A., Datta, S.S., Weitz, D.A. (2013). Controlling Release From pH-Responsive Microcapsules. Langmuir. 29(41):12697-702.

Ahmed, K.., Li, Y., McClements, D.J., Xiao, H. (2012). Nanoemulsion- and emulsion-based delivery systems for curcumin: Encapsulation and release properties. Food Chemistry. 132(2):799-807.

Akkermans, C., van der Goot, AJ., Venema, P., van der Linden, E., Boom, R.M. (2008). Formation of fibrillar whey protein aggregates: Influence of heat and shear treatment, and resulting rheology. Food Hydrocolloids. 22(7):1315-25.

Albertsson, P.-A., Köhnke, R., Emek, S.C., Mei, J., Rehfeld, J.F., Akerlund, H.-E., Erlanson-Albertsson, C. (2007). Chloroplast membranes retard fat digestion and induce satiety: effect of biological membranes on pancreatic lipase/co-lipase. The Biochemical journal. 401(3):727-33.

Antipina, M.N., Sukhorukov, G.B. (2011). Remote control over guidance and release properties of composite polyelectrolyte based capsules. Advanced drug delivery reviews. 63(9):716-29.

Atuma, C., Strugala, V., Allen, A., Holm, L. (2001). The adherent gastrointestinal mucus gel layer: thickness and physical state in vivo. American journal of physiology and Gastrointestinal and liver physiology. 280(5):922-29.

Augustin, M.A., Hemar, Y. (2009). Nano- and micro-structured assemblies for encapsulation of food ingredients. Chemical Society Reviews. 38(4):902-12.

Augustin, M.A., Sanguansri, L., Rusli, J.K., Shen, Z., Cheng, L.J., Keogh, J., Clifton, P. (2014). Digestion of microencapsulated oil powders: in vitro lipolysis and in vivo absorption from a food matrix. Food \& function. 5(11):2905-12.

Bakala N'Goma, J.-C., Amara, S., Dridi, K., Jannin, V., Carrière, F. (2012). Understanding the lipid-digestion processes in the GI tract before designing lipid-based drug-delivery systems. Therapeutic delivery. 3(1):105-24.

Barbé, F., Ménard, O., Le Gouar, Y., Buffière, C., Famelart, M.-H., Laroche, B., Le Feunteun, S., et al. (2013). The heat treatment and the gelation are strong determinants of the kinetics of milk proteins digestion and of the peripheral availability of amino acids. Food Chemistry. 136:1203-12.

Batterham, R.L., Cowley, M A., Small, C.J., Herzog, H., Cohen, M.A., Dakin, C.L., Wren, A.M., et al. (2002). Gut hormone PYY(3-36) physiologically inhibits food intake. Nature. 418:650-54.

Bédard, M.F., de Geest, B.G., Skirtach, A.G., Möhwald, H., Sukhorukov, G.B. (2010). Polymeric microcapsules with light responsive properties for encapsulation and release. Advances in colloid and interface science. 158:2-14.

Beindorff,C.M., Ghislain, C.Y., Sergey, L., Melnikov, M., Winter, I. (2007). Satiety enhancing food products. Patent WO 2007115899 A1: 1-23.

Benshitrit, R.C., Levi, C.S., Tal, S.L., Shimoni, E., Lesmes, U. (2012). Development of oral food-grade delivery systems: current knowledge and future challenges. Food \& function. 3(1):10-21.

Berton-Carabin, C.C., Coupland, J.N., Elias, R.J. (2013). Effect of the lipophilicity of model ingredients on their location and reactivity in emulsions and solid lipid nanoparticles. Colloids and Surfaces A: Physicochemical and Engineering Aspects. 431:9-17.

Berton-Carabin, C.C., Ropers, M.-H., Genot, C. (2014). Lipid Oxidation in Oil-in-Water Emulsions: Involvement of the Interfacial Layer. Comprehensive Reviews in Food Science and Food Safety. 13(5):945-77.

Berton-carabin, C.C., Schroën, K. (2014). Pickering Emulsions for Food Applications : Background, Trends and Challenges. Annual Review in Food Science and Technology. 31(0):1-55.

Bialek, J.M., Melnikov, S.M., Winter, I. (2006). Satiety emulsions and food compositions. Patent US 2006/0105093 A1.

Binks, B.P. (2002). Particles as surfactants - similarit.ies and differences. Current Opinion in Colloid \& Interface Science. 7:21-41.

Blundell, J.E., Bellisle, F. (2013). Satiation, Satiety and the Control of Food Intake. Woodhead Publishing Limited, Cambridge, Philadelphia, New Delhi.

Bonnaire, L., Sandra, S., Helgason, T., Decker, E.A., Weiss, J., McClements, D.J. (2008). Influence of lipid physical state on the in vitro digestibility of emulsified lipids. Journal of agricultural and food chemistry. 56(10):3791-97.

Borel, T., Sabliov, C.M. (2014). Nanodelivery of bioactive components for food applications: Types of delivery systems, properties, and their effect on ADME profiles and toxicity of nanoparticles. Annual Review of Food Science and Technology. 5:1-17. 
Bornhorst, G.M., Singh, R.P. (2014). Gastric digestion in vivo and in vitro: How the structural aspects of food influence the digestive process. Annual Review of Food Science and Technology. 5:1-22.

Burton, D.D., Kim, H.J., Camilleri, M., Stephens, D.A., Mullan, B.P., O’Connor, M.K., \& Talley, N.J. (2005). Relationship of gastric emptying and volume changes after a solid meal in humans. Am $J$ Physiol Gastrointest Liver Physiol, 289(2): 261-6.

Butstraen, C., Salaün, F. (2014). Preparation of microcapsules by complex coacervation of gum Arabic and chitosan. Carbohydrate polymers. 99:608-16.

Calbet, J.A.L., MacLean, D.A. (1997). Role of caloric content on gastric emptying in humans. The Journal of physiology. 498(2):553-59.

Carriere, F., Barrowman, J.A., Verger, R., Laugier, R. (1993). Secretion and contribution to lipolysis of gastric and pancreatic lipases during a test meal in humans. Gastroenterology. 105(3):876-88.

Cerqueira, M.A., Pinheiro, A.C., Silva, H.D., Ramos, P.E., Azevedo, M.A., Flores-López, M.L., Rivera, M.C., et al. (2013). Design of Bio-nanosystems for Oral Delivery of Functional Compounds. Food Engineering Reviews. 6(1-2):1-19.

Chaprenet, J., Berton-Carabin, C.C., Elias, R.J., Coupland, J.N. (2014). Effect of interfacial properties on the reactivity of a lipophilic ingredient in multilayered emulsions. Food Hydrocolloids. 42:56-65.

Chen, P.W., Cadisch, G., Studart, A.R. (2014). Encapsulation of Aliphatic Amines Using Micro fluidics. Langmuir. 30:2346-50.

Cho, H.T., Salvia-Trujillo, L., Kim, J., Park, Y., Xiao, H., McClements, D.J. (2014). Droplet size and composition of nutraceutical nanoemulsions influences bioavailability of long chain fatty acids and Coenzyme Q10. Food Chemistry. 156:117-22.

Choi, S.-W., Zhang, Y., Xia, Y. (2010). A temperature-sensitive drug release system based on phase-change materials. Angewandte Chemie International Edition. 49(43):7904-8.

Christophersen, P.C., Christiansen, M.L., Holm, R., Kristensen, J., Jacobsen, J., Abrahamsson, B., Müllertz, A. (2014). Fed and fasted state gastro-intestinal in vitro lipolysis: In vitro in vivo relations of a conventional tablet, a SNEDDS and a solidified SNEDDS. European journal of pharmaceutical sciences. 57:232-39.

Chu, B.-S., Gunning, A.P., Rich, G.T., Ridout, M.J., Faulks, R.M., Wickham, M.S.J., Morris, V.J., et al. (2010). Adsorption of bile salts and pancreatic colipase and lipase onto digalactosyldiacylglycerol and dipalmitoylphosphatidylcholine monolayers. Langmuir. 26(12):9782-93.

Chu, B.-S., Rich, G.T., Ridout, M.J., Faulks, R.M., Wickham, M.S.J., Wilde, P.J. (2009). Modulating pancreatic lipase activity with galactolipids: effects of emulsion interfacial composition. Langmuir. 25(16):9352-60.

Cilla, A., Alegría, A., De Ancos, B., Sánchez-Moreno, C., Cano, M.P., Plaza, L., Clemente, G., et al. (2012). Bioaccessibility of Tocopherols, Carotenoids, and Ascorbic Acid from Milk- and Soy-Based Fruit Beverages: Influence of Food Matrix and Processing. Journal of agricultural and food chemistry. 60:7282-90.

Cook, M.T., Tzortzis, G., Charalampopoulos, D., Khutoryanskiy, V.V. (2012). Microencapsulation of probiotics for gastrointestinal delivery. Journal of controlled release. 162(1):56-67.

Coupe, A.J., Davis, S.S., Wilding, I.R. (1991). Variation in Gastrointestinal Transit of Pharmaceutical Dosage Forms in Healthy Subjects. Pharmaceutical research. 8(3):360.

Day, L., Golding, M., Xu, M., Keogh, J., Clifton, P., Wooster, T.J. (2014). Tailoring the digestion of structured emulsions using mixed monoglyceride-caseinate interfaces. Food Hydrocolloids. 36:15161.

De Boever, P., Deplancke, B., Verstraete, W. (2000). Fermentation by gut microbiota cultured in a simulator of the human intestinal microbial ecosystem is improved by supplementing a soygerm powder. The Journal of nutrition. 130(10):2599-2606.

Deligöz, H., Tieke, B. (2014). QCM-D study of layer-by-layer assembly of polyelectrolyte blend films and their drug loading-release behavior. Colloids and Surfaces A. 441:725-36.

Diepvens, K., Soenen, S., Steijns, J., Arnold, M., Westerterp-Plantenga, M. (2007). Long-term effects of consumption of a novel fat emulsion in relation to body-weight management. International journal of obesity. 31(6):942-49.

Dong, Q.-Y., Chen, M.-Y., Xin, Y., Qin, X.-Y., Cheng, Z., Shi, L.-E., Tang, Z.-X. (2013). Alginate-based and protein-based materials for probiotics encapsulation: a review. International Journal of Food Science \& Technology. 48(7):1339-51.

Duffy, N., Blonk, H.C.G., Beindorff, C.M., Cazade. M., Bot. A., Duchateau, G.S.M.J.E. (2009). OrganogelBased Emulsion Systems, Micro-Structural Features and Impact on In vitro Digestion. Journal of the American Oil Chemists’ Society. 86(8):733-41. 
EFSA. (2010). Scientific Opinion on the safety of anionic methacrylate copolymer for the proposed uses as a food additive. EFSA Journal. 8(7):1-1656.

EFSA. (2014). Regulated food ingredients applications. European Food Safety Authority. www.efsa.europa.eu/en/applicationshelpdesk/foodingredients.htm.

Elgart, A., Cherniakov, I., Aldouby, Y., Domb, A.J., Hoffman, A. (2012). Lipospheres and pro-nano lipospheres for delivery of poorly water soluble compounds. Chemistry and physics of lipids. 165(4):438-53.

Emin, M.A., Mayer-Miebach, E., Schuchmann, H.P. (2012). Retention of $\beta$-carotene as a model substance for lipophilic phytochemicals during extrusion cooking. LWT - Food Science and Technology. 48(2):302-7.

FDA. (2014). Ingredients, Packaging \& Labeling. U.S. Food and Drug Administration. www.fda.gov/Food/IngredientsPackagingLabeling/.

Fiore, A., Troise, A.D., Mogol, A., Roullier, V., Gourdon, A. (2012). Controlling the Maillard Reaction by Reactant Encapsulation: Sodium Chloride in Cookies. Journal of agricultural and food chemistry. 60:10808-14.

Firoozmand, H., Rousseau, D. (2014). Tailoring the morphology and rheology of phase-separated biopolymer gels using microbial cells as structure modifiers. Food Hydrocolloids. 42:204-14.

Gallier, S., Singh, H. (2012). Behavior of almond oil bodies during in vitro gastric and intestinal digestion. Food \& Function. 3(5):547-55.

Gallier, S., Tate, H., Singh, H. (2013). In vitro gastric and intestinal digestion of a walnut oil body dispersion. Journal of Agricultural and Food Chemistry. 61(2):410-17.

Garcia, C., Antona, C., Robert, B., Lopez, C., Armand, M. (2014). The size and interfacial composition of milk fat globules are key factors controlling triglycerides bioavailability in simulated human gastroduodenal digestion. Food Hydrocolloids. 35:494-504.

Garti, N., Hoshen, G., Aserin, A. (2012). Lipolysis and structure controlled drug release from reversed hexagonal mesophase. Colloids and surfaces B: Biointerfaces. 94:36-43.

Geraedts, M.C.P., Troost, F.J., Munsters, M.J.M., Stegen, J.H.C.H., de Ridder, R.J., Conchillo, J.M., Kruimel, J.W., et al. (2011). Intraduodenal administration of intact pea protein effectively reduces food intake in both lean and obese male subjects. PLOS ONE. 6(9):1-7.

Gharsallaoui, A., Roudaut, G., Chambin, O., Voilley, A., Saurel, R. (2007). Applications of spray-drying in microencapsulation of food ingredients: An overview. Food Research International. 40(9):1107-21.

Giang, T.M., Le Feunteun, S., Gaucel, S., Brestaz, P., Anton, M., Meynier, A., Trelea, I.C. (2015). Dynamic modeling highlights the major impact of droplet coalescence on the in vitro digestion kinetics of a whey protein stabilized submicron emulsion. Food Hydrocolloids. 43:66-72.

Gibbs, B.F., Kermasha, S., Alli, I., Mulligan, C.N. (1999). Encapsulation in the food industry: a review. International journal of food sciences \& nutrition. 50(3):213-24.

Gibbs, J., Smith, G.P. (1982). Gut peptides and food in the gut produce similar satiety effects. Peptides. 3(3):553-57.

Golding, M., Wooster, T.J., Day, L., Xu, M., Lundin, L., Keogh, J., Clifton, P. (2011). Impact of gastric structuring on the lipolysis of emulsified lipids. Soft Matter. 7(7):3513.

Golding, M.D., de Groot, P.W.N., Koppert, R.J., Melnikov, S.M., Pelan, E.G. (2009). Satiety emulsions and food compositions. Patent US 2009/0317509 A1: 1-7.

Grabovac, V., Guggi, D., Bernkop-Schnürch, A. (2005). Comparison of the mucoadhesive properties of various polymers. Advanced drug delivery reviews. 57(11):1713-23.

Granger, C., Barey, P., Toutain, J., Cansell, M. (2005). Direct quantification of protein partitioning in oil-inwater emulsion by front-face fluorescence: avoiding the need for centrifugation. Colloids and surfaces B: Biointerfaces. 43(3-4):158-62.

Gun, W.J., Routh, A.F. (2013). Formation and Characterization of pH-Responsive Liquid Core Microcapsules. Langmuir. 29(40):12541-48.

Gunaseelan, K., Romsted, L.S., Gallego, M.-J.P., González-Romero, E., Bravo-Díaz, C. (2006). Determining alpha-tocopherol distributions between the oil, water, and interfacial regions of macroemulsions: novel applications of electroanalytical chemistry and the pseudophase kinetic model. Advances in colloid and interface science. 123-126:303-11.

Gupta, R., Rousseau, D. (2012). Surface-active solid lipid nanoparticles as Pickering stabilizers for oil-inwater emulsions. Food \& function. 3(3):302-11.

Guzey, D., McClements, D.J. (2006). Formation, stability and properties of multilayer emulsions for application in the food industry. Advances in colloid and interface science. 128(130):227-48.

Herslof, B., Lindmark, L., Bohlinder, K., Carlsson, A. (2003). Satiety Products. Patent US 6,517,883 B1. 
Hoebler, C., Guillon, F., Fardet, A., Cherbut, C., \& Barry, J. (1998). Gastrointestinal or Simulated In vitro Digestion Changes Dietary Fibre Properties and their Fermentation. Journal of the Science of Food and Agriculture, 77: 327-33.

Horn, A.F., Barouh, N., Nielsen, N.S., Baron, C.P., Jacobsen, C. (2013). Homogenization pressure and temperature affect protein partitioning and oxidative stability of emulsions. Journal of the American Oil Chemists' Society. 90(10):1541-50.

Hu, B., Zhang, L., Liang, R., Chen, F., He, L., Hu, B., Zeng, X. (2015). Cross-linking of Interfacial Casein Layer with Genipin Prevented pH Induced Structural Instability and Lipase Digestibility of the Fat Droplets. Journal of agricultural and food chemistry. 63(7):2033-40.

Hu, M., Li, Y., Decker, E.A., McClements, D.J. (2010)(a). Role of calcium and calcium-binding agents on the lipase digestibility of emulsified lipids using an in vitro digestion model. Food Hydrocolloids. 24(8):719-25.

Hu, M., Li, Y., Decker, E.A., Xiao, H., McClements, D.J. (2010)(b). Impact of Layer Structure on Physical Stability and Lipase Digestibility of Lipid Droplets Coated by Biopolymer Nanolaminated Coatings. Food Biophysics. 6:37-48.

Hur, S.J., Decker, E.A., McClements, D.J. (2009). Influence of initial emulsifier type on microstructural changes occurring in emulsified lipids during in vitro digestion. Food Chemistry. 114:253-62.

Hur, S.J., Lim, B.O., Decker, E,A., McClements, D.J. (2011). In vitro human digestion models for food applications. Food Chemistry. 125:1-12.

Ibekwe, V.C., Fadda, H.M., McConnell, E.L., Khela, M.K., Evans, D.F., Basit, A.W. (2008). Interplay between intestinal $\mathrm{pH}$, transit time and feed status on the in vivo performance of $\mathrm{pH}$ responsive ileocolonic release systems. Pharmaceutical research. 25(8):1828-35.

Ibekwe, V.C., Fadda, H.M., Parsons, G.E., Basit, A.W. (2006). A comparative in vitro assessment of the drug release performance of $\mathrm{pH}$-responsive polymers for ileo-colonic delivery. International journal of pharmaceutics. 308(1-2):52-60.

Inui, A., Asakawa, A., Bowers, C.Y., Mantovani, G., Laviano, A., Meguid, M.M., Fujimiya, M. (2004). Ghrelin, appetite, and gastric motility: the emerging role of the stomach as an endocrine organ. FASEB journal: official publication of the Federation of American Societies for Experimental Biology. 18(3):439-56.

Israeli-Lev, G., Livney, Y.D. (2014). Self-assembly of hydrophobin and its co-assembly with hydrophobic nutraceuticals in aqueous solutions: Towards application as delivery systems. Food Hydrocolloids. 35:28-35.

Jódar-Reyes, A.B., Torcello-Gómez, A., Wulff-Pérez, M., Gálvez-Ruiz, M.J., Martín-Rodríguez, A. (2010). Different stability regimes of oil-in-water emulsions in the presence of bile salts. Food Research International. 43(6):1634-41.

Joyce, P., Tan, A., Whitby, C.P., Prestidge, C. A. (2014). The Role of Porous Nanostructure in Controlling Lipase-Mediated Digestion of Lipid Loaded into Silica Particles. Langmuir. 30:2779-88.

Joye, I.J., McClements, D.J. (2013). Production of nanoparticles by anti-solvent precipitation for use in food systems. Trends in Food Science \& Technology. 34(2):109-23.

Kenmogne-Domguia, H.B. (2012). PhD thesis entitled Évolutions structurales et oxydation des lipides lors de la digestion in vitro d'émulsions modèles. INRA, UR1268 Biopolymères Interactions et Assemblages, F-44316 Nantes, France.

Keogh, J.B., Wooster, T.J., Golding, M., Day, L., Clifton, P.M. (2011). Slowly and Rapidly Digested Fat Emulsions Are Equally Satiating but Their Triglycerides Are Differentially Absorbed and Metabolized. The Journal of nutrition. 809-15.

Klinkesorn, U., McClements, D.J. (2010). Impact of Lipase, Bile Salts, and Polysaccharides on Properties and Digestibility of Tuna Oil Multilayer Emulsions Stabilized by Lecithin-Chitosan. Food Biophysics. 5(2):73-81.

Knutson, L., Fridblom, H., Viberg, A., Sein, A., Lennerna, H. (2010). Gastrointestinal metabolism of a vegetable-oil emulsion in healthy. American Journal of Clinical Nutrition. 92:515-24.

Kong, F., Singh, R.P. (2010). A human gastric simulator (HGS) to study food digestion in human stomach. Journal of food science. 75(9):E627-35.

Kozu, H., Kobayashi, I., Neves, M.A., Nakajima, M., Uemura, K., Sato, S., Ichikawa, S. (2014). PIV and CFD studies on analyzing intragastric flow phenomena induced by peristalsis using a human gastric flow simulator. Food \& function. 5(8):1839-47.

Krishnamachari, Y., Madan, P., Lin, S. (2007). Development of pH- and time-dependent oral microparticles to optimize budesonide delivery to ileum and colon. International journal of pharmaceutics. 338:23847. 
Kroes-Nijboer, A., Venema, P., van der Linden, E. (2012). Fibrillar structures in food. Food \& function. 3(3):221-27.

Kunz, P., Feinle-Bisset, C., Faas, H., Boesiger, P., Fried, M., Steingötter, A., Schwizer, W. (2005). Effect of ingestion order of the fat component of a solid meal on intragastric fat distribution and gastric emptying assessed by MRI. Journal of magnetic resonance imaging. 21(4):383-90.

Lam, P.L., Gambari, R. (2014). Advanced progress of microencapsulation technologies: In vivo and in vitro models for studying oral and transdermal drug deliveries. Journal of controlled release. 178:25-45.

Laouini, A., Koutroumanis, K.P., Charcosset, C., Georgiadou, S., Fessi, H., Holdich, R.G., Vladisavljević, G.T. (2013). pH-Sensitive Micelles for Targeted Drug Delivery Prepared Using a Novel Membrane Contactor Method. Applied Materials \& Interfaces. 5(18):8939-47.

Lavin, J.H., Wittert, G.A., Andrews, J., Yeap, B., Wishart, J.M., Morris, H.A., Morley, J.E., et al. (1998). Interaction of insulin, glucagon-like peptide 1 , gastric inhibitory polypeptide, and appetite in response to intraduodenal carbohydrate. American Journal of Clinical Nutrition. 68:591-98.

Leal-Calderon, F., Schmitt, V., Bibette, J. (2007). Emulsion Science, Basic Principles. NY: Springer Science.

Lesmes, U., Baudot, P., McClements, D.J. (2010). Impact of interfacial composition on physical stability and in vitro lipase digestibility of triacylglycerol oil droplets coated with lactoferrin and/or caseinate. Journal of agricultural and food chemistry. 58(13):7962-69.

Lesmes, U., McClements, D.J. (2009). Structure-function relationships to guide rational design and fabrication of particulate food delivery systems. Trends in Food Science \& Technology. 20(10):44857.

Li, X., Fang, Y., Phillips, G.O., Al-Assaf, S. (2013). Improved Sugar Beet Pectin-Stabilized Emulsions through Complexation with Sodium Caseinate. Journal of agricultural and food chemistry. 61(6):1388-96.

Li, Y., Hu, M., McClements, D.J. (2011). Factors affecting lipase digestibility of emulsified lipids using an in vitro digestion model: Proposal for a standardised pH-stat method. Food Chemistry. 126(2):498505.

Li, Y., Hu, M., Xiao, H., Du, Y., Decker, E.A., McClements, D.J. (2010). Controlling the functional performance of emulsion-based delivery systems using multi-component biopolymer coatings. European journal of pharmaceutics and biopharmaceutics. 76(1):38-47.

Li, Y., Kim, J., Park, Y., McClements, D.J. (2012). Modulation of lipid digestibility using structured emulsion-based delivery systems: comparison of in vivo and in vitro measurements. Food \& function. 3(5):528-36.

Li, Y., McClements, D.J. (2014)(a). Modulating lipid droplet intestinal lipolysis by electrostatic complexation with anionic polysaccharides: Influence of cosurfactants. Food Hydrocolloids. 35:367-74.

Li, Y., McClements, D.J. (2014)(b). Influence of cosurfactant on the behavior of structured emulsions under simulated intestinal lipolysis conditions. Food Hydrocolloids. 40:96-103.

Liu, F., Tang, C.-H. (2014). Phytosterol colloidal particles as Pickering stabilizers for emulsions. Journal of agricultural and food chemistry. 62(22):5133-41.

Losada-Barreiro, S., Bravo-Diaz, C., Paiva-Martins, F., Romsted, L.S. (2013). Maxima in Antioxidant Distributions and Efficiencies with Increasing Hydrophobicity of Gallic Acid and Its Alkyl Esters. The Pseudophase Model Interpretation of the "Cut off Effect." Journal of agricultural and food chemistry. 61:6533-43.

Luo, Q., Boom, R.M., Janssen, A.E.M. (2015). Digestion of protein and protein gels in simulated gastric environment. LWT - Food Science and Technology. 63(1):161-8.

Luo, R., Venkatraman, S.S., Neu, B. (2013). Layer-by-Layer Polyelectrolyte - Polyester Hybrid Microcapsules for Encapsulation and Delivery of Hydrophobic Drugs. Biomacromolecules. 14:226271.

Mackie, A., Gunning, A., Wilde, P., Morris, V. (1999). Orogenic Displacement of Protein from the Air/Water Interface by Competitive Adsorption. Journal of colloid and interface science. 210(1):157-66.

Madene, A., Jacquot, M., Scher, J., Desobry, S. (2006). Flavour encapsulation and controlled release - a review. International Journal of Food Science \& Technology. 41(1):1-21.

Makkhun, S., Khosla, A., Foster, T., McClements, D.J., Grundy, M.M.L., Gray, D.A. (2015). Impact of extraneous proteins on the gastrointestinal fate of sunflower seed (Helianthus annuus) oil bodies: a simulated gastrointestinal tract study. Food \& function. 6(1):124-33.

Malaki Nik, A., Wright, A.J., Corredig, M. (2011). Impact of interfacial composition on emulsion digestion and rate of lipid hydrolysis using different in vitro digestion models. Colloids and surfaces B: Biointerfaces. 83(2):321-30. 
Maldonado-Valderrama, J., Wilde, P., Macierzanka, A., Mackie, A. (2011). The role of bile salts in digestion. Advances in colloid and interface science. 165:36-46.

Malinauskytė, E., Ramanauskaitė, J., Leskauskaite, D., Devold, T.G., Schüller, R.B., Vegarud, G.E. (2014). Effect of human and simulated gastric juices on the digestion of whey proteins and carboxymethylcellulose-stabilised O/W emulsions. Food Chemistry. 165:104-12.

Maljaars, J., Peters, H.P.F., Masclee, A.M. (2007). Review article: The gastrointestinal tract: neuroendocrine regulation of satiety and food intake. Alimentary Pharmacology \& Therapeutics. 26(2):241-50.

Maljaars, P.W.J., Peters, H.P.F., Kodde, A., Geraedts, M., Troost, F.J., Haddeman, E., Masclee, A.A.M. (2011). Length and site of the small intestine exposed to fat influences hunger and food intake. The British journal of nutrition. 106(10):1609-15.

Maljaars, P.W.J., Peters, H.P.F., Mela, D.J., Masclee, A.A.M. (2008)(a). Ileal brake: a sensible food target for appetite control. A review. Physiology \& behavior. 95(3):271-81

Maljaars, P.W.J., Romeyn, E.A., Haddeman, E., Peters, H.P.F., Masclee, A.A.M. (2009). Effect of fat saturation on satiety, hormone release, and food intake. The American Journal of Clinical Nutrition. 89:1019-24.

Maljaars, P.W.J., Symersky, T., Kee, B.C., Haddeman, E., Peters, H.P.F., Masclee, A.A.M. (2008)(b). Effect of ileal fat perfusion on satiety and hormone release in healthy volunteers. International journal of obesity. 32(11):1633-39.

Mantovani, R.A., Cavallieri, A.L.F., Netto, F.M., Cunha, R.L. (2013). Stability and in vitro digestibility of emulsions containing lecithin and whey proteins. Food \& function. 4(9):1322-31.

Marciani, L., Gowland, P.A., Fillery-Travis, A., Manoj, P., Wright, J., Smith, A., Young, P., et al. (2001). Assessment of antral grinding of a model solid meal with echo-planar imaging. American journal of physiology. Gastrointestinal and liver physiology. 280(5):G844-49.

Marieb, E.N. (1999). Anatomie et physiologie humaines. Québec, Canada: De Boeck, pp. 1-1194.

Marze, S. (2013). Bioaccessibility of nutrients and micronutrients from dispersed food systems: impact of the multiscale bulk and interfacial structures. Critical Reviews in Food Science and Nutrition. 53(1):76108.

Marze, S., Algaba, H., Marquis, M. (2014). A microfluidic device to study the digestion of trapped lipid droplets. Food \& function. 5(7):1481-88.

Marze, S., Choimet, M., Foucat, L. (2012). In vitro digestion of emulsions: mechanistic and experimental models. Soft Matter. 8(42):10982-93.

Matalanis, A., McClements, D.J. (2013). Hydrogel microspheres for encapsulation of lipophilic components: Optimization of fabrication \& performance. Food Hydrocolloids. 31(1):15-25.

McClements, D.J. (2005). Food Emulsions: Principles, Practices, and Techniques. CRC Press. Second ed.

McClements, D.J. (2010). Design of nano-laminated coatings to control bioavailability of lipophilic food components. Journal of Food Science. 75(1):R30-42.

McClements, D.J., Decker, E.A., Park, Y. (2009). Controlling lipid bioavailability through physicochemical and structural approaches. Critical Reviews in Food Science and Nutrition. 49(1):48-67.

McClements, D.J., Decker, E.A., Weiss, J. (2007). Emulsion-based delivery systems for lipophilic bioactive components. Journal of food science. 72(8):109-24.

McClements, D.J., Li, Y. (2010)(a). Review of in vitro digestion models for rapid screening of emulsionbased systems. Food \& function. 1(1):32-59.

McClements, D.J., Li, Y. (2010)(b). Structured emulsion-based delivery systems: controlling the digestion and release of lipophilic food components. Advances in colloid and interface science. 159(2):213-28.

McConnell, E.L., Fadda, H.M., Basit, A.W. (2008). Gut instincts: explorations in intestinal physiology and drug delivery. International journal of pharmaceutics. 364(2):213-26.

Meshulam, D., Lesmes, U. (2013). Responsiveness of emulsions stabilized by lactoferrin nano-particles to simulated intestinal conditions. Food \& function. 5(1):65-73.

Minekus, M. (2015). The TNO Gastro-Intestinal Model (TIM). In: Verhoeckx K, Cotter P, López-Expósito I et al (eds) The impact of food bioactives on health: in vitro and ex vivo models. Springer International Publishing, New York, pp. 37-46.

Minekus, M., Alminger, M., Alvito, P., Ballance, S., Bohn, T., Bourlieu, C., Carrière, F., et al. (2014). A standardised static in vitro digestion method suitable for food - an international consensus. Food \& function. 5:1113-24.

Molly, K., van de Woestyne, M., de Smet, I., Verstraete, W. (1994). Validation of the Simulator of the Human Intestinal Microbial Ecosystems (SHIME) Reaction Using Microorganism-associated Activities. Microbial Ecology in Health and Disease. 7:191-200. 
Moreau, H., Sauniere, J.F., Gargouri, Y., Pieroni, G., Verger, R., Sarles, H. (1988). Human Gastric Lipase: Variations Induced by Gastrointestinal Hormones and by Pathology. Scandinavian Journal of Gastroenterology. 23(9):1044-48.

Moriguchi, I., Hirono, S., Liu, Q., Nakagome, I., Matsushita, Y. (1992). Simple method of calculating octanol/water partition coefficient. Chemical and Pharmaceutical Bulletin. 40(17):127-30.

Mun, S., Decker, E.A., McClements, D.J. (2007). Influence of emulsifier type on in vitro digestibility of lipid droplets by pancreatic lipase. Food Research International. 40(6):770-81.

Mun, S., Decker, E.A., Park, Y., Weiss, J., McClements, D.J. (2006). Influence of Interfacial Composition on in vitro Digestibility of Emulsified Lipids: Potential Mechanism for Chitosan's Ability to Inhibit Fat Digestion. Food Biophysics. 1(1):21-29.

Mun, S., Kim, Y.-R., Shin, M., McClements, D.J. (2015). Control of lipid digestion and nutraceutical bioaccessibility using starch-based filled hydrogels: Influence of starch and surfactant type. Food Hydrocolloids. 44:380-89.

Neubauer, M.P., Poehlmann, M., Fery, A. (2014). Microcapsule mechanics: From stability to function. Advances in colloid and interface science. 207:65-80.

Olsson, C., Holmgren, S. (2001). The control of gut motility. Comparative Biochemistry and Physiology Part A. 128(3):479-501.

Paques, J.P., van der Linden, E., van Rijn, C.J.M., Sagis, L.M.C. (2014). Preparation methods of alginate nanoparticles. Advances in colloid and interface science. 209:163-71.

Park, K.M., Sung, H., Choi, S.J., Choi, Y.J., Chang, P.-S. (2014). Double-layered microparticles with enzyme-triggered release for the targeted delivery of water-soluble bioactive compounds to small intestine. Food Chemistry. 161:53-59.

Patel, A.R., Drost, E., Seijen ten Hoorn, J., Velikov, K.P. (2013). Fabrication and characterization of emulsions with $\mathrm{pH}$ responsive switchable behavior. Soft Matter. 9(29):6747-51.

Pedersen-Bjergaard, U., Høt, U., Kelbæk, H., Schifter, S., Rehfeld, F.J., Faber, J., Christensen, N.J. (1996). Influence of meal composition on postprandial peripheral plasma concentrations of vasoactive peptides in man. Scandinavian Journal of Clinical \& Laboratory Investigation. 56(6):497-503.

Pickering, S.U. (1907). Emulsions. Journal of the Chemical Society. 91:2001-21.

Pilichiewicz, A.N., Chaikomin, R., Brennan, I.M., Wishart, J.M., Rayner, C.K., Jones, K.L., Smout, A.J.P.M., et al. (2007). Load-dependent effects of duodenal glucose on glycemia, gastrointestinal hormones, antropyloroduodenal motility, and energy intake in healthy men. American Journal of Physiology Endocrinol Metabolism. 293:743-53.

Porter, C.J., Charman, W.N. (2001). In vitro assessment of oral lipid based formulations. Advanced drug delivery reviews. 50:S127-47.

Rampon, V., Genot, C., Riaublanc, A., Anton, M., Axelos, M.A.V., McClements, D.J. (2003). Front-face fluorescence spectroscopy study of globular proteins in emulsions: displacement of BSA by a nonionic surfactant. Journal of agricultural and food chemistry. 51(9):2482-89.

Rampon, V., Lethuaut, L., Mouhous-Riou, N., Genot, C. (2001). Interface characterization and aging of bovine serum albumin stabilized oil-in-water emulsions as revealed by front-surface fluorescence. Journal of agricultural and food chemistry. 49(8):4046-51.

Rayner, M., Timgren, A., Sjöö, M., Dejmek, P. (2012). Quinoa starch granules: a candidate for stabilising food-grade Pickering emulsions. Journal of the science of food and agriculture. 92(9):1841-47.

Raynes, J.K., Carver, J.A., Gras, S.L., Gerrard, J.A. (2014). Protein nanostructures in food - Should we be worried? Trends in Food Science \& Technology. 37(1):42-50.

Reis, P., Holmberg, K., Miller, R., Krägel, J., Grigoriev, D.O., Leser, M.E., Watzke, H.J. (2008). Competition between lipases and monoglycerides at interfaces. Langmuir. 24(14):7400-7407.

Rossier-Miranda, F.J., Schroën, K., Boom, R. (2010). Mechanical characterization and pH response of fibrilreinforced microcapsules prepared by layer-by-layer adsorption. Langmuir. 26(24):19106-13.

Rossier-Miranda, F.J., Schroën, K., Boom, R. (2012). Microcapsule production by an hybrid colloidosomelayer-by-layer technique. Food Hydrocolloids. 27(1):119-25.

Rousseau, D. (2013). Trends in structuring edible emulsions with Pickering fat crystals. Current Opinion in Colloid \& Interface Science. 18(4):283-91.

Ruiz-Rodriguez, P.E., Meshulam, D., Lesmes, U. (2014). Characterization of Pickering O/W Emulsions Stabilized by Silica Nanoparticles and Their Responsiveness to In vitro Digestion Conditions. Food Biophysics. 4:406-15.

Russel, W.B., Saville, D.A., Schowalter, W.R. (1989). Colloidal Dispersions. Cambridge University Press, Cambridge, pp. 1-525. 
Ryan, A.T., Feinle-Bisset, C., Kallas, A., Wishart, J.M., Clifton, P.M., Horowitz, M., Luscombe-Marsh, N.D. (2012). Intraduodenal protein modulates antropyloroduodenal motility, hormone release, glycemia, appetite, and energy intake in lean men. American Journal of Clinical Nutrition. 96(7):474-82.

Sakr, O.S., Borchard, G. (2013). Encapsulation of Enzymes in Layer-by-Layer (LbL) Structures : Latest Advances and Applications. Biomacromolecules. 14:2117-35.

Salminen, H., Weiss, J. (2014). Electrostatic adsorption and stability of whey protein-pectin complexes on emulsion interfaces. Food Hydrocolloids. 35:410-19.

Santivarangkna, C., Kulozik, U., Foerst, P. (2007). Alternative drying processes for the industrial preservation of lactic acid starter cultures. Biotechnology progress. 23(2):302-15.

Sawalha, H., Fan, Y., Schroën, K., Boom, R. (2008). Preparation of hollow polylactide microcapsules through premix membrane emulsification-Effects of nonsolvent properties. Journal of Membrane Science. 325(2):665-71.

Sawalha, H., Schroën, K., Boom, R. (2009). Hollow Polylactide Microcapsules with Controlled Morphology and Thermal and Mechanical Properties. AIChE journal. 55(11):2827-34.

Sawalha, H., Schroën, K., Boom, R. (2011). Biodegradable polymeric microcapsules: Preparation and properties. Chemical Engineering Journal. 169(1-3):1-10.

Schönhoff, M. (2003). Layered polyelectrolyte complexes : physics of formation and molecular properties. Journal of Physics: Condensed matter. 15:1781-1808.

Schroën, K., Bliznyuk, O., Muijlwijk, K., Sahin, S., Berton-Carabin, C.C. (2015). Microfluidic emulsification devices: from micrometer insights to large-scale food emulsion production. Current Opinion in Food Science. 3:33-40.

Serfert, Y., Lamprecht, C., Tan, C.-P., Keppler, J.K., Appel, E., Rossier-Miranda, F.J., Schroën, K., et al. (2014). Characterisation and use of $\beta$-lactoglobulin fibrils for microencapsulation of lipophilic ingredients and oxidative stability thereof. Journal of Food Engineering. 143:53-61.

Shahidan, N., Liu, R., Thaiboonrod, S., Alexander, C., Shakesheff, K.M., Saunders, B.R. (2013). Hollow colloidosomes prepared using accelerated solvent evaporation. Langmuir. 29(45):13676-85.

Simon, G.L., Gorbach, S.L. (1986). The human intestinal microflora. Digestive Diseases and Sciences. 31(S9):147-62.

Simovic, S., Heard, P., Hui, H., Song, Y., Peddie, F., Davey, A.K., Lewis, A., et al. (2009). Dry Hybrid Lipid - Silica Microcapsules Engineered from Submicron Lipid Droplets and Nanoparticles as a Novel Delivery System for Poorly Soluble Drugs. Molecular pharmaceutics. 6(3):861-72.

Singh, H., Ye, A., Horne, D. (2009). Structuring food emulsions in the gastrointestinal tract to modify lipid digestion. Progress in lipid research. 48(2):92-100.

Situ, W., Chen, L., Wang, X., Li, X. (2014). Resistant starch film-coated microparticles for an oral colonspecific polypeptide delivery system and its release behaviors. Journal of agricultural and food chemistry. 62(16):3599-3609.

Sjöö, M., Emek, S.C., Hall, T., Rayner, M., Wahlgren, M. (2015). Barrier properties of heat treated starch Pickering emulsions. Journal of Colloid and Interface Science. 450:182-88.

Smit, H.J., Keenan, E., Kovacs, E.M.R., Wiseman, S.A., Peters, H.P.F., Mela, D.J., Rogers, P.J. (2011). No efficacy of processed Fabuless (Olibra) in suppressing appetite or food intake. European journal of clinical nutrition. 65(1):81-86.

Speranza, A., Corradini, M.G., Hartman, T.G., Ribnicky, D., Oren, A., Rogers, M.A. (2013). Influence of Emulsifier Structure on Lipid Bioaccessibility in Oil - Water Nanoemulsions. Journal of agricultural and food chemistry. 61:6505-15.

Strader, A.D., Vahl, T.P., Jandacek, R.J., Woods, S.C., D’Alessio, D.A., Seeley, R.J. (2005). Weight loss through ileal transposition is accompanied by increased ileal hormone secretion and synthesis in rats. American Journal of Physiology Endocrinol Metabolism. 288:447-53.

Tan, A., Colliat-dangus, P., Whitby, C.P., Prestidge, C.A. (2014). Controlling the Enzymatic Digestion of Lipids Using Hybrid Nanostructured Materials. Applied Materials \& Interfaces. 6:15363-71.

Tan, C.S., Jejurikar, A., Rai, B., Bostrom, T., Lawrie, G., Grøndahl, L. (2009). Encapsulation of a glycosaminoglycan in hydroxyapatite/alginate capsules. Journal of biomedical materials research Part A. 91(3):866-77.

Tikekar, R.V., Nitin, N. (2011). Effect of physical state (solid vs. liquid) of lipid core on the rate of transport of oxygen and free radicals in solid lipid nanoparticles and emulsion. Soft Matter. 7(18):8149.

Timgren, A., Rayner, M., Sjöö, M., Dejmek, P. (2011). Starch particles for food based Pickering emulsions. Procedia Food Science. 1:95-103. 
Tokle, T., Mao, Y., McClements, D.J. (2013). Potential biological fate of emulsion-based delivery systems: lipid particles nanolaminated with lactoferrin and $\beta$-lactoglobulin coatings. Pharmaceutical research. 30(12):3200-3213.

Torcello-Gómez, A., Jódar-Reyes, A.B., Maldonado-Valderrama, J., Martín-Rodríguez, A. (2012). Effect of emulsifier type against the action of bile salts at oil-water interfaces. Food Research International. 48(1):140-47.

Torcello-Gómez, A., Maldonado-Valderrama, J., Martín-Rodríguez, A., McClements, D.J. (2011). Physicochemical properties and digestibility of emulsified lipids in simulated intestinal fluids: influence of interfacial characteristics. Soft Matter. 7(13):6167.

Turchiuli, C., Jimenez Munguia, M.T., Hernandez Sanchez, M., Cortes Ferre, H., Dumoulin, E. (2014). Use of different supports for oil encapsulation in powder by spray drying. Powder Technology. 255:1038.

Turton, M.D., O’Shea, D., Gunn, I., Beak, S.A., Edwards, C.M., Meeran, K., Choi, S.J., et al. (1996). A role for glucagon-like peptide-1 in the central regulation of feeding. Nature. 379(4):69-72.

Tzoumaki, M.V., Moschakis, T., Scholten, E., Biliaderis, C.G. (2013). In vitro lipid digestion of chitin nanocrystal stabilized o/w emulsions. Food \& function. 4(1):121-29.

van Aken, G.A. (2010). Relating Food Emulsion Structure and Composition to the Way It Is Processed in the Gastrointestinal Tract and Physiological Responses: What Are the Opportunities? Food Biophysics. 5(4):258-83.

van Aken, G.A., Vingerhoeds MH., de Hoog EHA. (2007). Food colloids under oral conditions. Current Opinion in Colloid \& Interface Science. 12(4-5):251-62.

van Avesaat, M., Troost, F.J., Ripken, D., Hendriks, H.F., Masclee, A.A.M. (2015). Ileal brake activation: macronutrient specific effects on eating behavior? International journal of obesity. 39:235-43.

van Klitzing, R. (2006). Internal structure of polyelectrolyte multilayer assemblies. Physical chemistry chemical physics. 8(43):5012-33.

Vaughan, R.W., Bauer, S., Wise, L. (1973). Volume and pH of gastric juice in Obese Patients. Anesthesiology. 43(6):686-89.

Vingerhoeds, M.H., Blijdenstein, T.B.J., Zoet, FD., van Aken, G.A. (2005). Emulsion flocculation induced by saliva and mucin. Food Hydrocolloids. 19(5):915-22.

Vitaglione, P., Barone Lumaga, R., Ferracane, R., Radetsky, I., Mennella, I., Schettino, R., Koder, S., et al. (2012). Curcumin bioavailability from enriched bread: the effect of microencapsulated ingredients. Journal of agricultural and food chemistry. 60(13):3357-66.

Walstra, P. (2003). Physical Chemistry of Foods. Marcel Dekker Inc. New York, pp. 1-832.

Wang, R., Fu, Y., Lai, L. (1997). A New Atom-Additive Method for Calculating Partition Coefficients. Journal of Chemistry and Inf. Computer Science. 37:615-21.

Waninge, R., Walstra, P., Bastiaans, J., Nieuwenhuijse, H., Nylander, T., Paulsson, M., Bergenståhl, B. (2005). Competitive adsorption between beta-casein or beta-lactoglobulin and model milk membrane lipids at oil-water interfaces. Journal of agricultural and food chemistry. 53(3):716-24.

WHO. (2014). Obesity and overweight. World Health Organization Media centre, Fact sheet N311. www.who.int/mediacentre/factsheets/fs311/en/.

Wickham, M.J.S., Faulks, R.M., Mann, J., Mandalari, G. (2012). The Design, Operation, and Application of a Dynamic Gastric Model. Dissolution Technologies. 19(3):15-22.

Wilcox, M.D., Brownlee, I.A., Richardson, J.C., Dettmar, P.W., Pearson, J.P. (2014). The modulation of pancreatic lipase activity by alginates. Food Chemistry. 146:479-84.

Wrenn, S.P., Dicker, S.M., Small, E.F., Dan, N.R., Mleczko, M., Schmitz, G., Lewin, P.A. (2012). Bursting bubbles and bilayers. Theranostics. 2(12):1140-59.

Wu, Z.M., Zhou, L., Guo, X.D., Jiang, W., Ling, L., Qian, Y., Luo, K.Q., et al. (2012). HP55-coated capsule containing PLGA/RS nanoparticles for oral delivery of insulin. International journal of pharmaceutics. 425(1-2):1-8.

Xiao, Z., Liu, W., Zhu, G., Zhou, R., Niu, Y. (2014). A review of the preparation and application of flavour and essential oils microcapsules based on complex coacervation technology. Journal of the science of food and agriculture. 94(8):1482-94.

Xu, D., Wang, X., Jiang, J., Yuan, F., Gao, Y. (2012). Impact of whey protein - Beet pectin conjugation on the physicochemical stability of $\beta$-carotene emulsions. Food Hydrocolloids. 28(2):258-66.

Xu, D., Yuan, F., Gao, Y., Panya, A., McClements, D.J., Decker, E.A. (2014). Influence of whey proteinbeet pectin conjugate on the properties and digestibility of $\beta$-carotene emulsion during in vitro digestion. Food Chemistry. 156:374-79. 
Yan, M., Liu, B., Jiao, X., Qin, S. (2014). Preparation of phycocyanin microcapsules and its properties. Food and Bioproducts Processing. 92:89-97.

Yang, D., Wang, X.-Y., Ji, C.-M., Lee, K.-T., Shin, J.-A., Lee, E.-S., Hong, S.-T. (2014)(a). Influence of Ginkgo biloba extracts and of their flavonoid glycosides fraction on the in vitro digestibility of emulsion systems. Food Hydrocolloids. 42:196-203.

Yang, Z., Peng, Z., Li, J., Li, S., Kong, L., Li, P., Wang, Q. (2014)(b). Development and evaluation of novel flavour microcapsules containing vanilla oil using complex coacervation approach. Food Chemistry. 145:272-77.

Yuan, Q., Williams, R.A. (2014). Precision emulsification for droplet and capsule production. Advanced Powder Technology. 25:122-35.

Yucel, U., Elias, R.J., Coupland, J.N. (2013). Localization and reactivity of a hydrophobic solute in lecithin and caseinate stabilized solid lipid nanoparticles and nanoemulsions. Journal of Colloid and Interface Science. 394:20-25.

Zeeb, B., Lopez-Pena, C.L., Weiss, J., McClements, D.J. (2015). Controlling lipid digestion using enzymeinduced crosslinking of biopolymer interfacial layers in multilayer emulsions. Food Hydrocolloids. 46:125-33.

Zeeb, B., Thongkaew, C., Weiss, J. (2014). Theoretical and practical considerations in electrostatic depositioning of charged polymers. Journal of Applied Polymer Science. 131(7):1-11.

Zhang, Z., Zhang, R., Decker, E.A., McClements, D.J. (2015). Development of food-grade filled hydrogels for oral delivery of lipophilic active ingredients: pH-triggered release. Food Hydrocolloids. 44:34552.

Zuidam, N.J., Shimoni, E. (2010). Overview of Microencapsulates for Use in Food Products or Processes and Methods to Make Them. In: Encapsulation Technologies for Active Food Ingredients and Food Processing, Springer Science+Business Media, Dordrecht, pp. 122-35. 


\section{Chapter 3}

Destabilization of multilayered

interfaces in emulsions

This chapter has been published as Corstens, M.N., Berton-Carabin, C.C., Kester, A, Fokkink, R, van der Broek, J.M., de Vries, R., Troost, F.J., Masclee, A.A.M., \& Schroën, K. (2017) Destabilization of multilayered interfaces in digestive conditions limits their ability to prevent lipolysis in emulsions. Food Structure 12:54-63. 


\section{ABSTRACT}

Delivery of lipid fractions in the lower small intestine can induce feelings of satiety, but is only possible when lipids escape the highly efficient lipolysis and adsorption in the upper gastrointestinal (GI) tract. Our objective was to gain insight in the stability of multilayered interfaces in simulated GI conditions, and their suitability for intestinal delivery of undigested lipids. Oil-in-water emulsions ( $d_{32} \sim 5-30 \mu \mathrm{m}$; one- to five-layered interfaces) were produced by sequentially adsorbing biopolymers with opposite charges at $\mathrm{pH}$ 3.0: whey protein isolate (WPI) (cationic), pectin (anionic), chitosan (cationic). Corresponding multilayered structures were characterized using reflectometry. Influence of layer composition and thickness on its protectiveness against lipolysis of emulsions was studied in simulated GI conditions.

Multilayered WPI/pectin emulsions had an improved physical stability compared to WPIstabilized emulsions, during both storage and in vitro gastric incubation, whereas chitosancontaining emulsions were physically unstable. Reflectometry and CLSM results showed that a greater number of layers increased the adsorbed amount, forming a mesoscopically heterogeneous structure. Under simulated intestinal conditions, however, outer layers instantaneously destabilized. Accordingly, similar initial lipolysis rates were recorded for all emulsions. Yet, compared to only WPI the final extent of lipolysis was lowered by addition of a second and a third layer under mild in vitro conditions. This moderate protective effect disappeared when harsher digestive conditions were applied.

From this work, it became clear why multilayered interfaces (initially built under acidic $\mathrm{pH}$ ) can improve gastric stability of emulsions, but are prone to disintegration under intestinal conditions. This knowledge is important for designing food systems that control release of lipolytic products in targeted locations of the GI tract; the emulsions reported here are expected to be suitable for duodenal release. 


\subsection{INTRODUCTION}

A key strategy to control the worldwide expansion of obesity prevalence is by large-scale application of dedicated dietary interventions. These may target, for example, induction of feelings of satiety, thus leading to reduced food intake. Several gastrointestinal (GI) processes are involved in food intake regulation, such as gastric distension, and the sensing of nutrients and their digestion products. Gastric distension triggers mechanosensor-mediated signals and suppresses the release of the stomach hormone ghrelin. The presence of macronutrients in the small intestine induces the release of several gut peptides that are known to be associated with food intake, such as cholecystokinin (CCK), peptide YY (PYY), and glucagon-like peptide-1 (GLP-1) (van Avesaat, Troost, Ripken, Hendriks, \& Masclee, 2015). Especially the presence of nutrients in more distal parts of the small intestine induces the release of gut peptide hormones that control feelings of satiety and hunger in humans, hence reducing food intake (Alleleyn, van Avesaat, Troost, \& Masclee, 2016; Maljaars, Peters, Mela, \& Masclee, 2008). This mechanism is referred to as 'the ileal brake'. For instance, ileal infusion of safflower oil, rich in linoleic acid, has been found to reduce hunger efficiently (Maljaars, Romeyn, Haddeman, Peters, \& Masclee, 2009).

In practice, it is difficult to control lipolysis because the human GI tract has evolved towards efficient food digestion, through a range of processes that allow optimized lipolysis and absorption of digestion products (Bakala N'Goma, Amara, Dridi, Jannin, \& Carrière, 2012). Lipase and bile salts play a major role in this respect. Lipolysis takes place at the surface of lipid droplets, where lipase encounters the lipid substrate (triacylglycerols). At this interface, bile salts play multiple important roles that promote lipolysis: stabilizing new interfacial area, displacing surface-active molecules from the oil-water interface via competitive adsorption, facilitating lipase adsorption at the interface (Maldonado-Valderrama, Wilde, Macierzanka, \& Mackie, 2011). Moreover, unadsorbed bile salts play an even more important role in the aqueous phase, to solubilize digestion products and, hence, prevent enzyme inhibition (Sarkar, Ye, \& Singh, 2016).

The availability of the lipid substrate for lipase is determined not only by the amount of oilwater interfacial area, but also by the interfacial structure. For example, galactolipids (Chu et al., 2009) and Pluronics (Wulff-Pérez, Vicente, Martín-Rodríguez, \& Gálvez-Ruiz, 2012) have been shown to provide a physical barrier against lipolysis in emulsions, via steric hindrance. Yet, this is an exception to the general trend that most emulsifiers that conventionally stabilize food emulsions (low molecular-weight emulsifiers and amphiphilic biopolymers) only have a minor influence on lipolysis (Golding et al., 2011; Hur, Decker, \& McClements, 2009). Recently, specific Pickering particle-stabilized interfaces have been shown to delay lipolysis to a certain extent (Sarkar, Murray, et al., 2016; Tzoumaki, 
Moschakis, Scholten, \& Biliaderis, 2013), but to truly control lipolysis, more protective interfacial structures are required.

A more compact or thicker interfacial structure may be more protective against lipolysis, and can be achieved by for example, combining more than one material. Multilayered interfaces can be produced through layer-by-layer adsorption of oppositely charged biopolymers and is based on electrostatic attraction, with the electrostatic charge alternating after each added layer due to charge overcompensation (Schönhoff, 2003). For example, whey proteins, casein, gum Arabic, modified starch, gelatin, carrageenan, pectin and chitosan have been used previously for that purpose (Dickinson, 2011; Guzey \& McClements, 2006). Whey proteins and pectin have often been combined to create multilayered interfaces (Mao \& Miao, 2013; Wackerbarth, Schön, \& Bindrich, 2009; Zhang \& Zhong, 2015), and layers close to the oil-water interface have been suggested to become strongly intermingled due to the attraction between oppositely charged molecules, causing a dense packing (Wackerbarth et al., 2009). In addition, a greater number of layers would increase the distance over which functional ingredients, such as fatty acids and vitamins, need to be transported across the interface, which may also lead to additional protection against digestion (Wackerbarth et al., 2009). A nice example of mechanically strong multilayered capsules can be found in the work of Rossier-Miranda and co-workers (Rossier-Miranda, Schroën, \& Boom, 2010), who combined pectin with whey protein fibrils to fortify the interfacial shell. These capsules were physically stable at low $\mathrm{pH}(\mathrm{pH} 2.0-3.5)$, while they disintegrated more rapidly at high $\mathrm{pH}$ (pH 7.0), because of the loss of charge interactions.

Multilayered emulsions have already been studied in relation to lipolysis, but most studies found only a minor influence of additional layers on in vitro lipolysis (Corstens et al., 2017). It is difficult to compare the results published so far, notably because of the broad range of experimental conditions used. Some studies found that a chitosan outer layer delayed lipolysis of emulsions (Klinkesorn \& McClements, 2010; Mun, Decker, Park, Weiss, \& McClements, 2006), and this was attributed to the formation of a thick cationic layer restricting lipase adsorption, although this was not systematically confirmed (Li \& McClements, 2014). In some studies, adsorbing a second layer facilitated lipolysis (Lesmes, Baudot, \& McClements, 2010; Tokle, Mao, \& McClements, 2013), which was attributed to enhanced removal of additional layers from the surface while passing through different stages of the GI tract, compared with single layer material. Conversely, other studies found that a second layer formed a more protective interfacial structure compared with primary emulsions, resulting in delayed lipolysis (Hu, Li, Decker, Xiao, \& McClements, 2011; Klinkesorn \& McClements, 2010; Li et al., 2010; Mun et al., 2006). Hence, it was not possible to draw consistent conclusions from available studies on a potential protective effect of multilayered emulsions with respect to lipolysis. 
To elucidate the underlying mechanisms, we systematically investigated the structure of multilayered interfaces in emulsions and on model surfaces, and the in vitro gastric resistance and intestinal stability against lipolysis of emulsified dietary lipids. Oil-in-water (O/W) emulsions were produced with a different layer composition and thickness, using whey proteins in combination with pectin, and in combination with chitosan. We performed a detailed characterisation of the interfacial structure using, amongst others, confocal fluorescence microscopy and reflectometry under digestion-relevant conditions. In vitro lipolysis studies were performed under various conditions and we were able to relate these results to the interfacial composition and thickness.

\subsection{MATERIAL AND METHODS}

\subsubsection{Materials}

Safflower oil was purchased from De Wit Specialty oils (19200 Safflower Oil High Linoleic Refined, The Netherlands). Three biopolymers were used: whey protein isolate (WPI) (BiPro, Davisco Food International, Eden Prairie, Minnesota, USA; purity 97.5\%), pectin from citrus peel ( $\geq 74 \%$ galacturonic acid, $\geq 6.7 \%$ methoxy groups, Sigma Aldrich, St. Louis, MO, USA) and low molecular weight chitosan (75-85\% deacetylated, Sigma Aldrich, St. Louis, MO, USA). From Sigma Aldrich (St. Louis, MO, USA) we also purchased: citric acid, calcium chloride, sodium citrate dihydrate, sodium hydroxide, sodium chloride, sodium phosphate dibasic, sodium phosphate monobasic, fluorescein isothiocynate isomer I (FITC), 4-morpholineethanesulfonic acid monohydrate, $N$-(3-dimethylaminopropyl)- $N$ 'ethylcarbodiimide hydrochloride (EDC), $N$-hydroxysulfosuccinimide sodium salt (SulfoNHS), hexamethyldisilazane (HMDS), toluene, hexane, 2-propanol, pepsin from porcine gastric mucosa, lipase from porcine pancreas, pancreatin from porcine pancreas $(8 \times$ USP specification; including trypsin, amylase, lipase, ribonuclease, protease), porcine bile extract (crude extract, purity estimated to be 30-60\%, containing glycine and taurine conjugates of hyodeoxycholic acid and other bile salts according to the supplier) and phenolphthalein reagent. Enzyme activities were measured according to Minekus et al. (2014), and found to be $48 \mathrm{U} \cdot \mathrm{mg}^{-1}$ for lipase from porcine pancreas, $41 \mathrm{U} \cdot \mathrm{mg}^{-1}$ for pancreatin from porcine pancreas, and $>400 \mathrm{U} \cdot \mathrm{mg}^{-1}$ for the used pepsin. Ethanol (absolute, for analysis) was purchased from Merck (Amsterdam, The Netherlands). All materials were used directly without further purification. Ultrapure water obtained from a Millipore Milli-Q system (Darmstadt, Germany) was used throughout the study. 


\subsubsection{Methods}

\section{Preparation of emulsions}

Primary WPI-stabilized emulsion. WPI solution $\left(10 \mathrm{~g} \mathrm{~L}^{-1}\right)$ was prepared in $10 \mathrm{mM}$ citric acid buffer $\mathrm{pH}$ 3.0, gently stirred for at least $8 \mathrm{~h}$ at room temperature and undissolved material (<3 wt\%) was removed by centrifugation (30 min at $4800 \times \mathrm{g}, 20^{\circ} \mathrm{C}$, ThermoScientific, Legend XFR). Safflower oil was mixed with diluted WPI solution (with a final lipid concentration of $10 \mathrm{wt} \%$ and $0.5 \mathrm{wt} \% \mathrm{WPI}$ in the mixture) using a rotor-stator homogeniser (Ika T18 basic Ultra-Turrax homogenizer equipped with a S18N-19G dispersion tool, Staufen, Germany) two times for $30 \mathrm{~s}$ at 7,000 rpm, with a $30 \mathrm{~s}$ break, to obtain a coarse emulsion. The coarse emulsion was then passed three times through a premix emulsification column (5 bar, nickel sieve with a thickness of $400 \mu \mathrm{m}$, rectangular pores $(11.6 \times 331 \mu \mathrm{m})$, and a porosity of 0.04 , Stork Veco, The Netherlands) to obtain a fine primary WPI-stabilized emulsion; for more details on premix emulsification, the work of Nazir and co-workers is suggested (Nazir, Schroën, \& Boom, 2010).

Layer-by-layer assembly. The primary WPI-stabilized emulsion was added dropwise to a pectin solution (10 $\mathrm{g} \mathrm{L}^{-1}$ in $10 \mathrm{mM}$ citric acid buffer $\left.\mathrm{pH} 3.0\right)$ and stirred for at least $30 \mathrm{~min}$ to allow for adsorption. After that, the mixture was mildly centrifuged ( $1 \mathrm{~h}$ at $70 \times \mathrm{g}, 20^{\circ} \mathrm{C}$, ThermoScientific, Legend XFR) to collect the oil droplets in a creamed layer, which was gently washed by re-dispersing in $10 \mathrm{mM}$ citric acid buffer $\mathrm{pH} 3.0$ to remove excess biopolymer, and once repeating the centrifugation-collecting step. After two washing steps, the two-layered emulsion (redispersed in $10 \mathrm{mM}$ citric acid buffer $\mathrm{pH} 3.0$; at $10 \mathrm{wt} \%$ lipid) was added dropwise to a WPI solution $\left(10 \mathrm{~g} \mathrm{~L}^{-1}\right.$ in $10 \mathrm{mM}$ citric acid buffer $\left.\mathrm{pH} 3.0\right)$ or chitosan solution (10 $\mathrm{g} \mathrm{L}^{-1}$ in $10 \mathrm{mM}$ citric acid buffer $\mathrm{pH}$ 3.0). The next layers were adsorbed following the same procedure; emulsions were stirred for at least $30 \mathrm{~min}$ to allow adsorption to take place; designed emulsions are summarized in Table 3.1. During all adsorption steps, the lipid concentration was about $5 \mathrm{wt} \%$ and the concentration of layer material was $0.5 \mathrm{wt} \%$.

\section{Physical characterization of emulsions}

Droplet size distribution. The droplet size distribution of the emulsions was determined using a static light scattering instrument (Mastersizer 2000 with Hydro SM dispersion unit, Malvern Instruments, UK). The refractive index of safflower oil was set at 1.460 and the refractive index of the dispersant at 1.333 .

Emulsion morphology. The morphology of the emulsions was characterized using light microscopy (Zeiss Axioscope A1, Axiocam Mrc 5), at 100-time magnification. The samples were not diluted, and the microscope slides were freshly prepared. To obtain a general overview of the sample, multiple images were analysed in various locations of the slides. 
Structural organisation of protein layers in emulsions. Whey proteins were stained with fluorescein isothiocynate isomer I (FITC; $75 \mathrm{mg} \cdot \mathrm{g}^{-1}$ protein) via $\mathrm{N}$-(3dimethylaminopropyl)- $N$ '-ethylcarbodiimide hydrochloride $\quad$ (EDC)/ Nhydroxysulfosuccinimide sodium salt (Sulfo-NHS) coupling (using the recommended protocol of Thermo Scientific). Free molecules were removed by dialysis (cellulose membrane, cut-off $14 \mathrm{kDa}$, Sigma Aldrich, St. Louis, MO, USA) before the stained WPI solution was used to prepare one- to five-layered emulsions. The samples were observed within $36 \mathrm{~h}$ after production using a confocal laser-scanning microscope (CLSM; Carl Zeiss Axiovert microscope, equipped with a LSM 5 Exciter configuration and a 100-times objective, Zeiss, Germany). FITC was excited by an argon-laser (488 nm; at 20\% of the maximal laser power $25 \mathrm{~mW}$ ) and the signal was modified with a dichroic mirror 488, beam splitter 490 and low pass filter 505 before collection in the detector (gain 700; pinhole of 1 airy unit).

Droplet surface charge. The droplet surface charge ( $\zeta$-potential, $\mathrm{mV}$ ) was determined using dynamic light scattering (Zetasizer Nano ZS, Malvern Instruments, UK). The emulsions were diluted 300 times in $10 \mathrm{mM}$ citric acid buffer pH 3.0 to avoid multiple scattering effects.

Oil content. The oil content needed to be determined since washing steps in the preparation of multilayered emulsions may result in minor oil loss. A known amount of emulsion ( $\pm 1 \mathrm{~g}$ ) was added to a known amount of hexane/2-propanol (3:2 (v/v) ratio) mixture ( $\pm 15 \mathrm{~g})$, mixed (hand shaken, and vortexed twice for $15 \mathrm{~s}, 3,000 \mathrm{rpm}$ ) and then centrifuged (5 min at $2,000 \times \mathrm{g}, 20{ }^{\circ} \mathrm{C}$, ThermoScientific, Legend XFR) to separate into two phases. A known amount of the hexane phase containing the extracted oil was dried and the amount of oil in the initial emulsion was calculated from a mass balance.

\section{Behaviour under simulated digestive conditions}

pH stability of emulsions. Emulsions were diluted 4 times in $10 \mathrm{mM}$ phosphate buffer $\mathrm{pH}$ 7.0 , in a double walled beaker, kept at $37^{\circ} \mathrm{C}$, and sequentially brought to $\mathrm{pH}$ values that they would face in the GI compartments: the mouth ( $\mathrm{pH} 7.0$ ), the stomach ( $\mathrm{pH} 2.0$ ), the duodenum ( $\mathrm{pH}$ 5.3) and the distal small intestine ( $\mathrm{pH} 7.0$ ). We adjusted the $\mathrm{pH}$ with $0.1 \mathrm{M} \mathrm{HCl}$ or $0.1 \mathrm{M}$ $\mathrm{NaOH}$ and stirred for $20 \mathrm{~min}$ at $240 \mathrm{rpm}$ between each step. The microscopic appearance was recorded at the beginning and at the end of each step, and the droplet size distribution was measured using static light scattering.

Gastric stability of emulsions. Emulsions were exposed to simulated gastric fluid consisting of $0.5 \mathrm{mg} \cdot \mathrm{mL}^{-1}$ pepsin, $150 \mathrm{mM} \mathrm{NaCl}$ and $10 \mathrm{mM} \mathrm{CaCl}_{2}$ (pH of 3.0) for $2 \mathrm{~h}$ at $37{ }^{\circ} \mathrm{C}$. The total amount of safflower oil was $0.3 \mathrm{~g}$ in a total volume of $20 \mathrm{~mL}$. The droplet size and appearance of undiluted samples from this gastric fluid was followed in time using light microscopy, and multiple images were made. 
Adsorption and desorption of biopolymer layers. In order to quantify adsorption and desorption of biopolymer layers, reflectometry experiments were performed in conditions simulating the formation of multilayers at $\mathrm{pH}$ 3.0, followed by exposure to $\mathrm{pH} 7.0$ (intestinal conditions). The principle and procedure for fixed angle stagnation point reflectometry is described in detail in the work of Dijt, Stuart, \& Fleer, (1994). In short, a linearly polarised He-Ne beam $(\lambda=632.8 \mathrm{~nm})$ is reflected by an adsorbing surface and the state of polarisation is measured by simultaneous detection of the parallel $\left(I_{p}\right)$ and perpendicular intensity $\left(I_{s}\right)$. The ratio of the intensities $\left(S=I_{p} / I_{S}\right.$ ) changes upon adsorption of material onto the surface, and the relative change in output signal $d S / S_{o}$ can be related to the adsorbed mass per unit area $\Gamma$ using a conversion factor $Q$. This factor is defined to match $\Gamma=d S / S_{o} \cdot Q$, and is based on the refractive index increments $(d n / d c)$ of the material and the thickness of the adsorbed layer. Since the value of $Q$ was unknown for our multilayered biopolymer system, we expressed our results as $d S / S_{o}$ data in the result section. Yet, we calculated the $Q$ factor in an optical model based on Abeles Matrix Formalism in Professor Huygens software $(Q=22 \pm 4$ $\left.\mathrm{mg} \cdot \mathrm{m}^{-2}\right)$ to give a rough estimate of the adsorbed amount, defined as $\Gamma^{*}\left(\mathrm{mg} \cdot \mathrm{m}^{-2}\right)$.

Model surfaces of $1 \mathrm{~cm} \times 4 \mathrm{~cm}$ were made from clean wafers (polished silicon wafer, diameter 150 mm; Siltronic, München, Germany) onto which a silica layer was created first (heating for $100 \mathrm{~min}$ at $1,000{ }^{\circ} \mathrm{C}$ ) and then made hydrophobic with HMDS to obtain comparable properties as the safflower oil droplet surface (Maccarini, Himmelhaus, Stoycheva, \& Grunze, 2005). Optical ellipsometry (Sentech SE400) was used to determine the properties of the oxide layer (62 nm silica, reflective index 1.455).

The reflectometry signal $S$ was monitored in time while flushing biopolymer solutions (500 ppm WPI and pectin, $\mathrm{pH} 3.0$ ) at $2 \mathrm{~mL} \cdot \mathrm{min}^{-1}$ over the model surfaces onto which subsequent layers were deposited. The adsorption steps were always $15 \mathrm{~min}$ for the first, second and fourth layer and 30 min for the third and fifth layer to allow the adsorbed amount to reach its maximum value. After each adsorption step the surface was rinsed with solvent (10 mM citric acid buffer $\mathrm{pH}$ 3.0) for 15 min to remove loosely attached components. After the required number of layers was obtained, the $\mathrm{pH}$ was changed $(10 \mathrm{mM}$ phosphate buffer $\mathrm{pH}$ 7.0), and the stability of the layers was tested.

Intestinal lipolysis. Lipolysis was measured based on the release of free fatty acids (FFA) in a static in vitro digestion model that represented the intestinal conditions in fasted state ( $\mathrm{Li}$, $\mathrm{Hu}, \&$ McClements, 2011), unless otherwise stated. The digestion was performed at $37^{\circ} \mathrm{C}$ in a double-walled reaction vessel connected to a water bath, and stirred at a rate of $240 \mathrm{rpm}$. The reaction fluid was prepared by adding $25.5 \mathrm{~mL} 10 \mathrm{mM}$ phosphate buffer $\mathrm{pH}$ 7.0, $2 \mathrm{~mL}$ salt solution (2.8 M NaCl, $0.2 \mathrm{M} \mathrm{CaCl}_{2}$ in $10 \mathrm{mM}$ phosphate buffer $\mathrm{pH} 7.0$ ), $5 \mathrm{~mL}$ fresh bile extract solution $\left(187.5 \mathrm{mg} \cdot \mathrm{mL}^{-1}\right)$ and $2 \mathrm{~mL}$ fresh lipase solution $\left(7.5 \mathrm{mg} \cdot \mathrm{mL}^{-1}\right)$ to the vessel. 
When the reaction fluid reached $37^{\circ} \mathrm{C}$, digestion was started by adding $3 \mathrm{~mL}$ emulsion ( $~ 10 \mathrm{wt} \%$ oil) to the reaction vessel. The final composition of the digestive fluid was 5 $\mathrm{mg} \cdot \mathrm{mL}^{-1}$ bile salts, $0.4 \mathrm{mg} \cdot \mathrm{mL}^{-1}$ lipase, $10 \mathrm{mM} \mathrm{CaCl}_{2}, 150 \mathrm{mM} \mathrm{NaCl}, 0.3 \mathrm{mg}$ lipid and $9 \mathrm{mM}$ phosphate buffer $\mathrm{pH}$ 7.0. After digestion started, the $\mathrm{pH}$ was not automatically controlled and allowed to decrease a bit (typically not below pH 6.7).

In vitro lipolysis was determined as well in a simulated intestinal fluid representing the fed state, for which the protocol was the same as described above, except that the final digestion fluid contained $2.4 \mathrm{mg} \cdot \mathrm{mL}^{-1}$ lipase and $20 \mathrm{mg} \cdot \mathrm{mL}^{-1}$ bile (Li, Hu, \& McClements, 2011). In addition, in vitro digestions were performed with pancreatin (containing a broader range of enzymes than the purified lipase used) instead of pure lipase, under fed state conditions (2.4 $\mathrm{mg} \cdot \mathrm{mL}^{-1}$ pancreatin; $20 \mathrm{mg} \cdot \mathrm{mL}^{-1}$ bile).

Determination of the FFA content. To quantify lipolysis, the FFA content was determined in time by a titration method. At different time points ( $\mathrm{t}=0,5$ 15, 30, 50, 90 and $150 \mathrm{~min}$ ) samples of $3.5 \mathrm{~g}$ were taken from the digestion vessel. The sample was immediately diluted in ethanol (12 g) to inactivate the enzymes and to solubilize the FFAs. The ethanol contained phenolphthalein (0.04 wt\%) as indicator, and the samples were then titrated with $15 \mathrm{mM}$ $\mathrm{NaOH}$ solution. Lipolysis was expressed as the percentage of released free fatty acids, which was calculated based on the maximum release of two FFAs from one triacylglycerol molecule (Equation 3.1):

$$
\% F F A=100 \cdot\left(\frac{V_{\mathrm{NaOH}} \cdot M_{\mathrm{NaOH}}}{w_{\text {sample }}}\right) \cdot\left(\frac{w_{D F} \cdot M W_{\text {lipid }}}{w_{\text {lipid }} \cdot 2}\right)
$$

Equation 3.1

where $V_{\mathrm{NaOH}}$ and $M_{\mathrm{NaOH}}$ are the volume (L) and molarity (mol L $\mathrm{L}^{-1}$ ) of sodium hydroxide used to neutralize the sample, $M W_{\text {lipid }}$ the average molecular weight of the lipid material present (estimated to be $871 \mathrm{~g} \cdot \mathrm{mol}^{-1}$ ), $w_{\text {lipid }}$ the total weight of lipid initially present in the reaction vessel ( $\sim 0.3 \mathrm{~g}$; based on the oil measurement as explained before), $w_{D F}$ the total mass of digestive fluid ( $\mathrm{g}$ ) and $w_{\text {sample }}$ the mass of sample taken from the digestive fluid for analysis (g).

\section{Experimental design}

For each emulsion shown in Table 3.1, we characterized the oil content, light microscopy, $\zeta$ potential, and particle size distribution in time (at $20^{\circ} \mathrm{C}$ ) in two independently prepared emulsions. Within each measurement of $\zeta$-potential and particle size, we recorded three values. The CLSM and reflectometry experiments were performed on two independent replicates. The behaviour in vitro under simulated digestive conditions representing the gastric phase, and the intestinal fasted state and the fed state (with pancreatin or more pure 
lipase) was studied in two independently prepared emulsions. The droplet size $\left(d_{32}\right)$ prior to intestinal digestion was used to calculate the available surface area during incubation in simulated intestinal fluid. This surface area was used to estimate initial free fatty acid release rate (per square meter). Average values with standard deviations are shown in the results and in the figures for the $\zeta$-potential, droplet size, FFA release curves and reflectometry experiments.

\subsection{RESULTS AND DISCUSSION}

\subsubsection{Physical characterisation of emulsions}

Surface charge, droplet size and microscopic appearance

Emulsions stabilised with one to five interfacial layers were prepared at $\mathrm{pH} 3.0$ using positively charged whey proteins (WPI), negatively charged pectin, and either positively charged chitosan or WPI. Primary WPI-stabilized emulsions had a $d_{32}$ of about $5 \mu \mathrm{m}$, contained many small droplets (1-4 $\mu \mathrm{m}$; Supplementary Figure 3.8), and had a high $\zeta$ potential $(+39.7 \pm 4.3 \mathrm{mV})$. After adsorption of each additional layer, the net surface charge reversed, which confirmed adsorption of the next layer component (Table 3.1). The adsorbed amounts are discussed later, in section 3.2.2 on reflectometry.

Table 3.1. Overview of compositions of prepared emulsions. The sign between brackets indicates the global electrostatic charge of the layer material at $\mathrm{pH}$ 3.0. The $\zeta$-potential represents the average overall surface charge of the emulsions at $\mathrm{pH} 3.0$, with standard deviation $(n=2)$.

\begin{tabular}{lcccccc}
\hline Sample Layer: & $\mathbf{1}$ & $\mathbf{2}$ & $\mathbf{3}$ & $\mathbf{4}$ & $\mathbf{5}$ & $\zeta$-potential (mV) \\
\hline Primary emulsion & WPI (+) & & & & & $+39.7 \pm 4.3$ \\
W-P emulsion & WPI (+) & P (-) & & & & $-9.4 \pm 0.7$ \\
W-P-W emulsion & WPI (+) & P (-) & WPI (+) & & & $+6.0 \pm 1.3$ \\
W-P-C emulsion & WPI (+) & P (-) & C (+) & & & $+39.0 \pm 0.5$ \\
W-P-W-P emulsion & WPI (+) & P (-) & WPI (+) & P (-) & & $-8.6 \pm 0.7$ \\
W-P-C-P emulsion & WPI (+) & P (-) & C (+) & P (-) & & $-8.7 \pm 0.4$ \\
W-P-W-P-W emulsion & WPI (+) & P (-) & WPI (+) & P (-) & WPI (+) & $+9.7 \pm 1.3$ \\
\hline
\end{tabular}

Abbreviations: $W=$ whey protein isolate (WPI), $P=$ pectin, $C=$ chitosan.

The outer layer determined the sign of the charge: positive for WPI or chitosan, and negative for pectin, in accordance with previous work (Rossier-Miranda et al., 2010; Zhang \& Zhong, 2015). The magnitude of the net charge depended on the overall layer composition; emulsions with a WPI outer layer had a lower net $\zeta$-potential (about +6 to $+10 \mathrm{mV}$ ) compared with primary WPI-stabilized emulsions $(+39.7 \pm 4.3 \mathrm{mV})$. This lower charge can result from a 
lower tendency of native proteins to reorganize when adsorbing at a pre-coated biopolymer surface, compared with the bare oil-water interface (Dickinson, 2011; Schönhoff, 2003).

In most multilayered emulsions, individual droplets were observed (Supplementary Figure 3.8). The mean size of multilayered emulsions ( $d_{32}$ about $10-17 \mu \mathrm{m}$ ) was about two-to-three times greater than that of primary emulsions, due to the absence of the small droplet population (around 1-4 $\mu \mathrm{m}$ ). These small droplets were present in the subnatant following the centrifugation step (to collect the cream phase), and were therefore discarded. It should be pointed out that the thickness of multilayered structures did not affect the mean droplet size, as a layer thickness of $<0.1 \mu \mathrm{m}$ was reported for five-layered WPI/pectin structures (Rossier-Miranda et al., 2010).

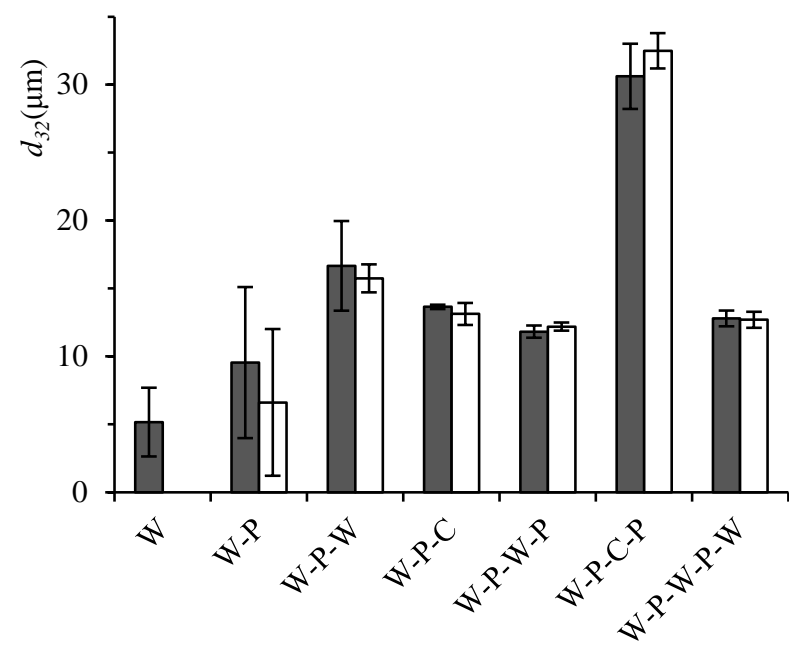

Figure 3.1. Sauter diameter $\left(d_{32}\right)$ of multilayered emulsions, fresh (filled bars) and 7 days old (empty bars) $(n=2)$. $\mathrm{W}=$ whey proteins, $\mathrm{P}=$ pectin, $\mathrm{C}=$ chitosan.

The W-P-C-P emulsions had a $d_{32}$ of about $30 \mu \mathrm{m}$ and were clearly flocculated, most probably due to bridging flocculation (McClements, 2005), between pectin molecules and highly charged chitosan molecules on the outside of W-P-C droplets $(+39.7 \pm 4.3 \mathrm{mV}$, Table 3.1), which makes them less suitable candidates to form a multilayered interface structure compared to emulsions with only WPI/pectin.

To determine the physical stability of emulsions, we measured the $d_{32}$ (Figure 3.1) and visualized their microscopic appearance (Supplementary Figure 3.8) after $7 \mathrm{~d}$ storage $\left(20^{\circ} \mathrm{C}\right)$. Primary emulsions were not physically stable and showed a thin layer of oil floating on top (that cannot be captured in a value so is left out in Figure 3.1). Multilayered emulsions, in contrast, remained physically stable over $7 \mathrm{~d}$ storage. This cannot result from electrostatic 
repulsion between droplets, as multilayered emulsions had a lower surface charge (about -9 to $+10 \mathrm{mV}$, Table 3.1) than primary emulsions (+39.7 $\pm 4.3 \mathrm{mV})$. Most probably, the good physical stability of multilayered emulsions was due to steric stabilization and the ability of the biopolymer combinations to form thick, viscoelastic films.

$\underline{\text { Structural organisation of interfacial whey proteins in emulsions }}$

Protein location within multilayered emulsions was visualized by CLSM (excitation $488 \mathrm{~nm}$ ) using WPI stained with FITC. Figure 3.2 shows images of one- to five-layered emulsions. A bright yellow ring surrounded all emulsion droplets, indicating the presence of proteins. A structurally homogeneous protein layer was visible at the surface of primary emulsion droplets (Figure 3.2 W) and two-layered emulsion droplets (Figure 3.2 W-P). The background of emulsions with two or more layers appeared dark because of the washing steps that removed most unadsorbed proteins, which were still present in primary WPI-stabilized emulsions.
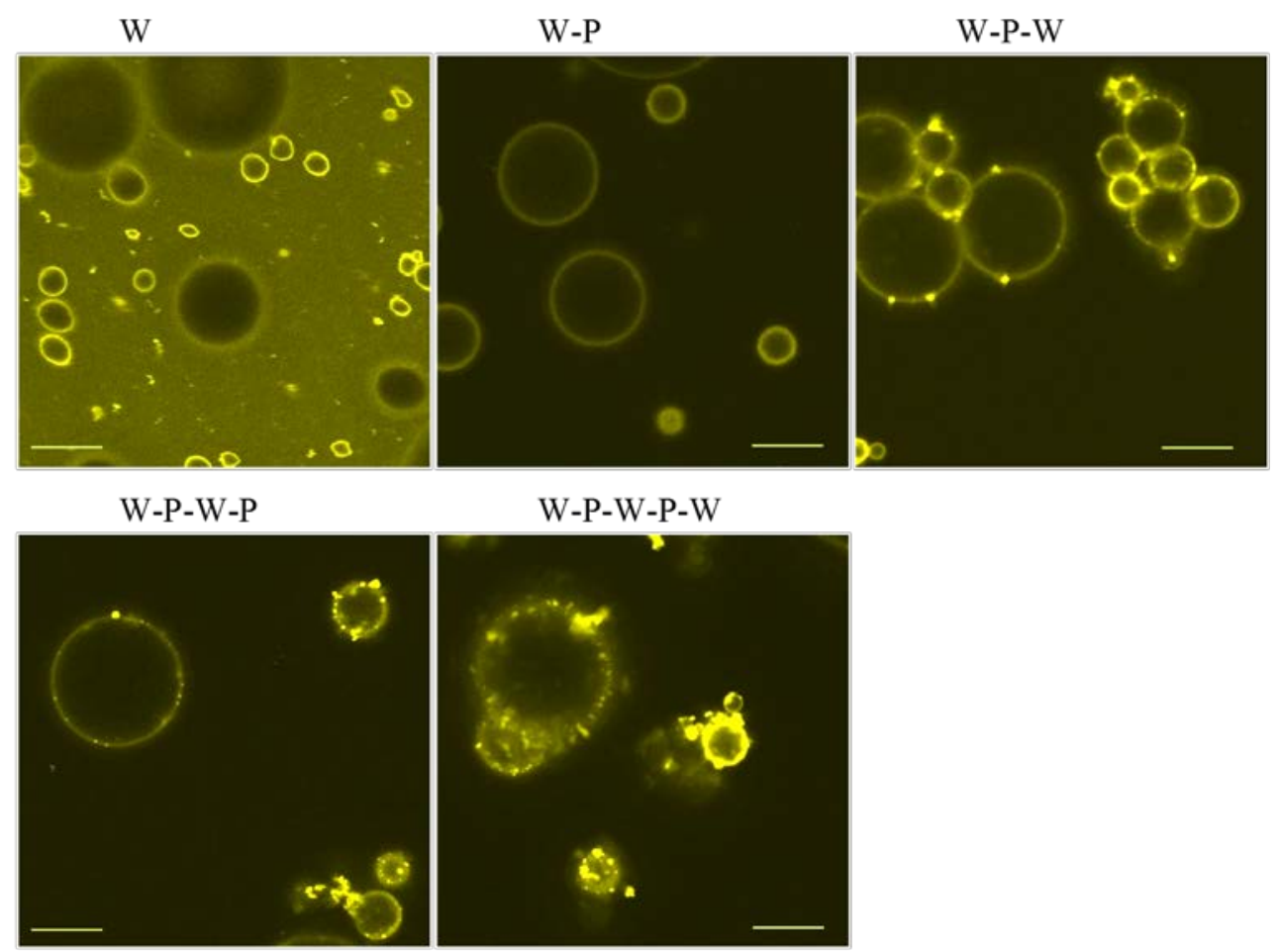

Figure 3.2. CLSM images of multilayered emulsions; excitation was at $488 \mathrm{~nm}$; scale bar represents $10 \mu \mathrm{m}$ (total picture $65 \mu \mathrm{m} \times 65 \mu \mathrm{m}$ ). $\mathrm{W}=$ whey proteins stained with FITC, $\mathrm{P}=$ pectin. 
At the surface of emulsion droplets with three layers (Figure $3.2 \mathrm{~W}-\mathrm{P}-\mathrm{W}$ ), four layers

(Figure 3.2 W-P-W-P), and especially five layers (Figure $3.2 \mathrm{~W}-\mathrm{P}-\mathrm{W}-\mathrm{P}-\mathrm{W}$ ), a heterogeneous layer was visible, with local large protein structures that protruded into the continuous phase, which were also observed by others for comparable multilayered emulsions (Wackerbarth et al., 2009). These large protein structures may be formed due to restructuring within the multilayers, resulting in compaction of proteins (Wackerbarth et al., 2009). Alternatively, large protein structures may also be formed due to phase separation between protein-rich phases and pectin-rich phases as a result of mixing incompatibility, as may occur in a dense structure due to the ability of molecules to move (Dickinson, 2011).

\subsubsection{Behaviour under simulated digestive conditions}

$\mathrm{pH}$ stability of multilayered emulsions

As mentioned, multilayered emulsions were produced at $\mathrm{pH} 3.0$ to provide gastric stability, and biopolymers were selected to have opposite charge at that $\mathrm{pH}$. When passing through the GI tract, however, an emulsion droplet will face various $\mathrm{pH}$ conditions: an oral $\mathrm{pH}$ of 7.0, an extreme gastric $\mathrm{pH}$ of 2.0, a duodenal $\mathrm{pH}$ of 5.3 and an ileal $\mathrm{pH}$ of 7.0. The behaviour of emulsions in these sequential $\mathrm{pH}$ conditions is illustrated in Figure $\mathbf{3 . 3}$ for two emulsions with the same interfacial composition, except for the third layer (WPI or chitosan).

Figure 3.3. Light microscopy images of three-layered emulsions before and after incubation in the indicated $\mathrm{pH}$ conditions. The black bar represents $20 \mu \mathrm{m}$. The average droplet size was determined using static light scattering. $\mathrm{W}=$ whey proteins, $\mathrm{P}=$ pectin, $\mathrm{C}=$ chitosan.

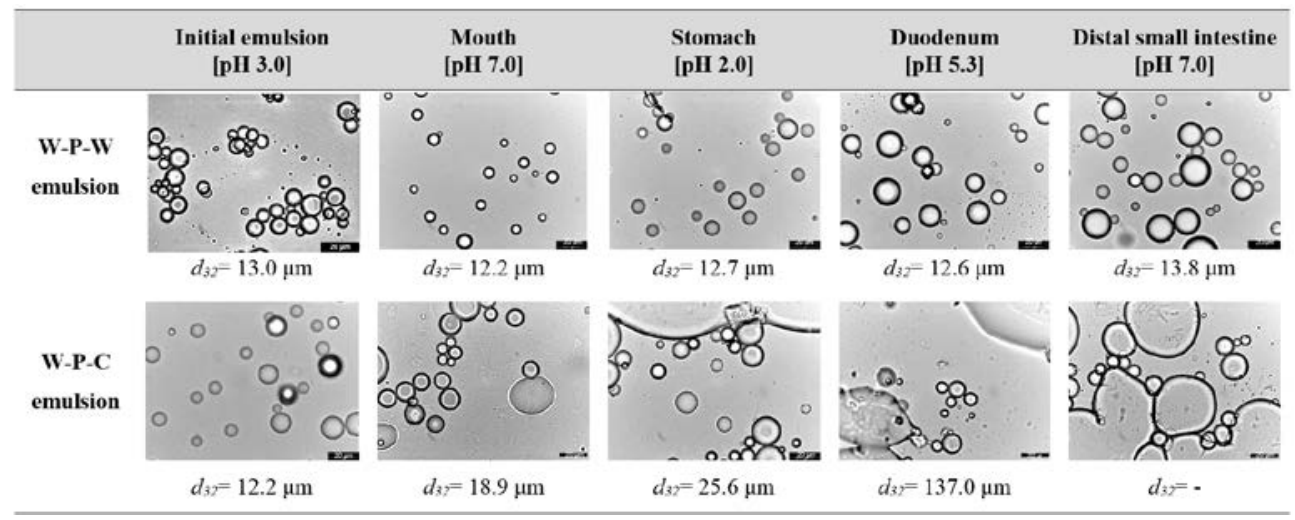

The W-P-C droplets showed extensive coalescence over $\mathrm{pH}$ variation, leading to large oil structures of irregular shape, so these emulsions were greatly unstable. At higher $\mathrm{pH}$, chitosan has less ionized groups ( $p K_{a} \sim 6.5$ (Aoki, Decker, \& McClements, 2005)), which probably caused some disintegration of the layered structure. This can in turn, lead to either droplet flocculation if sufficient charge neutralization took place (Guzey \& McClements, 2006), or 
coalescence if the interconnected interfacial structure was disrupted. Beta-lactoglobulinstabilized emulsions with a chitosan-outer layer have also been reported to flocculate at neutral pH (Li et al., 2010), which was speculated to be caused by chitosan desorption as a result of charge loss. This could, however, be prevented through a third layer of pectin or alginate (Aoki et al., 2005; Li et al., 2010).

The droplet size of multilayered emulsions with only WPI and pectin remained overall unaffected by $\mathrm{pH}$ variations, indicating physical stability. The mouth and distal small intestine have a neutral $\mathrm{pH}$ of about 7.0, which is above the isoelectric point of WPI that is typically $~ 5$ (Golding et al., 2011), and the $p K_{a}$ of pectin that is typically 3.0-4.5. At pH 7.0, both WPI and pectin molecules have a negative charge, and repulsion may occur, resulting in desorption of biopolymers and disintegration of the layered structure. The images in Figure 3.3, however, show individual droplets so did not point in this direction. The stomach has an acidic pH, below the isoelectric point of WPI, and Figure 3.3 illustrates that protein/pectin-based emulsions were stable against coalescence under these conditions. Stability to the acidic gastric conditions has been related to an adaptation in physiological response, which can induce more satiety compared to acid-unstable emulsions (Marciani et al., 2007, 2009).

Because of this better physical $\mathrm{pH}$ stability of whey protein/pectin-based emulsions compared to chitosan-containing emulsions, only these systems were considered for further investigation of their interfacial behaviour in simulated digestive conditions.

\section{Adsorption-desorption kinetics}

The $\mathrm{pH}$ stability of multilayered interfaces was investigated by reflectometry; whey protein and pectin layers were sequentially adsorbed on a hydrophobic surface, after which they were exposed to $\mathrm{pH}$ 7.0, which corresponds to the intestinal $\mathrm{pH}$. Figure 3.4a shows the output signal in time during build-up of the multilayers, which gives semi-quantitative information about the adsorbed amount $\left(\Gamma^{*}\right)$, as shown on the secondary Y-axis.

Adsorption of the first whey protein layer resulted in a surface load of about $1 \mathrm{mg} \cdot \mathrm{m}^{-2}$, as expected for dairy proteins, and this value increased upon adsorption of the next layers, until about $11 \mathrm{mg} \cdot \mathrm{m}^{-2}$ after adsorption of the fifth layer (whey proteins). The increase in surface load seemed to be less when adsorbing pectin compared to whey proteins. Besides, more distant protein layers added more to the total (surface load increased for the first layer by about $1 \mathrm{mg} \cdot \mathrm{m}^{-2}$, for the third by $2 \mathrm{mg} \cdot \mathrm{m}^{-2}$ and for the fifth by $6 \mathrm{mg} \cdot \mathrm{m}^{-2}$ ). This nonlinear increase may be explained by the relatively low electrical charge on the biopolymers, which in turn requires a greater adsorbed mass to reverse the charge (Acevedo-Fani, Salvia-Trujillo, Soliva-Fortuny, \& Martín-Belloso, 2015). The biopolymers in the first layers are more likely 
to adopt a favourable conformation leading to better charge complexation, while in outer layers there is less driving force for that (Schönhoff, 2003), which is in line with the observed structure by CLSM, and can also be seen in the adsorption timescales. Equilibrium was reached faster for the first protein layer compared with the third, and was even slower for the fifth layer, indicating a lower driving force.

a.

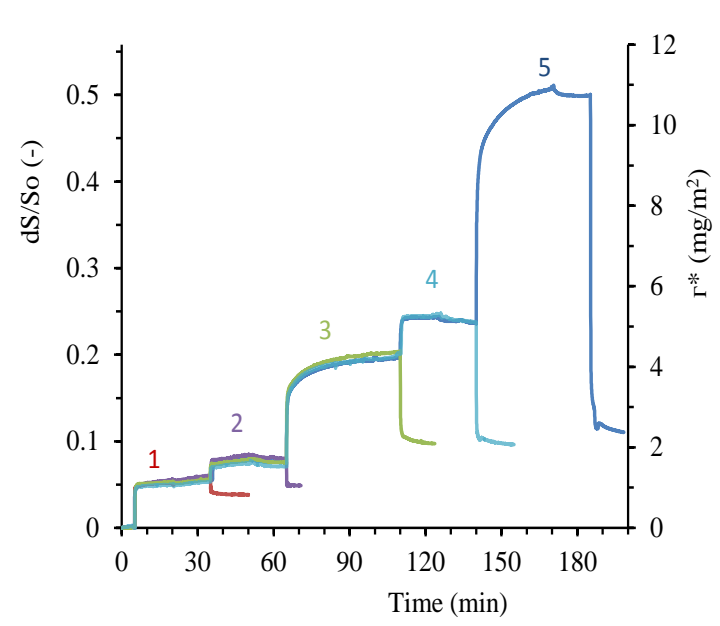

b.

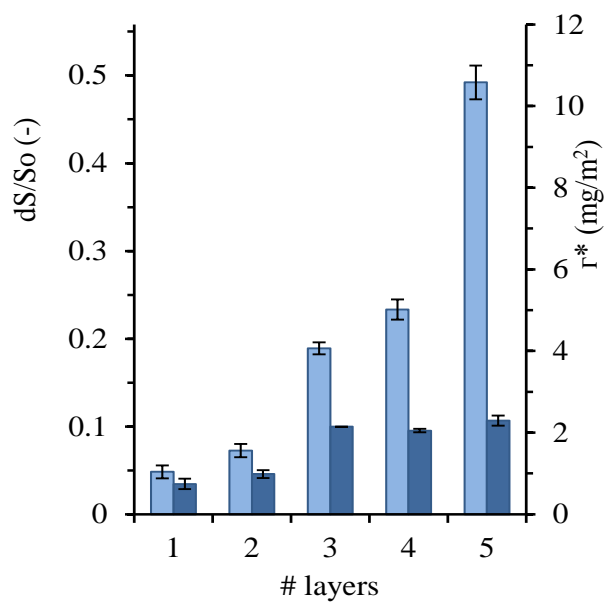

Figure 3.4. a) In situ layer-by-layer adsorption at $\mathrm{pH} 3.0$ and consecutive effect of $\mathrm{pH} 7.0$ as investigated by reflectometry of one- to five-layered films of whey proteins (layer 1, 3 and 5) and pectin (layer 2 and 4), number of layers indicated and shown with different colours. b) Equilibrium values at pH 3.0 (left bars, light colour) and pH 7.0 (right bars, darker colour) $(n=2)$. Each adsorption step was followed by a rinsing step with $10 \mathrm{mM}$ citric acid buffer $\mathrm{pH}$ 3.0. The secondary axis gives an estimate of the adsorbed amount $\left(\Gamma^{*}, \mathrm{mg} \cdot \mathrm{m}^{-2}\right)$.

It is clear that the layers were very stable at $\mathrm{pH}$ 3.0; however, when exposed to $\mathrm{pH} 7.0$ (to emulate the conditions during the intestinal phase) the adsorbed amount reduced dramatically (see Figure 3.4a and $\mathbf{b}$ for average maximum $\Gamma^{*}$ at $\mathrm{pH} 3.0$, and average residual $\Gamma^{*}$ at $\mathrm{pH}$ 7.0). Deprotonation of biopolymers at this higher $\mathrm{pH}$ can explain this desorption, as it results in electrostatic repulsion between proteins and pectin molecules. In particular, the third to fifth layers initially contained a large amount of adsorbed material, which reduced to $\sim 2 \mathrm{mg} \cdot \mathrm{m}^{-2}$ upon exposure to $\mathrm{pH}$ 7.0. Yet, not all pre-adsorbed material was desorbed, as the residual adsorbed amount at $\mathrm{pH} 7.0$ was still about two-fold greater for three and more preadsorbed layers than for one- and two-layered systems.

These findings are in line with (Rossier-Miranda et al., 2010) who created up to ten-layered interfaces of whey proteins, pectin and protein fibrils, which reduced in adsorbed amount when exposed to neutral $\mathrm{pH}$. Although we could deposit more adsorbed material using greater 
number of layers, they are clearly not effective under intestinal conditions, and are expected to destabilize instantaneously. Whether the layers can resist digestive conditions was tested next for gastric and intestinal conditions.

\section{$\underline{\text { Gastric stability }}$}

Emulsions were incubated in simulated gastric fluid for $2 \mathrm{~h} \mathrm{(pH} \mathrm{3.0,0.5} \mathrm{mg} \mathrm{mL}^{-1}$ pepsin, 150 $\mathrm{mM} \mathrm{NaCl}, 10 \mathrm{mM} \mathrm{CaCl}_{2}, 3{ }^{\circ} \mathrm{C}$ ); Figure 3.5 shows microscopic images during gastric incubation of a primary WPI-stabilized emulsion (W) and a two-layered emulsion (W-P). The size of the primary emulsion droplets increased considerably after 30 min gastric incubation, and it was clear that it underwent coalescence. Digestion of interfacial protein weakens the viscoelastic interfacial structure (Golding et al., 2011; J. MaldonadoValderrama, Terriza, Torcello-Gómez, \& Cabrerizo-Vílchez, 2013) leading to instability rather than the exposure to $\mathrm{pH}$ or salt (Golding et al., 2011). After $2 \mathrm{~h}$ incubation, phase separation was almost complete, i.e., large oil patches floated on top of a gastric fluid that was otherwise almost transparent.

Figure 3.5. Light microscopic images of the indicated emulsions during gastric incubation. The black bar represents $100 \mu \mathrm{m} . \mathrm{W}=$ whey proteins, $\mathrm{P}=$ pectin.

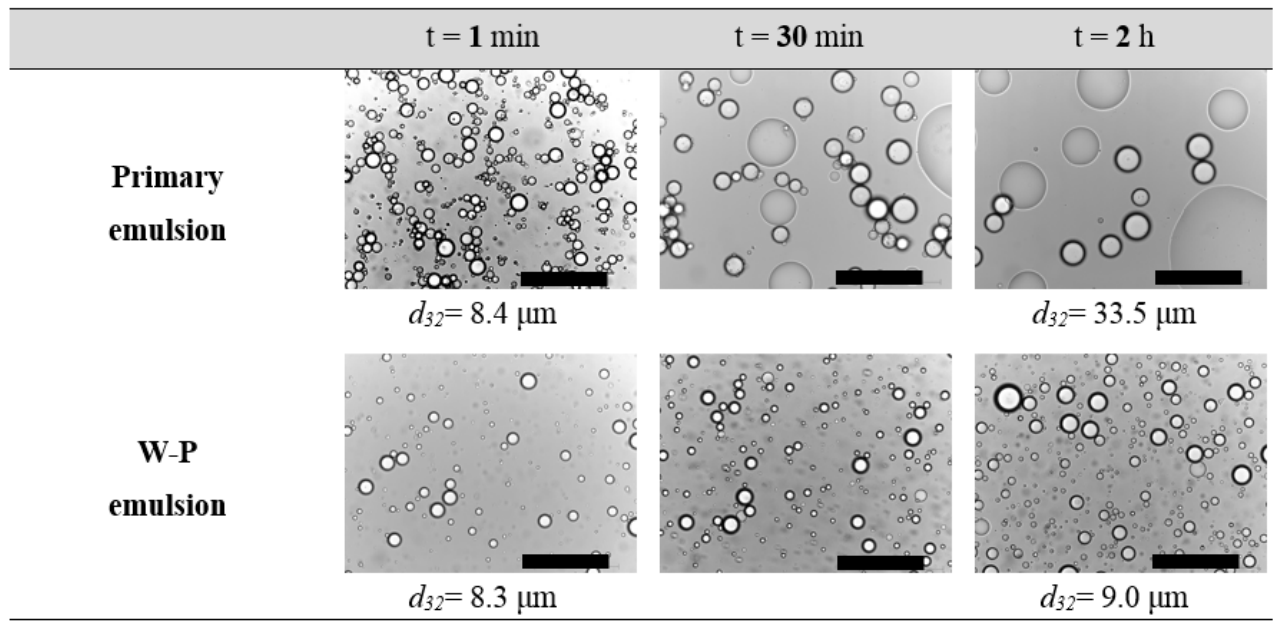

Figure 3.5 shows that the droplet size of two-layered emulsions was hardly affected during $2 \mathrm{~h}$ gastric incubation, so the emulsions were stable against flocculation and coalescence. Apparently, the presence of a pectin layer affected the activity of pepsin, therewith limiting proteolysis and preventing flocculation/coalescence. This is of great importance for the intestinal fate, which will be discussed next. 


\section{Lipolysis -fasted state}

Intestinal lipolysis was studied in vitro under conditions representing the fasted state, including $5 \mathrm{mg} \cdot \mathrm{mL}^{-1}$ bile extract and $0.4 \mathrm{mg} \cdot \mathrm{mL}^{-1}$ lipase (Li, Hu, \& McClements, 2011), and Figure 3.6a shows the results for emulsions with a different number of adsorbed layers. Incubation was conducted for a total time of $150 \mathrm{~min}$, which represents the residence time in the small intestine before reaching the distal small intestine (ileum). The initial rate of lipolysis was the greatest in primary WPI-stabilized emulsions, for which lipolysis was completed ( $\approx 100 \%$ ) after $150 \mathrm{~min}$. This was expected since bile salts can easily displace adsorbed proteins to favour lipase adsorption and action (Golding et al., 2011; Mun, Decker, \& McClements, 2007; Xu et al., 2014).

a.

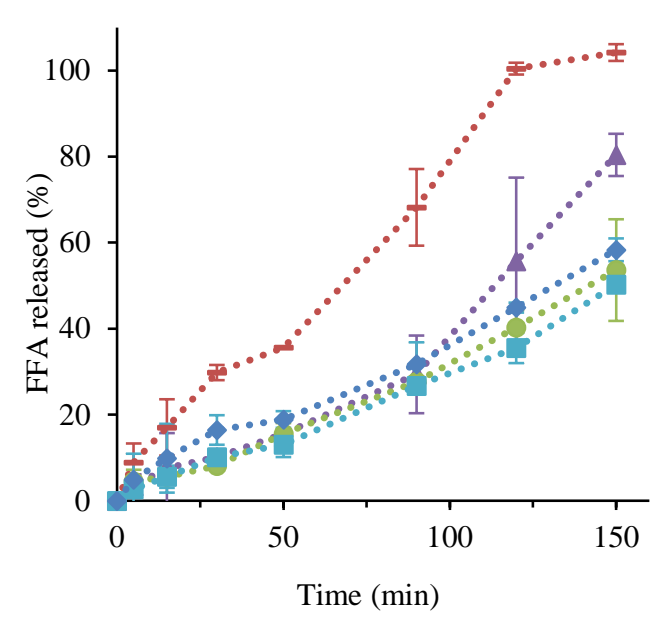

b.

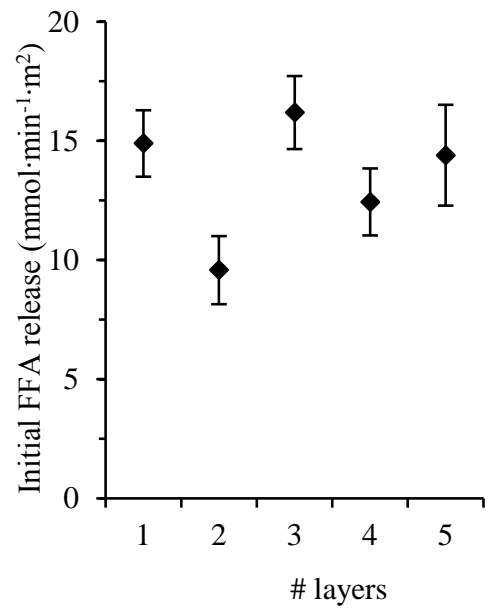

Figure 3.6. a) Free fatty acid (FFA) release from W (-), W-P ( $\Delta$ ), W-P-W (•), W-P-W-P ( $\mathrm{P}-\mathrm{W}(\diamond)$ emulsions, during incubation in simulated intestinal fluid that represents the fasted state (5 $\mathrm{mg} \cdot \mathrm{mL}^{-1}$ bile; $0.4 \mathrm{mg} \cdot \mathrm{mL}^{-1}$ lipase). b) Corresponding initial FFA release rate corrected for available surface area during incubation in simulated intestinal fluid that represents the fasted state $\left(5 \mathrm{mg} \cdot \mathrm{mL}^{-1}\right.$ bile; $0.4 \mathrm{mg} \cdot \mathrm{mL}^{-1}$ lipase $)(n=2)$. $\mathrm{W}=$ whey proteins, $\mathrm{P}=$ pectin.

The extent of FFA release was lower in two-layered emulsions than in primary emulsions; approximately $75 \%$ of the fatty acids were released after $150 \mathrm{~min}$. This indicates that the second layer (pectin) may have protected the interface somewhat and reduced the accessibility of emulsified lipids. This is in line with other studies that found second interfacial layers to lower lipolysis (Klinkesorn \& McClements, 2010; Li et al., 2010; Mun et al., 2006). Please note that this effect was seen with chitosan second layers; pectin layers have not been reported to substantially delay or slow down lipolysis in emulsions (Hu et al., 
2011; Li et al., 2010; Mun et al., 2006; Zeeb, Lopez-Pena, Weiss, \& McClements, 2015). Pectin has even been suggested to promote displacement of other layer materials from the lipid surface (Hu et al., 2011).

Emulsions with three or more layers showed similar FFA release rates as two-layered emulsions during the first 90 min of incubation, but seemed to be more protective against lipolysis in the last 60 min incubation, after which 50\% of the fatty acids were released. No difference in the lipolysis rate and extent was seen between emulsions stabilised by three, four or five layers; which interestingly is in line with the residual interfacial amounts after exposure to $\mathrm{pH}$ 7.0, as measured by reflectometry (Figure 3.4). The residual amount increased with increasing number of interfacial layers up to three, and then levelled out around $2 \mathrm{mg} \cdot \mathrm{m}^{-2}$. However, when taking the droplet size into account, and correcting the lipolysis rate for available interfacial area, their does not seem to be an effect of number of layers (Armand et al., 1999; Golding et al., 2011; Helbig, Silletti, Timmerman, Hamer, \& Gruppen, 2012; Lundin, Golding, \& Wooster, 2008). The initial lipolysis rate per square meter of oil-water interface (Figure 3.6b) was similar for all the emulsions tested, which is in line with other studies (Hu et al., 2011; Lesmes et al., 2010; Tokle et al., 2013). Possibly, the emulsions with a pectin outer layer had a slower initial rate than emulsions with a WPI outer layer, and in literature this has been assigned to interactions of pectin with digestive components (including bile and calcium) (Espinal-Ruiz, Parada-Alfonso, Restrepo-Sánchez, Narváez-Cuenca, \& McClements, 2014a), which can lower accessibility of lipid substrate for lipase and was shown to decrease lipolysis at $0.1 \%$ pectin in solution from $100 \%$ to 85\% FFA release after 2 h, and at $0.2 \%$ to $\sim 70 \%$ FFA release after 2 h (Espinal-Ruiz, ParadaAlfonso, Restrepo-Sánchez, Narváez-Cuenca, \& McClements, 2014b). In the present study, however, this effect is expected to be less strong as the concentration of pectin in the aqueous phase was much lower due to the washing steps.

To conclude, our results showed that pre-adsorbed interfacial layers do not provide much protection against intestinal digestion when expressed per unit of interfacial area, which we could relate to $\mathrm{pH}$-induced desorption. These findings are therefore in line with the fact that the droplet size as such is an important parameter (Armand et al., 1999; Golding et al., 2011; Helbig et al., 2012; Lundin et al., 2008), and is much more relevant than the presence of extra layers.

It is worth mentioning that emulsions made with chitosan were physically unstable and large oil droplets formed when subjected to simulated digestive conditions; therefore, they were digested at a lower rate than fine emulsions, due to the reduction in surface area (Supplementary Figure 3.9). This may explain the contradictory results reported in studies that mention a protective effect of a chitosan outer layer. 


\section{$\underline{\text { Effect of digestive conditions }}$}

Lipolysis was also studied under harsher conditions that simulate the physiological fed state (2.4 mg $\cdot \mathrm{mL}^{-1}$ lipase; $20 \mathrm{mg} \cdot \mathrm{mL}^{-1}$ bile salts), using pure lipase, or a broader range of enzymes (pancreatin). Figure 3.7 shows FFA release for three-layered emulsions (W-P-W), as more layers did not add protectiveness in fasted state conditions. Lipolysis was much faster in the fed state than in the fasted state, showing a steep release in FFAs early in intestinal incubation. Considering the amount of lipase available per unit of interfacial area, this result was not expected; lipase was present in excess amounts already in fasted state conditions (128 mg lipase per $\mathrm{m}^{2}$ interfacial area, while we expect $\sim 2 \mathrm{mg} \cdot \mathrm{m}^{-2}$ to be enough). This suggests that the facilitating effect of bile salts that were also present at a higher concentration (20 $\mathrm{mg} \cdot \mathrm{mL}^{-1}$ instead of $5 \mathrm{mg} \cdot \mathrm{mL}^{-1}$ ), was probably the cause for this effect, which has also been described for other multilayered emulsions (Klinkesorn \& McClements, 2010; Mun et al., 2006).

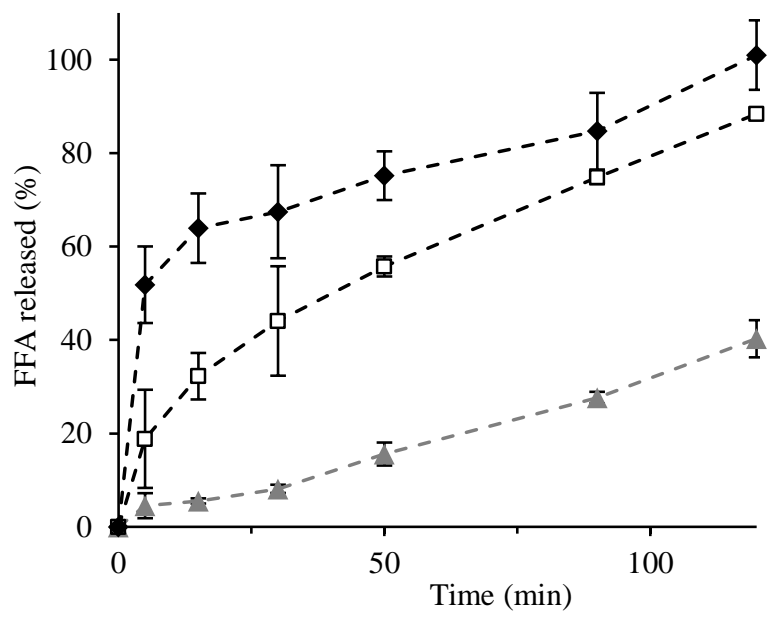

Figure 3.7. Free fatty acid (FFA) release from three-layered W-P-W emulsion during incubation in simulated intestinal fluids representing the fasted state $\left(\Delta ; 5 \mathrm{mg} \cdot \mathrm{mL}^{-1}\right.$ bile; $0.4 \mathrm{mg} \cdot \mathrm{mL}^{-1}$ lipase), fed state ( $\square ; 20 \mathrm{mg} \cdot \mathrm{mL}^{-1}$ bile; $2.4 \mathrm{mg} \cdot \mathrm{mL}^{-1}$ lipase) or fed state with a broader enzyme range $\left(\diamond ; 20 \mathrm{mg} \cdot \mathrm{mL}^{-}\right.$ ${ }^{1}$ bile; $2.4 \mathrm{mg} \cdot \mathrm{mL}^{-1}$ pancreatin $)(n=2)$. $\mathrm{W}=$ whey proteins, $\mathrm{P}=$ pectin.

When the enzyme mixture pancreatin was used instead of pure lipase, lipolysis proceeded even faster and reached a greater final extent, in spite of the fact that pancreatin had 20\% lower activity (tributyrin assay) than the purified lipase. This could be due to the facilitating effect of proteolytic enzymes in pancreatin that can hydrolyse whey proteins in the interface, which increased the accessibility of the emulsified oil for lipase.

The lipolysis profile in intestinal conditions has previously been described to depend more on the physical stability of the emulsion than on properties of the interfacial layer (Zeeb, 
Lopez-Pena, et al., 2015). Our multilayered structures were shown to be physically stable to gastric conditions (Figure 3.5), but when exposed to intestinal components after gastric incubation an oily layer was visible on top of the intestinal fluid (data not shown). This indicated that physical destabilization occurred, which was not due to the $\mathrm{pH}$ shift (Figs. 3 and. 4), but to gastric pepsin action that weakened the multilayered interface, thereby, enhancing subsequent disintegration under intestinal conditions.

To achieve a capsule that releases its content in the distal small intestine, the structural integrity of the layers needs to be enhanced, which can be done through the use of other building blocks: for example, as demonstrated in the work of Rossier-Miranda et al. (2010) who used fibrils and also particles (Rossier-Miranda, Schroën, \& Boom, 2012). Alternatively, inner-layer covalent bonding may make multilayers more resistant to a $\mathrm{pH}$ shift (Zeeb, Gibis, Fischer, \& Weiss, 2012), but has repeatedly been shown to be unable to control lipolysis (Zeeb, Lopez-Pena, et al., 2015; Zeeb, Weiss, \& McClements, 2015). Moreover, intestinal lipolysis could be delayed by inclusion of (multilayered) emulsion droplets in larger hydrogel structures, made of digestible (Dekkers, Kolodziejczyk, Acquistapace, Engmann, \& Wooster, 2016; Sarkar et al., 2015) or indigestible components (Li, Hu, Du, Xiao, \& McClements, 2011; Z. Zhang et al., 2016). Such a strategy would focus on controlling diffusion of enzymes and reaction products, rather than aiming at a barrier effect of the interface.

\subsection{CONCLUSIONS}

Chitosan-containing emulsions showed flocculation under storage conditions and extensive coalescence under physiological $\mathrm{pH}$ conditions, and were not considered suitable. Multilayered WPI/pectin emulsions had improved physical stability compared to primary WPI-stabilized emulsions, during storage and gastric incubation. Adsorption of additional interfacial layers resulted in a great amount of adsorbed material, in particular for whey proteins. Yet, after exposure to $\mathrm{pH}$ 7.0, the residual adsorbed amount was low and similar for three-, four-, and five-layered systems, which resulted in similar initial lipolysis rates. Although in literature multilayered interfaces have sometimes been postulated to affect the rate and extent of lipolysis in emulsions, from the present work it is clear that electrostatically adsorbed multilayers (formed at acid $\mathrm{pH}$ ) readily disintegrate under intestinal conditions and may only be suitable to provide gastric stability and thus for release immediately after the stomach (duodenum). To deliver intact lipids in more distal parts of the digestive tract (ileum), the food structure should be considered at a larger length scale than only interfacial design. 


\section{Acknowledgements}

Research presented in this publication was financially supported by the Graduate School VLAG.

\subsection{SUPPLEMENTARY INFORMATION}

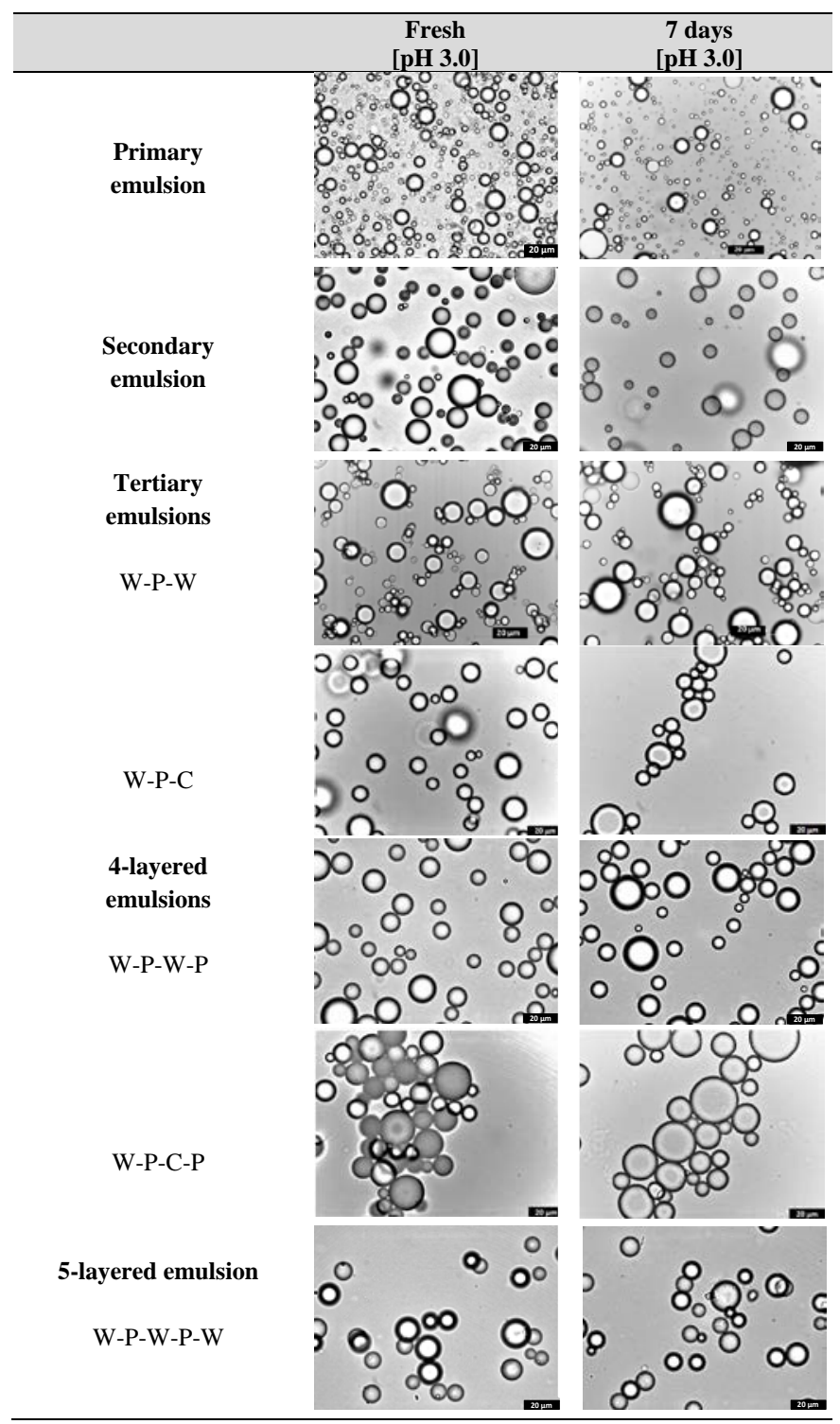

Supplementary Figure 3.8. Light microscopic images of all emulsion systems, fresh and $7 \mathrm{~d}$ after production. The black scale bar represents $20 \mu \mathrm{m}$. W = whey proteins, $\mathrm{P}=$ pectin, $\mathrm{C}=$ chitosan. 


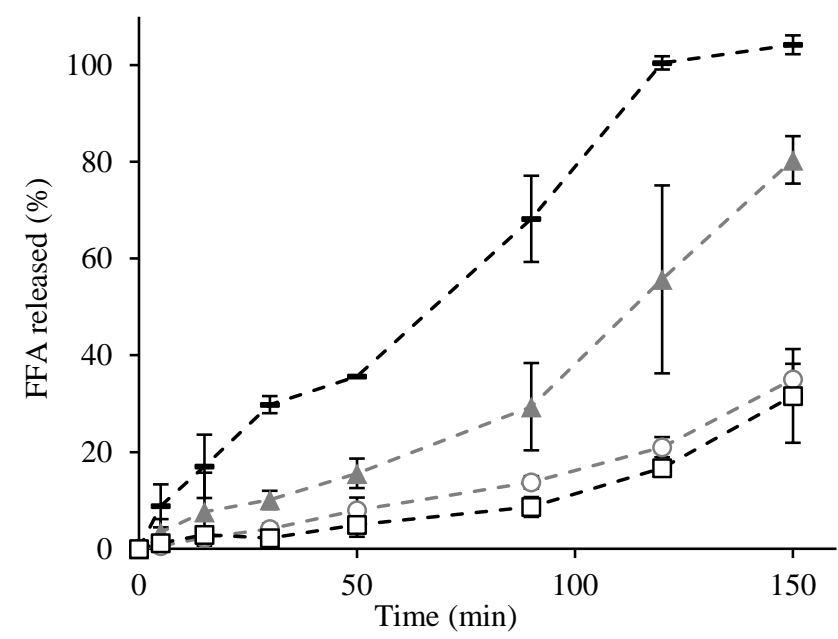

Supplementary Figure 3.9. Free fatty acid release from W (-), W-P ( $\Delta$ ), W-P-C (०), W-P-C-P ( $\square)$ emulsions, during incubation in simulated intestinal fluid that represents the fasted state $(5 \mathrm{mg} / \mathrm{mL}$ bile; $0.4 \mathrm{mg} / \mathrm{mL}$ lipase $)(n=2) . \mathrm{W}=$ whey proteins, $\mathrm{P}=$ pectin, $\mathrm{C}=$ chitosan.

\subsection{REFERENCES}

Acevedo-Fani, A., Salvia-Trujillo, L., Soliva-Fortuny, R., \& Martín-Belloso, O. (2015). Modulating Biopolymer Electrical Charge to Optimize the Assembly of Edible Multilayer Nanofilms by the Layerby-Layer Technique. Biomacromolecules, 16(9), 2895-2903.

Alleleyn, A. M. E., van Avesaat, M., Troost, F. J., \& Masclee, A. A. M. (2016). Gastrointestinal Nutrient Infusion Site and Eating Behavior: Evidence for A Proximal to Distal Gradient within the Small Intestine? Nutrients, 8(3), 117-132.

Aoki, T., Decker, E. A., \& McClements, D. J. (2005). Influence of environmental stresses on stability of O/W emulsions containing droplets stabilized by multilayered membranes produced by a layer-by-layer electrostatic deposition technique. Food Hydrocolloids, 19, 209-220.

Armand, M., Pasquier, B., André, M., Borel, P., Senft, M., Peyrot, J., ... Lairon, D. (1999). Digestion and absorption of 2 fat emulsions with different droplet sizes in the human digestive tract. American Journal of Clinical Nutrition, 70, 1096-1106.

Bakala N'Goma, J.-C., Amara, S., Dridi, K., Jannin, V., \& Carrière, F. (2012). Understanding the lipiddigestion processes in the GI tract before designing lipid-based drug-delivery systems. Therapeutic Delivery, 3(1), 105-124.

Chu, B.-S., Rich, G. T., Ridout, M. J., Faulks, R. M., Wickham, M. S. J., \& Wilde, P. J. (2009). Modulating pancreatic lipase activity with galactolipids: effects of emulsion interfacial composition. Langmuir, 25(16), 9352-9360.

Corstens, M. N., Berton-Carabin, C. C., de Vries, R., Troost, F. J., Masclee, A. A. M., \& Schroën, K. (2017). Food-grade Micro-encapsulation Systems that May Induce Satiety via Delayed Lipolysis: A Review. Critical Reviews in Food Science and Nutrition, 57(10):2218-2244.

Dekkers, B., Kolodziejczyk, E., Acquistapace, S., Engmann, J., \& Wooster, T. J. (2016). Impact of gastric $\mathrm{pH}$ profiles on the proteolytic digestion of mixed $\beta$ lg-Xanthan biopolymer gels. Food \& Function, 7(1), 58-68.

Dickinson, E. (2011). Mixed biopolymers at interfaces: Competitive adsorption and multilayer structures. Food Hydrocolloids, 25(8), 1966-1983.

Dijt, J. C., Stuart, M. A. C., \& Fleer, G. J. (1994). Reflectometry as a tool for adsorption studies. Advances in Colloid and Interface Science, 50, 79-101.

Espinal-Ruiz, M., Parada-Alfonso, F., Restrepo-Sánchez, L. P., Narváez-Cuenca, C. E., \& McClements, D. J. (2014a). Interaction of a dietary fiber (pectin) with gastrointestinal components (bile salts, calcium, 
and lipase): A calorimetry, electrophoresis, and turbidity study. Journal of Agricultural and Food Chemistry, 62(52), 12620-12630.

Espinal-Ruiz, M., Parada-Alfonso, F., Restrepo-Sánchez, L.-P., Narváez-Cuenca, C.-E., \& McClements, D. J. (2014b). Impact of dietary fibers [methyl cellulose, chitosan, and pectin] on digestion of lipids under simulated gastrointestinal conditions. Food \& Function, 5(ii), 3083-3095.

Golding, M., Wooster, T. J., Day, L., Xu, M., Lundin, L., Keogh, J., \& Clifton, P. (2011). Impact of gastric structuring on the lipolysis of emulsified lipids. Soft Matter, 7, 3513-3523.

Guzey, D., \& McClements, D. J. (2006). Formation, stability and properties of multilayer emulsions for application in the food industry. Advances in Colloid and Interface Science, 128(130), 227-248.

Helbig, A., Silletti, E., Timmerman, E., Hamer, R. J., \& Gruppen, H. (2012). In vitro study of intestinal lipolysis using pH-stat and gas chromatography. Food Hydrocolloids, 28(1), 10-19.

Hu, M., Li, Y., Decker, E. A., Xiao, H., \& McClements, D. J. (2011). Impact of Layer Structure on Physical Stability and Lipase Digestibility of Lipid Droplets Coated by Biopolymer Nanolaminated Coatings. Food Biophysics, 6, 37-48.

Hur, S. J., Decker, E. A., \& McClements, D. J. (2009). Influence of initial emulsifier type on microstructural changes occurring in emulsified lipids during in vitro digestion. Food Chemistry, 114, 253-262.

Klinkesorn, U., \& McClements, D. J. (2010). Impact of lipase, bile salts, and polysaccharides on properties and digestibility of tuna oil multilayer emulsions stabilized by lecithin-chitosan. Food Biophysics, 5, 73-81.

Lesmes, U., Baudot, P., \& McClements, D. J. (2010). Impact of interfacial composition on physical stability and in vitro lipase digestibility of triacylglycerol oil droplets coated with lactoferrin and/or caseinate. Journal of Agricultural and Food Chemistry, 58(13), 7962-7969.

Li, Y., Hu, M., Du, Y., Xiao, H., \& McClements, D. J. (2011). Control of lipase digestibility of emulsified lipids by encapsulation within calcium alginate beads. Food Hydrocolloids, 25(1), 122-130.

Li, Y., Hu, M., \& McClements, D. J. (2011). Factors affecting lipase digestibility of emulsified lipids using an in vitro digestion model: Proposal for a standardised pH-stat method. Food Chemistry, 126(2), 498505.

Li, Y., Hu, M., Xiao, H., Du, Y., Decker, E. A., \& McClements, D. J. (2010). Controlling the functional performance of emulsion-based delivery systems using multi-component biopolymer coatings. European Journal of Pharmaceutics and Biopharmaceutics, 76(1), 38-47.

Li, Y., \& McClements, D. J. (2014). Influence of cosurfactant on the behavior of structured emulsions under simulated intestinal lipolysis conditions. Food Hydrocolloids, 40, 96-103.

Lundin, L., Golding, M., \& Wooster, T. J. (2008). Understanding food structure and function in developing food for appetite control. Nutrition and Dietetics, 65(Suppl. 3), S79-85.

Maccarini, M., Himmelhaus, M., Stoycheva, S., \& Grunze, M. (2005). Characterisation and stability of hydrophobic surfaces in water. Applied Surface Science, 252(5), 1941-1946.

Maldonado-Valderrama, J., Terriza, J. A. H., Torcello-Gómez, A., \& Cabrerizo-Vílchez, M. A. (2013). In vitro digestion of interfacial protein structures. Soft Matter, 9(4), 1043-1053.

Maldonado-Valderrama, J., Wilde, P., Macierzanka, A., \& Mackie, A. (2011). The role of bile salts in digestion. Advances in Colloid and Interface Science, 165, 36-46.

Maljaars, P. W. J., Peters, H. P. F., Mela, D. J., \& Masclee, A. A. M. (2008). Ileal brake: a sensible food target for appetite control. A review. Physiology \& Behavior, 95(3), 271-281.

Maljaars, P. W. J., Romeyn, E. A., Haddeman, E., Peters, H. P. F., \& Masclee, A. A. M. (2009). Effect of fat saturation on satiety, hormone release, and food intake. The American Journal of Clinical Nutrition, 89, 1019-1024.

Mao, L., \& Miao, S. (2013). Volatile Release from Whey Protein Isolate - Pectin Multilayer Stabilized Emulsions: Effect of pH, Salt, and Artificial Salivas. Journal of Agricultural and Food Chemistry, 61, 6231-6239.

Marciani, L., Faulks, R., Wickham, M. S. J., Bush, D., Pick, B., Wright, J., ... Spiller, R. C. (2009). Effect of intragastric acid stability of fat emulsions on gastric emptying, plasma lipid profile and postprandial satiety. The British Journal of Nutrition, 101(6), 919-928.

Marciani, L., Wickham, M., Singh, G., Bush, D., Pick, B., Cox, E., ... Spiller, R. C. (2007). Enhancement of intragastric acid stability of a fat emulsion meal delays gastric emptying and increases cholecystokinin release and gallbladder contraction. Am J Physiol Gastrointest Liver Physiol, 292, 1607-1613.

McClements, D. J. (2005). Theoretical analysis of factors affecting the formation and stability of multilayered colloidal dispersions. Langmuir : The ACS Journal of Surfaces and Colloids, 21(14), 9777-9785. 
Minekus, M., Alminger, M., Alvito, P., Ballance, S., Bohn, T., Bourlieu, C., ... Brodkorb, A. (2014). A standardised static in vitro digestion method suitable for food - an international consensus. Food \& Function, 5, 1113-1124.

Mun, S., Decker, E. A., \& McClements, D. J. (2007). Influence of emulsifier type on in vitro digestibility of lipid droplets by pancreatic lipase. Food Research International, 40(6), 770-781.

Mun, S., Decker, E. A., Park, Y., Weiss, J., \& McClements, D. J. (2006). Influence of Interfacial Composition on in vitro Digestibility of Emulsified Lipids: Potential Mechanism for Chitosan's Ability to Inhibit Fat Digestion. Food Biophysics, 1(1), 21-29.

Nazir, A., Schroën, K., \& Boom, R. (2010). Premix emulsification: A review. Journal of Membrane Science, 362(1-2), 1-11.

Rossier-Miranda, F. J., Schroën, K., \& Boom, R. (2010). Mechanical characterization and pH response of fibril-reinforced microcapsules prepared by layer-by-layer adsorption. Langmuir, 26(24), 1910619113.

Rossier-Miranda, F. J., Schroën, K., \& Boom, R. (2012). Microcapsule production by an hybrid colloidosome-layer-by-layer technique. Food Hydrocolloids, 27(1), 119-125.

Sarkar, A., Juan, J.-M., Kolodziejczyk, E., Acquistapace, S., Donato-Capel, L., \& Wooster, T. J. (2015). Impact of protein gel porosity on the digestion of lipid emulsions. Journal of Agricultural and Food Chemistry, 63(40), 8829-8837.

Sarkar, A., Murray, B., Holmes, M., Ettelaie, R., Abdalla, A., \& Yang, X. (2016). In vitro digestion of Pickering emulsions stabilized by soft whey protein microgel particles: influence of thermal treatment. Soft Matter, 12(15), 3558-69.

Sarkar, A., Ye, A., \& Singh, H. (2016). On the role of bile salts in the digestion of emulsified lipids. Food Hydrocolloids, 60, 77-84.

Schönhoff, M. (2003). Layered polyelectrolyte complexes : physics of formation and molecular properties. Journal of Physics: Condensed Matter, 15, 1781-1808.

Tokle, T., Mao, Y., \& McClements, D. J. (2013). Potential biological fate of emulsion-based delivery systems: lipid particles nanolaminated with lactoferrin and $\beta$-lactoglobulin coatings. Pharmaceutical Research, 30(12), 3200-3213.

Tzoumaki, M. V, Moschakis, T., Scholten, E., \& Biliaderis, C. G. (2013). In vitro lipid digestion of chitin nanocrystal stabilized o/w emulsions. Food \& Function, 4(1), 121-9.

van Avesaat, M., Troost, F. J., Ripken, D., Hendriks, H. F., \& Masclee, A. A. M. (2015). Ileal brake activation: macronutrient specific effects on eating behavior? International Journal of Obesity, 39(April), 235243.

Wackerbarth, H., Schön, P., \& Bindrich, U. (2009). Preparation and characterization of multilayer coated microdroplets: Droplet deformation simultaneously probed by atomic force spectroscopy and optical detection. Langmuir, 25(10), 2636-2640.

Wulff-Pérez, M., Vicente, J. De, Martín-Rodríguez, A., \& Gálvez-Ruiz, M. J. (2012). Controlling lipolysis through steric surfactants: New insights on the controlled degradation of submicron emulsions after oral and intravenous administration. International Journal of Pharmaceutics, 423(2), 161-166.

Xu, D., Yuan, F., Gao, Y., Panya, A., McClements, D. J., \& Decker, E. A. (2014). Influence of whey proteinbeet pectin conjugate on the properties and digestibility of $\beta$-carotene emulsion during in vitro digestion. Food Chemistry, 156, 374-379.

Zeeb, B., Gibis, M., Fischer, L., \& Weiss, J. (2012). Crosslinking of interfacial layers in multilayered oil-inwater emulsions using laccase: Characterization and pH-stability. Food Hydrocolloids, 27(1), 126136.

Zeeb, B., Lopez-Pena, C. L., Weiss, J., \& McClements, D. J. (2015). Controlling lipid digestion using enzyme-induced crosslinking of biopolymer interfacial layers in multilayer emulsions. Food Hydrocolloids, 46, 125-133.

Zeeb, B., Weiss, J., \& McClements, D. J. (2015). Electrostatic modulation and enzymatic cross-linking of interfacial layers impacts gastrointestinal fate of multilayer emulsions. Food Chemistry, 180(August), 257-264.

Zhang, Y., \& Zhong, Q. (2015). Multiple-layered coatings on l-glutamine solid microparticles for the retention during storage and enteric delivery during in vitro digestions. Food Hydrocolloids, 43, 584592.

Zhang, Z., Zhang, R., Zou, L., Chen, L., Ahmed, Y., Bishri, W.A. Balamash, K, McClements, D.J. (2016). Encapsulation of curcumin in polysaccharide-based hydrogel beads: Impact of bead type on lipid digestion and curcumin bioaccessibility. Food Hydrocolloids, 58, 160-170. 


\section{Chapter 4}

Interfacial behaviour of

biopolymer multilayers

This chapter has been published as Corstens, M.N., Osorio Caltenco, L.A., de Vries, R., Schroën, K., Berton-Carabin, C.C. (2017) Interfacial behaviour of biopolymer multilayers: influence of in vitro digestive conditions. Colloids and Surfaces B: Biointerfaces 153:199207. 


\begin{abstract}
Although multilayered emulsions have been related to reduced lipolysis, the involved interfacial phenomena have never been studied directly. In this work, we systematically built multilayers of whey protein and pectin, which we further subjected to digestive conditions, using two different techniques: droplet volume tensiometry to investigate interfacial rheology, and reflectometry to determine the amount of adsorbed material. Interfacial tension and dilatational rheology were linked to adsorption/desorption kinetics measured under static in vitro conditions.

The interfacial tension and rheology of the multilayers was rather similar to those found for single whey protein layers, as well as their resistance to duodenal conditions and lipolytic components, which is explained by the rapid destabilisation of multilayers at neutral $\mathrm{pH}$. Sequential adsorption of bile extract or lipase to pre-adsorbed films rapidly lowered the interfacial tension via co-adsorption and displacement, forming a viscoelastic film with low mechanical strength, and highly dynamic adsorption/desorption. When both were present, bile salts dominated the initial adsorption, followed by lipase co-adsorption and formation of lipolysis products that further lowered the interfacial tension, forming a complex interface (including biopolymers, bile salts, lipase, and lipolysis products), independent of preadsorbed biopolymer layers.

Our study shows that the combination of drop volume tensiometry and reflectometry can be used to study complex interfacial behaviours under digestive conditions, which can lead to smart design of interfacial structures for controlled lipolysis in food emulsions.
\end{abstract}

Keywords: Oil-water interface; layer-by-layer; duodenal conditions; droplet volume tensiometry; interfacial rheology; reflectometry. 


\subsection{INTRODUCTION}

Oil-in-water $(\mathrm{O} / \mathrm{W})$ emulsions can conveniently be used as carriers for lipophilic food ingredients (e.g., polyunsaturated lipids, antioxidants, vitamins or flavours) (McClements, 2005). For the delivery of lipophilic food ingredients that are bioactive, it is desirable to control the emulsion's fate in digestive conditions, and notably lipolysis, to ensure that the ingredients are protected and released at a location where they can optimally exert their activity (Verhoeckx et al., 2015). However, this is notoriously difficult because the human gastrointestinal (GI) tract has evolved towards efficient food digestion (Bakala N'Goma, Amara, Dridi, Jannin, \& Carrière, 2012). Lipolysis mostly occurs in the small intestine, due to the presence of bile salts and lipase. To control lipolysis, the oil-water interface plays a pivotal role, as the lipolytic reaction takes place at the surface of oil droplets, where lipase encounters the lipid substrate (i.e., triacylglycerols). Therefore, in order to better engineer $\mathrm{O} / \mathrm{W}$ emulsions as delivery systems, it is crucial to characterise the structure and behaviour of oil-water interfaces under digestive conditions (Corstens et al., 2017a).

Lipase is highly surface active and to preserve its activity, does not unfold after adsorption at oil-water interfaces as many other food proteins do (Reis, Holmberg, et al., 2008; Reis, Holmberg, Watzke, Leser, \& Miller, 2009). Bile salts solubilise digestion products (i.e., free fatty acids and monoacylglycerols) in mixed micelles and, hence, modulate enzyme inhibition (Sarkar, Ye, \& Singh, 2016). They also play multiple roles at the interface to promote lipolysis: They are highly surface-active molecules and efficiently compete for adsorption with proteins (Maldonado-Valderrama et al., 2008; Maldonado-Valderrama, Holgado-Terriza, Torcello-Gómez, \& Cabrerizo-Vílchez, 2013), surfactants (Chu et al., 2010; Torcello-Gómez, Maldonado-Valderrama, de Vicente, et al., 2011; Torcello-Gómez, Maldonado-Valderrama, Martín-Rodríguez, \& McClements, 2011) and gums (Yao et al., 2016). Through orogenic displacement (Euston, Bellstedt, Schillbach, \& Hughes, 2011; Mackie, Gunning, Wilde, \& Morris, 1999; Maldonado-Valderrama et al., 2008), bile salts adsorb at defects in an interfacial biopolymer network, then grow into clusters and compress the network until it falls apart at sufficiently high surface pressures, releasing interfacial biopolymers (Mackie et al., 1999). Hence, bile salts form a highly mobile interface with moderate packing density, not only at bare oil-water interfaces, but also at different preadsorbed viscoelastic films of proteins (Bellesi, Pizones Ruiz-Henestrosa, \& Pilosof, 2014; Wilde \& Chu, 2011), where they further enhance lipolysis by facilitating lipase adsorption (Maldonado-Valderrama, Wilde, Macierzanka, \& Mackie, 2011).

There have been many attempts to inhibit lipolysis in emulsions, especially through interfacial design, often based on sterically hindering lipase from accessing its substrate (Chu et al., 2009; Corstens et al., 2017a; Wulff-Pérez, Vicente, Martín-Rodríguez, \& Gálvez-Ruiz, 2012). Although a few surfactants have been shown to be slightly protective in that respect, 
including digalactosyldiacylglycerols and some Pluronics (Chu et al., 2010; TorcelloGómez, Maldonado-Valderrama, de Vicente, et al., 2011; Torcello-Gómez, MaldonadoValderrama, Jódar-Reyes, \& Foster, 2013), the overall conclusion is that single-layered films (surfactant- and protein-based) cannot prevent lipolysis. Thicker and/or more structurally complex interfaces may provide protection against adsorption of bile components and lipase, for example, specific particles have been reported to reduce lipolysis (Sarkar, Murray, et al., 2016; Tzoumaki, Moschakis, Scholten, \& Biliaderis, 2013), and this has also been reported for specific biopolymer multilayers (Hu, Li, Decker, Xiao, \& McClements, 2011; Klinkesorn \& McClements, 2010; Li et al., 2010; Mun, Decker, Park, Weiss, \& McClements, 2006). Layer-by-layer (LbL) adsorption (based on electrostatic attraction between biopolymers, e.g., proteins and polysaccharides) has been shown to form strong interfacial structures, especially when adsorbed sequentially rather than simultaneously (Ganzevles, Fokkink, van Vliet, Stuart, \& de Jongh, 2008; Ganzevles, Zinoviadou, van Vliet, Stuart, \& de Jongh, 2006; Maldonado-Valderrama, Torcello-Gómez, del Castillo-Santaella, Holgado-Terriza, \& Cabrerizo-Vílchez, 2015). This leads to better storage stability, and coalescence stability in the gastric phase (Corstens et al., 2017b). The claimed effects of biopolymer multilayers to modulate intestinal lipolysis are generally small (Hu et al., 2011; Klinkesorn \& McClements, 2010; Li et al., 2010; Mun et al., 2006) and not systematically confirmed (Klinkesorn \& McClements, 2010; Lesmes, Baudot, \& McClements, 2010; Li et al., 2010; Li \& McClements, 2014; Tokle, Mao, \& McClements, 2013). The contradiction in reported effects may result from small changes in physiological conditions ( $\mathrm{pH}$ and ionic strength) that can affect the interactions between the layers as would be the case in the human digestive system; but no detailed information on the involved interfacial phenomena is available.

Until recently, no appropriate techniques were available to study the interplay between interfacial behaviour and emulsion stability in digestive conditions in depth. Pan and Nitin (2016) used fluorescence resonant energy transfer to study complex emulsions in situ under simulated intestinal digestion conditions, and concluded that bile components (both in the presence and absence of lipase) were able to rapidly disrupt phospholipid-stabilised emulsions (Pan \& Nitin, 2016). Addition of a secondary layer slightly decreased the extent of interface disruption by bile salts and lipase, but did not change the initial rate of displacement and had minor effect on the lipolysis rate, if any, and it was not possible to understand the full behaviour of all lipolytic components at multilayered oil-water interfaces (Pan \& Nitin, 2016). Through the recent introduction of phase exchange in drop techniques, as nicely reviewed by Maldonado-Valderrama et al. (2015), the effect of digestive components on interfacial tension and interfacial rheology can now be studied (del CastilloSantaella et al., 2015). Here we used this approach to systematically investigate the influence of lipolytic duodenal compounds on the behaviour of model biopolymer films prepared by 
the LbL technique (whey protein isolate and pectin) at the oil-water interface, in a droplet volume tensiometer. We determined displacement, co-adsorption and penetration of interfacial components by interfacial tension and rheology measurements, in the presence of bile components, lipase or a combination of them. We further characterised the adsorbed amounts by reflectometry under the same conditions, and linked the interfacial behaviour of complex interfaces to their ability to prevent lipolysis.

\subsection{MATERIAL AND METHODS}

\subsubsection{Materials}

Safflower oil was purchased from De Wit Specialty Oils (19200 Safflower Oil High Linoleic Refined) and used without further purification. Two biopolymers were used: whey protein isolate (WPI) (BiPro, Davisco Food International, Eden Prairie, Minnesota, USA; purity 97.5\%) and pectin (GENU pectin type YM-115-L, DE 72\%, CP Kelco, Atlanta, Georgia, USA). Ethanol (absolute, for analysis) was purchased from Merck (Amsterdam, The Netherlands). Citric acid, sodium citrate tribasic dihydrate, sodium phosphate monobasic, sodium phosphate dibasic, sodium hydroxide, hydrochloric acid, toluene, hexamethyldisilazane (HMDS), porcine bile extract (B8631, crude extract, purity estimated to be 30 to $60 \%$, containing glycine and taurine conjugates of hyodeoxycholic acid and other bile salts according to the supplier) and pancreatin from porcine pancreas (P7545, $8 \times$ USP specification; including trypsin, amylase, lipase, ribonuclease, protease) were purchased from Sigma Aldrich (St. Louis, MO, USA). The lipolytic activity of pancreatin was measured according to Minekus et al. (2014), and found to be $41 \mathrm{U} \cdot \mathrm{mg}^{-1}$ powder. All materials were used directly without further purification. Ultrapure water obtained with a Millipore Milli-Q system (Darmstadt, Germany) was used throughout the study.

\subsubsection{Droplet volume tensiometry}

Both the interfacial tension and rheology were investigated by droplet volume tensiometry using a Tracker device (Teclis, Longessaigne, France), controlled with Windrop 2015 for Windows XP. The optical glass cuvette of $25 \mathrm{~mL}$ (from Hellma-analytics) was equipped with a single-phase exchange device to exchange the continuous phase; when changing from one liquid to the next, the cuvette was rinsed with five times its volume. The cuvette and syringe were temperature-controlled (during multilayer formation $20^{\circ} \mathrm{C}$; during digestive conditions $37^{\circ} \mathrm{C}$ ) using cooling/heating circulator bath (Julabo-CF40). The boundaries of the drop edges were digitised to calculate interfacial tension $(\gamma)$ based on the Young-Laplace equation (Benjamins, Cagna, \& Lucassen-Reynders, 1996).

An oil droplet $(20 \mu \mathrm{L})$ was formed in a protein solution (WPI, $0.1 \mathrm{~g} \mathrm{~L}^{-1}$ in $10 \mathrm{mM}$ citric acid buffer $\mathrm{pH}$ 3.0) and interfacial tension measurements were conducted for at least $3 \mathrm{~h}$ at room 
temperature to allow adsorption to proceed (including protein unfolding and rearrangement). After that, the continuous phase was exchanged with buffer (five times the cuvette volume) to deplete it of non-adsorbed WPI that was present in excess. To build a multilayered film, pectin solution $\left(0.1 \mathrm{~g} \cdot \mathrm{L}^{-1}\right.$ in $10 \mathrm{mM}$ citric acid buffer $\left.\mathrm{pH} 3.0\right)$ was then introduced into the cuvette (10 $\left.\mathrm{mL} \cdot \mathrm{min}^{-1}, 15 \mathrm{~min}\right)$ and allowed to adsorb as a second layer for 15 more min, followed by a buffer-exchange (10 mM citric acid buffer pH 3.0, $15 \mathrm{~min}$ ). The next layers were adsorbed using the same steps, alternating WPI and pectin, until up to four layers were formed. After that, the bare oil-water interface, single-layered surface and multilayered surfaces were exposed to $10 \mathrm{mM}$ phosphate buffer $\mathrm{pH} 7.0$ first, followed by $0.5 \mathrm{~g} \mathrm{~L}^{-1}$ bile and $0.16 \mathrm{~g} \mathrm{~L}^{-1}$ lipase for $30 \mathrm{~min}$, individually or simultaneously. The ratio of bile and lipase was chosen for physiological relevance (Maldonado-Valderrama et al., 2013).

Dilatational rheology was measured for single- and multilayered interfacial films, after equilibration at $\mathrm{pH} 3.0\left(20^{\circ} \mathrm{C}\right)$, and in duodenal conditions $\left(\mathrm{pH} 7.0,37^{\circ} \mathrm{C}\right)$. Oscillatory perturbation of the area of the oil drop was carried out with a motor-driven syringe at a frequency of $0.05 \mathrm{~Hz}$, and with amplitude sweeps $\left(\Delta A / A_{0}\right)$ of $1 \%, 5 \%, 10 \%$, and $12 \%$ (5 active and 5 passive cycles per amplitude). After exposing the films to lipolytic components, oscillations were performed as well, but only with amplitudes $\left(\triangle A / A_{0}\right)$ of $1 \%, 5 \%, 8 \%$ and $10 \%$ because higher amplitudes led to droplet detachment. The surface dilatational elastic and viscous moduli ( $E$ ' ${ }_{\mathrm{d}}$ and $E$ ”, , respectively) were determined from the measured dynamic interfacial tension response, taking the intensity and phase of the first harmonic after Fourier transformation of the $\gamma$ signal. Moreover, the response was visualised directly in so-called Lissajous plots: surface pressure (stress $\pi=\gamma_{0}-\gamma$, with $\gamma_{0}$ the interfacial tension before deformation) against deformation (strain $\Delta A / A_{0}=\left(A_{0}-A\right) / A_{0}$, where $A_{0}$ is the interfacial area before deformation and $A$ is the interfacial area during oscillation). The shape of this plot gives direct indications regarding the behaviour of the interfacial film in dilatational deformation: a straight line, circle or elliptic shape correspond to an elastic, viscous or viscoelastic response, respectively (Deshpande, Murali Krishnan, \& Sunil Kumar, 2010; Sagis \& Scholten, 2014). In addition, a nonlinear behaviour of the oil-water interface results in asymmetric Lissajous plots: for instance, asymmetry may reveal softening in expansion (the upper right part of the plot) or hardening in compression (the lower left part of the plot).

\subsubsection{Reflectometry}

Adsorption and desorption of lipolytic components at biopolymer-coated hydrophobic surfaces was investigated using fixed angle stagnation point reflectometry, which was performed on a homebuilt apparatus (Dijt, Stuart, \& Fleer, 1994). The method was the same as described previously (Corstens et al., 2017b). In short, we used hydrophobised silicon surfaces (polished silicon wafer, diameter $150 \mathrm{~mm}$; Siltronic, München, Germany) to 
represent safflower oil, similar to (Schröen, Cohen Stuart, Van der Padt, \& Van't Riet, 1994; Schroen, Stuart, Maarschalk, Vanderpadt, \& Vantriet, 1995). The reflectometry signal $S$ was monitored in time while flushing various solutions over the hydrophobic surface that was covered by specific numbers of layers, at a speed of $2 \mathrm{~mL} \cdot \mathrm{min}^{-1}$, and translated into an adsorbed amount (defined as $\Gamma^{*}\left(\mathrm{mg} \cdot \mathrm{m}^{-2}\right)$ ), using an estimated $Q$-factor of 21.5 as described previously (Corstens et al., 2017b). First, whey protein was adsorbed $\left(0.5 \mathrm{~g} \cdot \mathrm{L}^{-1} \mathrm{WPI}\right.$ in $10 \mathrm{mM}$ citric acid buffer $\mathrm{pH}$ 3.0; $15 \mathrm{~min}$ ), and next the sample was washed with buffer (10 $\mathrm{mM}$ citric acid buffer $\mathrm{pH} 3.0 ; 15 \mathrm{~min}$ ) to remove loosely attached components. The formed film was exposed to duodenal $\mathrm{pH}(10 \mathrm{mM}$ phosphate buffer $\mathrm{pH} 7.0 ; 15 \mathrm{~min})$ and lipolytic components $\left(10 \mathrm{mM}\right.$ phosphate buffer $\mathrm{pH} 7.0$ containing $0.25 \mathrm{~g} \cdot \mathrm{L}^{-1}$ bile, $0.25 \mathrm{~g} \cdot \mathrm{L}^{-1}$ lipase, or both at $0.25 \mathrm{~g} \cdot \mathrm{L}^{-1}$ each; $\left.30 \mathrm{~min}\right)$. Finally, the film was rinsed again with buffer $(10 \mathrm{mM}$ phosphate buffer at $\mathrm{pH} 7.0 ; 15 \mathrm{~min}$ ).

\subsubsection{Experimental design}

All reflectometry, interfacial tension and rheology experiments were performed at least twice as independent replicates (for bile, lipase, and a combination of both). This implies that formation and behaviour of single- and four-layered films, including exposure to $\mathrm{pH}$ 7.0, was performed in six independent replicates in the droplet volume tensiometer. Analysis of variance (ANOVA) was performed using the statistical software package SPSS Version 23.0.0.2 (IBM ${ }^{\odot}$ SPSS Statistics, Chicago, IL, USA). For significant ANOVA outcomes, means were compared pairwise $(p<0.05)$ using post hoc Tukey HSD. From the oscillation experiments, the middle 3 cycles per amplitude were used for analysis of the rheological moduli.

\subsection{RESULTS AND DISCUSSION}

Reflectometry and droplet volume tensiometry were used to systematically investigate the effect of conditions corresponding to the duodenum ( $\mathrm{pH} 7.0,37^{\circ} \mathrm{C}$, bile and lipase) on the interfacial behaviour of multilayered whey protein-pectin films, as reflected in their interfacial tension, dilatational rheology, and adsorbed amount.

\subsubsection{Behaviour of multilayered films at the oil-water interface}

Formation and viscoelasticity of multilayers at $\mathrm{pH} 3.0$

Layers of whey protein isolate (WPI, positively charged at $\mathrm{pH}$ 3.0) and pectin (negatively charged at $\mathrm{pH}$ 3.0) were sequentially deposited on a single oil-in-water droplet using an automated drop tensiometer. Figure 4.1 shows the interfacial tension with one to four adsorbed biopolymer layers. The first WPI layer lowered the interfacial tension to $12.9 \pm 0.6$ $\mathrm{mN} \cdot \mathrm{m}^{-1}$ after $3 \mathrm{~h}$ of adsorption, which is a typical profile for globular proteins at vegetable 


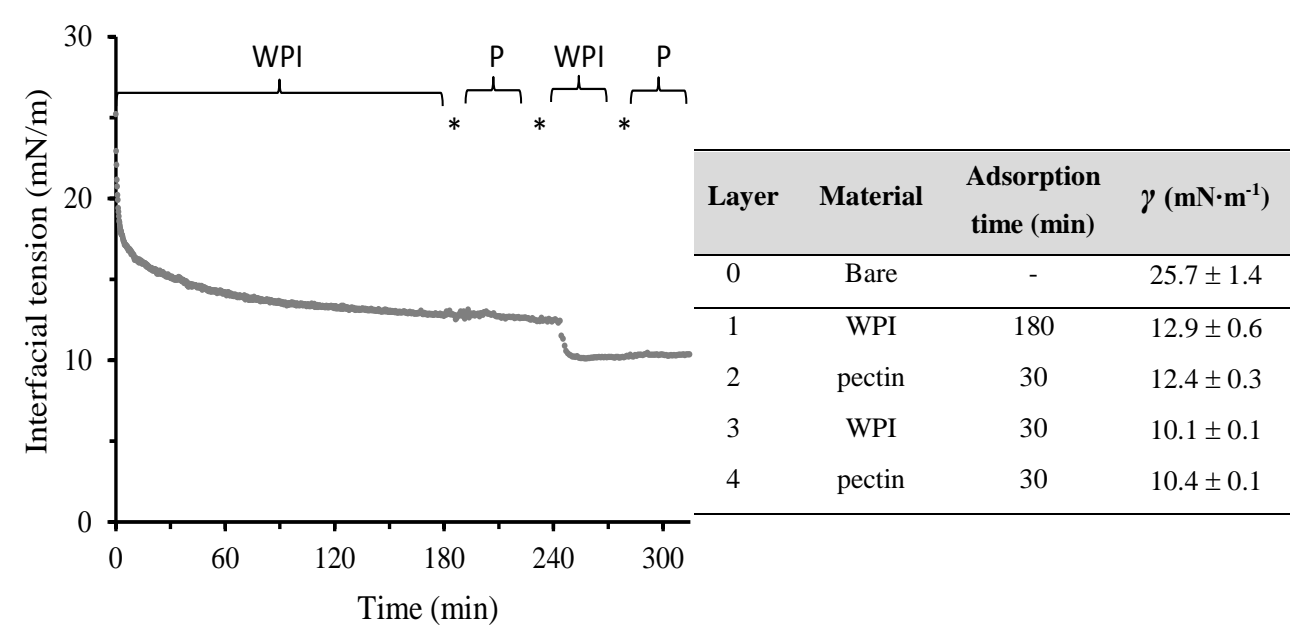

Figure 4.1. Interfacial tension $(\gamma)$ as function of the number of adsorbed WPI $\left(0.1 \mathrm{~g} \cdot \mathrm{L}^{-1}\right)$ and pectin $(0.1$ $\mathrm{g} \cdot \mathrm{L}^{-1}$ ) layers at the oil-water interface, $10 \mathrm{mM}$ citric acid buffer $\mathrm{pH}$ 3.0. Figure (left) shows the adsorption in time (* indicates a 15 min buffer rinse), and table (right) average values represent mean \pm standard deviation $(n \geq 6)$.

oil-water interfaces (Benjamins, Lyklema, \& Lucassen-Reynders, 2006; MaldonadoValderrama et al., 2008, 2013; Maldonado-Valderrama, Miller, Fainerman, Wilde, \& Morris, 2010), and can be ascribed to adsorption - unfolding - rearrangement of proteins at the oilwater interface. During the rinsing step, the interfacial tension remained stable, indicating minimal desorption, and thus irreversible adsorption, as expected for globular proteins (Maldonado-Valderrama, Gálvez-Ruiz, Martín-Rodríguez, \& Cabrerizo-Vílchez, 2004; Miller et al., 2005).

Adsorption of a secondary pectin layer (indicated by P) had negligible effect on the interfacial tension (Figure 4.1). Since pectin itself has been shown to considerably lower the interfacial tension (Schmidt, Schütz, \& Schuchmann, 2016; Yao et al., 2016), this implies that even though pectin adsorbed on top of the whey protein layer through electrostatic attraction, it did not interact with the oil-water interface to reduce interfacial tension (Ganzevles et al., 2008). Adsorbing a third layer of whey protein further reduced the interfacial tension slightly (on average $2.3 \mathrm{mN} \cdot \mathrm{m}^{-1}$ ). This indicates that additional protein molecules somewhat influenced the interfacial structure, either through direct contact with the interface or indirectly via an effect on the pectin layer that in turn may have influenced the first whey protein layer. The initial adsorption rate of the third layer was lower than of the first layer of whey protein, and the extent of interfacial tension reduction was less (see Figure 4.1). Both effects were expected; the formation of the primary layer included protein unfolding and rearrangement, while the formation of the third layer was mainly driven mainly by 
electrostatic attraction, which is expected to play a less dominant role in the adsorption process (Schönhoff, 2003). Finally, a fourth layer of pectin did not further influence the interfacial tension, as was the case for the second layer. Still, adsorption of corresponding layers was previously shown to account for $\sim 0.5 \mathrm{mg} \cdot \mathrm{m}^{-2}$ (second pectin layer), $\sim 2 \mathrm{mg} \cdot \mathrm{m}^{-2}$ (third WPI layer), and $\sim 1 \mathrm{mg} \cdot \mathrm{m}^{-2}$ (fourth pectin layer) (Corstens et al., 2017b).

To study the mechanical properties of the interfacial films, dilatational rheology measurements were performed, and elastic and viscous moduli were calculated (Figure 4.2). For single protein films, the elastic modulus was much higher than the viscous modulus, indicative of an elastic character, which was also reported for various protein films (Bos \& Vliet, 2001; Sagis \& Scholten, 2014). The four-layered film showed higher elastic moduli compared to a single protein film, and extremely low viscous moduli. In all cases, the moduli depended only mildly on deformation. Please note that the moduli need to be treated with care since gel formation and viscous forces may affect them as well (Rühs, Scheuble, Windhab, \& Fischer, 2013).
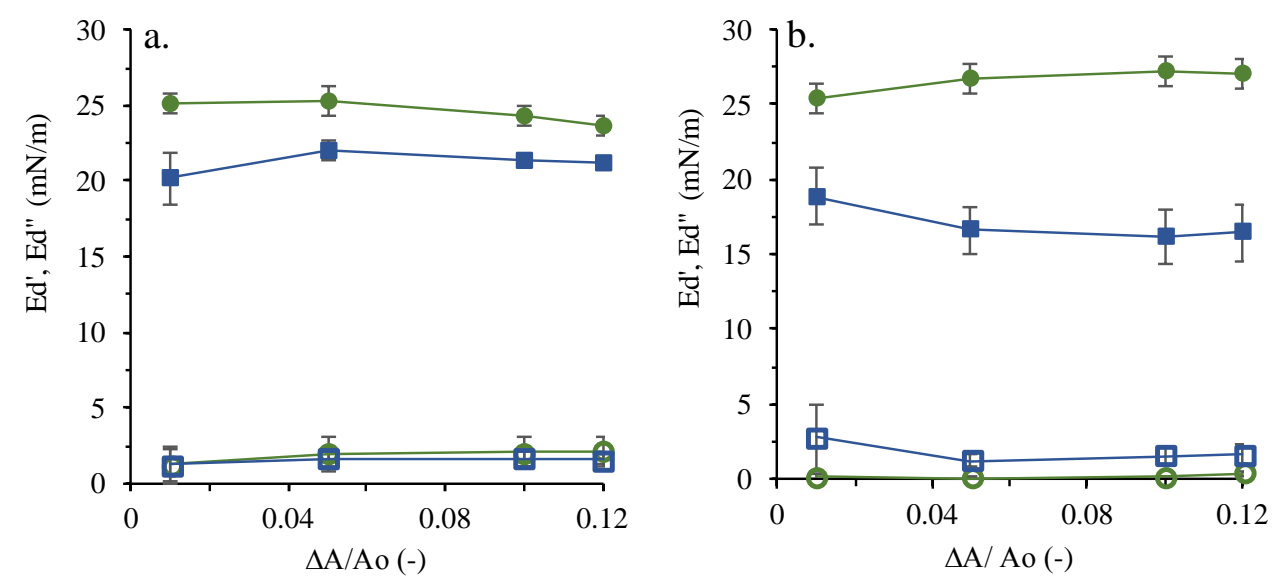

Figure 4.2. Dilatational elastic ( $E_{d}$ ' closed symbols) and viscous ( $E_{d}$ " open symbols) moduli as a function of the applied deformation. Comparing interfacial behaviour of a) single protein film and b) four-layered film alternating WPI and pectin at $\mathrm{pH} 3.0, T=20^{\circ} \mathrm{C}$ (green-circles) and at $\mathrm{pH} 7.0, T=37$ ${ }^{\circ} \mathrm{C}$ (blue-squares).

To further explore any nonlinear interfacial behaviour during oscillatory cycles, so-called Lissajous plots are relevant (Lucassen-Reynders, Benjamins, \& Fainerman, 2010; Rühs et al., 2013; Sagis \& Fischer, 2014; Sagis \& Scholten, 2014). The plots for single whey protein films showed a narrow elliptic shape indicating a predominantly elastic behaviour of the oilwater interface (Figure 4.3a), which is in line with the high elastic moduli measured. In both compression and expansion $\left(\triangle A / A_{0}=0.12\right.$ ), asymmetries in the plot could be seen: the slopes of the secant lines were lower than the slopes of the tangent lines (Sagis \& Fischer, 2014). 
This means that in the extremes of compression and expansion the response was less elastic (strain softening), indicating that the protein network was somehow disconnected. At lower deformations (see Supplementary Figure 4.6) such strain softening was not observed, which implies that substantial deformation of the interface is required for disruption of the network.

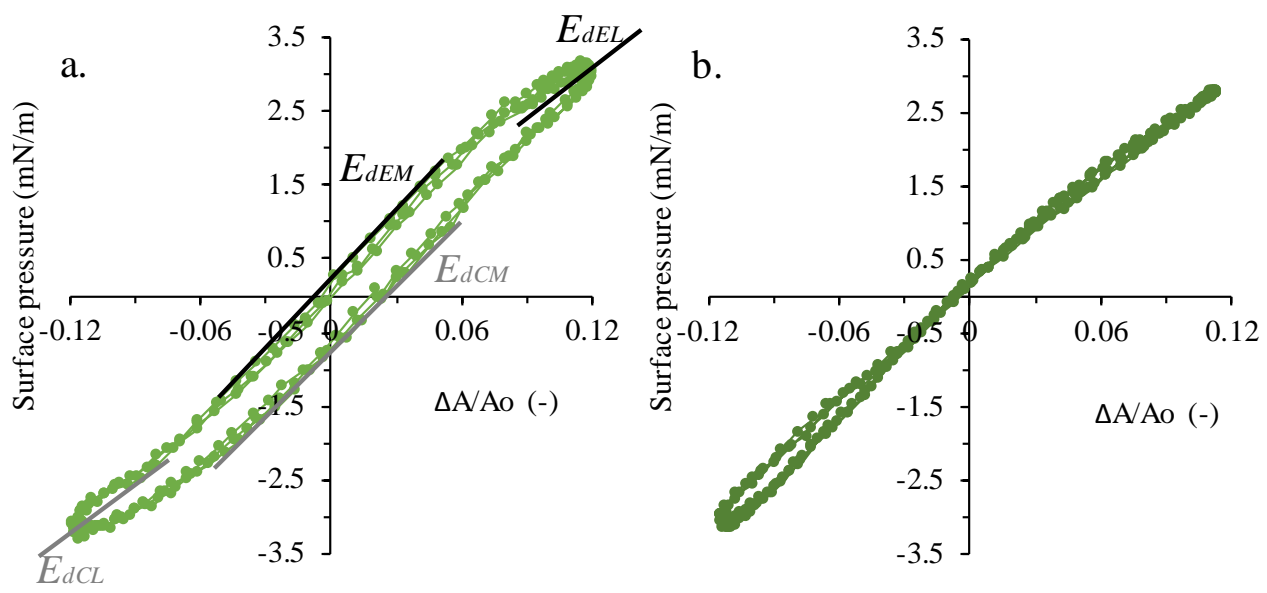

Figure 4.3. Lissajous plot of surface pressure versus deformation (up to 12\%) for a) single protein film, and b) four-layered film, $T=20^{\circ} \mathrm{C}$, pH 3.0, $10 \mathrm{mM}$ citric acid buffer. For clarity, only one replicate is shown for each tested condition, but similar plots were obtained with more replicates $(n=3)$. In panel A the lines at minimum or large extension ( $E_{d E M}, E_{d E L}$, respectively); or at minimum or large compression ( $\mathrm{E}_{d C M}, \mathrm{E}_{d C L}$, respectively) are shown.

The plots for four-layered interfacial films (Figure 4.3b) showed an almost straight profile, indicating almost purely elastic behaviour, which again is in line with the high elastic moduli. Non-linear behaviour was observed only in expansion at high deformations: the slope of the secant line was slightly lower than that of the tangent line, revealing slight strain softening. Hence, the multilayered structure moderately disconnected laterally, but only at large deformations (see Supplementary Figure 4.6, for the response at lower strains). Also in compression, a linear elastic response of multilayered structure was observed even at large area reduction $\left(\triangle A / A_{0}=0.12\right)$, which is in contrast to our findings for single protein films.

It is well known that within multilayered interfaces, interactions between the different layers lead to increased film thickness (Jourdain, Schmitt, Leser, Murray, \& Dickinson, 2009) and mechanical strength (Rossier-Miranda, Schroën, \& Boom, 2010) compared to single layered interfaces. However, similar surface pressures were obtained in compression and expansion for both single- and four-layered films. Therefore, it can be concluded that the presence of polysaccharides did not influence the mechanical strength of the interfacial film, and that mechanical strength is a result of multiple and strong interactions between proteins in the 
first layer (Ganzevles, Kosters, van Vliet, Stuart, \& de Jongh, 2007; Sagis \& Scholten, 2014). On the other hand, the presence of polysaccharides did slightly increase the film elasticity and decreased the tendency to collapse at large deformations (less strain softening in compression).

\section{Behaviour of multilayers in simulated duodenal conditions}

Lipolysis mainly takes place in the duodenum, which is the focal point of this paper, and therefore, the films were exposed to $\mathrm{pH} 7.0$ and a temperature of $37^{\circ} \mathrm{C}$. Table 4.1 shows the interfacial tension of single- and four-layered adsorbed films at the oil-water interface after production ( $\mathrm{pH} 3.0,20^{\circ} \mathrm{C}$ ), after switching to $\mathrm{pH} 7.0\left(20^{\circ} \mathrm{C}\right)$ and after applying body temperature $\left(37^{\circ} \mathrm{C}\right)$. For the single protein film, the results from the statistical analysis showed no influence of duodenal conditions ( $\mathrm{pH}$ and temperature) on the interfacial tension ( $p=0.059$ ), indicating that proteins remained adsorbed and were not subjected to spatial rearrangement at the oil-water interface. For the four-layered film, statistical analysis showed an effect of duodenal conditions ( $\mathrm{pH}$ and temperature) on the interfacial tension $(p=0.000)$. The interfacial tension increased while switching from $\mathrm{pH} 3.0$ to $\mathrm{pH} 7.0(p=0.000)$, independent of the temperature ( $p=0.395$ ), which indicates rearrangements in the oil-water interface at neutral $\mathrm{pH}$, which we attribute to desorption of biopolymers (Corstens et al., 2017b).

Regarding the effect of the number of layers, the interfacial tension in the presence of fourlayered films was lower compared to that with single-layered films $(p=0.000)$ only after production ( $\mathrm{pH} 3.0,20^{\circ} \mathrm{C}$ ); no difference between both interfaces was seen anymore after the $\mathrm{pH}$ was switched to $\mathrm{pH} 7.0$ at $20^{\circ} \mathrm{C}(p=0.18)$ or at $37^{\circ} \mathrm{C}(p=0.20)$.

Table 4.1. Comparison of interfacial tension at $\mathrm{pH} 3.0,20^{\circ} \mathrm{C}$ and $7.0,20^{\circ} \mathrm{C}$ or $7.0,37^{\circ} \mathrm{C}$.

\begin{tabular}{cccc}
\hline \# layers & $\gamma_{\mathbf{p H ~ 3 . 0 , ~ 2 0}}{ }^{\circ} \mathbf{C}\left(\mathbf{m N} \cdot \mathbf{m}^{-\mathbf{1}}\right)$ & $\gamma_{\mathbf{p H ~}} \mathbf{7 . 0 , 2 0}{ }^{\circ} \mathbf{C}\left(\mathbf{m N} \cdot \mathbf{m}^{-\mathbf{1}}\right)$ & $\gamma_{\mathbf{p H ~}}$.0, 37 ${ }^{\circ} \mathbf{C}\left(\mathbf{m N} \cdot \mathbf{m}^{-\mathbf{1}}\right)$ \\
\hline 1 & $12.9 \pm 0.6$ & $12.2 \pm 0.3$ & $12.2 \pm 0.1$ \\
4 & $10.4 \pm 0.1$ & $11.6 \pm 0.3$ & $11.9 \pm 0.3$
\end{tabular}

Values are obtained as mean \pm standard deviation $(n \geq 2)$. 
To study the mechanical properties of the interfacial films under duodenal conditions, dilatational rheology measurements were performed (Figure 4.2). Compared to the production conditions ( $\mathrm{pH} 3.0,20^{\circ} \mathrm{C}$ ), the dilatational elastic moduli decreased for both films after exposure to duodenal conditions ( $\mathrm{pH} 7.0,37^{\circ} \mathrm{C}$ ), which indicates a decrease in film stability. The four-layered film (Figure 4.2b) was more affected by these conditions than a single protein film (Figure 4.2a), resulting in an even lower elasticity.

The Lissajous plots in Table 4.2 directly visualise the response of the oil-water interface to dilatational deformation. When the $\mathrm{pH}$ was increased to 7.0 , the maximum surface pressure decreased both in compression and in expansion compared to the same film at pH 3.0 (see Supplementary Figure 4.6, for all Lissajous plots). This can happen if interfacial components rapidly adsorb and desorb during the oscillation cycles, thereby compensating for the change in surface pressure induced by the change in interfacial area. Besides, the plots became slightly wider when switching to $\mathrm{pH}$ 7.0, suggesting less elastic behaviour of the oil-water interface, which can reveal a reduction in intermolecular interactions between adsorbed biopolymers. We hypothesise that such a decrease in the connectivity of the interfacial network could be due to loss of interfacial "patches" (i.e., protein-polysaccharide complexes initially formed by charge interaction) induced by the $\mathrm{pH}$ change. This is in line with previous data obtained with reflectometry, in which we found that much more material desorbed from biopolymer multilayers exposed to $\mathrm{pH} 7.0$ compared to a single protein layer, indicating that protein-polysaccharide complexes are more prone to $\mathrm{pH}$-induced desorption than the individual protein components (Corstens et al., 2017b). 
Table 4.2. Lissajous plots of surface pressure versus deformation (up to 5\%) for single protein film, and four-layered film, after formation ( $T=20^{\circ} \mathrm{C}$, $\mathrm{pH} 3.0$ ), under duodenal conditions $\left(T=37^{\circ} \mathrm{C}, \mathrm{pH} 7.0\right)$, after exposing to lipase $\left(0.16 \mathrm{~g} \cdot \mathrm{L}^{-1}, \mathrm{pH} 7.0, T=37^{\circ} \mathrm{C}\right)$ and after exposing to bile extract $\left(0.5 \mathrm{~g} \cdot \mathrm{L}^{-1}\right.$, $\mathrm{pH} 7.0$, $T=37^{\circ} \mathrm{C}$ ). For clarity, only one replicate is shown for each tested condition, but similar plots were obtained with more replicates $(n \geq 2)$.

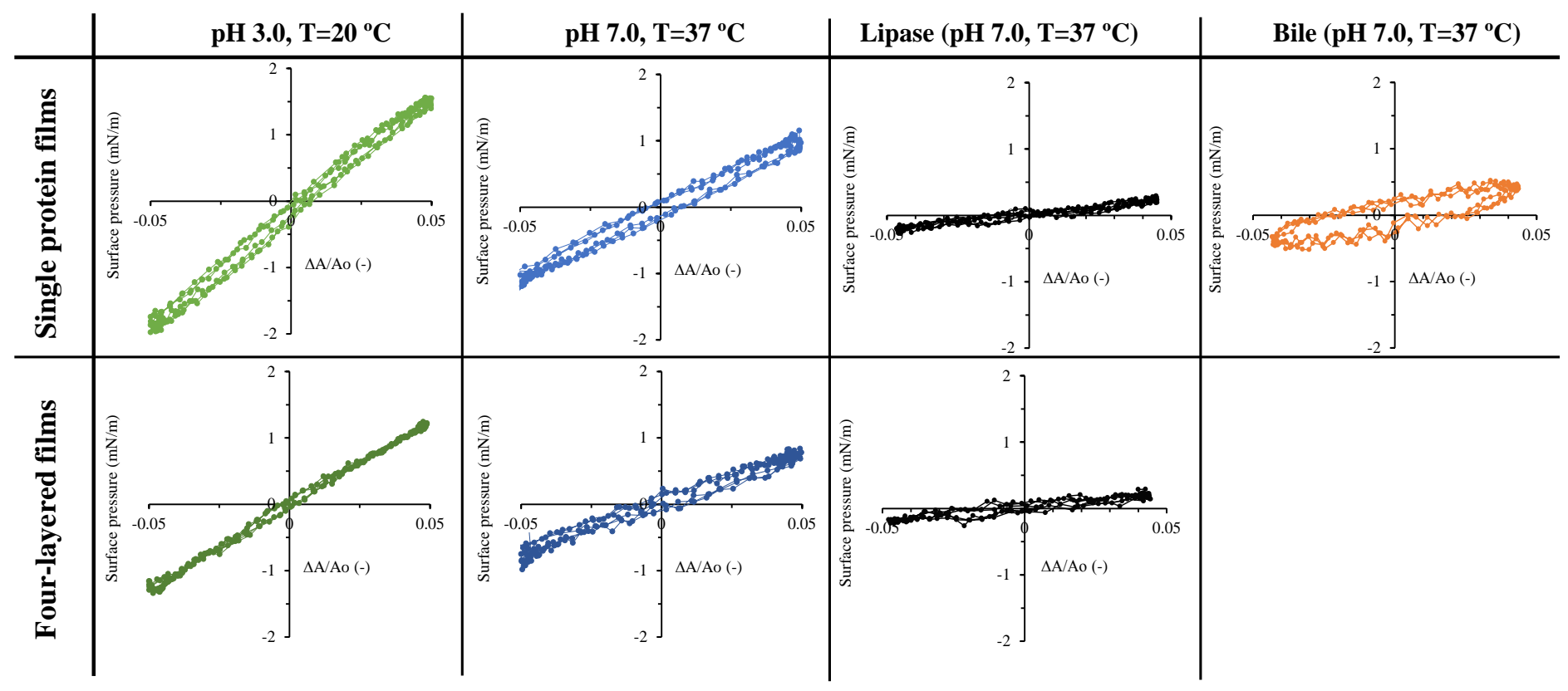


Influence of lipolytic components on film properties and stability

When exposing oil-water interfaces with various numbers of adsorbed biopolymer layers to bile extract and lipase, individually or simultaneously, different effects on interfacial tension were observed. Figure 4.4a shows the response for the bare surface, Figure 4.4B for a single whey protein film and Figure 4.4c for a four-layered film. The effect on interfacial dilatational rheology is shown in Table 4.2.

\section{Bile extract}

Adsorption of bile extract $\left(0.5 \mathrm{~g} \cdot \mathrm{L}^{-1}\right)$ at a bare safflower oil-water interface rapidly lowered the interfacial tension (Figure 4.4a, light orange line) to a saturation value of $9.5 \pm$ $0.4 \mathrm{mN} \cdot \mathrm{m}^{-1}$ within $25 \mathrm{~min}$, which is in line with literature data $\left(8.0-12.2 \mathrm{mN} \cdot \mathrm{m}^{-1}\right.$; on vegetable oil-water interfaces) (Maldonado-Valderrama et al., 2013; Torcello-Gómez, Maldonado-Valderrama, de Vicente, et al., 2011; Torcello-Gómez, Maldonado-Valderrama, Martín-Rodríguez, et al., 2011). When bile was introduced after biopolymer layers were initially adsorbed and equilibrated, similar phenomena occurred depending on the initial number of biopolymer layers (one, Figure 4.4b or four, Figure 4.4c). The interfacial tension reduced further to $\sim 8.6 \mathrm{mN} \cdot \mathrm{m}^{-1}$, at a similar interfacial tension reduction rate of -23 $\mu \mathrm{N} \cdot \mathrm{m}^{-1} \cdot \mathrm{s}^{-1}$ or $-26 \mu \mathrm{N} \cdot \mathrm{m}^{-1} \cdot \mathrm{s}^{-1}$, for one and four layers respectively. This indicates that bile extract adsorbs onto the biopolymer film, therewith displacing part of the pre-adsorbed interfacial material (Euston et al., 2011; Mackie et al., 1999; Maldonado-Valderrama et al., 2008). The overall effect on interfacial tension seems to be greater than for a bare surface, which suggests favourable molecular interactions between bile and biopolymers at the oilwater interface (Bellesi et al., 2014).

Fifteen min after bile salts were introduced in the continuous phase, dilatational deformation of the oil drop was applied and the surface pressure response was measured. The presence of bile largely reduced the elasticity of the interfacial film independent of deformation ( $E_{\mathrm{d}}{ }_{\mathrm{d}}$ of $8.5 \pm 0.3 \mathrm{mN} \cdot \mathrm{m}^{-1}$; Supplementary Figure 4.7). For single whey protein films (Table 4.2, top row), exposure to bile made the interface become highly viscous compared to the original behaviour at $\mathrm{pH} 3.0$ and 7.0, showing that a cohesive protein network was no longer present at the interface. In addition, the net maximum surface pressure upon compression and expansion was lowered in the presence of bile, which indicates that bile salt molecules are able to exchange rapidly between bulk and interface (Bellesi et al., 2014). The dilatational response of the four-layered structure after exposure to bile could not be measured because the drop detached, but it is expected to be at most similar to that found for the single layer, but most probably even more dominated by bile components, given the synergistic desorption mentioned earlier. Interfacial rheology measurements could also not be performed at the bare 
oil-water interface in the presence of bile; the drop detached because of the very low interfacial tension.
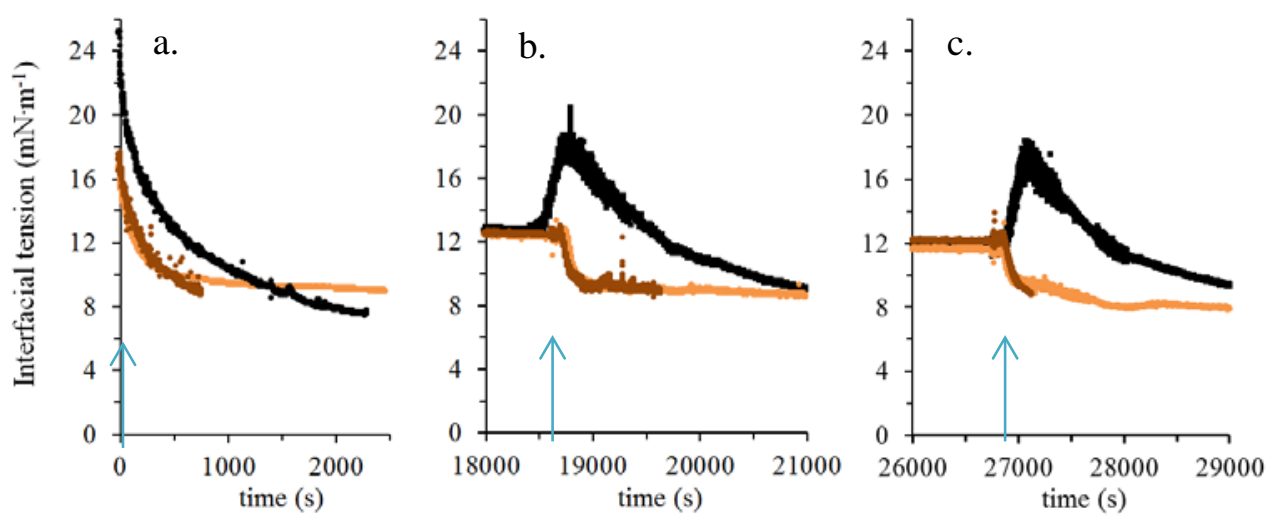

Figure 4.4. Adsorption kinetics of lipolytic components at a) bare safflower oil-water interface, or with a pre-formed b) protein film or c) four-layered film. The orange lines represent bile extract $\left(0.5 \mathrm{~g} \cdot \mathrm{L}^{-1}\right)$, the black lines pancreatic lipase $\left(0.16 \mathrm{~g} \cdot \mathrm{L}^{-1}\right)$, and the brown line the mixture $\left(0.5 \mathrm{~g} \cdot \mathrm{L}^{-1}\right.$ bile and $0.16 \mathrm{~g} \cdot \mathrm{L}^{-1}$ lipase). Duodenum conditions $10 \mathrm{mM}$ phosphate buffer, $\mathrm{pH} 7.0, T=37^{\circ} \mathrm{C}$. The arrow indicates the moment that the lipolytic components are introduced. For clarity, only one replicate is shown for each tested condition, but similar plots were obtained with more replicates $(n \geq 2)$.

\section{Lipase}

As can be seen in Figure 4.4a (black line), lipase $\left(0.16 \mathrm{~g} \cdot \mathrm{L}^{-1}\right)$ reduced the interfacial tension at the bare oil-water interface more slowly than bile components at the concentrations used, which is comparable to results obtained by others (Torcello-Gómez, Maldonado-Valderrama, de Vicente, et al., 2011). From an interface perspective two mechanisms occur at the same time: lipase adsorption, which decreases the interfacial tension, followed by formation of surface-active lipolytic products (i.e., free fatty acids and monoacylglycerols) (Flipsen, van der Hijden, Egmond, \& Verheij, 1996; Reis, Holmberg, et al., 2008), that reduce the interfacial tension further, and in our case ultimately leading to detachment of the droplet from the capillary. Such an effect of the lipolysis reaction on interfacial tension was demonstrated in lipase/decane/tricaprylin systems, showing typically an interfacial tension of $7-8 \mathrm{mN} \cdot \mathrm{m}^{-1}$ for monoacylglycerols at the interface (Reis, Miller, et al., 2008).

When pre-adsorbed biopolymer films were used, introduction of lipase in the continuous phase led to unexpected behaviour (Figure 4.4b and c, black line): first, the interfacial tension increased at a similar rate for the single protein and four-layered film (24 \pm 8.6 $\left.\mu \mathrm{N} \cdot \mathrm{m}^{-1} \cdot \mathrm{s}^{-1}\right)$, reaching a value of about $17.9 \mathrm{mN} \cdot \mathrm{m}^{-1}$ after which the interfacial tension decreased $\left(-6.1 \pm 2.3 \mu \mathrm{N} \cdot \mathrm{m}^{-1} \cdot \mathrm{s}^{-1}\right)$ and levelled off again to $\gamma=7.8 \mathrm{mN} \cdot \mathrm{m}^{-1}$. To the best of our knowledge, such a non-monotonic change in interfacial tension has not been reported before 
(Maldonado-Valderrama et al., 2013; Marze, Meynier, \& Anton, 2013; Reis, Miller, et al., 2008), and although unfavourable thermodynamically, it could be explained if desorption of protein is faster than adsorption of lipase. This process may actually be induced by the lipase that wedges in between proteins, in which it may be aided by electrostatic repulsion between protein and lipase. These findings indicate that the replacement mechanism by lipase is clearly not driven by a reduction in interfacial tension only. After the lipase has anchored to the interface, lipolytic products are formed, resulting in lowering of the interfacial tension as by monoacylglycerols at the interface (Reis, Miller, et al., 2008).

After exposure to lipase, dilatational deformation was applied. The presence of lipase largely reduced the elasticity of the interfacial films, independent of deformation and pre-adsorbed biopolymers (average $E_{\mathrm{d}}$ of $5.0 \pm 0.3 \mathrm{mN} \cdot \mathrm{m}^{-1}$; Supplementary Figure 4.7). The maximum surface pressure response was clearly lowered in the presence of lipase (Table 4.2), both for single protein and four-layered film, and even lower than for bile, indicating a rapid exchange of molecules between bulk and interface. In first instance, the adsorbed layers are removed (see Figure 4.4), which affects surface elasticity largely. On top of that, lipolytic products formed in the oil phase may induce a low elastic response (Flipsen et al., 1996; Reis, Miller, et al., 2008); Berton-Carabin et al. found that surface-active lipid oxidation products generated in the oil phase largely decreased the elasticity of whey protein films at the oilwater interface (Berton-Carabin, Schröder, Rovalino Cordova, Schroën, \& Sagis, 2016).

\section{Mixed bile-lipase}

When used as a mixture, bile and lipase gave the same response on a bare oil-water interface as found for bile only (Figure 4.4a, brown line), indicating that bile components dominate the interfacial tension reduction, which can be expected for low molecular weight surfactants (i.e., bile salts) compared to proteins (Maldonado-Valderrama et al., 2015; Sagis \& Scholten, 2014). Bile components have previously been reported to affect lipase adsorption in various ways, ranging from promoting effects (Maldonado-Valderrama et al., 2013; TorcelloGómez, Maldonado-Valderrama, de Vicente, et al., 2011) to complete inhibition (Reis et al., 2009). Besides, lipolysis is an efficient self-regulation mechanism (Reis et al., 2009), for example monoacylglycerol molecules can expel lipase from the oil-water interface and thereby inhibit its action (Reis, Holmberg, et al., 2008).

When the oil-water interface was initially covered by biopolymers (single whey protein film or four-layered film), similar responses were found when comparing bile with bile/lipase mixture. The effects were slightly more extreme (drop detachment) than for bile only, suggesting accumulation of lipolysis products at the oil-water interface as observed by others for $\beta$-lactoglobulin films exposed to duodenal lipolytic conditions ( $1 \mathrm{mM}$ bile, $0.16 \mathrm{~g} \cdot \mathrm{L}^{-1}$ lipase) (Maldonado-Valderrama et al., 2013). For both films, the drop was lost before a 
plateau was reached, which implies that interfacial rheology measurements could not be conducted. In general, it can be concluded that since no clear differences were observed between the single protein- or four-layered interfacial film, desorption of the additional material took place rapidly and no protective effect of the biopolymer layers against lipolysis was observed at the concentrations used here. These findings are in line with in situ observations for emulsion systems under simulated intestinal conditions, showing that addition of a biopolymer layer onto emulsion droplets did not change the initial rate of displacement by bile and lipase (Pan \& Nitin, 2016).

\subsubsection{Adsorption-desorption kinetics}

The formation, behaviour and possible destabilization of interfacial films in simulated duodenal conditions were also studied by reflectometry, which gives information about the adsorbed amounts and adsorption rates.

First, a protein film was constructed at $\mathrm{pH} 3.0$ on a hydrophobic surface, and non-adsorbed or loosely attached material was removed by a buffer-rinse. Subsequent exposure to duodenal $\mathrm{pH}$ conditions (7.0) induced a slight desorption of about $0.2 \mathrm{mg} \cdot \mathrm{m}^{-2}$, but $0.8 \mathrm{mg} \cdot \mathrm{m}^{-2}$ was irreversibly adsorbed (start of Figure 4.5). Once a plateau was reached, the obtained film was exposed to bile, lipase, or a combination thereof.

Bile extract adsorbed rapidly to the biopolymer film, and within $5 \mathrm{~min}$, a stable amount of adsorbed material was reached $\left(1.63 \pm 0.07 \mathrm{mg} \cdot \mathrm{m}^{-2}\right)$. This confirms that bile has a high affinity for the surface, allowing fast adsorption onto a pre-adsorbed biopolymer film. During subsequent buffer rinse, the desorption rate of bile was high as well; this reversibility suggests that interactions with the surface are weak, as are interactions between bile molecules (Maldonado-Valderrama et al., 2011). This is in agreement with dilatational rheology measurements that showed low maximum surface pressures in compression/expansion (Table 4.2).

Lipase also showed a high initial adsorption rate, similar to that of bile, which levelled off after 5 min but did not reach a plateau within $30 \mathrm{~min}$, although it should be mentioned that the maximum adsorbed amount $\left(2.74 \pm 0.11 \mathrm{mg} \cdot \mathrm{m}^{-2}\right)$ was more than three-times as high as that of the initial protein material, and higher than that of bile. The desorption rate of lipase was much lower than that of bile, indicative of its less reversible adsorption, and seemed to show two stages: an initial drop due to removal of loosely attached material, followed by slower desorption. These results suggest that lipase molecules adsorb strongly to the hydrophobic surface, and the effect is only partially reversible, as was found for the hydrophobic surface itself (Schroen et al., 1995). In the droplet volume tensiometer possibly 
lipolysis products exhibited an additional effect on lipase, leading to removal from the interface (Maldonado-Valderrama et al., 2013).

For simultaneous exposure to bile and lipase, the same effects were noted as for bile only, but the adsorbed amounts were higher $\left(2.09 \pm 0.29 \mathrm{mg} \cdot \mathrm{m}^{-2}\right)$. This confirms that bile adsorption took place first, followed by co-adsorption of lipase, albeit in strong competition with bile. The desorption profile looked like a combination of the individual components, with an initial drop as for bile and a slower second phase as for lipase, indicative of a mixed interface.

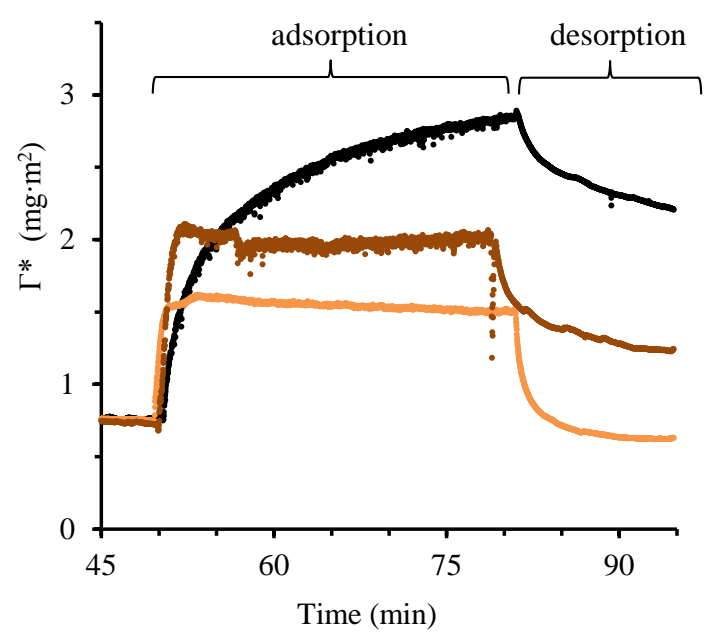

Figure 4.5. Evolution of adsorbed amount $\left(\Gamma^{*}\right)$ during adsorption and desorption of bile (orange), lipase (black) or a combination (brown) at a pre-adsorbed protein layer exposed to $\mathrm{pH} 7.0$ prior to adsorption (i.e., introduction of the lipolytic components) and sequentially (i.e., desorption), investigated by reflectometry. For clarity, only one replicate is shown for each tested condition, but similar plots were obtained with more replicates $(n=3)$.

The use of both methods has allowed us to investigate effects occurring at complex interfaces in great detail. Besides the methods allow for distinction between adsorption-related effects, and effects caused by the formation of reaction products (i.e., free fatty acids and monoacylglycerols). In this way, mechanistic understanding is generated for the situation in the duodenum, but the methods are very flexible and will also allow investigation of rather different conditions that are present elsewhere in the GI tract. Besides, we think that the combination of methods is a valuable starting point for encapsulate design, for which the effects of different physiological conditions can be tested fast, and immediately related to the digestive fate of the formulation. 


\subsection{CONCLUSIONS}

Our results show that the elastic behaviour of multilayered protein/pectin films is driven by the first protein layer that is in direct contact with the oil phase, in which multiple and strong intermolecular interactions are established. Additional layers adsorbed through electrostatic interactions hardly add to the interfacial tension decrease and film elasticity. At $\mathrm{pH} 7.0$, adsorbed material was removed from the interface, leading to lower elasticity, comparable to that found for a single protein layer.

When exposed to bile, lipase, or a combination thereof, it was found that bile components partly replaced pre-adsorbed biopolymers. A viscoelastic film was formed with low mechanical strength, indicating highly dynamic adsorption/desorption. Lipase was also able to remove material from the oil-water interface, albeit through a different mechanism. First the protein was removed, after which reaction products were formed, that possibly in turn removed lipase from the interface. When bile and lipase are both present, bile molecules rule the initial response, with lipase co-adsorbing and generating reaction products.

The unique combination of methods that we used here provides detailed information on emulsion droplet digestion under duodenal conditions. We believe such information will be valuable in further development of $\mathrm{O} / \mathrm{W}$ emulsion systems used as delivery systems for functional ingredients.

\section{Acknowledgement}

Research presented in this publication was financially supported by the Graduate School VLAG. 


\subsection{SUPPLEMENTARY INFORMATION}

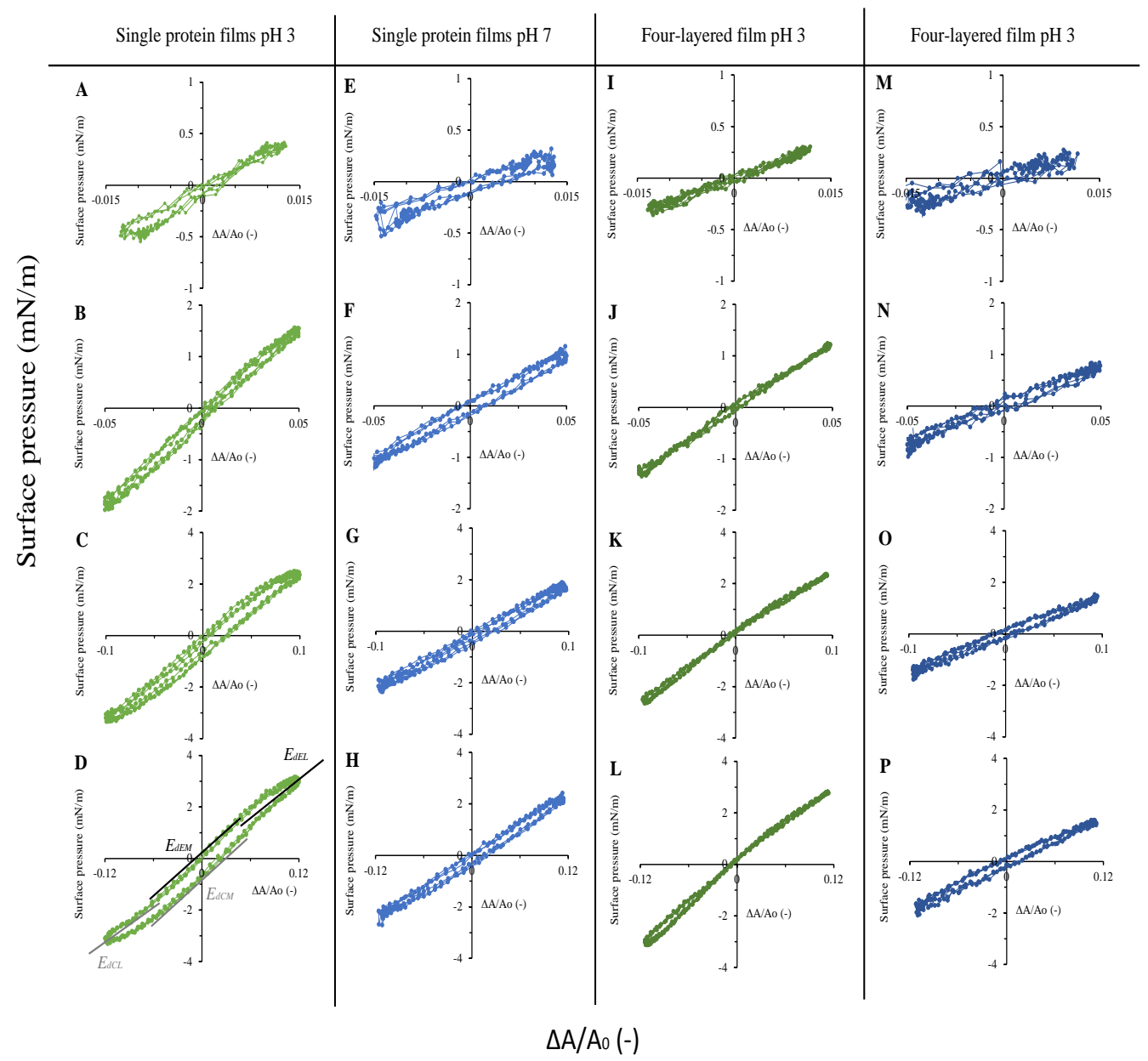

Supplementary Figure 4.6. Lissajous plot of surface pressure versus deformation after exposing a single protein film to lipase (A-C) or bile (D-F), and a four-layered film to lipase (G-I). Deformation up to $1 \%$ (A, E, I, M), 5\% (B, F, J, N), 10\% (C, G, K, O) and 12\% (D, H, L, P). 

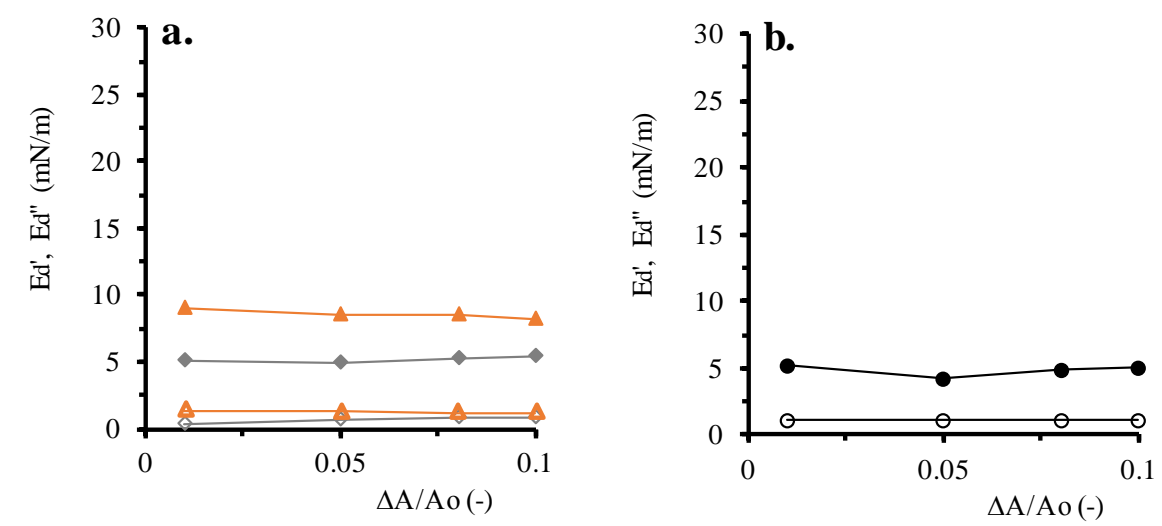

Supplementary Figure 4.7. Dilatational elastic ( $E$ ' closed symbols) and viscous ( $E$ ” open symbols) moduli as a function of the applied deformation. Comparing interfacial behaviour of a) single protein film exposed to lipase (grey, diamonds) or bile (orange, triangles) and b) four-layered film exposed to lipase (black, circles), all at $\mathrm{pH} 7.0, T=37^{\circ} \mathrm{C}$.

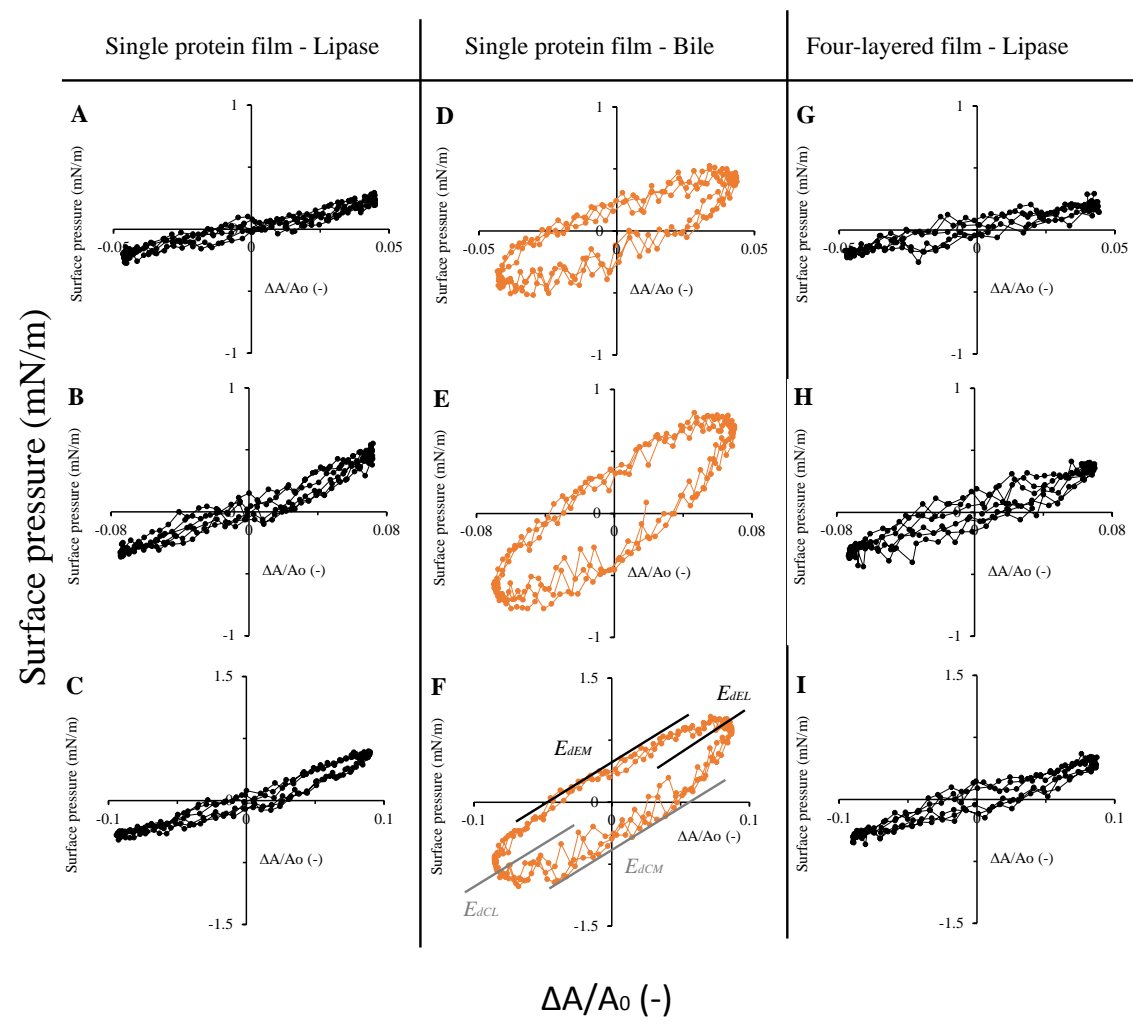

Supplementary Figure 4.8. Lissajous plot of surface pressure versus deformation after exposing a single protein film to lipase (A-C) or bile (D-F), and a four-layered film to lipase (G-I). Deformation up to $5 \%$ (A, D, G), 8\% (B, E, H), 10\% (C, F, I). 


\subsection{REFERENCES}

Bakala N’Goma, J.-C., Amara, S., Dridi, K., Jannin, V., \& Carrière, F. (2012). Understanding the lipiddigestion processes in the GI tract before designing lipid-based drug-delivery systems. Therapeutic Delivery, 3(1), 105-124.

Bellesi, F. A., Pizones Ruiz-Henestrosa, V. M., \& Pilosof, A. M. R. (2014). Behavior of protein interfacial films upon bile salts addition. Food Hydrocolloids, 36, 115-122.

Benjamins, J., Cagna, A., \& Lucassen-Reynders, E. H. (1996). Viscoelastic properties of triacylglycerol/water interfaces covered by proteins. Colloids and Surfaces A: Physicochemical and Engineering Aspects, 114, 245-254.

Benjamins, J., Lyklema, J., \& Lucassen-Reynders, E. H. (2006). Compression/expansion rheology of oil/water interfaces with adsorbed proteins. Comparison with the air/water surface. Langmuir, 22(14), 6181-6188.

Berton-Carabin, C. C., Schröder, A., Rovalino Cordova, A., Schroën, K., \& Sagis, L. M. C. (2016). Protein and lipid oxidation affect the viscoelasticity of whey protein layers at the oil-water interface. European Journal of Lipid Science and Technology, 118, 1630-1643.

Bos, M. A., \& Vliet, T. Van. (2001). Interfacial rheological properties of adsorbed protein layers and surfactants : a review. Advances in Colloid and Interface Science, 91, 437-471.

Chu, B.-S., Gunning, A. P., Rich, G. T., Ridout, M. J., Faulks, R. M., Wickham, M. S. J., ... Wilde, P. J. (2010). Adsorption of bile salts and pancreatic colipase and lipase onto digalactosyldiacylglycerol and dipalmitoylphosphatidylcholine monolayers. Langmuir, 26(12), 9782-93.

Chu, B.-S., Rich, G. T., Ridout, M. J., Faulks, R. M., Wickham, M. S. J., \& Wilde, P. J. (2009). Modulating pancreatic lipase activity with galactolipids: effects of emulsion interfacial composition. Langmuir, 25(16), 9352-9360.

Corstens, M. N., Berton-Carabin, C. C., de Vries, R., Troost, F. J., Masclee, A. A. M., \& Schroën, K. (2017a). Food-grade Micro-encapsulation Systems that May Induce Satiety via Delayed Lipolysis: A Review. Critical Reviews in Food Science and Nutrition, 57(10):2218-2244.

Corstens, M. N., Berton-Carabin, C. C., Kester, A., Fokkink, R., van den Broek, J. M., de Vries, R., ... Schroën, K. (2017b). Destabilization of multilayered interfaces in digestive conditions limits their ability to prevent lipolysis in emulsions. Food Structure, 12:54-63.

del Castillo-Santaella, T., Maldonado-Valderrama, K., Cabrerizo-Vílchez, M. A., Rivadeneira-Ruiz, C., Rondón-Rodriguez, D., \& Gálvez-Ruiz, M. J. (2015). Natural Inhibitors of Lipase: Examining Lipolysis in a Single Droplet. Journal of Agricultural and Food Chemistry, 63(47), 10333-10340.

Deshpande, A. P., Murali Krishnan, J., \& Sunil Kumar, P. B. (2010). Rheology of Complex Fluids. Springer Science+Business Media.

Dijt, J. C., Stuart, M. A. C., \& Fleer, G. J. (1994). Reflectometry as a tool for adsorption studies. Advances in Colloid and Interface Science, 50, 79-101.

Euston, S. R., Bellstedt, U., Schillbach, K., \& Hughes, P. S. (2011). The adsorption and competitive adsorption of bile salts and whey protein at the oil-water interface. Soft Matter, 7(19), 8942.

Flipsen, J. A. C., van der Hijden, H. T. W. M., Egmond, M. R., \& Verheij, H. M. (1996). Action of cutinase at the triolein-water interface. Characterisation of interfacial effects during lipid hydrolysis using the oil-drop tensiometer as a tool to study lipase kinetics. Chemistry and Physics of Lipids, 84(2), 105115.

Ganzevles, R. A., Fokkink, R., van Vliet, T., Stuart, M. A. C., \& de Jongh, H. H. J. (2008). Structure of mixed b-lactoglobulin/pectin adsorbed layers at air/water interfaces; a spectroscopy study. Journal of Colloid and Interface Science, 317(1), 137-147.

Ganzevles, R. A., Kosters, H., van Vliet, T., Stuart, M. A. C., \& de Jongh, H. H. J. (2007). Polysaccharide charge density regulating protein adsorption to air/water interfaces by protein/polysaccharide complex formation. Journal of Physical Chemistry B, 111(45), 12969-12976.

Ganzevles, R. A., Zinoviadou, K., van Vliet, T., Stuart, M. A. C., \& de Jongh, H. H. J. (2006). Modulating Surface Rheology by Electrostatic Protein / Polysaccharide Interactions Modulating Surface Rheology by Electrostatic Protein / Polysaccharide Interactions. Society, 108(16), 10089-10096.

Hu, M., Li, Y., Decker, E. A., Xiao, H., \& McClements, D. J. (2011). Impact of Layer Structure on Physical Stability and Lipase Digestibility of Lipid Droplets Coated by Biopolymer Nanolaminated Coatings. Food Biophysics, 6, 37-48. 
Jourdain, L. S., Schmitt, C., Leser, M. E., Murray, B. S., \& Dickinson, E. (2009). Mixed layers of sodium caseinate + dextran sulfate: Influence of order of addition to oil-water interface. Langmuir, 25(16), 10026-10037.

Klinkesorn, U., \& McClements, D. J. (2010). Impact of lipase, bile salts, and polysaccharides on properties and digestibility of tuna oil multilayer emulsions stabilized by lecithin-chitosan. Food Biophysics, 5(2), 73-81.

Lesmes, U., Baudot, P., \& McClements, D. J. (2010). Impact of interfacial composition on physical stability and in vitro lipase digestibility of triacylglycerol oil droplets coated with lactoferrin and/or caseinate. Journal of Agricultural and Food Chemistry, 58(13), 7962-7969.

Li, Y., Hu, M., Xiao, H., Du, Y., Decker, E. A., \& McClements, D. J. (2010). Controlling the functional performance of emulsion-based delivery systems using multi-component biopolymer coatings. European Journal of Pharmaceutics and Biopharmaceutics, 76(1), 38-47.

Li, Y., \& McClements, D. J. (2014). Influence of cosurfactant on the behavior of structured emulsions under simulated intestinal lipolysis conditions. Food Hydrocolloids, 40, 96-103.

Lucassen-Reynders, E. H., Benjamins, J., \& Fainerman, V. B. (2010). Dilational rheology of protein films adsorbed at fluid interfaces. Current Opinion in Colloid and Interface Science, 15(4), 264-270.

Mackie, A., Gunning, A., Wilde, P., \& Morris, V. (1999). Orogenic Displacement of Protein from the Air/Water Interface by Competitive Adsorption. Journal of Colloid and Interface Science, 210(1), 157-166.

Maldonado-Valderrama, J., Gálvez-Ruiz, M. J., Martín-Rodríguez, A., \& Cabrerizo-Vílchez, M. A. (2004). Adsorbed and spread $\beta$-casein monolayers at oil-water interfaces. Langmuir, 20(15), 6093-6095.

Maldonado-Valderrama, J., Holgado-Terriza, J. A., Torcello-Gómez, A., \& Cabrerizo-Vílchez, M. A. (2013). In vitro digestion of interfacial protein structures. Soft Matter, 9(4), 1043-1053.

Maldonado-Valderrama, J., Miller, R., Fainerman, V. B., Wilde, P. J., \& Morris, V. J. (2010). Effect of gastric conditions on b-lactoglobulin interfacial networks: Influence of the oil phase on protein structure. Langmuir, 26(20), 15901-15908.

Maldonado-Valderrama, J., Torcello-Gómez, A., del Castillo-Santaella, T., Holgado-Terriza, J. A., \& Cabrerizo-Vílchez, M. A. (2015). Subphase exchange experiments with the pendant drop technique. Advances in Colloid and Interface Science, 222, 488-501.

Maldonado-Valderrama, J., Wilde, P., Macierzanka, A., \& Mackie, A. (2011). The role of bile salts in digestion. Advances in Colloid and Interface Science, 165, 36-46.

Maldonado-Valderrama, J., Woodward, N. C., Patrick Gunning, A., Ridout, M. J., Husband, F. A., Mackie, A. R., ... Wilde, P. J. (2008). Interfacial characterization of B-lactoglobulin networks: Displacement by bile salts. Langmuir, 24(13), 6759-6767.

Marze, S., Meynier, A., \& Anton, M. (2013). In vitro digestion of fish oils rich in n-3 polyunsaturated fatty acids studied in emulsion and at the oil-water interface. Food \& Function, 4(2), 231-9.

McClements, D. J. (2005). Food Emulsions. Principles, Practices, and Techniques (Second Edi). United States of America.

Miller, R., Grigoriev, D. O., Krägel, J., Makievski, A. V, Maldonado-Valderrama, J., Leser, M., ... Fainerman, V. B. (2005). Experimental studies on the desorption of adsorbed proteins from liquid interfaces. Food Hydrocolloids, 19(3), 479-483.

Minekus, M., Alminger, M., Alvito, P., Ballance, S., Bohn, T., Bourlieu, C., ... Brodkorb, A. (2014). A standardised static in vitro digestion method suitable for food - an international consensus. Food \& Function, 5, 1113-24.

Mun, S., Decker, E. A., Park, Y., Weiss, J., \& McClements, D. J. (2006). Influence of Interfacial Composition on in vitro Digestibility of Emulsified Lipids: Potential Mechanism for Chitosan's Ability to Inhibit Fat Digestion. Food Biophysics, 1(1), 21-29.

Pan, Y., \& Nitin, N. (2016). Real-time measurements to characterize dynamics of emulsion interface during simulated intestinal digestion. Colloids and Surfaces B: Biointerfaces, 141, 233-241.

Reis, P., Holmberg, K., Miller, R., Krägel, J., Grigoriev, D. O., Leser, M. E., \& Watzke, H. J. (2008). Competition between lipases and monoglycerides at interfaces. Langmuir, 24(14), 7400-7.

Reis, P., Holmberg, K., Watzke, H., Leser, M. E., \& Miller, R. (2009). Lipases at interfaces: A review. Advances in Colloid and Interface Science, 147-148, 237-250.

Reis, P., Miller, R., Krägel, J., Leser, M., Fainerman, V., Watzke, H., \& Holmberg, K. (2008). Lipases at interfaces: Unique interfacial properties as globular proteins. Langmuir, 24(13), 6812-6819.

Rossier-Miranda, F. J., Schroën, K., \& Boom, R. (2010). Mechanical characterization and pH response of fibril-reinforced microcapsules prepared by layer-by-layer adsorption. Langmuir, 26(24), 1910619113. 
Rühs, P., Scheuble, N., Windhab, E., \& Fischer, P. (2013). Protein adsorption and interfacial rheology interfering in dilatational experiment. European Physical Journal: Special Topics, 222(1), 47-60.

Sagis, L. M. C., \& Fischer, P. (2014). Nonlinear rheology of complex fluid-fluid interfaces. Current Opinion in Colloid and Interface Science, 19(6), 520-529.

Sagis, L. M. C., \& Scholten, E. (2014). Complex interfaces in food: Structure and mechanical properties. Trends in Food Science \& Technology, 37(1), 59-71.

Sarkar, A., Murray, B., Holmes, M., Ettelaie, R., Abdalla, A., \& Yang, X. (2016). In vitro digestion of Pickering emulsions stabilized by soft whey protein microgel particles: influence of thermal treatment. Soft Matter, 12(15), 3558-69.

Sarkar, A., Ye, A., \& Singh, H. (2016). On the role of bile salts in the digestion of emulsified lipids. Food Hydrocolloids, 60, 77-84.

Schmidt, U. S., Schütz, L., \& Schuchmann, H. P. (2016). Interfacial and emulsifying properties of citrus pectin: Interaction of $\mathrm{pH}$, ionic strength and degree of esterification. Food Hydrocolloids.

Schönhoff, M. (2003). Layered polyelectrolyte complexes : physics of formation and molecular properties. Journal of Physics: Condensed Matter, 15, 1781-1808.

Schröen, C. G. P. H., Cohen Stuart, M. A., Van der Padt, A., \& Van’t Riet, K. (1994). Wettability of tri-block copolymer coated hydrophobic surfaces Predictions and measurements. Colloids and Surfaces A: Physicochemical and Engineering Aspects, 90(2-3), 235-249.

Schroen, C. G. P. H., Stuart, M. A. C., Maarschalk, K. V., Vanderpadt, A., \& Vantriet, K. (1995). Influence of Preadsorbed Block-Copolymers on Protein Adsorption: Surface Properties, Layer Thickness, and Surface Coverage. Langmuir, 11(8), 3068-3074.

Tokle, T., Mao, Y., \& McClements, D. J. (2013). Potential biological fate of emulsion-based delivery systems: lipid particles nanolaminated with lactoferrin and $\beta$-lactoglobulin coatings. Pharmaceutical Research, 30(12), 3200-3213.

Torcello-Gómez, A., Maldonado-Valderrama, J., de Vicente, J., Cabrerizo-Vílchez, M. A., Gálvez-Ruiz, M. J., \& Martín-Rodríguez, A. (2011). Investigating the effect of surfactants on lipase interfacial behaviour in the presence of bile salts. Food Hydrocolloids, 25(4), 809-816.

Torcello-Gómez, A., Maldonado-Valderrama, J., Jódar-Reyes, A. B., \& Foster, T. J. (2013). Interactions between Pluronics (F127 and F68) and Bile Salts (NaTDC) in the Aqueous Phase and the Interface of Oil-in-Water Emulsions. Langmuir, 29, 2520-2529.

Torcello-Gómez, A., Maldonado-Valderrama, J., Martín-Rodríguez, A., \& McClements, D. J. (2011). Physicochemical properties and digestibility of emulsified lipids in simulated intestinal fluids: influence of interfacial characteristics. Soft Matter, 7(13), 6167.

Tzoumaki, M. V, Moschakis, T., Scholten, E., \& Biliaderis, C. G. (2013). In vitro lipid digestion of chitin nanocrystal stabilized o/w emulsions. Food \& Function, 4(1), 121-9.

Verhoeckx, K., Cotter, P., Kleiveland, C., Lea, T., Mackie, A., \& Requena, T. (2015). The Impact of Food Bioactives on Health. Springer Open.

Wilde, P. J., \& Chu, B. S. (2011). Interfacial \& colloidal aspects of lipid digestion. Advances in Colloid and Interface Science, 165(1), 14-22.

Wulff-Pérez, M., Vicente, J. De, Martín-rodríguez, A., \& Gálvez-Ruiz, M. J. (2012). Controlling lipolysis through steric surfactants : New insights on the controlled degradation of submicron emulsions after oral and intravenous administration. International Journal of Pharmaceutics, 423(2), 161-166.

Yao, X., Zhang, W., Nie, K., Gao, Z., Fang, Y., Nishinari, K., ... Jiang, F. (2016). Effect of Gum Arabic, Gum Ghatti and Sugar Beet Pectin as Interfacial Layer on Lipid Digestibility in Oil-in-Water Emulsions. Food Biophysics, 11(3), 292-301. 


\title{
Chapter 5
}

\section{Emulsion-alginate beads:}

\author{
static in vitro digestion
}

This chapter has been published as Corstens M.N., Berton-Carabin C.C., Elichiry-Ortiz P.T., Hol K., Troost F.J., Masclee A.A.M., Schroën K. (2017) Emulsion-alginate beads designed to control in vitro intestinal lipolysis: Towards appetite-control. Journal of Functional Foods 34:319-28. 


\section{ABSTRACT}

Dietary lipids and digestion products are strong inducers of satiety signals in the distal small intestine. To protect lipids against proximal absorption we encapsulate them in hydrogel beads. Physically stable beads of different sizes (0.55, 0.78 and $1.15 \mathrm{~mm})$, and mesh sizes ( $\xi=9.2,6.4$ and $5.4 \mathrm{~nm}$ ) were obtained using ionotropic $(\mathrm{Ca})$ gelation of alginate containing oil-in-water $(\mathrm{O} / \mathrm{W})$ emulsions $\left(d_{32} \sim 21 \mu \mathrm{m}\right)$. All beads shrunk at $\mathrm{pH} 2.0$, and had excellent gastric stability (2 h, pepsin, $\mathrm{pH} 3.0$ ), while they swelled at $\mathrm{pH} 7.0$, and softened under simulated intestinal conditions (2.5 h, pancreatin, bile, pH 7.0). Lipolysis could be controlled through variation of bead and mesh size, resulting in a broad range of release profiles: from $1-50 \%$ release after $1 \mathrm{~h}$ to $20-80 \%$ after $2.5 \mathrm{~h}$. Such systems with controllable and predictable in vitro release profiles are a promising step towards ileal lipid release, where they could play a pivotal role in appetite control. 


\subsection{INTRODUCTION}

Exposing the small intestine to macronutrients can induce negative feedback mechanisms to the proximal gastrointestinal (GI) tract including stomach, gallbladder and pancreas but also to the central nervous system (Alleleyn, van Avesaat, Troost, \& Masclee, 2016; van Avesaat, Troost, Ripken, Hendriks, \& Masclee, 2015). This negative feedback process is able to inhibit food digestion, appetite sensations and food intake, and thus increases feelings of satiety and satiation (Alleleyn et al., 2016; Maljaars et al., 2011). This so-called ileal brake activation has been demonstrated for all macronutrients (Alleleyn et al., 2016), but was first described for lipids (Welch, Saunders, \& Read, 1985; Welch, Sepple, \& Read, 1988). Lipids have proven to act as potent ileal brake activators when directly infused to the targeted area (via a naso-ileal catheter). This concept is relevant to include in long-term weight management strategies. However, for successful weight management in a free-living setting, ileal brakeinducing lipids should preferably be ingested via the normal oral route of food intake instead of by tube-fed ileal delivery. Under physiological GI conditions, the major part of dietary lipids is digested and absorbed in the proximal small intestine (Bakala N'Goma, Amara, Dridi, Jannin, \& Carrière, 2012), and hence does not reach the ileum. Thus, in order to induce ileal brake activation, dietary lipids need to be protected against the action of digestive components.

Since lipolysis occurs at the surface of lipid droplets (i.e., the oil-water interface), the total interfacial area and accessibility of this interface are key in controlling lipolysis (Golding et al., 2011; Lundin, Golding, \& Wooster, 2008), and many attempts have been made to control lipid digestion through the design of a protective interface around lipid droplets. Some interfacial components were claimed to delay intestinal lipolysis to a certain extent, such as surfactants through steric hindrance (Chu et al., 2009; Wulff-Pérez, Vicente, MartínRodríguez, \& Gálvez-Ruiz, 2012), biopolymers through thicker interfacial films produced by layer-by-layer adsorption (Corstens et al., 2017b; Hu, Li, Decker, Xiao, \& McClements, 2011; Klinkesorn \& McClements, 2010; Mun, Decker, Park, Weiss, \& McClements, 2006; Zeeb, Lopez-Pena, Weiss, \& McClements, 2015), and Pickering particles possibly through irreversible adsorption (Sarkar et al., 2016; Tzoumaki, Moschakis, Scholten, \& Biliaderis, 2013). In general, the protective effect was not very pronounced, especially in cases where protection against both the gastric and intestinal phases was targeted (Corstens et al., 2017a).

Alternatively, the accessibility of the oil-water interface can be reduced when the oil droplets are embedded in a matrix that controls the diffusion of the lipolytic components (i.e., lipase, bile salts) and of lipolytic products. For instance, hydrogels have been shown to control in vitro lipolysis, both when the gels themselves are digestible (Dekkers, Kolodziejczyk, Acquistapace, Engmann, \& Wooster, 2016; Sarkar et al., 2015) or not (Li, Hu, Du, Xiao, \& McClements, 2011; Zhang, Zhang, Zou, Chen, et al., 2016). For digestible emulsion-filled 
protein gels (e.g., gelatin), surface erosion of the gel matrix itself by proteases was the major gel degradation mechanism (Sarkar et al., 2015). When the same gel was studied in the absence of proteases, the rate of in vitro lipolysis correlated with the average mesh size in the gel and with the diffusion distance, although a predictive model was not formulated. Lipase was able to diffuse through a gel when the average mesh size was 3-4 times the size of lipase, and the lipolysis rate was $\sim 130$ times slower compared to that in the free emulsion (Sarkar et al., 2015).

Hence, a non-digestible gel matrix is preferred to achieve better control over lipase diffusion, and good candidates are, for example, dietary fibres such as chitosan or alginate (Brownlee et al., 2005). Alginate can be cross-linked with divalent cations (as $\mathrm{Ca}^{2+}, \mathrm{Cu}^{2+}, \mathrm{Ba}^{2+}$ ) that act as cationic bridges mainly between sequences that are rich in guluronic acid residues (G-G sequences) along two aligned chains, forming an 'egg-box' structure (Bajpai \& Sharma, 2004; Grant, Morris, Rees, Smith, \& Thom, 1973; Stewart, Gray, Vasiljevic, \& Orbell, 2014). Encapsulation of emulsions in alginate beads has been described for controlled release of volatiles in the mouth (Lian, Malone, Homan, \& Norton, 2004; Malone \& Appelqvist, 2003), controlled in vivo drug delivery in the stomach (floating beads) (Murata, Sasaki, Miyamoto, \& Kawashima, 2000; Whitehead, Fell, Collett, Sharma, \& Smith, 1998), and gastric protection for prolonged intestinal drug release (Arica et al., 2005).

This efficient and controlled release behaviour is a result of the physical characteristics of alginate beads, such as their size (Li, Hu, Du, et al., 2011; Rayment et al., 2009; Zeeb, Saberi, Weiss, \& McClements, 2015), and mesh size (Wright et al., 2009) as function of the environmental conditions. Under gastric conditions, calcium-alginate beads were reported to shrink, under intestinal conditions to swell, and ultimately to disintegrate at the end of the intestinal phase (Rayment et al., 2009). Besides, it was reported that the gel structure is not necessarily homogeneous across the bead: under intestinal conditions, alginate beads were shown to have larger pores in the core, while a denser structure remained at the edge (Wright et al., 2009). These observations show that alginate beads physically respond to changes in environmental conditions as encountered in the GI tract, and constitute therefore an attractive option for the controlled release of dietary lipids.

A preliminary study on fatty acid release from nanoemulsion-filled alginate beads investigated the effect of bead size, lipid type, and calcium and alginate concentration, in an in vitro model of the small intestine (Li, Hu, Du, et al., 2011). In other studies where a gastric phase was included prior to in vitro intestinal lipolysis, lipolysis was also reported to be delayed compared to conventional emulsions (Zhang, Zhang, Zou, Chen, et al., 2016; Zhang, Zhang, \& McClements, 2016; Zhang, Zhang, Zou, \& McClements, 2016). However, in all these studies the bead design was not systematically linked to the observed behaviour in both 
gastric and intestinal phases, and this is, in our opinion, what is needed to bring such systems to the next level of application.

The purpose of the present study was to encapsulate micron-sized emulsion droplets in alginate beads with different matrix mesh sizes and diffusion distances (i.e., bead size). We evaluated physical properties of the beads in simulated GI conditions $(\mathrm{pH}$, ions, presence of enzymes), and measured GI lipolysis under conditions representing the postprandial state. A mathematical model was used to link lipolysis kinetics to bead design, which helped us develop guidelines for the design of alginate beads for controlled intestinal release of dietary lipids.

\subsection{MATERIAL AND METHODS}

\subsubsection{Materials}

Safflower oil was purchased from De Wit Specialty oils (19200 Safflower Oil High Linoleic Refined, The Netherlands). Whey protein isolate (WPI) was obtained from Davisco Foods International (BiPro, Eden Prairie, Minnesota, USA; purity 97.5\%). Sodium alginate (W201502, medium viscosity (5-40 cps)), sodium phosphate monobasic, sodium phosphate dibasic, sodium hydroxide, hydrochloric acid, sodium chloride, calcium chloride, hexane, isopropanol, pepsin from porcine gastric mucosa (P7125), pancreatin from porcine pancreas (P7545, $8 \times$ USP specification; including trypsin, amylase, lipase, ribonuclease, protease), and porcine bile extract (B8631, crude extract, purity estimated to be 30-60\%) were purchased from Sigma Aldrich (St. Louis, MO, USA). Enzyme activities were measured according to Minekus et al. (2014), and were $41 \mathrm{U} \cdot \mathrm{mg}^{-1}$ for pancreatin, and $>400 \mathrm{U} \cdot \mathrm{mg}^{-1}$ for pepsin. All materials were used directly without further purification. Ultrapure water, purified by a Millipore Milli-Q system (Darmstadt, Germany), was used throughout the study.

\subsubsection{Methods}

\section{$\underline{\text { Preparation of emulsion-filled alginate beads }}$}

Emulsion preparation. WPI solution $\left(10 \mathrm{~g} \cdot \mathrm{L}^{-1}\right)$ was prepared in $10 \mathrm{mM}$ phosphate buffer $\mathrm{pH}$ 7.0 and gently stirred for at least $4 \mathrm{~h}$ at room temperature. Safflower oil (20 wt\%) was added to the WPI solution and both phases were mixed using a rotor stator homogeniser (Ika T18 basic Ultra-Turrax homogenizer equipped with a S18N-19G dispersion tool, Staufen, Germany) twice for $1 \mathrm{~min}$ at 11,000 rpm, with a 30 second break in between, to obtain an emulsion $\left(d_{32}=21 \pm 4 \mu \mathrm{m}\right)$.

Emulsion-filled alginate bead preparation. Alginate solutions (in concentrations 18, 36, 54 $\mathrm{g} \cdot \mathrm{L}^{-1}$ ) were prepared in $10 \mathrm{mM}$ phosphate buffer $\mathrm{pH} 7.0$ and gently stirred for at least $4 \mathrm{~h}$ at 
room temperature. Freshly prepared emulsion (within 5 min of production) was added to the alginate solution in volume ratio 1:1 (final composition: $10 \mathrm{wt} \%$ oil, with $10,20,30 \mathrm{~g} \cdot \mathrm{L}^{-1}$ alginate in the aqueous phase) and mixed at a rate of $400 \mathrm{rpm}$ for $30 \mathrm{~min}$ and left for $30 \mathrm{~min}$. After that, the alginate-emulsion mixture was added dropwise into a gently stirring hardening bath ( $5 \mathrm{wt} \% \mathrm{CaCl}_{2}$ in ultrapure water) with a syringe pump (flow rate $1.0 \mathrm{~mL} \cdot \mathrm{min}^{-1}$ ). The nozzle tip (inner diameter $0.41 \mathrm{~mm}$; Nordson EFD, U.K.) was positioned 5-6 cm above the bath. The bead size could be controlled by only the air flow along the nozzle that generated a drag force (Workamp, Alaie, \& Dijksman, 2016), leading to beads between 0.3-1.7 mm (irrespective of the liquid flow rate and alginate concentration; more details in Supplementary Figure 5.8b). After production, the beads were stirred in the calcium bath at room temperature for at least 30 more min, and left overnight at $4{ }^{\circ} \mathrm{C}$ to harden. The size of the beads was monitored over time $\left(50 \mathrm{~d}, 4{ }^{\circ} \mathrm{C}, 5 \mathrm{wt} \% \mathrm{CaCl}_{2}\right)$.

Before use, the beads were taken from the $\mathrm{CaCl}_{2}$ solution and washed with ultrapure water ( $20^{\circ} \mathrm{C}$ ), using fast ashless filter paper (grade 41, $20 \mu \mathrm{m}$ pores, No. 1441-090, Whatman International, U.K.).

For reference measurements, calcium alginate beads without emulsion were produced from $20 \mathrm{~g} \cdot \mathrm{L}^{-1}$ alginate solution in $10 \mathrm{mM}$ phosphate $\mathrm{pH}$ 7.0, as described above, and exposed to in vitro digestion conditions.

\section{Physical characterisation}

Emulsion droplet size distribution. The droplet size distribution of the emulsions was determined using static light scattering (Mastersizer 2000 with Hydro SM dispersion unit, Malvern Instruments, UK). The refractive index of safflower oil was set at 1.460 and that of the dispersant at 1.333 .

Bead size. Beads were imaged against a black background at a resolution of 600 dpi. From the obtained images, the area of at least 50 beads (circularity $0.7-1.0$ ) was determined with ImageJ software (version 1.51f, NIH, Maryland, USA), from which the diameter was derived.

Oil content. The oil content in the beads was determined as described before (Corstens et al., 2017b). In short, a known amount of beads ( $\pm 1 \mathrm{~g}$ ) was added to a known amount of hexane/2propanol (3:2 (v/v) ratio) mixture $( \pm 15-20 \mathrm{~g})$ and processed using a rotor stator homogeniser (Ika T18 basic Ultra-Turrax homogenizer equipped with a S18N-19G dispersion tool, Staufen, Germany) to disrupt the beads ( $1 \mathrm{~min}$ at 10,000 rpm in combination with 2 min at 15,000 rpm). The mixture was centrifuged (5 min at 3,000 rpm, $20^{\circ} \mathrm{C}$, ThermoScientific, Legend XFR) to speed up separation into two phases. A known amount of the hexane phase was dried, and the amount of oil in the initial emulsion was calculated. 
Light microscopy. The visual appearance of the beads was analysed using light microscopy (Zeiss Axioscope A1, Axiocam Mrc 5), at 10-time magnification. Multiple images were made to obtain an overview of the sample; the method is limited to beads below $1 \mathrm{~mm}$.

$X$-ray tomography. For 3D non-invasive and non-destructive imaging a GE Phoenix v|tome|x m tomographer (General Electric, Wunstorf, Germany) was used. The system contains two $\mathrm{X}$-ray sources. The $180 \mathrm{kV}$ nano-focus tube with tungsten target was employed. X-rays were produced with a voltage of $80 \mathrm{kV}$ and a current of $180 \mu \mathrm{A}$. The images were recorded by a GE DXR detector array with $2024 \times 2024$ pixels (pixel size $200 \mu \mathrm{m}$ ). The detector was located $815 \mathrm{~mm}$ from the X-ray source. The object was placed $6.07 \mathrm{~mm}$ from the X-ray source. This resulted in a spatial resolution of $1.50 \mu \mathrm{m}$. A full scan consisted of 750 projections over $360^{\circ}$. The saved projection is an image, obtained in $500 \mathrm{~ms}$ exposure time. GE reconstruction software (Wunstorf, Germany) was used to calculate the 3D structure via back projection. The 3D images, obtained using the v|tome|x XRT, were analysed using Avizo imaging software version 9.2.0.

Average mesh size. Gel mesh size was determined based on the method of Sarkar and coworkers (2015). Calcium-alginate gels $\left(10,20,30 \mathrm{~g} \cdot \mathrm{L}^{-1}\right.$ alginate in $10 \mathrm{mM}$ phosphate buffer $\mathrm{pH} 7.0$ ) were produced in a flat mould (height $\sim 0.5 \mathrm{~cm}$ ) and soaked overnight in $\mathrm{CaCl}_{2}$ solution (5 wt\%) to harden before they were sliced into cylindrical pieces with $2.5 \mathrm{~cm}$ diameter to fit the rheometer probe. These pieces were transferred to fresh $\mathrm{CaCl}_{2}$ solution (5 wt\%) and placed the next day in a rheometer (Physica MCR501, Anton Paar, Graz, Austria) equipped with parallel plate geometry with serrated plates and a diameter of $25 \mathrm{~mm}$ (PP25/P2). The Peltier temperature control system was set at $20^{\circ} \mathrm{C}$ and the normal force at $0.1 \mathrm{~N}$ (various gel thicknesses were used to check for slip). A frequency sweep was performed $\left(10^{-1}\right.$ to $10^{1} \mathrm{~Hz}$ at $0.5 \%$ strain), as well as a strain sweep $(0.1-20 \%$ strain at 0.5 $\mathrm{Hz}$ ). The storage moduli ( $\left.G^{\prime}\right)$ were measured in the linear regime for four independent samples, and used to calculate the average mesh size with Equation 5.1 (Klak, Picard, Giraudier, \& Larreta-Garde, 2012):

$$
\xi^{3}=\frac{\mathrm{k}_{B} T}{G^{\prime}}
$$

Equation 5.1

with $\xi$ the mesh size $(\mathrm{m}), k_{B}$ the Boltzmann constant $\left(1.38 \cdot 10^{-23} \mathrm{~m}^{2} \cdot \mathrm{kg} \cdot \mathrm{K}^{-1} \cdot \mathrm{s}^{-2}\right), T$ the temperature $(293 \mathrm{~K})$ and $G^{\prime}$ the storage modulus $(\mathrm{Pa})$ measured in the linear regime $(1 \mathrm{~Hz}$, $0.5 \%$ strain). 


\section{$\underline{\text { Behaviour under simulated digestive conditions }}$}

Effect of $\mathrm{pH}$ and ionic strength on bead volume. The reference size distribution of the beads was measured first in ultrapure water, using static light scattering (Mastersizer 2000 with Hydro SM dispersion unit, Malvern Instruments, UK). The refractive index of dispersed phase was set at 1.470 (with an absorption index of 0.02) and the refractive index of the dispersant at 1.333. Then, the dispersion unit was filled with a solution of controlled $\mathrm{pH}$ and salt composition. The following solutions were investigated: physiological saline solution (120 mM NaCl, 5 mM KCl, 6 mM CaCl (Kenmogne-Domguia, Meynier, Viau, Llamas, \& Genot, 2012)), saline in the absence of calcium (120 mM NaCl, $5 \mathrm{mM} \mathrm{KCl}$ ), and these two solutions at lower (a factor of 5 and 2, respectively) and higher (3.5 times) ion concentration to systematically study the influence of possible dilution/concentration while passing the GI tract. Phosphate buffers (10 mM) were used at $\mathrm{pH}$ 2.0, 3.0, 4.0, and 7.0, with or without $\mathrm{CaCl}_{2}$ $(6 \mathrm{mM})$. The beads were added to the solution and their size distribution was measured over time (every two min until a plateau was reached or disintegration occurred).

In vitro gastric incubation. Fresh beads were taken from the calcium bath and washed with ultrapure water, using fast ashless filter paper. They were put in simulated gastric fluid consisting of $0.5 \mathrm{~g} \cdot \mathrm{L}^{-1}$ pepsin, $150 \mathrm{mM} \mathrm{NaCl}$ and $10 \mathrm{mM} \mathrm{CaCl} 2$ at $\mathrm{pH} 3.0$ for $2 \mathrm{~h}$ at $37^{\circ} \mathrm{C}$ in a double-walled vessel, and stirred at a rate of $240 \mathrm{rpm}$. The total lipid concentration in the reaction medium was $\sim 1.4 \mathrm{wt} \%$. Undiluted samples were taken from the gastric fluid at 5 , 30, 60 and 110 min, respectively, and analysed by light microscopy.

In vitro intestinal digestion. After $2 \mathrm{~h}$ of gastric incubation, the samples were exposed to static in vitro digestion conditions that represented the intestinal conditions at postprandial (fed) state for $2.5 \mathrm{~h}$. Intestinal components were added to the gastric fluid in the doublewalled vessel $\left(37^{\circ} \mathrm{C}, 240 \mathrm{rpm}\right.$ ), in the order: $10 \mathrm{mM}$ phosphate buffer $\mathrm{pH}$ 7.0, salt stock solution (in $10 \mathrm{mM}$ phosphate buffer $\mathrm{pH}$ 7.0), fresh bile extract stock (in $10 \mathrm{mM}$ phosphate buffer $\mathrm{pH}$ 7.0). The $\mathrm{pH}$ in the vessel was adjusted to $~ 7.05$ using $0.5 \mathrm{M} \mathrm{NaOH}$ or HCL solution, and when the reaction fluid again reached a temperature of $37^{\circ} \mathrm{C}$, fresh pancreatin stock solution (in $10 \mathrm{mM}$ phosphate buffer $\mathrm{pH}$ 7.0) was added to the vessel to start the digestion. The final composition of the digestive fluid was $20 \mathrm{~g} \cdot \mathrm{L}^{-1}$ bile salts, $2.4 \mathrm{~g} \cdot \mathrm{L}^{-1}$ pancreatin, $10 \mathrm{mM} \mathrm{CaCl}_{2}, 150 \mathrm{mM} \mathrm{NaCl}, \sim 0.7 \mathrm{wt} \%$ lipid and $\sim 5 \mathrm{mM}$ phosphate buffer $\mathrm{pH}$ 7.0. The $\mathrm{pH}$ was kept at 7.0 using an automated $\mathrm{pH}$ stat titration unit (877 Titrino Plus titrator, Metrohm, Switzerland) equipped with $100.0 \mathrm{mM} \mathrm{NaOH}$. Lipolysis was measured based on the release of free fatty acids (FFA; see below). Undiluted samples were taken from the intestinal fluid at 15, 30, 50, 100 and $150 \mathrm{~min}$, respectively, and analysed using light microscopy. 
As a reference measurement, the same protocol as described above was used for the intestinal phase, but the simulated gastric fluid was replaced with ultrapure water.

Determination of the free fatty acid (FFA) release. The average actual oil content in the beads was $8.94 \mathrm{wt} \%$. This hardly varied between samples, therefore this value was used in further calculations.

To quantify lipolysis, the FFA content was determined over time ( $\mathrm{Li}, \mathrm{Hu}$, \& McClements, 2011), using the amount of titration fluid (100.0 mM NaOH) needed to keep the pH of the fluid at 7.0, and expressed as the fraction of released FFAs (assuming two FFAs can be released from one triacylglycerol molecule) (Equation 5.2):

$$
F F A=\left(\frac{V_{\mathrm{NaOH}} \cdot M_{\mathrm{NaOH}} \cdot M W_{\text {lipid }}}{\omega_{\text {lipid }} \cdot 2}\right)
$$

Equation 5.2

where $V_{\mathrm{NaOH}}$ and $M_{\mathrm{NaOH}}$ are the volume (L) and molarity $\left(\mathrm{mol} \cdot \mathrm{L}^{-1}\right)$ of sodium hydroxide required to neutralize the sample, $M W_{\text {lipid }}$ the average molecular weight of the lipid material present at the beginning of the experiment (estimated to be $874 \mathrm{~g} \cdot \mathrm{mol}^{-1}$ ), and $\omega_{\text {lipid }}$ the total weight of lipid initially present in the reaction vessel $(\sim 0.3$ g; exact amount determined through oil measurement). The simulated digestive fluid and (empty) alginate beads were titrated to check for any contribution.

\section{Experimental design}

In summary, the beads were made with different sizes (small, S: $0.5-0.6 \mathrm{~mm}$, medium, M: $0.7-0.8 \mathrm{~mm}$ and large, $\mathrm{L}: 1.0-1.2 \mathrm{~mm}$ ) to vary the diffusion distance; and with different mesh sizes (5.4, 6.4 and $9.2 \mathrm{~nm}$ ) to influence the diffusivity of enzymes. For in vitro digestibility, at least two replicates were performed per bead size (S, M, and L) and mesh size, and appropriate blank measurements (four) were done to exclude the influence of the digestive components and alginate matrix on the titratable amount of FFAs. To gain better understanding of the underlying mechanisms, a model was developed based on the bead and mesh size, using FFA release from a non-encapsulated emulsion of known droplet size as a reference for reaction kinetics.

\subsubsection{Model development}

In short, the bead was conceptually divided into 10 concentric layers of equal thickness (see scheme in Supplementary Figure 5.9), which is sufficient to generate a detailed concentration profile as a function of the particle size. The oil distribution at $\mathrm{t}=0$ was assumed based on $\mathrm{X}$-ray tomography. The enzyme concentration in the next time step was calculated using 
Fickian diffusion, and an appropriate time step (10 s) through which the concentration in the next time step was calculated. The following numeric scheme was used (Walstra, 2003):

$$
\begin{gathered}
C(x, t)=\frac{\frac{d m}{d t} \cdot t}{V_{\text {layer }}}+C\left(x, t_{-1}\right)-C_{\text {out } *} \\
C_{\text {out } *}=\left(C\left(x_{+1}, t\right)-C\left(x_{+1}, t_{-1}\right)\right) \cdot \frac{V_{\text {layer } x+1}}{V_{\text {layer } x}} \\
\frac{\mathrm{d} m}{\mathrm{~d} t}=-D_{\text {gel }} \cdot A\left(\frac{\delta c}{\delta x}\right)_{t}
\end{gathered}
$$

where $m$ is the mass (g) of enzyme transported in the direction of the centre of the bead through the cross-sectional area $A\left(\mathrm{~m}^{2}\right)$ of the layer; $V_{\text {layer }}$ is the volume of the layer $\left(\mathrm{m}^{3}\right) ; C$ is the concentration of enzyme $\left(\mathrm{g} \cdot \mathrm{m}^{-3}\right)$, and $C_{\text {out }}$ is the concentration transported to the next layer; $\delta x$ is the layer thickness ( $\mathrm{m} ; 1 / 10$ of the bead radius); and $D_{g e l}\left(\mathrm{~m}^{2} \cdot \mathrm{s}^{-1}\right)$ is the diffusion coefficient of lipase in the alginate gel.

In the second step, the lipolysis rate was calculated following a general equation to describe lipase kinetics $\left(\mathrm{y}=a \cdot \mathrm{x}^{b}\right)$ obtained from non-encapsulated emulsions, using the local enzyme concentration $(C)$ and local substrate concentration (fraction of hydrolysable ester remaining, $H E)$ :

$$
H E(x, t)=C\left(x, t_{-1}\right)-\frac{C(x, t)}{C_{0}} \cdot\left(a \cdot H E\left(x, t_{-1}\right)^{b}\right) \cdot t \quad \text { Equation } 5.6
$$

$$
F F A(x, t)=H E(x, t)-H E\left(x, t_{-1}\right)
$$

Equation 5.7

in which $H E$ is the fraction of hydrolysable ester remaining; $C$ is the concentration of enzyme $\left(\mathrm{g} \cdot \mathrm{m}^{-3}\right)$, and $C_{0}$ is the initial concentration of enzyme $\left(2.4 \cdot 10^{-3} \mathrm{~g} \cdot \mathrm{m}^{-3}\right) ; F F A$ is the fraction of FFA released. The parameters $a$ and $b$ were fitted to FFA release curves obtained with nonencapsulated emulsion of known droplet size (same as used for encapsulation) and equal concentration as used in in vitro digestive conditions $\left(C_{0}\right)$. The parameters were found to be: $a=9.2 \cdot 10^{-4}$ and $b=1.4$. 
In this way, the overall FFA release could be predicted, and through comparison with experimentally determined values, the effective diffusion coefficient of the enzyme could be estimated.

\subsection{RESULTS AND DISCUSSION}

\subsubsection{Production and physical characterisation}

\section{Mesh size of calcium-alginate matrix}

The mesh size of the alginate beads is an important parameter to control enzyme diffusion and hence lipolysis, therefore gels were prepared using different alginate concentrations (Chan \& Neufeld, 2009). The mesh size was determined through rheological measurements (Klak et al., 2012; Sarkar et al., 2015). The elastic (G') modulus increased with increasing alginate concentration (Figure 5.1), indicating a decrease in average mesh size and thus a higher crosslink density. At an alginate concentration of $30 \mathrm{~g} \cdot \mathrm{L}^{-1}$, the average mesh size (5.2 $\pm 0.4 \mathrm{~nm}$ ) is comparable to the size of a lipase complex that has a diameter of about $5.2 \mathrm{~nm}$ (Pignol et al., 2000), while the other mesh sizes are larger but still expected to affect enzyme diffusivity. Please note that these values represent the initial state of the gels.

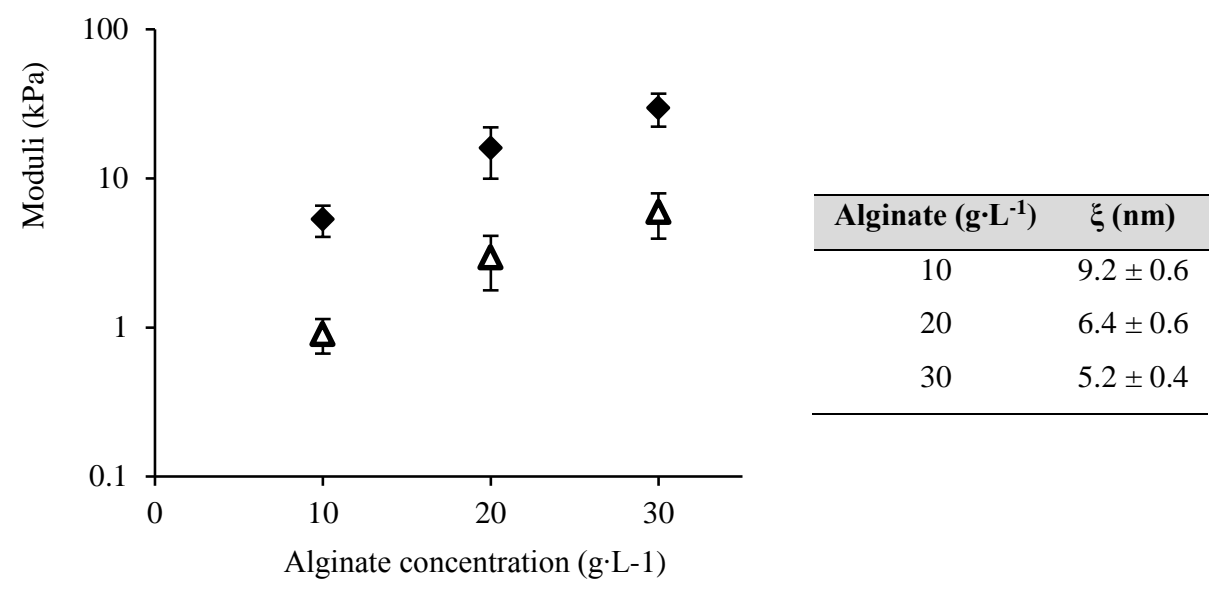

Figure 5.1. Elastic $\left(G^{\prime}, \diamond\right)$ and viscous $\left(G^{\prime},, \Delta\right)$ moduli of calcium-alginate gels, as a function of alginate concentration, measured in the linear viscoelastic regime (frequency of $1 \mathrm{~Hz}, 0.5 \%$ strain), and the corresponding calculated average mesh size $\xi$ (average \pm SD; $n=4$ ).

Bead production, appearance and size

Figure 5.2a shows emulsion-filled alginate beads of different sizes. The bead size was very well controlled through production conditions, as was described in Workamp et al. (2016). 
In Figure 5.2b the typical microscopic appearance of the beads is shown, with the trapped emulsion droplets clearly visible within the gel. Figure 5.2c shows a 3D image of a bead (8.9 $w t \%$ oil, $20 \mathrm{~g} \cdot \mathrm{L}^{-1}$ alginate, size $\mathrm{M}$ ) from which it can be seen that the oil droplets were homogeneously partitioned throughout the bead, only having a lower oil content in the outer shell since no 'half' oil droplets are encapsulated in the shell. Such a partitioning was taken into account when further developing the model describing lipolysis kinetics.

The microscopic appearance and bead size remained constant for at least $50 \mathrm{~d}\left(4^{\circ} \mathrm{C}, 5 \mathrm{wt} \%\right.$ $\mathrm{CaCl}_{2}$, data not shown). Such a high physical stability was expected due to the strong crosslinks of calcium-alginate gel (Romo \& Perez-Martinez, 1997).

a.

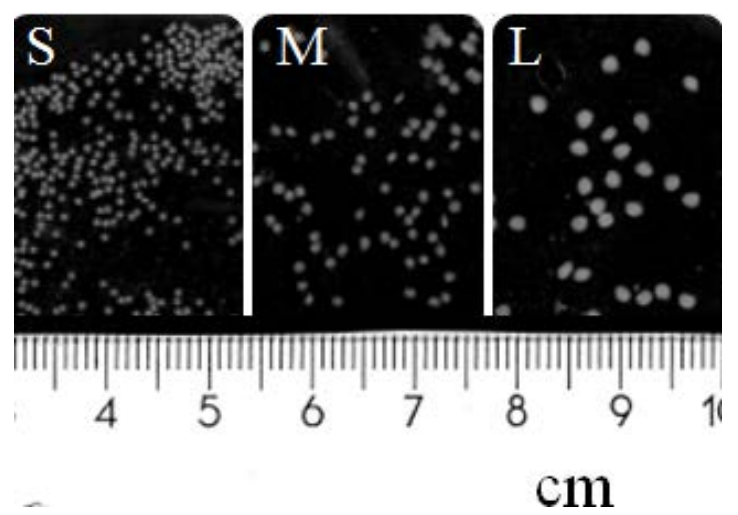

b.

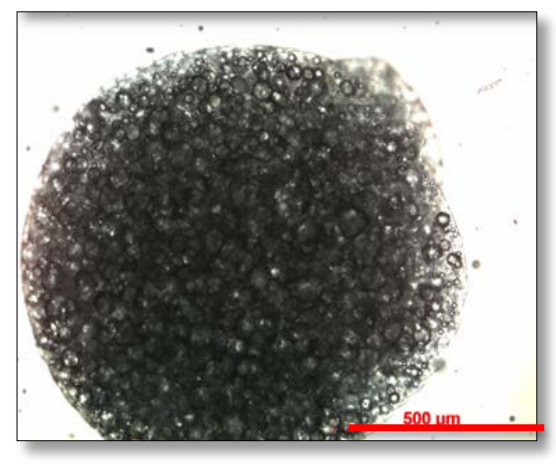

c.

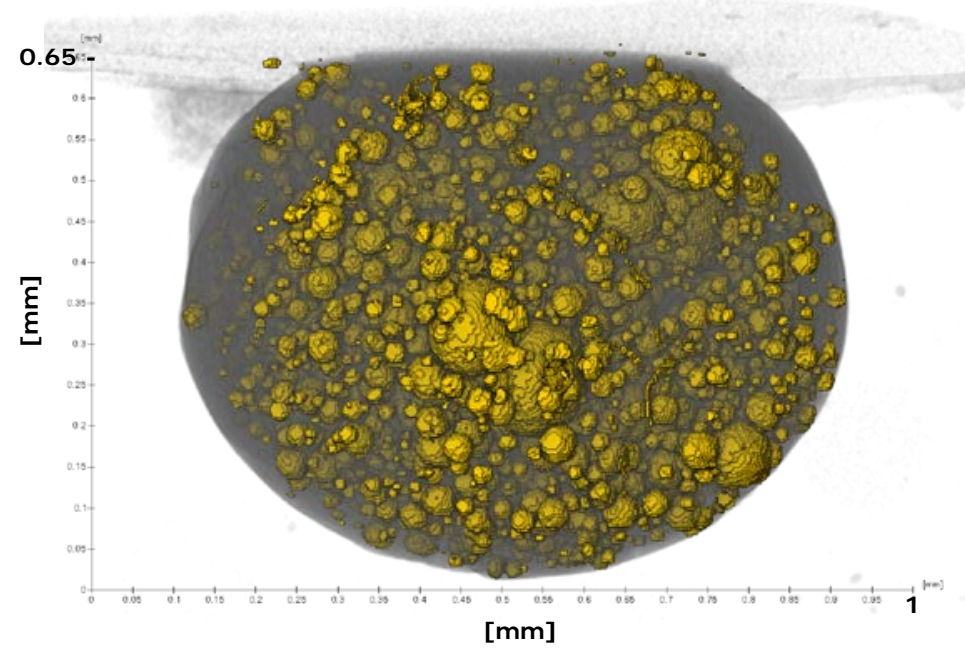

Figure 5.2. Visual appearance of emulsion-filled alginate beads (8.9 wt\% oil, $10 \mathrm{~g} \cdot \mathrm{L}^{-1}$ alginate in the aqueous phase), a) normal camera images with cm scale; b) light microscopic image, red scale bar represents $500 \mu \mathrm{m}$, and c) 3D image using X-ray tomography. 


\subsubsection{Influence of $\mathrm{pH}$ and ionic conditions on the physical characteristics of the beads}

Since alginate gel is based on cross-links between divalent calcium ions and deprotonated carboxylic acid groups of guluronic acid residues, it is sensitive to the ionic strength and $\mathrm{pH}$ of the environment. In food and in the GI tract, various conditions are encountered that may affect the behaviour of the gel beads, i.e., they may swell, shrink or even dissolve ( $\mathrm{Li}, \mathrm{Hu}$, Du, et al., 2011; Rayment et al., 2009; Zeeb, Saberi, et al., 2015). Therefore bead appearance and size was recorded for conditions relevant to the GI tract, both in terms of ionic composition (section 3.2.1) and $\mathrm{pH}$ (section 3.2.2).

Influence of ions on the appearance and size of the beads

Emulsion-filled alginate beads ( $8.9 \mathrm{wt} \%$ oil; $20 \mathrm{~g} \cdot \mathrm{L}^{-1}$ alginate in the aqueous phase; $0.7 \mathrm{~mm}$ ) were exposed to salt solutions (in presence and absence of calcium) that are relevant to human physiological conditions: $120 \mathrm{mM} \mathrm{NaCl}, 6 \mathrm{mM} \mathrm{CaCl}_{2}$ and $5 \mathrm{mM} \mathrm{KCl}$ (Kenmogne-Domguia et al., 2012). In the absence of environmental calcium (Figure 5.3, ratio 0:125), the beads were greatly affected by the ionic strength, showing swelling and possibly disintegration as was observed by others (Bajpai \& Sharma, 2004; Mahdavinia, Rahmani, Karami, \& Pourjavadi, 2014). This is probably caused by rapid $\mathrm{Na}^{+}-\mathrm{Ca}^{2+}$ exchange process, therewith removing the egg-box bonds (Stewart et al., 2014) and allowing the gel to swell and soften (Bajpai \& Sharma, 2004; LeRoux, Guilak, \& Setton, 1999). At higher total ion concentration, swelling occurred faster, which is in line with findings for alginate-shell capsules (Rolland, Santanach-Carreras, Delmas, Bibette, \& Bremond, 2014). Swelling makes the beads mechanically less strong, so less resistant against the capillary force exerted by the cover slip. This allows the bead to break upon microscope slide preparation, but it has to be noted that the observed broken structure (e.g., Figure 5.3, $65.5 \mathrm{mM}$ monovalent cations, ratio 0:125) is not a typical result of overall disintegration in fluid.

At physiological concentration of monovalent cations, the presence of calcium greatly affected the bead behaviour (Figure 5.3, $125 \mathrm{mM}$ monovalent cation). Disintegration was already prevented at low concentration ( $3 \mathrm{mM}$ calcium) and the swelling was reduced when increasing calcium concentration. At physiological calcium concentration (6 mM), the beads swelled slightly (24\% volume change), indicating that some calcium bridges (between M-M and M-G blocks) may have been broken, but that the strongest 'egg-box' bonds remained intact (Donati et al., 2005). At even higher calcium concentration (21 mM), the bead volume hardly changed (6\% volume reduction).

When keeping the physiological ratio of calcium to monovalent cations constant (Figure 5.3, ratio 6:125), the total ionic concentration hardly affected bead appearance. This indicates that the ratio of calcium to monovalent cations is above the critical value for ion exchange and 
disintegration (LeRoux et al., 1999; Velings \& Mestdagh, 1995). The bead volume only increased slightly (up to 37\%) at the lowest total concentration tested. This can most probably be assigned to less screening and therefore more electrostatic repulsion between alginate chains (Velings \& Mestdagh, 1995), in combination with a reduction of cross-links allowing the network to swell (Bajpai \& Sharma, 2004).

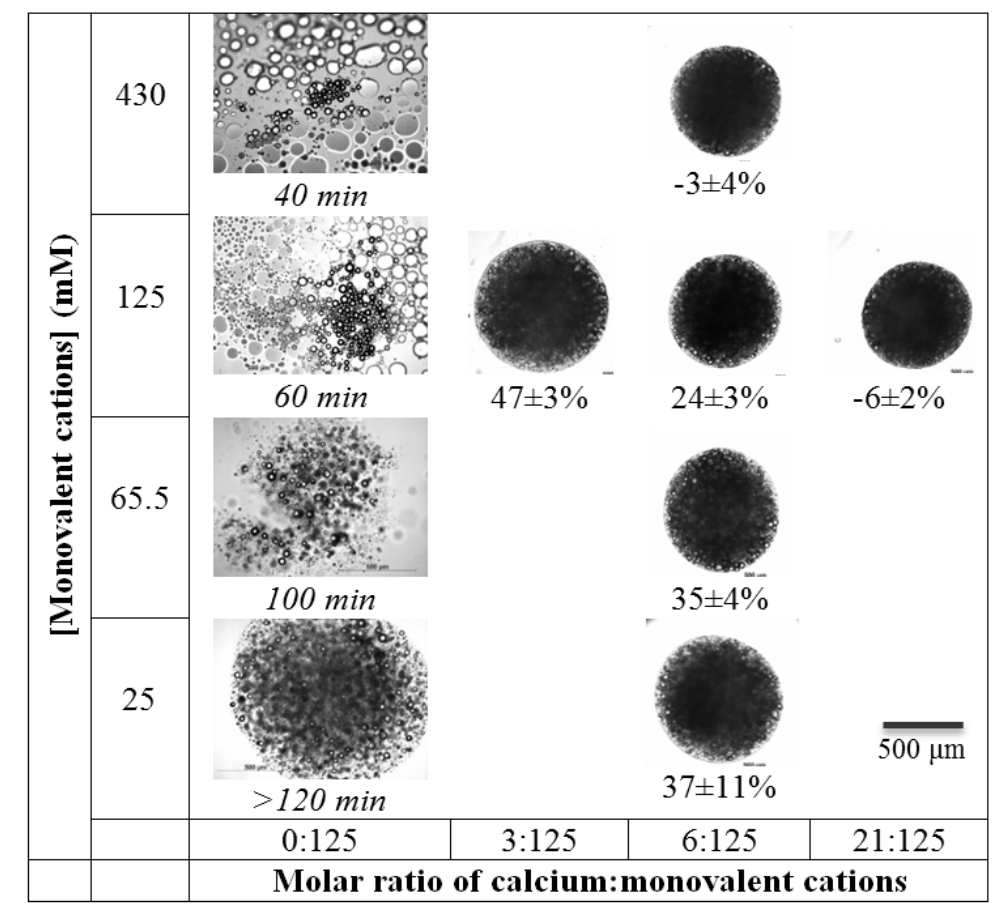

Figure 5.3. Effect of various ion solutions on microscopic appearance of emulsion-filled alginate beads (after $2 \mathrm{~h}$ incubation), and maximum volume change of the beads (\%) for beads that reached a maximum volume without disintegration under shear (average $\pm \mathrm{SD} ; n=3$ ). Beads incubated without calcium disintegrated at the indicated times.

\section{Influence of $\mathrm{pH}$ on the appearance and size of the beads}

Throughout the human GI tract, a range of $\mathrm{pH}$ conditions occur, including acidic $\mathrm{pH}$ in the stomach ( $\mathrm{pH}$ 2.0-4.0) and neutral $\mathrm{pH}$ in the small intestine ( $\mathrm{pH}$ 7.0). To study $\mathrm{pH}$ dependency of bead appearance and size, emulsion-filled alginate beads $\left(20 \mathrm{~g} \cdot \mathrm{L}^{-1}\right.$ alginate; $\left.0.7 \mathrm{~mm}\right)$ were exposed to a range of buffers $(10 \mathrm{mM}$ phosphate buffers $\mathrm{pH} 2.0-7.0$, in the absence and presence of calcium). Figure $\mathbf{5 . 4}$ shows that the beads remained intact at $\mathrm{pH} 2.0$ and shrank slightly. At higher $\mathrm{pH}$, the beads swelled considerably, which is in line with literature ( $\mathrm{Li}$, Hu, Du, et al., 2011; Rayment et al., 2009; Zeeb, Saberi, et al., 2015). Below their $\mathrm{pK}_{\mathrm{a}}(\sim 3.5$ 
for alginate (Li, Hu, Du, et al., 2011; Mahdavinia et al., 2014)), carboxyl acid groups become protonated and calcium ions dissociate from the gel, changing the network from an ionic gel towards an acid gel based on hydrogen bonds (Norton, Frith, \& Ablett, 2006). Around or above the $\mathrm{pK}_{\mathrm{a}}$, less carboxyl groups are protonated so repulsive interactions result in swelling (Mahdavinia et al., 2014; Rayment et al., 2009), and changes in surface charge (Zeeb, Saberi, et al., 2015).

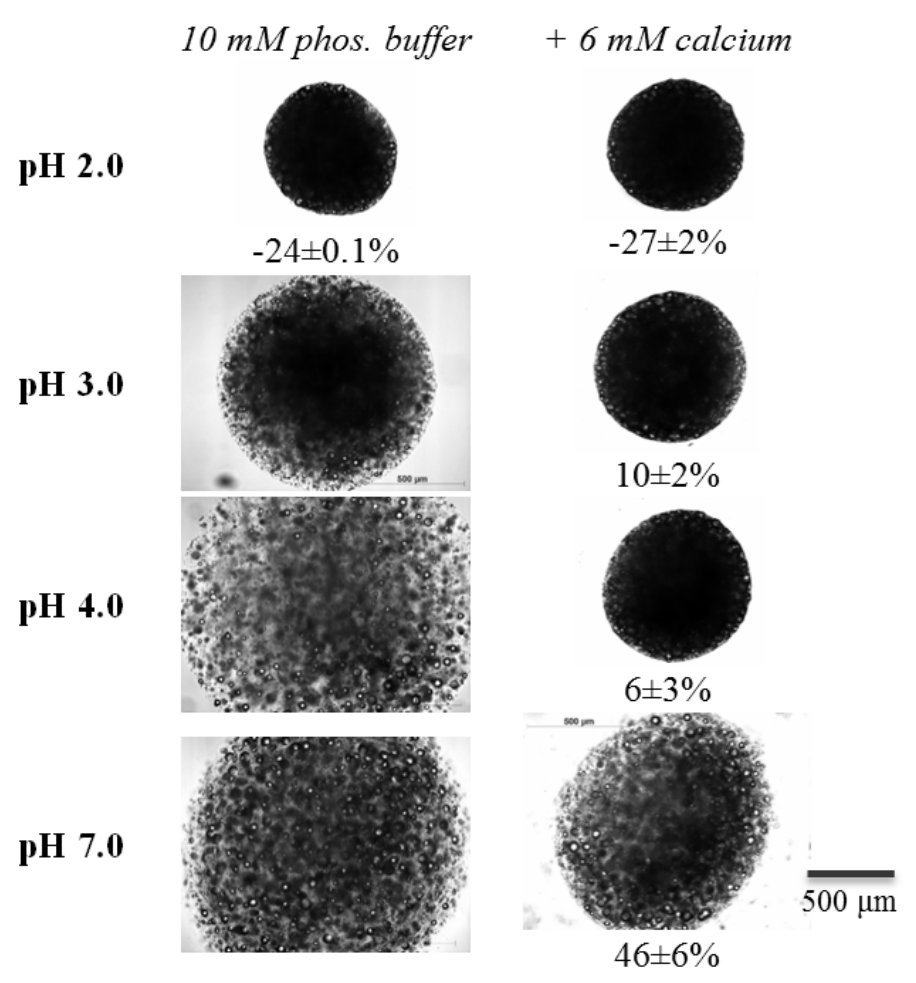

Figure 5.4. Effect of $\mathrm{pH}$ on microscopic appearance of emulsion-filled alginate beads (after $2 \mathrm{~h}$ ) and maximum volume change of the beads (\%) for beads that reached a maximum volume without disintegration under shear (average $\pm \mathrm{SD} ; n=3)$. Phosphate buffers $(10 \mathrm{mM})$ were used in presence or absence of $6 \mathrm{mM}$ added calcium.

In the absence of added calcium, disintegration occurred at $\mathrm{pH} 3.0$ to $\mathrm{pH} 7.0$ (Figure 5.4, left column), as also seen in Figure 5.3 ( $\mathrm{pH} \sim 5-6$ ), which may be a result of extraction of incorporated calcium from the network, via exchange with $\mathrm{Na}^{+}$and precipitation by phosphate (Song, Hahn, \& Hoffmann, 2002). When $6 \mathrm{mM}$ calcium was added, bead stability improved (Figure 5.4, right column); at low $\mathrm{pH}$, the appearance hardly changed, but at $\mathrm{pH}$ 7.0 swelling was observed due to calcium precipitation and the beads disintegrated completely over a few days. 
In summary, these findings provide clear indications for the use of calcium-alginate gel beads as delivery vehicles. At low calcium concentration, the beads remain intact in acidic conditions (as present in the stomach), while they will start to swell and disintegrate at higher $\mathrm{pH}$ values (more distal parts of the intestine). Bead integrity can be assured for storage when combined with acidic food rich in calcium, such as yogurt.

\subsubsection{In vitro gastrointestinal digestion}

\section{Bead appearance during simulated gastrointestinal digestion}

Bead appearance was investigated during simulated gastric and intestinal digestion (Figure 5.5). During gastric incubation, none of the beads were substantially affected in terms of bead appearance and emulsion droplet size, and all beads resisted the capillary forces between the microscopic slide and cover glass that are known to squash fragile beads. This is in line with our findings for the behaviour of the beads at $\mathrm{pH} 3.0$ in the presence of calcium (Figure 5.4).

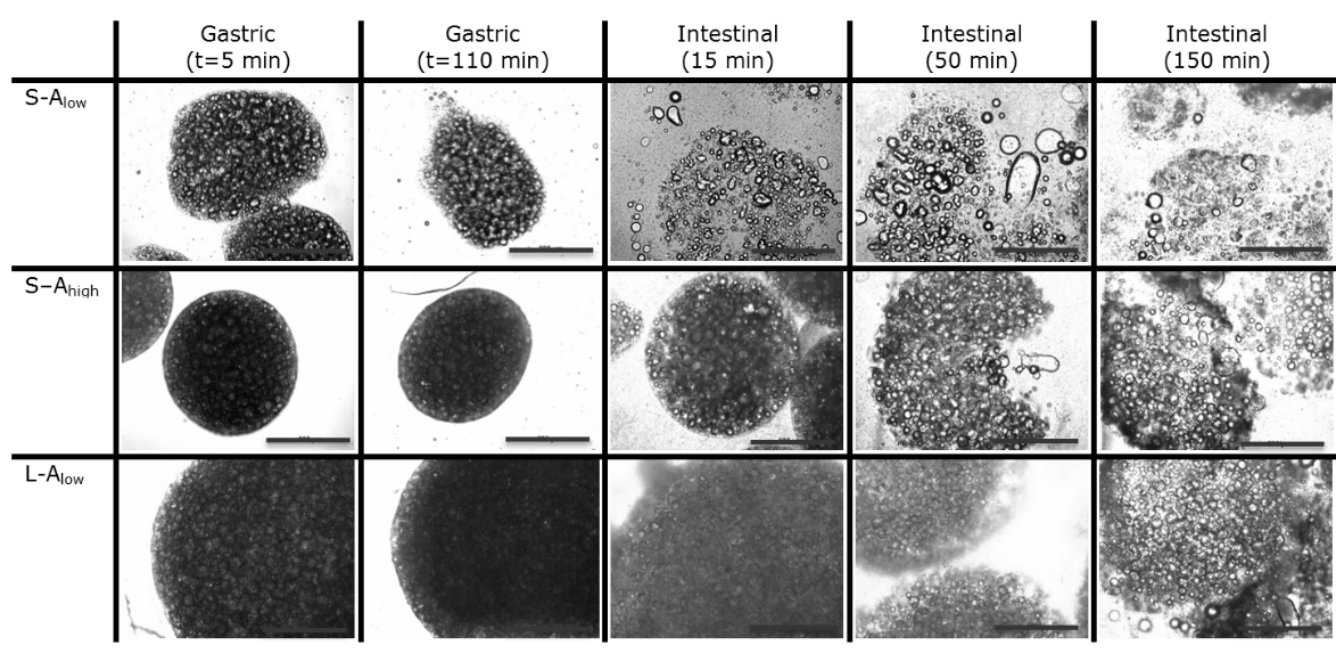

Figure 5.5. Light microscopy images of beads during gastric ( $\mathrm{t}=5$ and $110 \mathrm{~min}$ ) and intestinal incubation ( $\mathrm{t}=15,50$ and $150 \mathrm{~min}$ ). The scale bar represents $500 \mu \mathrm{m}$. Abbreviations: $\mathrm{S}=$ small beads $(0.5-0.6 \mathrm{~mm}), \mathrm{L}=$ large beads $(1.0-1.2 \mathrm{~mm}), A_{\text {low }}$ and $A_{\text {high }}=$ low and high alginate concentration (10 and $30 \mathrm{~g} \cdot \mathrm{L}^{-1}$, respectively). Per time point, three images were taken and a representative example is shown here.

During intestinal incubation, the overall microstructure of the beads clearly changed and depended on bead type. Already after 15 min of intestinal incubation, the microstructure of all beads showed a more loose structure, as has been mentioned in the literature (Rayment et al., 2009; Wright et al., 2009). Small beads with large mesh size (S-A $\left.\mathrm{A}_{\text {low }}\right)$ were already disrupted after 15 min of intestinal incubation, while small beads with lower matrix mesh 
size (S- $\mathrm{A}_{\text {medium }}$ and $\mathrm{S}-\mathrm{A}_{\text {high }}$ ) remained intact longer. Medium and large sized beads also took more time to swell compared to small beads. These findings consistently point to diffusiondriven processes. It has to be noted that the observed broken structures are not representing disintegration in fluid, but are due to capillary forces exerted by the microscope slide.

\section{Effect of the gastric phase on in vitro intestinal lipolysis}

From the experiments described above we expected that the gastric phase would not greatly influence intestinal lipolysis. In Figure 5.6 the FFA release from small and large beads prepared with $20 \mathrm{~g} \cdot \mathrm{L}^{-1}$ alginate is shown as a function of intestinal incubation time with and without prior gastric phase, together with that for a non-encapsulated emulsion (without prior gastric phase to have the same initial droplet size as that of the encapsulated emulsion). The overall digestive behaviour of the beads is very different from that of conventional whey protein-stabilized emulsions: in the latter, oil is easily accessible for lipase so lipolysis is fast, whereas lipase diffusion is slowed down by the hydrogel matrix, leading to delayed lipolysis.

When a gastric phase was applied prior to the intestinal phase, the induction period (time after which intestinal digestion starts) seemed slightly prolonged; yet it should be pointed out that the observed difference is rather small compared to the experimental variability, making it difficult to unambiguously draw conclusions regarding this effect.

a.

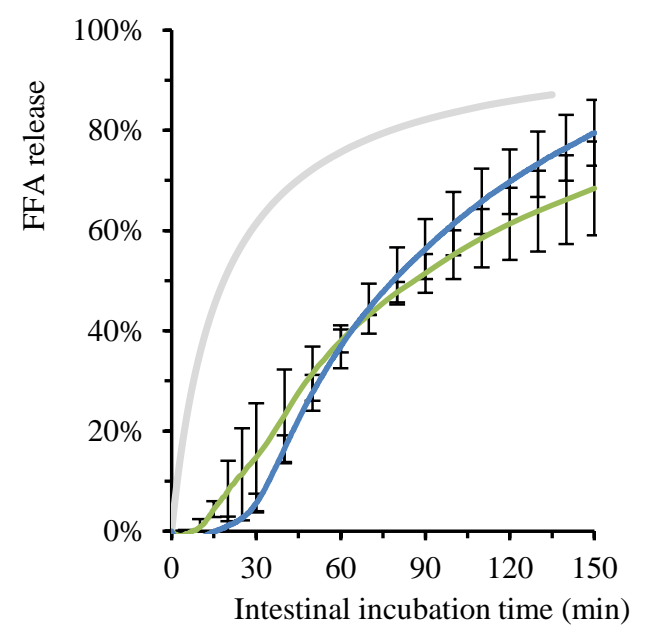

b.

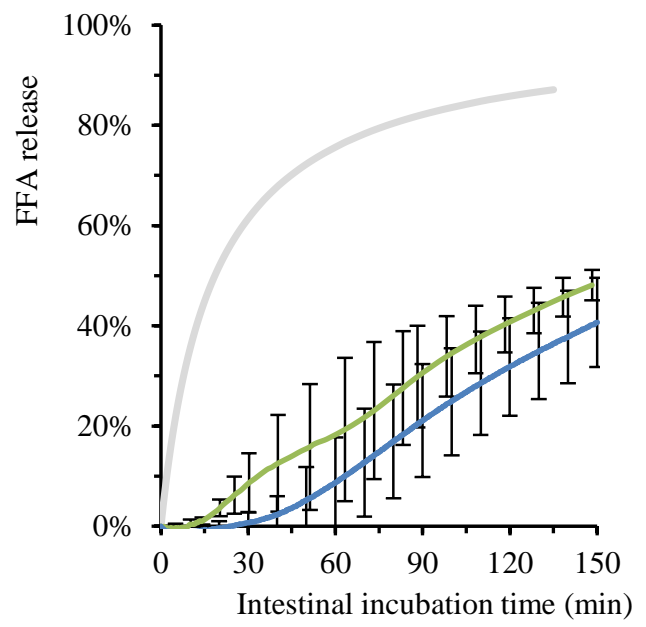

Figure 5.6. Free fatty acid (FFA) release during incubation in simulated intestinal fluid that represents the postprandial state (20 g. $\mathrm{L}^{-1}$ bile; $2.4 \mathrm{~g} \cdot \mathrm{L}^{-1}$ lipase), with (blue lines) or without (green lines) prior gastric incubation, using emulsion-filled alginate beads (prepared with $20 \mathrm{~g} \cdot \mathrm{L}^{-1}$ ) with a) small size $(0.5-0.6 \mathrm{~mm})$ and $b$ ) large size $(1.0-1.2 \mathrm{~mm})$ (average $\pm S D ; n \geq 2)$. The grey line indicates the intestinal digestion pattern of a WPI-stabilized reference emulsion without prior gastric phase. 


\section{Effect of bead design on lipolysis kinetics}

Figure 5.7 shows the FFA release from emulsion-filled alginate beads prepared with different alginate concentrations over time for different bead sizes, during in vitro intestinal incubation after prior gastric phase. In general, the FFA release profiles of emulsion-filled alginate beads showed an induction period (time after which digestion starts) followed by a typical lipolysis curve. Such an induction period has also been observed by others for indigestible gel matrices (Sarkar et al., 2015). As expected, the lipolysis rate was a function of the bead and mesh sizes, with larger beads (longer diffusion distance) and smaller mesh sizes (slower diffusion) leading to slower FFA release. At an average mesh size comparable to the size of lipase $\left(5.2 \mathrm{~nm} ; 30 \mathrm{~g} \cdot \mathrm{L}^{-1}\right.$ alginate), the induction period was long, and lipolysis was much slower compared to gels with two-fold larger mesh size (9.2 nm; $10 \mathrm{~g} \cdot \mathrm{L}^{-1}$ alginate).
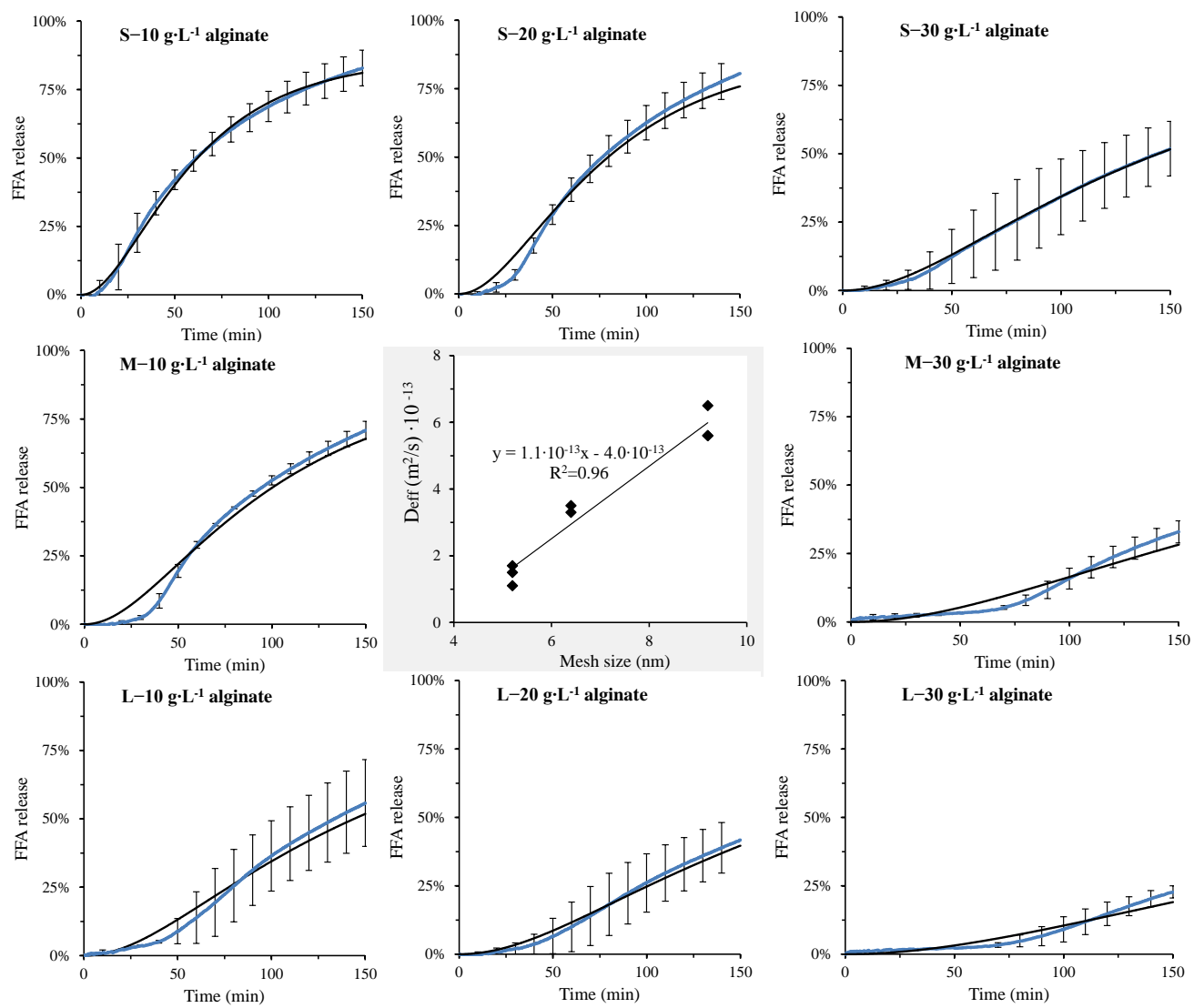

Figure 5.7. Free fatty acid (FFA) release during incubation in simulated intestinal fluid that represents the postprandial state ( $20 \mathrm{~g} \cdot \mathrm{L}^{-1}$ bile; $2.4 \mathrm{~g} \cdot \mathrm{L}^{-1}$ lipase), after gastric incubation, from emulsion-filled alginate beads prepared with a range of alginate concentrations (i.e., mesh sizes) and bead sizes. Blue lines represent average $\pm S D ; n \geq 2$ ); black lines indicate the modelled FFA release. The fitted effective diffusion coefficients $\left(D_{\text {eff }}\right)$ are plotted in the middle panel as function of mesh size. 
Li et al. (2011) also varied alginate concentration (0.25; 0.5; $1 ; 2 \mathrm{w} / \mathrm{v} \%)$ to make emulsionfilled beads, and suggested the slower rate of intestinal lipolysis to be at least partly be attributed to the formation of a denser gel network that delays lipase movement. No induction period was observed in their in vitro intestinal lipolysis profile ( $\mathrm{Li}, \mathrm{Hu}, \mathrm{Du}$, et al., 2011), which points again to the influence of a prior gastric phase.

As a next step, a model was used to estimate an effective diffusion coefficient of the enzyme (Figure 5.7, middle panel). The obtained values that were found in the beads are much lower ( 30-180 times) than diffusion in aqueous solutions (estimated to be $\sim 2 \cdot 10^{-11} \mathrm{~m}^{2} \cdot \mathrm{s}^{-1}$ ). And the effective diffusion coefficient linearly varied with the initial mesh size, when overlapping the values for beads of different sizes.

The observed induction period was overlooked in the model, and can partly be attributed to an initial adaptation from shrinkage in the prior gastric phase. Besides, it has to be considered that this observed induction period may point to a slight difference in the structure of the outer shell of the beads. This is somehow taken into account in the model, with a lower oil content in the external bead layer, but in addition it may also be affected by a smaller mesh size in the outer shell compared to the core (Wright et al., 2009). Active water transport during swelling could influence enzyme transport as well, reducing the induction period. However, the actual diffusion distance is not affected that much (i.e., a typical volume change of $46 \%$ leads to a diameter change of $12 \%$ ), so we expect the active water transport to have negligible overall effects. Another effect that is not covered in the model is the structural changes in the beads subjected to digestive conditions: the beads swell slightly at neutral $\mathrm{pH}$ in presence of calcium and lose mechanical strength, but remain intact in the intestinal fluid in contrast to breaking between microscope slides. In the effective diffusion coefficient, these swelling effects are combined with the diffusion coefficient into an overall value for the resistance against diffusion. Still, these overall values (Figure 5.7, middle panel) are believed to be indicative of the diffusion process as swelling is restricted in the presence of calcium.

Although many additional refinements of the model could be made, such as a change in mesh size as function of time and locally adjusted diffusion coefficients, the fact that the model is able to describe the overall behaviour makes it a valuable tool to design beads for targeted delivery. With the equation that describes diffusion rate as function of mesh size (Figure 5.7, middle panel), effects can be predicted based on mesh size and diffusion distance (i.e., bead size) as done in the design diagrams of Supplementary Figure 5.10. The diagrams clearly show that a range of bead size-mesh size combinations can be used to target a certain release, for example, 50\% FFA release after $120 \mathrm{~min}$. The diagrams also show that at small mesh size, a change in bead size has a large impact; and that at small bead size, a change in mesh size has a small impact. This knowledge will facilitate designing beads for controlled delivery 
purposes. In concert with the findings on bead stability in the presence of calcium, this allows us to take the next step towards dynamic in vitro digestion experiments, and eventually human clinical trials to determine the effect of ingesting beads on satiety and food intake.

\subsection{CONCLUSIONS}

Our objective was to encapsulate emulsion droplets in alginate beads, and systematically investigate the suitability of these beads for controlled in vitro intestinal delivery. Emulsionfilled alginate beads were successfully produced with a range of bead and mesh sizes. Their physical characteristics were found to be largely affected by $\mathrm{pH}$ and ionic concentrations. All emulsion-filled alginate beads had excellent gastric stability, but showed some swelling under simulated intestinal conditions, and could even disintegrate with insufficient calcium concentration (i.e., if calcium is chelated or bound to, for example, a biopolymer).

Through variation of bead and mesh size, a broad range of in vitro lipolysis profiles was obtained that greatly differed from that of non-encapsulated emulsions, with longer induction period and slower kinetics at increasing bead size and decreasing mesh size. From modelling studies, we obtained an equation linking lipase diffusion to lipolysis rates, which is important input for the design of beads with a specific release profile. Although our findings need to be validated in vivo, we consider these beads as an essential step towards ingested lipids for release in the distal small intestine, which could be part of appetite control and weight management strategies.

\section{Acknowledgement}

Research presented in this publication was financially supported by the Graduate School VLAG. We thank Joshua Dijksman and Marcel Workamp for providing the nozzle holder. Besides, we thank Remco Hamoen for his help with X-ray tomography; the X-ray tomography equipment is owned by Shared Research Facilities and subsidized by Ministry of Economic Affairs and the province of Gelderland, The Netherlands. We also thank JanEise Vuist for his help with the design diagrams. 


\subsection{SUPPLEMENTARY INFORMATION}

Emulsion-filled alginate beads were produced through the injection method shown in Figure 5.8a, using the nozzle design described in (Workamp et al., 2016). The alginate-emulsion feed formed a pendant droplet on the nozzle that grew till it detached, and fell into a calcium bath to solidify and form a bead. Drop detachment and the influence of the drag force generated by the air flow, which adds to the weight pulling the droplet from the nozzle, is described in detail in (Workamp et al., 2016). In Figure 5.8b, the relation between applied pressure and bead size is shown.

a.

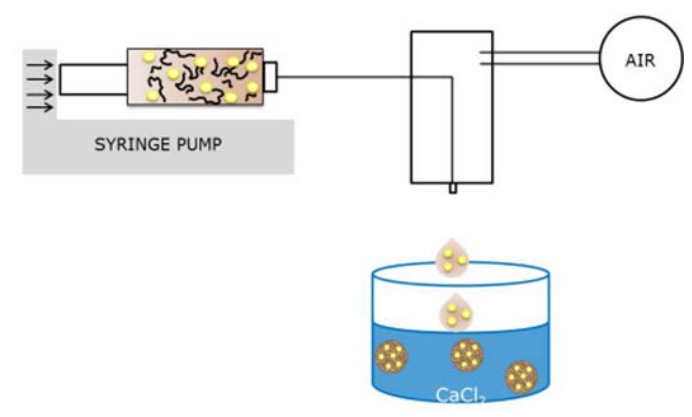

b.

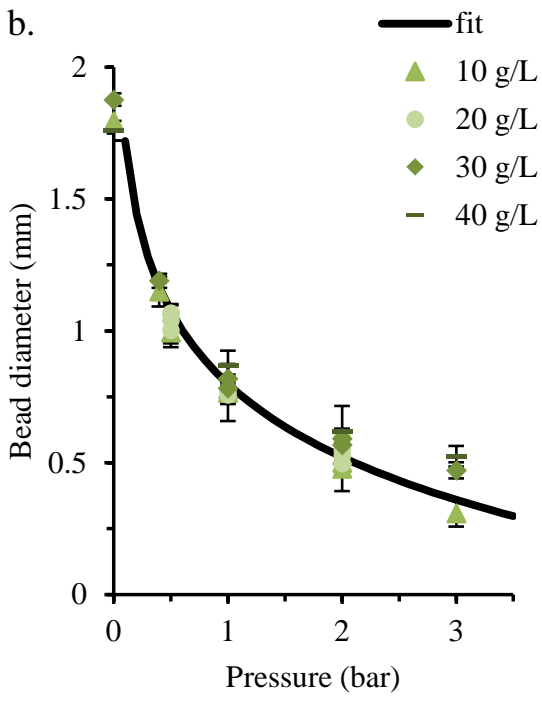

Supplementary Figure 5.8. a) Bead production set-up and b) relation between pressure of the air flow and bead size, using the four indicated alginate concentrations. All beads contained $8.9 \mathrm{wt} \%$ oil; the feed flow was $1 \mathrm{~mL} / \mathrm{min}$. The data points represent average values of at least 50 beads, with standard deviation. 


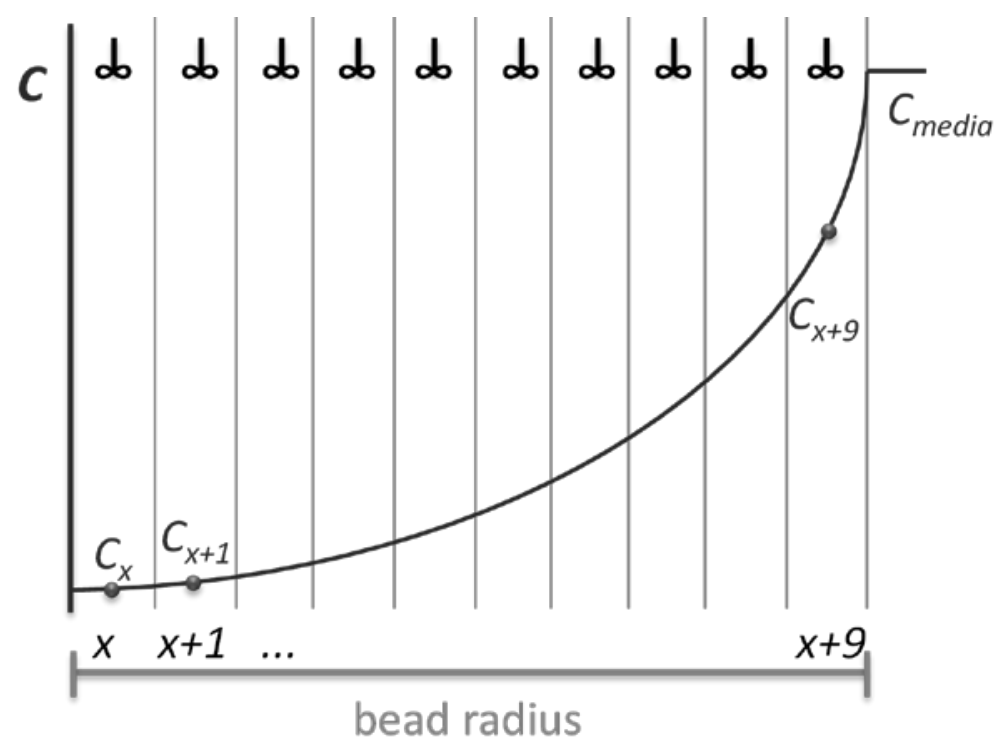

Supplementary Figure 5.9. Scheme of the concentric layers of equal thickness (x) and corresponding enzyme concentrations (C) used in the model. 


\section{Design diagrams}
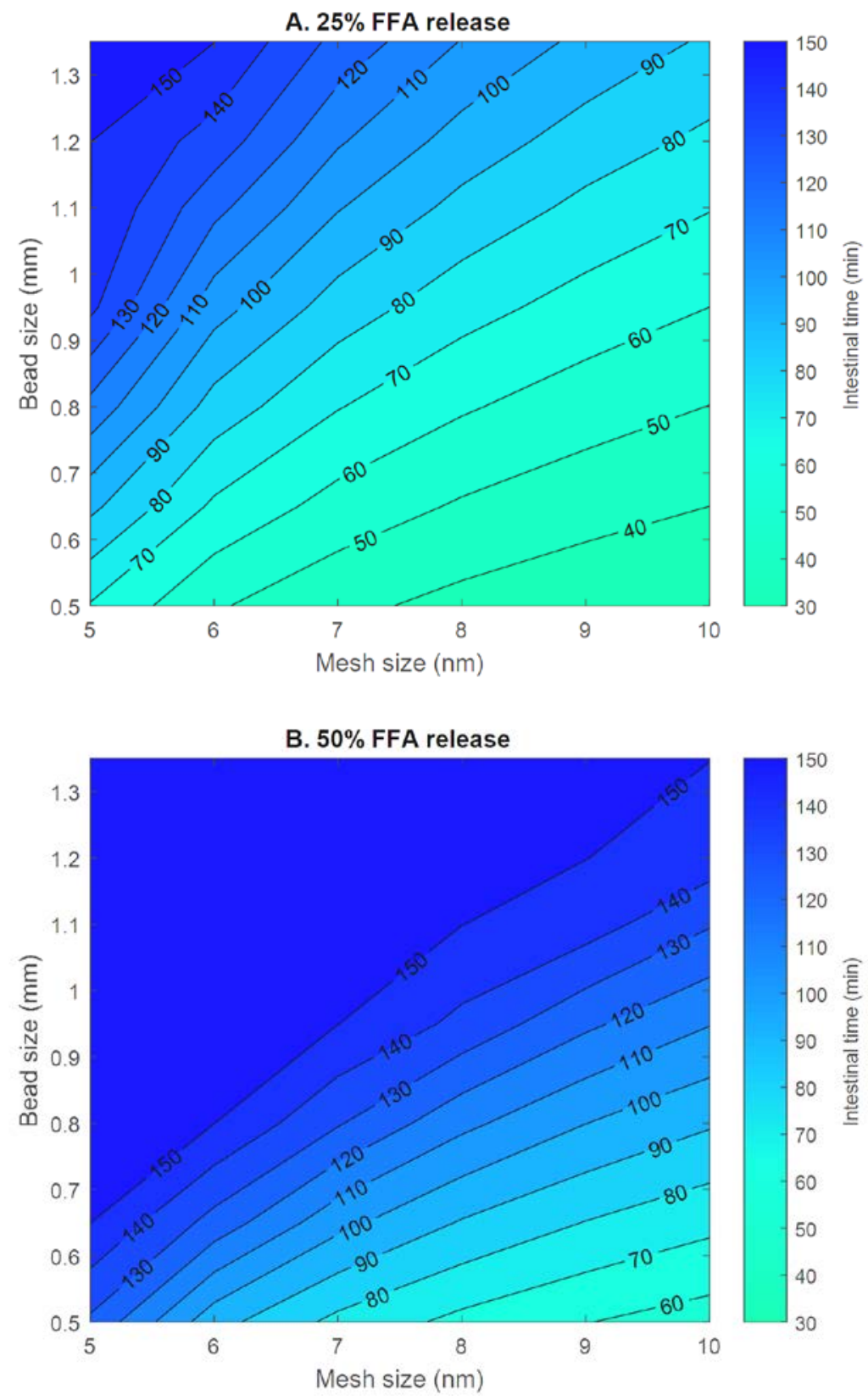

Supplementary Figure 5.10. Design diagrams of predicted intestinal time to reach A. $25 \%$ and B. $50 \%$ FFA release as function of bead and mesh size, in emulsion-loaded calcium-alginate gel beads. 


\subsection{REFERENCES}

Alleleyn, A. M. E., van Avesaat, M., Troost, F. J., \& Masclee, A. A. M. (2016). Gastrointestinal Nutrient Infusion Site and Eating Behavior: Evidence for A Proximal to Distal Gradient within the Small Intestine? Nutrients, 8(3), 117-132.

Arica, B., Çaliş, S., Ati̇lla, P., Durlu, N. T., Çakar, N., Kaş, H. S., \& Hincal, A. A. (2005). In vitro and in vivo studies of ibuprofen-loaded biodegradable alginate beads. Journal of Microencapsulation: Micro and Nano Carriers, 22(2), 153-165.

Bajpai, S. K., \& Sharma, S. (2004). Investigation of swelling/degradation behaviour of alginate beads crosslinked with $\mathrm{Ca} 2+$ and Ba2+ ions. Reactive and Functional Polymers, 59(2), 129-140.

Bakala N’Goma, J.-C., Amara, S., Dridi, K., Jannin, V., \& Carrière, F. (2012). Understanding the lipiddigestion processes in the GI tract before designing lipid-based drug-delivery systems. Therapeutic Delivery, 3(1), 105-124.

Brownlee, I. A., Allen, A., Pearson, J. P., Dettmar, P. W., Havler, M. E., Atherton, M. R., \& Onsøyen, E. (2005). Alginate as a source of dietary fiber. Critical Reviews in Food Science and Nutrition, 45(6), 497-510.

Chan, A. W., \& Neufeld, R. J. (2009). Modeling the controllable pH-responsive swelling and pore size of networked alginate based biomaterials. Biomaterials, 30(30), 6119-29.

Chu, B.-S., Rich, G. T., Ridout, M. J., Faulks, R. M., Wickham, M. S. J., \& Wilde, P. J. (2009). Modulating pancreatic lipase activity with galactolipids: effects of emulsion interfacial composition. Langmuir, 25(16), 9352-9360.

Corstens, M. N., Berton-Carabin, C. C., de Vries, R., Troost, F. J., Masclee, A. A. M., \& Schroën, K. (2017a). Food-grade micro-encapsulation systems that may induce satiety via delayed lipolysis: A Review. Critical Reviews in Food Science and Nutrition, 57(10), 2218-2244.

Corstens, M. N., Berton-Carabin, C. C., Kester, A., Fokkink, R., van den Broek, J. M., de Vries, R., ... Schroën, K. (2017b). Destabilization of multilayered interfaces in digestive conditions limits their ability to prevent lipolysis in emulsions. Food Structure, 12, 54-63.

Dekkers, B., Kolodziejczyk, E., Acquistapace, S., Engmann, J., \& Wooster, T. J. (2016). Impact of gastric $\mathrm{pH}$ profiles on the proteolytic digestion of mixed $\beta 1 \mathrm{lg}$-Xanthan biopolymer gels. Food Function, 7(1), 58-68.

Donati, I., Holtan, S., Mørch, Y. A., Borgogna, M., Dentini, M., \& Skjåk-Bræk, G. (2005). New hypothesis on the role of alternating sequences in calcium-alginate gels. Biomacromolecules, 6(2), 1031-1040.

Golding, M., Wooster, T. J., Day, L., Xu, M., Lundin, L., Keogh, J., \& Clifton, P. (2011). Impact of gastric structuring on the lipolysis of emulsified lipids. Soft Matter, 7, 3513-3523.

Grant, G. T., Morris, E. R., Rees, D. A., Smith, P. J. C., \& Thom, D. (1973). Biological interactions between polysaccharides and divalent cations: the egg-box model. FEBS Letters, 32(1), 195-198.

Hu, M., Li, Y., Decker, E. A., Xiao, H., \& McClements, D. J. (2011). Impact of Layer Structure on Physical Stability and Lipase Digestibility of Lipid Droplets Coated by Biopolymer Nanolaminated Coatings. Food Biophysics, 6, 37-48.

Kenmogne-Domguia, H. B., Meynier, A., Viau, M., Llamas, G., \& Genot, C. (2012). Gastric conditions control both the evolution of the organization of protein-stabilized emulsions and the kinetic of lipolysis during in vitro digestion. Food \& Function, 3(12), 1302-9.

Klak, M.-C., Picard, J., Giraudier, S., \& Larreta-Garde, V. (2012). Mastered proteolysis of gelatin gel can control delivery kinetics of entrapped large molecules. Soft Matter, 8(17), 4750.

Klinkesorn, U., \& McClements, D. J. (2010). Impact of lipase, bile salts, and polysaccharides on properties and digestibility of tuna oil multilayer emulsions stabilized by lecithin-chitosan. Food Biophysics, 5(2), 73-81.

LeRoux, M. A., Guilak, F., \& Setton, L. A. (1999). Compressive and shear properties of alginate gel: Effects of sodium ions and alginate concentration. Journal Biomedical Material Research, 47(November), 4653.

Li, Y., Hu, M., Du, Y., Xiao, H., \& McClements, D. J. (2011). Control of lipase digestibility of emulsified lipids by encapsulation within calcium alginate beads. Food Hydrocolloids, 25(1), 122-130.

Li, Y., Hu, M., \& McClements, D. J. (2011). Factors affecting lipase digestibility of emulsified lipids using an in vitro digestion model: Proposal for a standardised pH-stat method. Food Chemistry, 126(2), 498505. 
Lian, G., Malone, M. E., Homan, J. E., \& Norton, I. T. (2004). A mathematical model of volatile release in mouth from the dispersion of gelled emulsion particles. Journal of Controlled Release, 98(1), 139155.

Lundin, L., Golding, M., \& Wooster, T. J. (2008). Understanding food structure and function in developing food for appetite control. Nutrition and Dietetics, 65(Suppl. 3), S79-85.

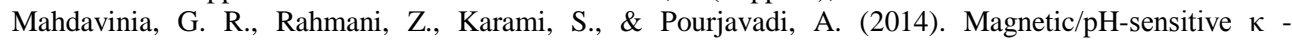
carrageenan/sodium alginate hydrogel nanocomposite beads: preparation, swelling behavior, and drug delivery. Journal of Biomaterials Science, Polymer Edition, 25(17), 1891-1906.

Maljaars, P. W. J., Peters, H. P. F., Kodde, A., Geraedts, M., Troost, F. J., Haddeman, E., \& Masclee, A. A. M. (2011). Length and site of the small intestine exposed to fat influences hunger and food intake. The British Journal of Nutrition, 106(10), 1609-15.

Malone, M. E., \& Appelqvist, I. a M. (2003). Gelled emulsion particles for the controlled release of lipophilic volatiles during eating. Journal of Controlled Release, 90(2), 227-241.

Minekus, M., Alminger, M., Alvito, P., Ballance, S., Bohn, T., Bourlieu, C., ... Brodkorb, A. (2014). A standardised static in vitro digestion method suitable for food - an international consensus. Food \& Function, 5, 1113-1124.

Mun, S., Decker, E. A., Park, Y., Weiss, J., \& McClements, D. J. (2006). Influence of Interfacial Composition on in vitro Digestibility of Emulsified Lipids: Potential Mechanism for Chitosan's Ability to Inhibit Fat Digestion. Food Biophysics, 1(1), 21-29.

Murata, Y., Sasaki, N., Miyamoto, E., \& Kawashima, S. (2000). Use of floating alginate gel beads for stomach-specific drug delivery. European Journal of Pharmaceutics and Biopharmaceutics, 50(2), 221-226.

Norton, I. T., Frith, W. J., \& Ablett, S. (2006). Fluid gels, mixed fluid gels and satiety. Food Hydrocolloids, 20(2-3 SPEC. ISS.), 229-239.

Pignol, D., Ayvazian, L., Kerfelec, B., Timmins, P., Crenon, I., Hermoso, J., ... Chapus, C. (2000). Critical role of micelles in pancreatic lipase activation revealed by small angle neutron scattering. The Journal of Biological Chemistry, 275(6), 4220-4224.

Rayment, P., Wright, P., Hoad, C., Ciampi, E., Haydock, D., Gowland, P., \& Butler, M. F. (2009). Investigation of alginate beads for gastro-intestinal functionality, Part 1: In vitro characterisation. Food Hydrocolloids, 23(3), 816-822.

Rolland, L., Santanach-Carreras, E., Delmas, T., Bibette, J., \& Bremond, N. (2014). Physicochemical properties of aqueous core hydrogel capsules. Soft Matter, 10(48), 9668-74.

Romo, S., \& Perez-Martinez, C. (1997). The use of immobilization in alginate beads for long-term storage of Pseudanabaena galeata (Cyanobacteria) in the laboratory. Journal of Phycology, 33, 1073-1076.

Sarkar, A., Juan, J.-M., Kolodziejczyk, E., Acquistapace, S., Donato-Capel, L., \& Wooster, T. J. (2015). Impact of protein gel porosity on the digestion of lipid emulsions. Journal of Agricultural and Food Chemistry, 63(40), 8829-8837.

Sarkar, A., Murray, B., Holmes, M., Ettelaie, R., Abdalla, A., \& Yang, X. (2016). In vitro digestion of Pickering emulsions stabilized by soft whey protein microgel particles: influence of thermal treatment. Soft Matter, 12(15), 3558-69.

Song, Y., Hahn, H. H., \& Hoffmann, E. (2002). The effects of $\mathrm{pH}$ and $\mathrm{Ca} / \mathrm{P}$ ratio on the precipitation of calcium phosphate. In Chemical water and Wastewater Treatment VII (pp. 349-358).

Stewart, M. B., Gray, S. R., Vasiljevic, T., \& Orbell, J. D. (2014). Exploring the molecular basis for the metalmediated assembly of alginate gels. Carbohydrate Polymers, 102, 246-253.

Tzoumaki, M. V, Moschakis, T., Scholten, E., \& Biliaderis, C. G. (2013). In vitro lipid digestion of chitin nanocrystal stabilized o/w emulsions. Food \& Function, 4(1), 121-9.

van Avesaat, M., Troost, F. J., Ripken, D., Hendriks, H. F., \& Masclee, A. A. M. (2015). Ileal brake activation: macronutrient specific effects on eating behavior? International Journal of Obesity, 39(April), 235243.

Velings, N. M., \& Mestdagh, M. M. (1995). Physico-chemical properties of alginate gel beads. Polymer Gels and Networks, 3(3), 311-330.

Walstra, P. (2003). Physical Chemistry of Foods.

Welch, I., Saunders, K., \& Read, N. W. (1985). Effect of ileal and intravenous infusions of fat emulsions on feeding and satiety in human volunteers. Gastroenterology, 89(6), 1293-1307.

Welch, I., Sepple, C. P., \& Read, N. W. (1988). Comparisons of the effects on satiety and eating behaviour of infusion of lipid into the different regions of the small intestine. Gut, 29, 306-311.

Whitehead, L., Fell, J. T., Collett, J. H., Sharma, H. L., \& Smith, A. M. (1998). Floating dosage forms: An in vivo study demonstrating prolonged gastric retention. Journal of Controlled Release, 55(1), 3-12. 
Workamp, M., Alaie, S., \& Dijksman, J. A. (2016). Coaxial air flow device for the production of millimetersized spherical hydrogel particles. Review of Scientific Instruments, 87(12).

Wright, P. J., Ciampi, E., Hoad, C. L., Weaver, A. C., van Ginkel, M., Marciani, L., ... Rayment, P. (2009). Investigation of alginate gel inhomogeneity in simulated gastro-intestinal conditions using magnetic resonance imaging and transmission electron microscopy. Carbohydrate Polymers, 77(2), 306-315.

Wulff-Pérez, M., Vicente, J. De, Martín-Rodríguez, A., \& Gálvez-Ruiz, M. J. (2012). Controlling lipolysis through steric surfactants: New insights on the controlled degradation of submicron emulsions after oral and intravenous administration. International Journal of Pharmaceutics, 423(2), 161-166.

Zeeb, B., Lopez-Pena, C. L., Weiss, J., \& McClements, D. J. (2015). Controlling lipid digestion using enzyme-induced crosslinking of biopolymer interfacial layers in multilayer emulsions. Food Hydrocolloids, 46, 125-133.

Zeeb, B., Saberi, A. H., Weiss, J., \& McClements, D. J. (2015). Retention and release of oil-in-water emulsions from filled hydrogel beads composed of calcium alginate: impact of emulsifier type and $\mathrm{pH}$. Soft Matter, 11, 2228-2236.

Zhang, Z., Zhang, R., \& McClements, D. J. (2016). Encapsulation of b-carotene in alginate-based hydrogel beads: Impact on physicochemical stability and bioaccessibility. Food Hydrocolloids, 61, 1-10.

Zhang, Z., Zhang, R., Zou, L., Chen, L., Ahmed, Y., Bishri, W. Al, ... McClements, D. J. (2016). Encapsulation of curcumin in polysaccharide-based hydrogel beads : Impact of bead type on lipid digestion and curcumin bioaccessibility. Food Hydrocolloids, 58, 160-170.

Zhang, Z., Zhang, R., Zou, L., \& McClements, D. J. (2016). Tailoring lipid digestion profiles using combined delivery systems: mixtures of nanoemulsions and filled hydrogel beads. RSC Adv., 6(70), 6563165637. 


\section{Chapter 6}

Emulsion-alginate beads:

dynamic in vitro digestion

This chapter is submitted as Corstens, M.N., Berton-Carabin, C.C., Schroën, K., Viau, M., Meynier, A. Emulsion encapsulation in calcium-alginate beads retards lipolysis during dynamic in vitro digestion. 


\section{ABSTRACT}

We assessed the behaviour of encapsulated oil-in-water emulsions in alginate beads under dynamic digestion conditions to test their suitability for ileal brake activation. A dynamic gastrointestinal digestion system (DIDGI) was used with three consecutive compartments simulating the stomach, duodenum and distal small intestine. Digestive media were collected periodically to follow lipolysis, as well as the solubilisation of absorbable lipid species in mixed micelles. Free emulsions (i.e., non-encapsulated) in absence or presence of empty alginate beads were used as controls.

In the free emulsions lipolysis occurred rapidly with micellar solubilisation not rate limiting, which indicates proximal digestion and absorption. Encapsulation of emulsions in calciumalginate beads retarded lipolysis typically for $3 \mathrm{~h}$ by diffusion limitation. Our findings do not only increase the understanding of the dynamics of lipid digestion, but also directly link to the design of encapsulates (size and mesh size) for long-term weight management strategies. 


\subsection{INTRODUCTION}

Controlling lipid digestion is of growing interest for many reasons. On the one hand, enhancing the gastrointestinal (GI) delivery and bioavailability of health-promoting lipids (e.g., long chain polyunsaturated fatty acids) is desired; on the other hand, delaying lipid digestion can be of interest, to allow undigested lipids to reach distal parts of the GI tract where they activate an intestinal brake mechanism. The ileal brake is a negative feedback mechanism that originates from the ileum, and that targets the proximal GI tract including stomach, gallbladder and pancreas, and also the central nervous system (Alleleyn, van Avesaat, Troost, \& Masclee, 2016; Cummings \& Overduin, 2007; Maljaars, Peters, Mela, \& Masclee, 2008; Van Citters \& Lin, 2006). These feedback processes impair food digestion, appetite sensations and food intake, and are able to increase feelings of satiety and satiation (Maljaars et al., 2008; van Avesaat, Troost, Ripken, Hendriks, \& Masclee, 2015). To the best of our knowledge, the activation of the ileal brake has only been achieved after direct infusion of nutrients in targeted parts of the human intestine via a catheter. In this paper, we focus on a food-based approach that controls lipolysis of encapsulated lipid, which could then activate the ileal brake, and thus be part of a non-invasive treatment for long-term weight management.

Lipolysis is a reaction controlled by the oil-water interface accessibility of lipase, which in turn is controlled by physicochemical characteristics of the surface of oil droplets, such as interfacial area, composition and structure (Armand, 2007). Interfacial area is determined by the emulsion droplet size, and composition is related to the nature of the components present at the interface, which determines the stability in the GI tract (Golding et al., 2011; Lundin, Golding, \& Wooster, 2008). It has been postulated that the extent of lipolysis depends on the structure that these components form at the interface, via its resistance against adsorption of bile salts and lipase (Corstens, Berton-Carabin, de Vries, et al., 2017). Different designs of emulsion interfaces have been proposed to control and delay lipolysis: synthetic surfactants that provide steric hindrance (Chu et al., 2009; Wulff-Pérez, Vicente, Martín-Rodríguez, \& Gálvez-Ruiz, 2012), thick interfacial films through layer-by-layer adsorption of biopolymers (Corstens, Berton-Carabin, Kester, et al., 2017; Hu, Li, Decker, Xiao, \& McClements, 2011; Klinkesorn \& McClements, 2010; Mun, Decker, Park, Weiss, \& McClements, 2006; Zeeb, Lopez-Pena, Weiss, \& McClements, 2015), and particle-based layers (Sarkar et al., 2016; Tzoumaki, Moschakis, Scholten, \& Biliaderis, 2013). General trends indicate that these approaches seem insufficient to induce the ileal brake as most of the designed interfaces appeared unstable under gastric conditions and consequently lipid droplets emptied in the duodenum rather unprotected and then accessible to lipase.

An alternative approach is to control the diffusion of lipase toward its substrate, by trapping lipid droplets in a hydrogel matrix. The latter can be either digestible, such as proteins 
(Dekkers, Kolodziejczyk, Acquistapace, Engmann, \& Wooster, 2016; Sarkar et al., 2015), or indigestible, such as alginate (Corstens, Berton-Carabin, Elichiry-Ortiz, et al., 2017; Li, Hu, Du, Xiao, \& McClements, 2011; Zhang et al., 2016). Proteolysis of protein-based hydrogels leads to surface erosion (Sarkar et al., 2015), whereas indigestible hydrogels remain intact and hence can limit lipase diffusion, and in that way control lipolysis. This process can be fine-tuned by variation in gel bead size and pore size, as recently shown for a static in vitro model using oil-in-water $(\mathrm{O} / \mathrm{W})$ emulsions $\left(d_{32} \sim 25 \mu \mathrm{m}\right)$ encapsulated in calcium-alginate hydrogel beads (bead diameter 0.5-1.7 mm; pore size 5-10 nm) (Corstens, Berton-Carabin, Elichiry-Ortiz, et al., 2017). These encouraging results were obtained under static in vitro conditions; therefore, we further test the behaviour of these beads under dynamic in vitro conditions that are closer to in vivo physiological conditions.

Several in vitro equipment are available to take into account part of the dynamic aspect of human digestion (Guerra et al., 2012; Oosterveld, Minekus, Bomhof, Zoet, \& van Aken, 2016; Verhoeckx et al., 2015). The most used dynamic in vitro model of the upper GI tract is the TNO gastrointestinal model 1 (TIM-1), which contains four compartments: stomach, duodenum, jejunum, and ileum. It is able to control the GI transit, regulate $\mathrm{pH}$, secretion, and absorption from the small intestine (Minekus, Marteau, Havenaar, \& Huis in 't Veld, 1995). The French National Institute for Agricultural Research (INRA) has developed an alternative model of the upper GI tract that is simpler, cheaper and easier: the dynamic gastrointestinal dynamic digestion system (DIDGI) (Ménard et al., 2014; Verhoeckx et al., 2015). The DIDGI system has been validated with in vivo data for protein digestion using only two compartments: stomach and small intestine that are computer-controlled in regard to transit times, pH kinetics, and secretions (Ménard et al., 2014). For the current study, a third compartment was added to mimic the second part of the small intestine (jejunum + ileum).

Our objective was to assess the behaviour of $\mathrm{O} / \mathrm{W}$ emulsions, either free or encapsulated in hydrogel beads, under dynamic digestion conditions to test their potentiality to induce the ileal brake. For encapsulation, a calcium-alginate hydrogel matrix was used as described previously (Corstens, Berton-Carabin, Elichiry-Ortiz, et al., 2017). Both free and encapsulated emulsions were mixed with a fat-free yogurt that mimics a meal and then introduced in a dynamic three-compartment model of the upper GI tract (DIDGI). The potential effect of the encapsulation material itself on lipolysis was tested with a control meal made of fat-free yogurt mixed with free emulsion and empty calcium-alginate beads. Aliquots of digestive media were sampled over time and analysed for both the total extent of lipolysis and the bioaccessible fraction (i.e., solubilized in mixed micelles), which allowed us to assess the potential of our encapsulate to delay lipolysis. 


\subsection{MATERIAL AND METHODS}

\subsubsection{Materials}

Safflower oil was purchased from De Wit Specialty oils (19200 Safflower Oil High Linoleic Refined, the Netherlands), and consists mostly of linoleic acid, as shown in Supplementary Table 6.3. Fat-free yogurt (composition per $100 \mathrm{~g}$, as given by the manufacturer: $0 \mathrm{~g}$ lipids, 4.0 g carbohydrates, 4.7 g proteins, $135 \mathrm{mg}$ calcium) was purchased at a local supermarket, and produced by Campina (the Netherlands; $\mathrm{pH}$ around 4.4). Whey protein isolate (WPI) was obtained from Davisco Foods International (BiPro, Eden Prairie, Minnesota, USA; purity 97.5\%). From Sigma Aldrich (St. Louis, MO, USA, and Saint-Quentin Fallavier, France) we purchased sodium alginate, sodium bicarbonate, sodium chloride, calcium chloride, sodium phosphate dibasic, sodium phosphate monobasic, hydrochloric acid, potassium chloride, heptadecanoic acid (GC standard), porcine gastric mucosa (3,200-4,500 U.mg-1 protein), Amano Lipase A from Aspergillus niger $\left(120 \mathrm{U} \cdot \mathrm{mg}^{-1}\right.$ at $\left.\mathrm{pH} 6.5,45^{\circ} \mathrm{C}\right)$, pancreatin from porcine pancreas $(8 \times$ USP specification; including trypsin, amylase, lipase, ribonuclease, protease), lipase from porcine pancreas (Type II, 100-500 U· $\mathrm{mg}^{-1}$ protein on olive oil), porcine bile extract (crude extract, purity estimated to be 30 to $60 \%$ containing glycine and taurine conjugates of hyodeoxycholic acid and other bile salts according to the supplier). From Carlo Erba Reagents (Val de Reuil, France) we purchased ammonia solution (30\%), cyclohexane, and propan-2-ol; and from Biosolve (Valkenswaard, the Netherlands) methanol absolute (HPLC supra-gradient), n-hexane (HPLC), chloroform (ethanol-stabilized, HPLC). All materials were used directly without further purification. Millipore ultrapure water (18.2 $\mathrm{M} \Omega$ ) was used throughout the study.

\subsubsection{Methods}

\section{Meal preparation}

Emulsion preparation. Safflower oil was mixed with WPI solution (1 wt\% in $10 \mathrm{mM}$ phosphate buffer $\mathrm{pH}$ 7.0) in a ratio of 1:4 (w/w), using a rotor-stator homogenizer (Silent Crusher, Heidolph basic Ultra-Turrax homogenizer, Germany) for 5 min at 13·10 $\mathrm{rpm}, 2 \mathrm{~min}$ rest, and $2 \mathrm{~min}$ at $15 \cdot 10^{3} \mathrm{rpm}$ to reach an average droplet size of $\sim 25 \mu \mathrm{m}$. Emulsions were used within $2 \mathrm{~h}$ either to prepare a meal or to encapsulate in calcium-alginate beads.

Bead preparation. The alginate solution (10 mM phosphate buffer $\mathrm{pH} 7.0)$ was hydrated overnight at $4{ }^{\circ} \mathrm{C}$, and then mixed with the emulsion (magnetic stirrer). The final composition of the mixture was $10 \mathrm{wt} \%$ lipid and $3 \mathrm{wt} \%$ alginate in the continuous phase. This mixture was injected in a $0.45 \mathrm{M} \mathrm{CaCl}_{2}$ bath to form the gel beads as described previously (Corstens, Berton-Carabin, Elichiry-Ortiz, et al., 2017). The formed emulsion-alginate beads were 
stored overnight at $4{ }^{\circ} \mathrm{C}$ to allow hardening, and were washed and filtered before further use. Empty beads were produced with $1 \%$ alginate solution using the same method.

Meal preparation. The maximum meal volume that can be introduced into the DIDGI in a single dose through a syringe pump was $120 \mathrm{~mL}$. Fat-free yogurt (37.5 g) was enriched with $2.5 \mathrm{~g}$ emulsified safflower oil (2.1\% lipid in the meals) either encapsulated as emulsionalginate beads (total $25 \mathrm{~g}$ beads, $10 \mathrm{wt} \%$ lipid), or as a free emulsion (12.5 g emulsion, 20 wt\% lipid) with or without empty calcium-alginate beads ( $22.5 \mathrm{~g}$ beads, $0 \mathrm{wt} \%$ lipid). Water was added to obtain a similar total volume for all meals. The composition of the three tested meals is summarized in Table 6.1. The meals were stirred for homogeneity ( 1 h, 300 rpm, $4{ }^{\circ} \mathrm{C}$ ) before introduction into the dynamic in vitro digestion system. The caloric content of the meals ranged from 37 to $40 \mathrm{kcal}$ : fat-free yogurt with free emulsion $37 \mathrm{kcal}$ (free emulsion meal), with free emulsion and empty beads $38 \mathrm{kcal}$ (free emulsion meal with empty beads), and with emulsion-alginate beads $40 \mathrm{kcal}$ (encapsulated emulsion meal).

Table 6.1. Composition of the meals.

\begin{tabular}{lccc}
\hline \multicolumn{1}{c}{ (gram) } & Free emulsion & $\begin{array}{c}\text { Free emulsion } \\
\text { with empty beads }\end{array}$ & $\begin{array}{c}\text { Encapsulated } \\
\text { emulsion }\end{array}$ \\
\hline Fat-free yogurt & 37.5 & 37.5 & 37.5 \\
Emulsion-alginate beads & - & - & 25 \\
Empty alginate beads & - & 22.5 & - \\
Emulsion (20\% oil) & 12.5 & 12.5 & - \\
Water & 80 & 57.5 & 67.5 \\
\hline
\end{tabular}

Sample characterization

Particle size distribution. The particle size distribution of both emulsions and beads was determined using a laser diffraction particle size analyser (LA-960, Horiba Scientific, Jobin Yvon, France) and the Mie scattering model. Samples were dispersed in ultrapure water in the stirred dispersion unit, until an obscuration around $7-10 \%$ was obtained for emulsions and around $2-4 \%$ for beads. The refractive index (RI) of the dispersant was that of water (1.333), and the RI of the dispersed phase that of oil (1.470). Light microscopy images were used to get an impression of the bead size.

Lipid content. The lipids were extracted based on partitioning of lipids in different solvents, using an isopropanol/hexane medium (Hara \& Radin, 1978). Total lipids were extracted from the meal and digestive media $(1.5 \mathrm{~mL}$ of the meal, gastric media, and duodenal media; and 3 $\mathrm{mL}$ of the distal media) by adding 10 volumes of hexane/isopropanol (3:2 (v/v)) and 0.4 volume of $150 \mathrm{mM} \mathrm{NaCl}$ per volume of digestive media. The samples were vortexed ( $1 \mathrm{~min}$, $3 \cdot 10^{3} \mathrm{rpm}$ ) and then centrifuged for $5 \mathrm{~min}$ at $2 \cdot 10^{3} \times \mathrm{g}$. The upper hexane phase was collected, and replaced with an equivalent volume of fresh hexane. This procedure was repeated twice 
to ensure total extraction and recovery of lipids. The three hexane phases were combined; solvent was evaporated under reduced pressure (Rotavapor, Büchi, France), and dried completely under a stream of nitrogen to quantify the lipid content by weighing.

\section{Dynamic in vitro model of the gastrointestinal tract}

Experimental device. The gastrointestinal digestion system (DIDGI) was described previously as a two-compartment setup (Ménard et al., 2014), but here consists of three consecutive compartments simulating the stomach, duodenum, and distal small intestine, respectively. In short, each compartment was equipped with $\mathrm{pH}$ sensor (Electrode InPro 4801i/SG/120, reference 52003581, Metler Toledo, France), temperature sensor, and a double-walled jacket for temperature control $\left(37^{\circ} \mathrm{C}\right)$. Besides, DIDGI is equipped with several peristaltic pumps (Verder, France) to control respectively the flow of meal, $\mathrm{HCl}$, $\mathrm{NaHCO}_{3}$, bile, enzymes, and the emptying of each compartment. The parameters of the dynamic digestion (Table 6.2) were carefully selected based on literature data collected either from in vivo (Berrada, Lemeland, Laroche, Thouvenot, \& Piaia, 1991; Calbet \& MacLean, 1997; Malagelada, Go, \& Summerskill, 1979) or from in vitro data (Blanquet et al., 2004; Guerra et al., 2012; Minekus, Marteau, Havenaar, \& Huis in 't Veld, 1995; Villemejane et al., 2016). The system was controlled by a software for stomach regulation and monitoring (StoRM (Guillemin, Perret, Ménard, \& Picque, 2010)).

Computer-controlled transit. The digestion started when a computer-controlled pump introduced $120 \mathrm{~mL}$ of the meal into the gastric compartment within three minutes (40 $\left.\mathrm{mL} \cdot \mathrm{min}^{-1}\right)$. To control the transit time of the chyme in each compartment, a power exponential mathematical equation for gastric and intestinal delivery was used (Equation 6.1):

$$
F=2^{-\left(t / t_{1 / 2}\right)^{\beta}}
$$

Equation 6.1

where $F$ represents the fraction of the chyme remaining in the dedicated compartment, $t$ the time of delivery, $t_{1 / 2}$ the half time of delivery and $\beta$ the coefficient describing the shape of the curve (Elashoff, Reedy, \& Meyer, 1982). To determine these parameters for the studied meals, the parameters of Minekus and co-workers (Minekus et al., 1995) were considered and adapted for meal consistency by fitting the data of fermented milk (Berrada et al., 1991) to Equation 6.1, and fixed at $t \frac{1}{2}=45 \mathrm{~min}$ and $\beta=1.5$ for the gastric compartment. The gastric compartment was emptied into the duodenal compartment through a sieve with $2 \mathrm{~mm}$ holes mimicking the sieving effect of the pylorus in humans (Kong \& Singh, 2008). The volume in the three compartments over the timescale of an experiment can be found in Supplementary Figure 6.7. 
$\mathrm{pH}$ control. After introduction of the meal, the $\mathrm{pH}$ of the gastric compartment was equal to that of the meal (close to 4.4) and followed a linear acidification curve with a slope of - 0.02 $\mathrm{min}^{-1}$ to decrease down to $\mathrm{pH} 2.0$ in 120 minutes, in accordance with previous studies (Dekkers et al., 2016; Villemejane et al., 2016). Duodenal pH was kept constant at 6.5. The $\mathrm{pH}$ was measured continuously and regulated based on a feedback loop through secretion of $\mathrm{HCl}(1 \mathrm{M})$ into the gastric compartment, or $\mathrm{NaHCO}_{3}(1 \mathrm{M})$ into the duodenal compartment. These concentrations of $\mathrm{HCl}$ and $\mathrm{NaHCO}_{3}$ were selected experimentally to control the $\mathrm{pH}$ and limit volume increase, without causing aggregation problems with the proteins in the yogurt.

Table 6.2. Parameters of dynamic in vitro gastrointestinal digestion.

\begin{tabular}{|c|c|c|}
\hline \multicolumn{3}{|l|}{ Gastric compartment } \\
\hline Ingestion & $120 \mathrm{~mL}$ & $40 \mathrm{~mL} / \mathrm{min}$ \\
\hline Fasted volume & $\begin{array}{l}30 \mathrm{~mL} \\
\text { (saline solution } \mathrm{pH} 2.5 \text { ) }\end{array}$ & \\
\hline $\mathrm{pH}$ acidification curve & $\mathrm{pH}=-0.021 \cdot \mathrm{t}+\mathrm{pH}$ meal & \\
\hline \multirow[t]{2}{*}{ Secretion } & Pepsin $(8.2$ g/L) & $0.4 \mathrm{~mL} / \mathrm{min}$ \\
\hline & Gastric lipase (3.3 g/L) & $0.25 \mathrm{~mL} / \mathrm{min}$ \\
\hline Agitation & $100 \mathrm{rpm}$ & \\
\hline \multirow[t]{2}{*}{ Transit (Equation 6.1) } & $t_{1 / 2}$ & $45 \mathrm{~min}$ \\
\hline & $\beta$ & 1.5 \\
\hline Sampling time & $\mathrm{t}=45,90 \mathrm{~min}$ & \\
\hline \multicolumn{3}{|c|}{ Duodenal compartment } \\
\hline Fasted volume & $\begin{array}{l}\sim 10 \mathrm{~mL} \\
\text { bile and enzyme solution }\end{array}$ & \\
\hline $\mathrm{pH}$ & Maintained at 6.5 & \\
\hline Secretion & $\begin{array}{l}\text { Bile ( } 55 \mathrm{~g} / \mathrm{L} \text { bile extract) } \\
\text { Intestinal enzymes } \\
(4 \mathrm{~g} / \mathrm{L} \text { pancreatin }+ \\
2 \mathrm{~g} / \mathrm{L} \text { lipase) }\end{array}$ & $\begin{array}{l}0.4 \mathrm{~mL} / \mathrm{min} \\
0.25 \mathrm{~mL} / \mathrm{min}\end{array}$ \\
\hline Agitation & $50 \mathrm{rpm}$ & \\
\hline \multirow[t]{2}{*}{ Transit } & $t_{1 / 2}$ & $60 \min$ \\
\hline & $\beta$ & 1 \\
\hline Sampling time & $\mathrm{t}=30,60,90,120 \mathrm{~min}$ & \\
\hline \multicolumn{3}{|l|}{ Distal compartment } \\
\hline $\mathrm{pH}$ & $7.0-7.3$ & monitored, not adjusted \\
\hline Agitation & $50 \mathrm{rpm}$ & \\
\hline Sampling time & $\mathrm{t}=60,90,120,180,240 \mathrm{~min}$ & \\
\hline
\end{tabular}


Gastrointestinal conditions. The amount and origin of 'gastric' lipase varies greatly between studies (Sams, Paume, Giallo, \& Carrière, 2016). Due to the lack of commercially available human gastric lipase, we decided to include a microbial lipase (from Aspergillus niger) in the gastric phase of the dynamic digestion. The concentration and secretion rate have been chosen after preliminary explorative static digestion experiments, including various relevant $\mathrm{pH}$ values. Based on a combination of gastric $\mathrm{pH}$ profile, lipase activity at these $\mathrm{pH}$ values, and emptying time, we optimized secretion of lipase during the gastric digestion to have a physiologically relevant gastric lipolysis (final release of free fatty acid around 5-10\%) in free emulsion meals (data on $\mathrm{pH}$ dependence of gastric lipase not shown). The software controlled the peristaltic pumps that injected pepsin $\left(8.2 \mathrm{~g} \cdot \mathrm{L}^{-1} ; 0.4 \mathrm{~mL} \cdot \mathrm{min}^{-1}\right)$ and gastric lipase (3.3 g. $\left.\mathrm{L}^{-1} ; 0.25 \mathrm{~mL} \cdot \mathrm{min}^{-1}\right)$ into the gastric compartment, and bile $\left(55 \mathrm{~g} \cdot \mathrm{L}^{-1} ; 0.4\right.$ $\left.\mathrm{mL} \cdot \mathrm{min}^{-1}\right)$ and a pancreatic lipase mixture $\left(4 \mathrm{~g} \cdot \mathrm{L}^{-1}\right.$ pancreatin, $2 \mathrm{~g} \cdot \mathrm{L}^{-1}$ lipase; $\left.0.25 \mathrm{~mL} \cdot \mathrm{min}^{-1}\right)$ into the duodenal compartment. Digestive enzymes and bile were rehydrated in saline solution (120 mM NaCl, $5 \mathrm{mM} \mathrm{KCl,} 6 \mathrm{mM} \mathrm{CaCl}_{2}$ ) and kept on ice throughout the experiment to avoid autolysis. To check the various delivered volumes, all fluid-containers were weighed before and after digestion.

During each of the three independent digestions per meal, aliquots of digestive media were sampled from the compartments over time, named digestive media in the rest of the text, to assess the total extent of lipolysis as well as the solubilisation of absorbable lipid species in mixed micelles, as both described next.

\section{Assessment of lipolysis}

To determine lipolysis extent, digestive media was collected directly into tubes containing the extraction solvent (hexane/isopropanol) that inactivated enzymes. This was done for the meal (1.5 mL; $\mathrm{t}=0 \mathrm{~min})$, gastric media $(1.5 \mathrm{~mL} ; \mathrm{t}=45,90 \mathrm{~min})$, duodenal media (1.5 mL; $\mathrm{t}=30,60,90,120 \mathrm{~min})$, and the distal compartment (3 mL; $\mathrm{t}=60,90,120,180,240 \mathrm{~min})$. Then, total lipids were extracted as described previously, and HPLC analysis was performed to separate and quantify the different lipid components: free fatty acids (FFA), monoacylglycerols (MAG), diacylglycerols (DAG), and triacylglycerols (TAG).

HPLC analysis. The total lipid extract was dissolved in chloroform and diluted to a final concentration below $0.3 \mathrm{mg}$ lipid per $\mathrm{mL}$ for HPLC injection. Normal phase separation as well as identification and quantification were described previously (Kenmogne-Domguia, H.B., Meynier, A., Viau, M., Llamas, G., Genot, 2012). From a quadratic fit between the peak area and the injected amount, the weight percentage of each lipid class was calculated using the calibration curve, and afterwards converted into molar concentrations.

Extent of lipolysis. For each sampling time, the quantitative lipid profile from HPLC was used to calculate the extent of lipolysis. Molar fractions were calculated using mean 
molecular weights for FFA of $276 \mathrm{~g} \cdot \mathrm{mol}^{-1}$, MAG of $350 \mathrm{~g} \cdot \mathrm{mol}^{-1}$, DAG of $608 \mathrm{~g} \cdot \mathrm{mol}^{-1}$, and TAG of $866 \mathrm{~g} \cdot \mathrm{mol}^{-1}$, based on the fatty acid composition of safflower oil (Supplementary Table 6.3) and a blank digestion (10 mM buffer, $\mathrm{pH} 4.4$ ) to correct for the endogenous lipid contributions of bile and enzyme extracts. The lipolysis degree (LD) was calculated as the percentage of FFA (mol) versus the total acyl chains (mol) present per lipid class (Couëdelo et al., 2015; de Oliveira et al., 2016) as indicated in following equation (Equation 6.2):

$$
L D=100 \% \times \frac{[F F A]}{3 \times[T A G]+2 \times[D A G]+[M A G]+[F F A]} \quad \text { Equation } 6.3
$$

where [FFA], [MAG], [DAG], and [TAG] are the concentrations in the digestive media $\left(\mu \mathrm{mol} \cdot \mathrm{mL}^{-1}\right)$ after blank correction, and LD the lipolysis degree $\left(\% \mathrm{~mol} \cdot \mathrm{mol} \mathrm{total} \mathrm{l}^{-1}\right)$. In general, it is expected that the lipases that are used hydrolyse the fatty acids at the sn1 and sn3 positions, leading to a maximum lipolysis degree of $67 \%$.

\section{Assessment of bioaccessibility}

In order to determine absorbable lipids, namely those solubilized in mixed micelles, the fatty acid (FA) composition of the micellar phase was compared to that of the lipid extracted from the total media.

\section{FA analysis in the lipids extracted from total media}

The total amount of FAs present in the digestive media over time was determined for the total lipid extracts (TL) as FA methyl esters (FAMEs) according to (Morrison \& Smith, 1964) as described previously (Meynier et al., 2014). As mentioned, these values were corrected for the endogenous lipid contributions of the digestive media, especially from bile as revealed by blank digestion.

\section{FA analysis in the micellar phase}

The so-called micellar phase containing lipolytic products (i.e., FFA and MAG) was separated from digestive media by centrifugation. For that, $1.5 \mathrm{~mL}$ of digestive media was transferred to tubes and centrifuged during $45 \mathrm{~min}$ at $21 \cdot 10^{3} \times \mathrm{g}$ (Micro Ultracentrifuge Hettich Universal 320R, Germany). The aqueous fraction was collected using an 18-gauge needle fitted to a $1 \mathrm{~mL}$ syringe and then filtered through a cellulose acetate membrane (pore size $0.22 \mu \mathrm{m}$; Sartorius Company, Germany). An aliquot of the filtrate, typically $500 \mu \mathrm{L}$, was transferred to a glass tube for direct methylation without prior extraction. Then, $100 \mu \mathrm{L}$ internal standard (heptadecanoic acid: $1 \mathrm{mg} \cdot \mathrm{mL}^{-1}$ in acetone/methanol $(2: 1(\mathrm{v} / \mathrm{v})), 2 \mathrm{~mL}$ methanol and $400 \mu \mathrm{L}$ sulfuric acid was added and the glass tubes were closed. The samples were shaken for $30 \mathrm{~s}$ and heated to $100{ }^{\circ} \mathrm{C}$ for $60 \mathrm{~min}$. After cooling to room temperature, 
$1 \mathrm{~mL}$ of water was added to the solution, and FAMEs were extracted with $2 \mathrm{~mL}$ of cyclohexane. An aliquot of the upper cyclohexane phase $(1 \mu \mathrm{L})$ was analysed for the FAs in the micellar lipid extracts, and the values were corrected with the previously mentioned blank to prevent any effects caused by the digestive liquids.

The amount of linoleic acid (C18:2), the major fatty acid of safflower oil, incorporated in the micellar phase over time can be used as a measure for satiety induction (Maljaars, Romeyn, Haddeman, Peters, \& Masclee, 2009). Bioaccessibility was defined as the ratio between micellar and total amounts (after correction for digestive components).

\subsubsection{Data analysis}

For each meal (free emulsion meal, free emulsion meal with empty beads, encapsulated emulsion meal), we performed three independent digestion experiments. Mean values are reported with standard deviations. We analysed the effect of meal, time, compartment (stomach, duodenum, distal small intestine), and their interactions. The dependent variables were tested separately, and included the lipolysis degree, FA concentrations in micelles and in total, and their relative amounts (percentage FA in micellar phase relative to FA in total lipids; representing bioaccessibility). Analysis of variance (ANOVA) was performed using the statistical software package SPSS Version 23.0.0.2 (IBM@ SPSS Statistics, Chicago, IL, USA). For significant ANOVA outcomes, means were compared pairwise $(p<0.05)$ using post hoc Tukey HSD.

\subsection{RESULTS AND DISCUSSION}

\subsubsection{Lipid distribution in the different compartments}

To evaluate the homogeneity of the digestive media in each studied compartment, the lipid concentration in each sampling point was determined and compared with predicted values calculated by StoRM software, taking into account the various secretions as well as the emptying, and assuming the homogeneity of both content and emptying (Figure 6.1). As expected, the lipid concentration varied with time of digestion and compartment. In the stomach, the measured content was in good agreement with the predicted values, with no differences between the three meals (Figure 6.1a). This result indicates that neither the presence of alginate, nor the encapsulation of the emulsion in alginate beads affects the homogeneity within the gastric compartment, and thus gastric emptying can be considered homogeneous. This finding differs from results obtained with a tiny-TIM system for dynamic digestion of WPI-stabilized emulsions, showing non-homogeneous emptying (Oosterveld et al., 2016). 
In the duodenal compartment (Figure 6.1b), the results differed greatly between the encapsulated emulsion meal and the two other meals that contain free emulsion. The lipid concentrations for both free emulsion meals are lower than predicted during the first hour of the duodenal digestion and then increased drastically until the end of duodenum emptying. The encapsulated emulsion behaves almost as predicted by the software, indicating a rather homogenous lipid distribution. Possibly, the free lipid droplets have flocculated or destabilized at the beginning of the duodenal digestion and consequently layered at the top of the compartment (Golding et al., 2011), where lipolysis can proceed longer since the exit is situated at the bottom of the compartment.

When the meals entered the distal compartment, representing the jejunum and ileum, the initial lipid concentration was slightly lower than calculated for both free emulsion meals due to the non-homogeneous emptying of the duodenal compartment. For the encapsulated emulsion meal, the lipid concentration was close to the predicted values indicative of homogenous content and emptying. More details on the lipolysis in the various compartments are given in the next section.
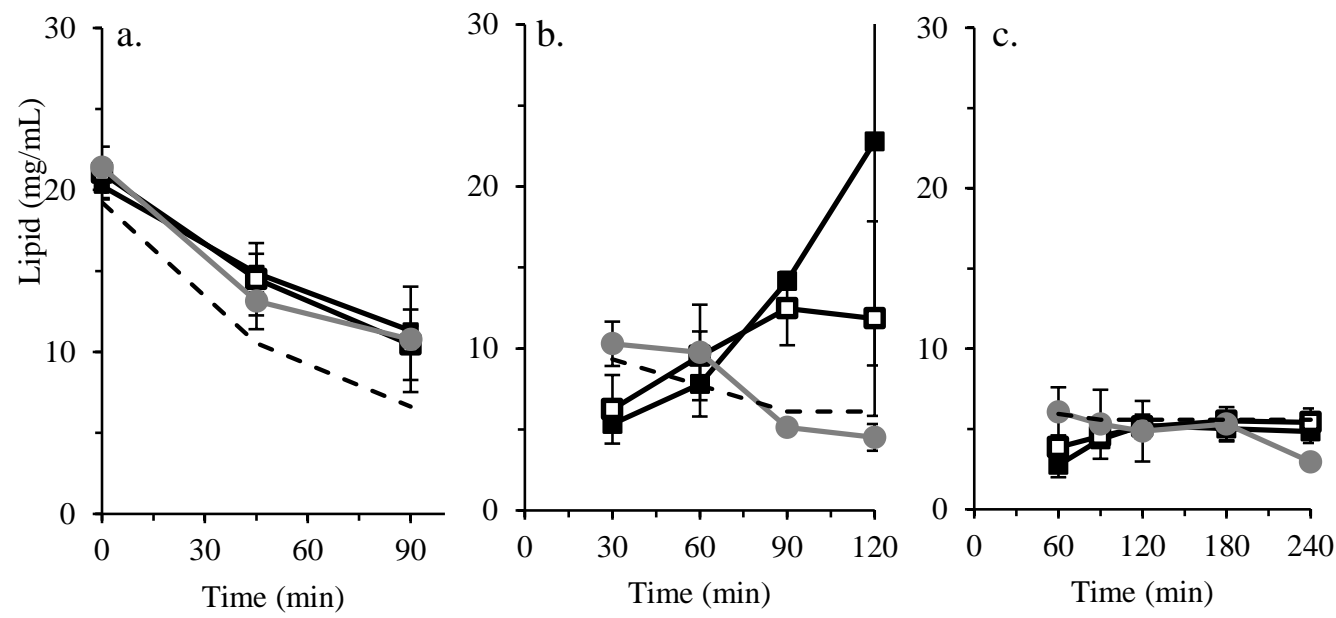

Figure 6.1. Evolution of lipid concentration over time in the a) gastric, b) duodenal, c) distal small intestinal compartment, during dynamic in vitro digestion of free emulsion meal (black square), free emulsion meal with empty beads (empty square), encapsulated emulsion meal (grey circle). Mean values with standard deviations are shown $(n=3)$; the expected values for ideal mixing are indicated by the dashed line. 


\subsubsection{Evolution of lipolysis in the different compartments}

The time course of lipolysis was represented separately in the three consecutive compartments of the DIDGI system as lipolysis degree (LD) in Figure 6.2. ANOVA analysis highlighted that meal type significantly affected the extent of lipolysis per compartment (as measured by LD, $p=0.01$ ), but the interaction time*compartment was not significant $(p=0.15)$. For the statistical analysis, we therefore focus on the meal types per compartment using paired comparison (post hoc Tukey HSD, $p<0.05$ ).

\section{Lipolysis in the gastric compartment}

The gastric lipolysis was limited for all meals and ranged from nearly null after 90 min (encapsulated emulsion meal) to $8.9 \%$ (the free emulsion meal with empty beads). This result confirmed that the lipase concentration and flowrate was representative for in vivo data in healthy adults (Armand, 2007). Figure 6.3 shows the evolution of the relative molar fractions of the different lipid species throughout the digestion. Free emulsion meal with empty beads had a significantly higher LD (Figure 6.2b) compared to the encapsulated emulsion meal (Figure 6.2c), and shows the highest amount of free fatty acids (FFA) and diacylglycerols (DAG) in the end of the gastric phase (Figure 6.3b). Since the lipolysis degree is low, monoacylglycerols (MAG) were not detected in the gastric compartment.

\section{Lipolysis in the small intestinal compartments}

Lipolysis took to a larger extent place in the small intestinal compartments, reaching about half of the maximum lipolysis degree in the duodenal compartment for the free emulsion meal (Figure 6.2b), and being completed (assuming that two FFAs can be released from one triacylglycerol (TAG)) in the distal compartment (Figure 6.2c, LD $63 \pm 6 \%$ after $4 \mathrm{~h}$ incubation). This indicates almost completed lipolysis of emulsified lipids in the jejunum, which is in line with physiological data (Armand, 2007).

Figure 6.2b also shows that lipolysis was far more intense at the first duodenal sampling point (after $30 \mathrm{~min}$ ) for both free emulsion meals (LD of 28-34\%), compared to the emulsion-alginate beads (LD of 6\%). LD increased at the end of the duodenal phase (120 min) for both free emulsion meals, whereas the LD in the encapsulated emulsion meal evolved more gradually. When comparing the duodenal phase pairwise it was clear that entrapment of the emulsion inside the alginate bead reduced lipolysis significantly. As shown in Figure 6.3, the relative proportion of MAGs increased immediately upon entering the duodenal phase for the free emulsion meals, whereas the encapsulated emulsion meal only shows an increase at the very last sampling point. 

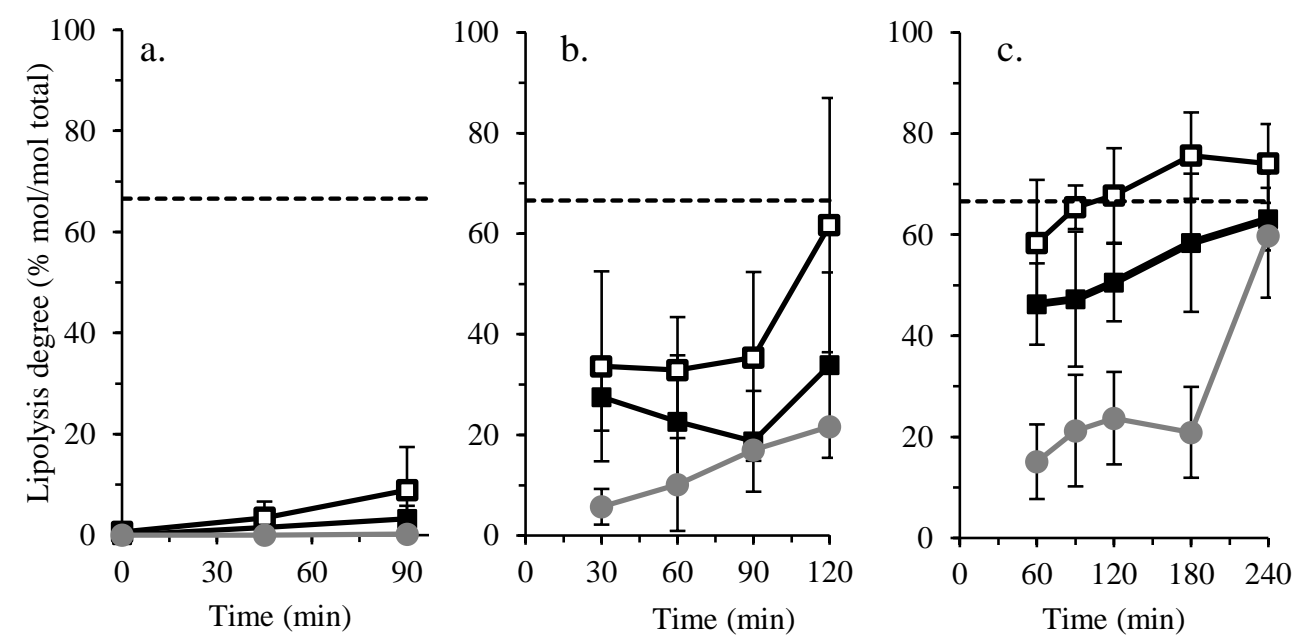

Figure 6.2. Evolution of lipolysis degree (\% mol/mol total) over time in the a) gastric, b) duodenal, and c) distal small intestinal compartment, during in vitro digestion of free emulsion meal (black square), free emulsion meal with empty beads (empty square), encapsulated emulsion meal (grey circle). Mean values with standard deviations are shown $(n=3)$; the dashed line indicates the theoretical maximum value based on the release of two moles fatty acids per mole triacylglycerol.

In the distal small intestinal compartment (Figure 6.2c), a huge difference was noticed in initial LD between free emulsion meals (LD 46-58\%) and the encapsulated emulsion meal (LD 15\%). When comparing the overall LD pairwise between the meals, it was significantly lower for the encapsulated emulsion meal compared to both free emulsion meals. Interestingly, the extent of lipolysis in the encapsulated emulsion meal increased rapidly only after 180 min of intestinal digestion, indicating retarded release from the emulsion-alginate beads, as also detailed in Figure 6.3c.

For the free emulsion meals, lipolysis continues within the distal small intestinal phase as shown in Figure 6.3a, and $\mathbf{b}$ through the decrease of TAG fraction towards zero after 240 min. Conversely, in the encapsulated emulsion meal the relative TAG fraction was still 13\%. Interestingly, in the presence of both types of alginate beads, empty and emulsion-filled, the relative DAG fraction does not go to zero even after $240 \mathrm{~min}$.

In both intestinal compartments, the free emulsion meal with empty beads had a slightly but significantly higher LD than the free emulsion meal without empty beads, indicating that the presence of empty beads may have slightly modified lipase activity, but did not cause lipase inhibition as suggested previously (Wilcox, Brownlee, Richardson, Dettmar, \& Pearson, 2014). The retarded lipolysis with emulsion-alginate beads is caused by reduced lipase diffusion in the calcium-alginate hydrogel matrix (Li et al., 2011). The beads were able to 
retard lipolysis in the first $3 \mathrm{~h}$ of GI digestion, but after $4 \mathrm{~h}$ most of the FFAs were released in the distal small intestinal compartment (Figure 6.3c). It is known that these beads slightly reduce their volume in acidic conditions, protection the core from the gastric environment, while they increase in volume under more neutral physiological conditions in presence of calcium as used here and in a previous study under static in vitro conditions (Corstens, Berton-Carabin, Elichiry-Ortiz, et al., 2017), which would facilitate release. Both aspects are expected to contribute positively in a product that is to be used for weight management.

-FFA $=\mathrm{MAG} \square \mathrm{DAG}=\mathrm{TAG}$

a.

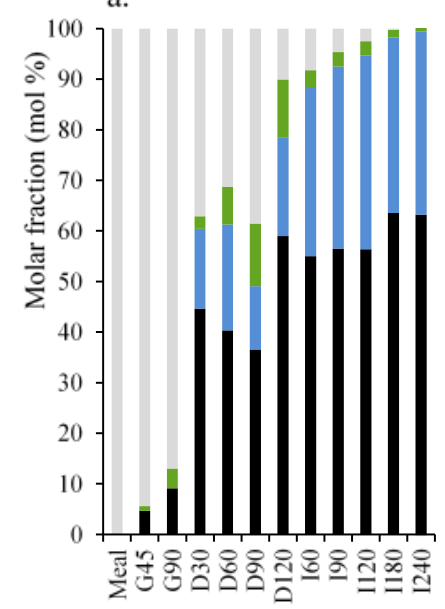

b.

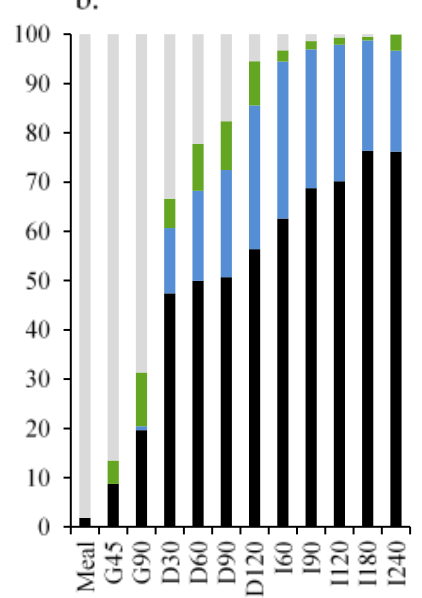

c.

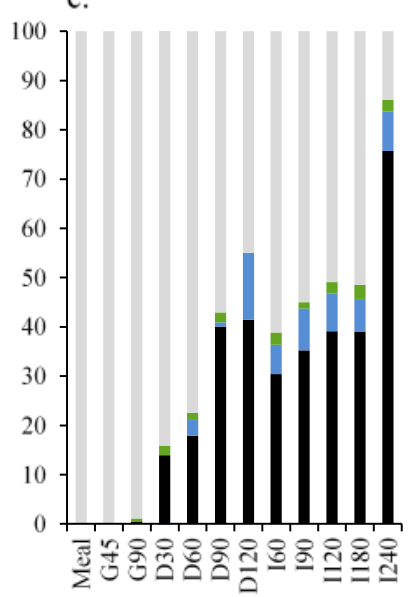

Figure 6.3. Evolution of the relative molar fractions of the different lipid species (FFA: black; MAG: dark blue; DAG: green; TAG: grey) during dynamic in vitro digestion of a) free emulsion meal, b) free emulsion meal with empty beads, and c) encapsulated emulsion meal in the gastric (G), duodenal (D), and distal small intestinal (I) compartment at the indicated time points in minutes. Mean values of independent digestions are shown $(n=3)$.

\subsubsection{Fatty acid incorporation in mixed micelles in the intestinal compartments}

\section{Absorbable fraction from free emulsion}

As FFA or MAG will be absorbed only after their solubilisation in mixed micelles (Golding et al., 2011; Maldonado-Valderrama, Wilde, Macierzanka, \& Mackie, 2011), it is of relevance to estimate their quantities throughout the small intestine. Figure 6.4 shows the evolution of fatty acids (FA) from the absorbable lipid species (i.e., FFAs and MAGs) in mixed micelles during dynamic in vitro digestion of free emulsion meal. In the duodenal compartment, the concentration of FAs in the mixed micelles increased sharply (Figure 6.4a), indicating that the reaction rate was much higher than the overall dilution applied to keep the duodenal volume at a maximum of $50 \mathrm{~mL}$. 
The bioaccessibility (percentage FA in micellar phase relative to FA in total lipids) was also calculated (Figure 6.4b). Due to the dynamic amount of TAG that is used in this calculation, it was found that about half of the FAs were bioaccessible in the duodenal phase and higher levels were reached in the distal small intestinal compartment (60-80\%). In both compartments, all released FFAs (Figure 6.2a) seem to be incorporated in mixed micelles keeping the bioaccessibility constant (i.e., absorbable lipid fraction). This could indicate that solubilisation in mixed micelles was not rate limiting, which is in agreement with previous work (Giang et al., 2016).
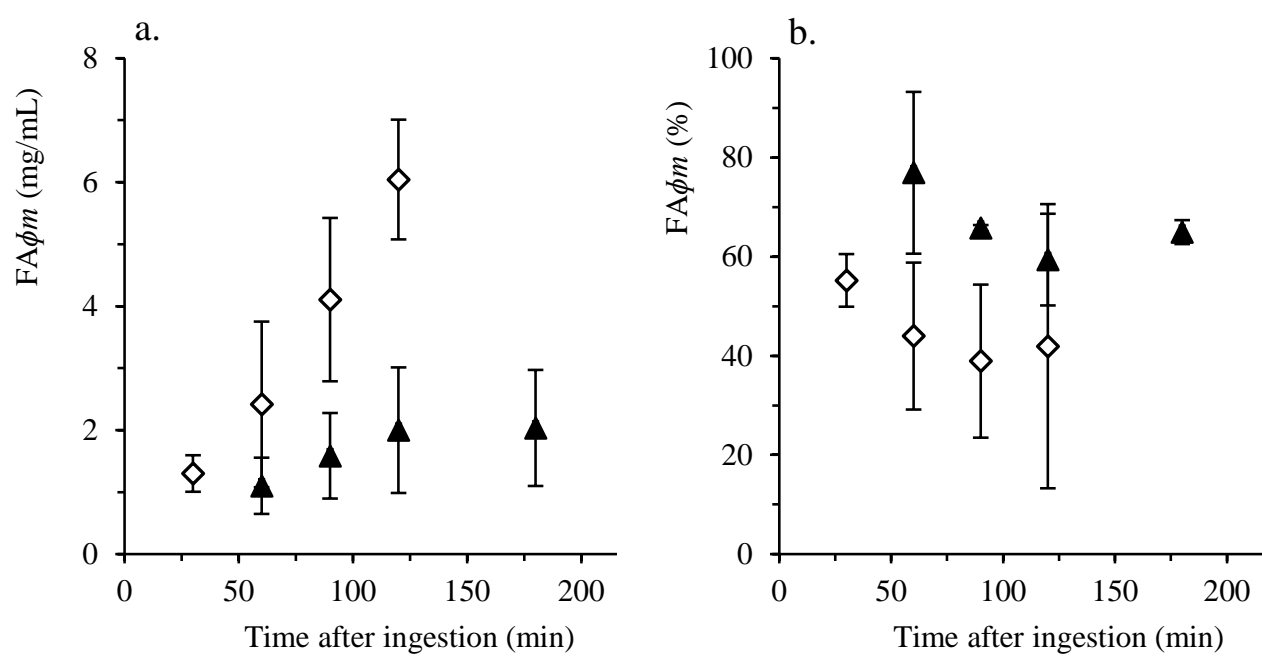

Figure 6.4. Evolution of fatty acids (FA) in mixed micelles $\left(\phi_{m}\right)$ during dynamic in vitro digestion of free emulsion meal in the duodenal (empty diamonds) and distal small intestinal (black triangles) compartment, given as a) absolute concentrations in the micellar phase, and b) bioaccessibility (percentage FA in micellar phase relative to FA in total lipids). Mean values with standard deviations are shown $(n=3)$.

Safflower oil contains mainly linoleic acid (C18:2n-6; 73\%), but also oleic acid (C18:1n9; 14\%), palmitic acid (C16; 7\%), little stearic acid (C18:0; 3\%), and a broad range of other fatty acids in low amounts (see Supplementary Table 6.3). Since the kinetics of lipolysis and solubilisation depend on the type of fatty acid (Giang et al., 2016; Zhu, Ye, Verrier, \& Singh, 2013), we checked the evolution of the individual FAs (Figure 6.5), and found no clear selectivity in incorporation in mixed micelles as observed for long chain polyunsaturated FAs. 
a.

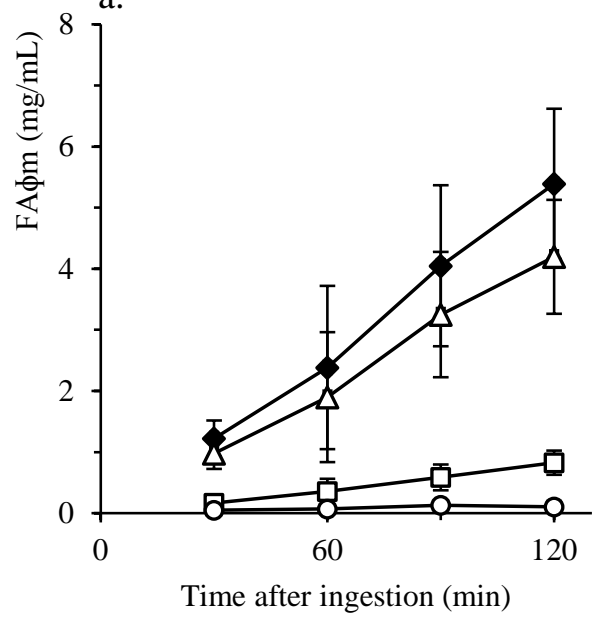

b.

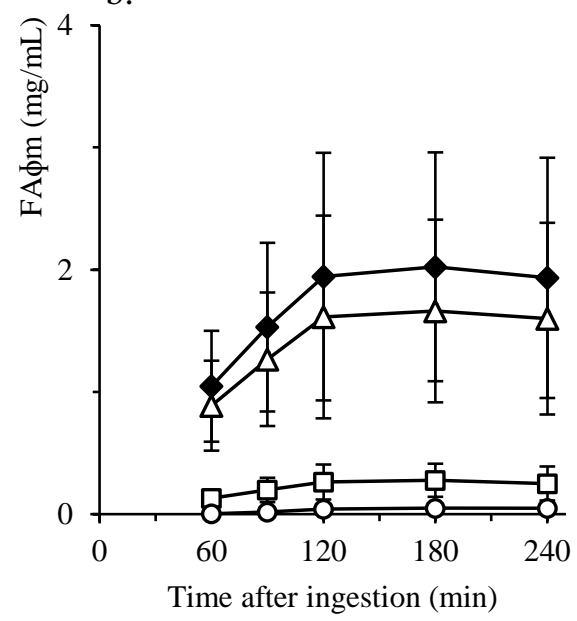

Figure 6.5. Evolution of fatty acid (FA) concentrations in mixed micelles ( $\left.\phi_{\mathrm{m}}\right)$ during dynamic in vitro digestion of free emulsion meal. The total FA concentration (filled diamond) is given as well as individual concentrations of C18:2 (empty triangle), C18:1 (empty square), and C16:0 (empty circle); in the a) duodenal and b) distal small intestinal compartment. Mean values with standard deviations are shown $(n=3)$.

\section{Absorbable fraction from emulsion-alginate beads}

Figure 6.6a shows the micellar FA concentration during in vitro digestion of the three meals. In the case of encapsulated emulsion meal, much less FA was micellarized in the duodenal (average bioaccessibility of 5\%) and distal fluid (average bioaccessibility of 3\%) compared to free emulsion meals (average bioaccessibility of $46-67 \%$ in both compartments) independently of the presence of empty beads (see Supplementary Table 6.4 for all data). Since lipolysis of encapsulated emulsion did take place in the end of the distal small intestinal compartment (Figure 6.2), the observed low amount of micellar FA points to a delay in the solubilisation step after the release of FFAs. These findings indicate not only a delayed digestion (i.e., release of FFAs) of the encapsulated emulsion meal compared to both free emulsion meals, but also a delayed bioaccessibility (i.e., solubilisation of FFAs in mixed micelles). This indicates that although the triacylglycerols may be hydrolysed inside the gel beads, their reaction products are not registered in the aqueous part of the digestive media. This is caused by the diffusive distance through the gel beads, which can be related to the digestion products as such, or to micelles that contain these components but are present inside the beads. Either way, the effect that this creates is beneficial for reduced release, and thus of great relevance for a product that can be used in weight-management strategies. 
a.

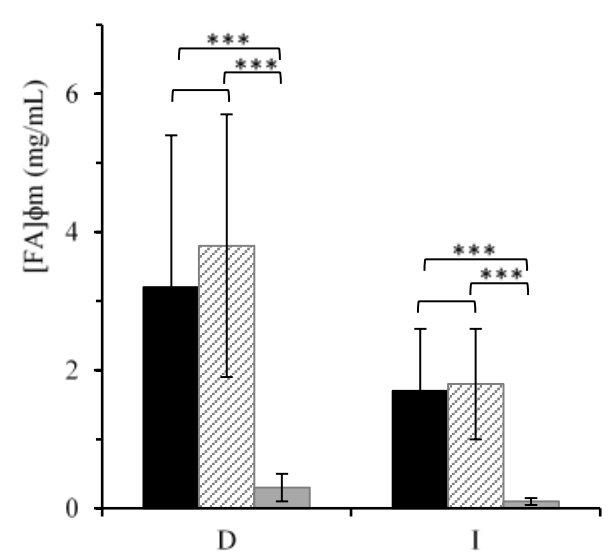

b.

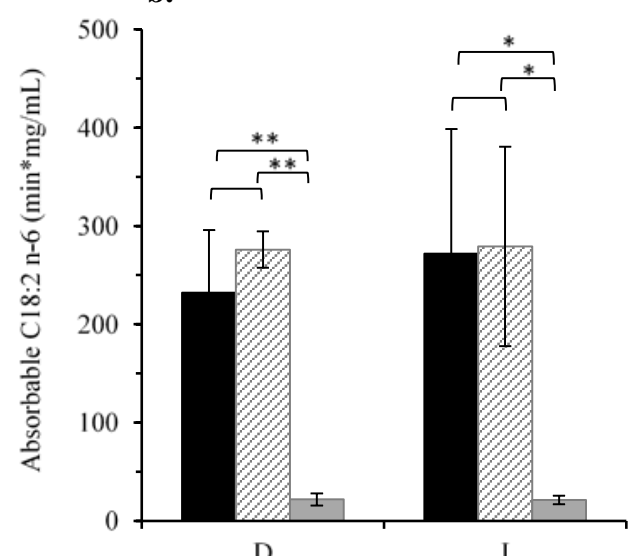

Figure 6.6. Average fatty acid (FA) concentration in the micellar phase ( $\left.\phi_{\mathrm{m}}\right)$ (panel a), during in vitro digestion of free emulsion meal (black), free emulsion meal with empty beads (white strike), encapsulated emulsion meal (grey), in the duodenal (D), and distal small intestinal (I) compartment. Panel b shows the absorbable amount of linoleic acid (C18:2 n-6) in the whole compartment (obtained by integrating the data). Mean values with standard deviations are shown $(n=3)$. Significant differences in the Tukey HSD Post Hoc test are indicated with * $(p<0.1),{ }^{* *}(p<0.01)$, and $* * *(p<0.001)$.

Since linoleic acid (18:2 n-6) is known to most strongly induce the satiety signals (Maljaars et al., 2009), the absorbable amount of 18:2 n-6 in the whole duodenal and distal small intestinal compartment was calculated for the three meals (Figure 6.6b). The results show that non-encapsulated 18:2 n-6 (black and strike bars) will be mainly absorbed in the proximal small intestine, making it unable to induce a brake in the distal part of the intestine. On the other hand, encapsulated 18:2 n-6 (grey bar) will not be absorbed in the proximal small and may be released later in the distal small intestine, and hence, induce ileal brake activation.

We are aware of the fact that in vivo digestion is even more complex than the system we used, including physiological regulation mechanisms such as back-control, and for example an intestinal mucus layer (Arranz, Corredig, \& Guri, 2016), which cannot be included in the DIDGI. So as stated by Oosterveld and co-workers: care must be taken to translate these results to the in vivo reality (Oosterveld et al., 2016), but the great differences found make us hopeful that we have identified an encapsulation method that could induce an ileal brake after ingestion, and that can also be tuned further if needed. 


\subsection{CONCLUSIONS}

Our objective was to assess the behaviour of free emulsions and emulsion-alginate beads under dynamic digestion conditions to test their potentiality to induce the ileal brake. Lipolysis of free emulsions occurred quickly, corresponding to proximal digestion, and since incorporation of absorbable lipid species in mixed micelles was not rate limiting this points to early absorption. Encapsulation of emulsions in calcium-alginate beads with appropriate size and porosity provided protection via physical entrapment. This retarded lipolysis to the distal small intestine and delayed bioaccessibility, which is in line with the requirements for induction of the ileal brake. Our findings do not only increase the understanding of the dynamics of lipid digestion, but can also be of relevance in the design of a lipid carrier in the context of a food-based approach for long-term weight management.

\section{Acknowledgements}

We would like to thank Lucie Ribourg (INRA, France) for technical assistance; and Freddy Troost and Ad Masclee for their insight in the physiological situation. Research presented in this publication was financially supported by the Graduate School VLAG; the Dr. ir. Aalt Dijkhuizen Fund financially supported Meinou Corstens’ travel to INRA.

\subsection{SUPPLEMENTARY INFORMATION}
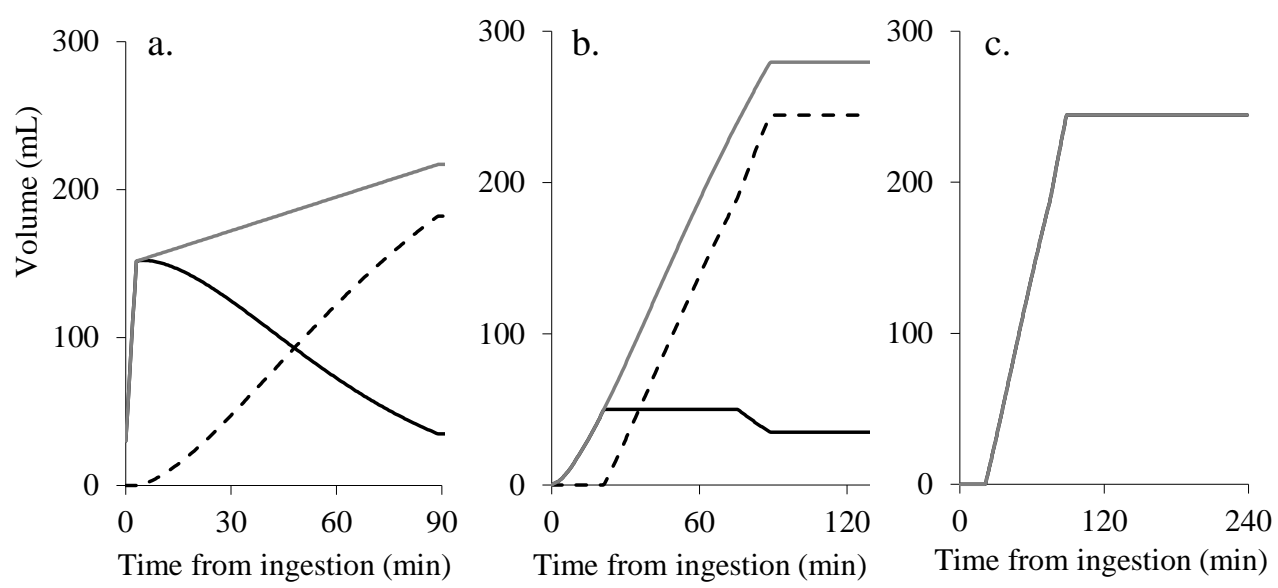

Supplementary Figure 6.7. Evolution of volumes: remaining (black line), emptied (dotted black line), and total introduced volume (remaining + emptied; grey line), in the a) gastric, b) duodenal, and c) distal small intestinal compartment. 
Supplementary Table 6.3. Fatty acid composition (\%) of safflower oil.

\begin{tabular}{lc}
\hline Fatty acid & $\mathbf{\%}$ \\
\hline C14 & 0.3 \\
C15 & 0.0 \\
C16 & 7.4 \\
C16:1n-9 & 0.0 \\
C16:1n-7 & 0.0 \\
c17:1 & 0.0 \\
C18 & 3.0 \\
C18:1n-9 & 14.2 \\
C18:1n-7 & 0.6 \\
C18:2n-6 & 72.6 \\
C18:3n-6 & 0.1 \\
C18:3n-3 & 0.1 \\
C20 & 0.4 \\
C20:1n-9 & 0.3 \\
C20:2 & 0.0 \\
C20:3 & 0.0 \\
C20:4 & 0.0 \\
C22 & 0.3 \\
C22:1 & 0.5 \\
\hline
\end{tabular}

Supplementary Table 6.4. Fatty acid (FA) concentration in the total lipid extract (TL) and micellar phase $\left(\phi_{m}\right)$, during in vitro digestion of fat-free yogurts enriched with free emulsion (EM), free emulsion and empty beads (EM+EB), emulsion-alginate beads (EM-A beads), in the duodenal (D), and distal small intestinal (I) compartment. Mean values with standard deviations are given $(n=3)$. A common letter indicates no significant difference between meals using Tukey HSD Post Hoc test $(p<0.001)$.

\begin{tabular}{|c|c|c|c|c|c|c|c|}
\hline & \multicolumn{2}{|c|}{$[\mathrm{FA}]_{\mathrm{TL}}(\mathrm{mg} / \mathrm{mL})$} & \multicolumn{2}{|c|}{$[\mathrm{FA}]_{\phi \mathrm{m}}(\mathrm{mg} / \mathrm{mL})$} & \multicolumn{2}{|l|}{$\mathrm{FA}_{\phi \mathrm{m}}(\%)$} \\
\hline & & Mean (sd) & Sig. & Mean (sd) & Sig. & Mean (sd) & Sig. \\
\hline \multirow[t]{3}{*}{$\mathrm{D}$} & EM & 9.2 (10.8) & & $3.2^{\mathrm{a}}(2.2)$ & & $45.9^{c}(19.0)$ & \\
\hline & $\mathrm{EM}+\mathrm{EB}$ & $5.7(2.6)$ & 0.351 & $3.8^{\mathrm{a}}(1.9)$ & 0.000 & $66.6^{\mathrm{c}}(13.3)$ & 0.000 \\
\hline & EM-A beads & $5.8(3.5)$ & & $0.3(0.2)$ & & $5.0 \quad(2.7)$ & \\
\hline \multirow[t]{3}{*}{ I } & EM & $2.6(1.4)$ & & $1.7^{\mathrm{b}}(0.9)$ & & $65.4^{\mathrm{d}}(11.7)$ & \\
\hline & $\mathrm{EM}+\mathrm{EB}$ & $3.4(1.1)$ & 0.327 & $1.8^{\mathrm{b}}(0.8)$ & 0.000 & $53.3^{d}(13.2)$ & 0.000 \\
\hline & EM-A beads & 3.2 (1.7) & & $0.1(0.05)$ & & 3.3 (1.8) & \\
\hline
\end{tabular}




\subsection{REFERENCES}

Alleleyn, A. M. E., van Avesaat, M., Troost, F. J., \& Masclee, A. A. M. (2016). Gastrointestinal Nutrient Infusion Site and Eating Behavior: Evidence for A Proximal to Distal Gradient within the Small Intestine? Nutrients, 8(3), 117-132.

Armand, M. (2007). Lipases and lipolysis in the human digestive tract: where do we stand? Current Opinion in Clinical Nutrition and Metabolic Care, 10(2), 156-64.

Arranz, E., Corredig, M., \& Guri, A. (2016). Designing food delivery systems: challenges related to the in vitro methods employed to determine the fate of bioactives in the gut. Food \& Function, 7(8), 3319 3336.

Berrada, N., Lemeland, J. F., Laroche, G., Thouvenot, P., \& Piaia, M. (1991). Bifidobacterium from fermented milks: survival during gastric transit. Journal of Dairy Science, 74(2), 409-413.

Blanquet, S., Zeijdner, E., Beyssac, E., Meunier, J. P., Denis, S., Havenaar, R., \& Alric, M. (2004). A dynamic arificial gastrointestinal system for studying the behavior of orally administrated drug dosage forms under various physiological conditions. Pharmaceutical Research, 21(4), 585-591.

Calbet, J. A. L., \& MacLean, D. A. (1997). Role of caloric content on gastric emptying in humans. The Journal of Physiology, 498(2), 553-559.

Chu, B.-S., Rich, G. T., Ridout, M. J., Faulks, R. M., Wickham, M. S. J., \& Wilde, P. J. (2009). Modulating pancreatic lipase activity with galactolipids: effects of emulsion interfacial composition. Langmuir, 25(16), 9352-9360.

Corstens, M. N., Berton-Carabin, C. C., de Vries, R., Troost, F. J., Masclee, A. A. M., \& Schroën, K. (2017). Food-grade micro-encapsulation systems that may induce satiety via delayed lipolysis: A Review. Critical Reviews in Food Science and Nutrition, 57(10), 2218-2244.

Corstens, M. N., Berton-Carabin, C. C., Elichiry-Ortiz, P. T., Hol, K., Troost, F. J., Masclee, A. A. M., \& Schroën, K. (2017). Emulsion-alginate beads designed to control in vitro intestinal lipolysis: Towards appetite-control. Journal of Functional Foods, 34(July), 319-328.

Corstens, M. N., Berton-Carabin, C. C., Kester, A., Fokkink, R., van den Broek, J. M., de Vries, R., ... Schroën, K. (2017). Destabilization of multilayered interfaces in digestive conditions limits their ability to prevent lipolysis in emulsions. Food Structure, 12(April), 54-63.

Couëdelo, L., Amara, S., Lecomte, M., Meugnier, E., Monteil, J., Fonseca, L., ... Vaysse, C. (2015). Impact of various emulsifiers on ALA bioavailability and chylomicron synthesis through changes in gastrointestinal lipolysis. Food Function, 6(5), 1726-1735.

Cummings, D. E., \& Overduin, J. (2007). Gastrointestinal regulation of food intake. Journal of Clinical Investigation, 117(1), 13-23.

de Oliveira, S. C., Deglaire, A., Ménard, O., Bellanger, A., Rousseau, F., Henry, G., ... Bourlieu, C. (2016). Holder pasteurization impacts the proteolysis, lipolysis and disintegration of human milk under in vitro dynamic term newborn digestion. Food Research International, 88, 263-275.

Dekkers, B., Kolodziejczyk, E., Acquistapace, S., Engmann, J., \& Wooster, T. J. (2016). Impact of gastric $\mathrm{pH}$ profiles on the proteolytic digestion of mixed $\beta 1 \mathrm{~g}$-Xanthan biopolymer gels. Food Function, 7(1), 58-68.

Elashoff, J., Reedy, T., \& Meyer, J. (1982). Analysis of gastric emptying data. Gastroenterology, 83(6), 1306-12.

Giang, T. M., Gaucel, S., Brestaz, P., Anton, M., Meynier, A., Trelea, I. C., \& Le Feunteun, S. (2016). Dynamic modeling of in vitro lipid digestion: Individual fatty acid release and bioaccessibility kinetics. Food Chemistry, 194, 1180-1188.

Golding, M., Wooster, T. J., Day, L., Xu, M., Lundin, L., Keogh, J., \& Clifton, P. (2011). Impact of gastric structuring on the lipolysis of emulsified lipids. Soft Matter, 7, 3513-3523.

Guerra, A., Etienne-Mesmin, L., Livrelli, V., Denis, S., Blanquet-Diot, S., \& Alric, M. (2012). Relevance and challenges in modeling human gastric and small intestinal digestion. Trends in Biotechnology, 30(11), 591-600.

Guillemin, H., Perret, B., Ménard, O., \& Picque, D. (2010). Logiciel StoRM V2 (Stomach and duodenum Regulation and Monitoring). IDDN.FR.001.2300009.001.R.P.2010.000.31235.

Hara, A., \& Radin, N. S. (1978). Lipid extraction of tissues with a low-toxicity solvent. Analytical Biochemistry, 90(1), 420-426.

Hu, M., Li, Y., Decker, E. A., Xiao, H., \& McClements, D. J. (2011). Impact of Layer Structure on Physical Stability and Lipase Digestibility of Lipid Droplets Coated by Biopolymer Nanolaminated Coatings. Food Biophysics, 6, 37-48. 
Kenmogne-Domguia, H.B., Meynier, A., Viau, M., Llamas, G., Genot, C. (2012). Gastric conditions control both the evolution of the organization of protein-stabilized emulsions and the kinetic of lipolysis during in vitro digestion. Food \& Function, 3(12), 1302-9.

Klinkesorn, U., \& McClements, D. J. (2010). Impact of lipase, bile salts, and polysaccharides on properties and digestibility of tuna oil multilayer emulsions stabilized by lecithin-chitosan. Food Biophysics, 5(2), 73-81.

Kong, F., \& Singh, R. P. (2008). Disintegration of solid foods in human stomach. Journal of Food Science, 73(5), 67-80.

Li, Y., Hu, M., Du, Y., Xiao, H., \& McClements, D. J. (2011). Control of lipase digestibility of emulsified lipids by encapsulation within calcium alginate beads. Food Hydrocolloids, 25(1), 122-130.

Lundin, L., Golding, M., \& Wooster, T. J. (2008). Understanding food structure and function in developing food for appetite control. Nutrition and Dietetics, 65(Suppl. 3), S79-85.

Malagelada, J. R., Go, V. L., \& Summerskill, W. H. (1979). Different gastric, pancreatic, and biliary responses to solid-liquid or homogenized meals. Digestive Diseases and Sciences, 24, 101-110.

Maldonado-Valderrama, J., Wilde, P., Macierzanka, A., \& Mackie, A. (2011). The role of bile salts in digestion. Advances in Colloid and Interface Science, 165, 36-46.

Maljaars, P. W. J., Peters, H. P. F., Mela, D. J., \& Masclee, A. A. M. (2008). Ileal brake: a sensible food target for appetite control. A review. Physiology \& Behavior, 95(3), 271-281.

Maljaars, P. W. J., Romeyn, E. A., Haddeman, E., Peters, H. P. F., \& Masclee, A. A. M. (2009). Effect of fat saturation on satiety, hormone release, and food intake. American Journal of Clinical Nutrition, 89, 1019-1024.

Ménard, O., Cattenoz, T., Guillemin, H., Souchon, I., Deglaire, A., Dupont, D., \& Picque, D. (2014). Validation of a new in vitro dynamic system to simulate infant digestion. Food Chemistry, 145, 10391045.

Meynier, A., Leborgne, C., Viau, M., Schuck, P., Guichardant, M., Rannou, C., \& Anton, M. (2014). N-3 fatty acid enriched eggs and production of egg yolk powders: An increased risk of lipid oxidation? Food Chemistry, 153, 94-100.

Minekus, M., Marteau, P., Havenaar, R., \& Huis in 't Veld, J. H. J. (1995). A multicompartmental dynamic computer-controlled model simulating the stomach and small intestine. Alternatives to Laboratory Animals, 23, 197-209.

Morrison, W. R., \& Smith, L. M. (1964). Preparation of fatty acid methyl esters and dimethyl acetals from lipids with boron trifluoride methanol. Journal of Lipid Research, 5, 600-608.

Mun, S., Decker, E. A., Park, Y., Weiss, J., \& McClements, D. J. (2006). Influence of Interfacial Composition on in vitro Digestibility of Emulsified Lipids: Potential Mechanism for Chitosan's Ability to Inhibit Fat Digestion. Food Biophysics, 1(1), 21-29.

Oosterveld, A., Minekus, M., Bomhof, E., Zoet, F. D., \& van Aken, G. A. (2016). Effects of inhomogeneity on triglyceride digestion of emulsions using an in vitro digestion model (Tiny TIM). Food Function, 7, 2979-2995.

Sams, L., Paume, J., Giallo, J., \& Carrière, F. (2016). Relevant pH and lipase for in vitro models of gastric digestion. Food \& Function, 7, 30-45.

Sarkar, A., Juan, J.-M., Kolodziejczyk, E., Acquistapace, S., Donato-Capel, L., \& Wooster, T. J. (2015). Impact of protein gel porosity on the digestion of lipid emulsions. Journal of Agricultural and Food Chemistry, 63(40), 8829-8837.

Sarkar, A., Murray, B., Holmes, M., Ettelaie, R., Abdalla, A., \& Yang, X. (2016). In vitro digestion of Pickering emulsions stabilized by soft whey protein microgel particles: influence of thermal treatment. Soft Matter, 12(15), 3558-69.

Tzoumaki, M. V, Moschakis, T., Scholten, E., \& Biliaderis, C. G. (2013). In vitro lipid digestion of chitin nanocrystal stabilized o/w emulsions. Food \& Function, 4(1), 121-9.

van Avesaat, M., Troost, F. J., Ripken, D., Hendriks, H. F., \& Masclee, A. A. M. (2015). Ileal brake activation: macronutrient specific effects on eating behavior? International Journal of Obesity, 39(April), 235243.

Van Citters, G. W., \& Lin, H. C. (2006). Ileal brake: neuropeptidergic control of intestinal transit. Current Gastroenterology Reports, 8(5), 367-73.

Verhoeckx, K., Cotter, P., Kleiveland, C., Lea, T., Mackie, A., \& Requena, T. (2015). The Impact of Food Bioactives on Health. Springer Open.

Villemejane, C., Denis, S., Marsset-Baglieri, A., Alric, M., Aymard, P., \& Michon, C. (2016). In vitro digestion of short-dough biscuits enriched in proteins and/or fibres using a multi-compartmental and dynamic system (2): Protein and starch hydrolyses. Food Chemistry, 190, 164-172. 
Wilcox, M. D., Brownlee, I. A., Richardson, J. C., Dettmar, P. W., \& Pearson, J. P. (2014). The modulation of pancreatic lipase activity by alginates. Food Chemistry, 146, 479-484.

Wulff-pérez, M., Vicente, J. De, Martín-Rodríguez, A., \& Gálvez-Ruiz, M. J. (2012). Controlling lipolysis through steric surfactants: New insights on the controlled degradation of submicron emulsions after oral and intravenous administration. International Journal of Pharmaceutics, 423(2), 161-166.

Zeeb, B., Lopez-Pena, C. L., Weiss, J., \& McClements, D. J. (2015). Controlling lipid digestion using enzyme-induced crosslinking of biopolymer interfacial layers in multilayer emulsions. Food Hydrocolloids, 46, 125-133.

Zhang, Z., Zhang, R., Zou, L., Chen, L., Ahmed, Y., Bishri, W. Al, .. McClements, D. J. (2016). Encapsulation of curcumin in polysaccharide-based hydrogel beads : Impact of bead type on lipid digestion and curcumin bioaccessibility. Food Hydrocolloids, 58, 160-170.

Zhu, X., Ye, A., Verrier, T., \& Singh, H. (2013). Free fatty acid profiles of emulsified lipids during in vitro digestion with pancreatic lipase. Food Chemistry, 139(1-4), 398-404. 
Chapter 6 


\title{
Chapter 7
}

\section{Emulsion-alginate beads:}

\author{
the effect on food intake
}

This chapter is submitted as Corstens, M.N., Troost, F.J., Alleleyn, A.M.E., Klaassen, T., Berton-Carabin, C.C., Schroën, K., Masclee, A.A.M. Encapsulation of lipid as emulsionalginate beads reduces food intake: a randomized placebo-controlled cross-over trial in overweight adults. 


\section{ABSTRACT}

Background: The ileal brake mechanism, which induces satiety after sensing nutrients or their metabolites in the ileum, is considered a potent feedback mechanism. Triggering this mechanism through oro-ileal lipid delivery could be the basis for a weight management strategy. We hypothesize that emulsion-alginate beads prevent proximal digestion and absorption of the encapsulated lipid, and that its more distal (ileal) release induces feelings of satiety and reduces food intake.

Objective: This study investigates the ability of ingested emulsion-alginate beads to decrease food intake, and to increase feelings of satiety without increasing gastrointestinal (GI) symptoms.

Design: Thirty-three healthy overweight volunteers (mean age: 43 yrs.; mean BMI: 27.7 $\mathrm{kg} \cdot \mathrm{m}^{-2} ; 14$ male) received two treatments in a randomized placebo-controlled trial with crossover design. Test days started with intake of a standardized small breakfast $(t=0)$, followed by a yogurt ( $\mathrm{t}=90 \mathrm{~min}$ ) that contained either encapsulated lipid as emulsion-alginate beads (active) or an equicaloric mixture of non-encapsulated nutrients with similar sensory properties (control). Feelings of satiety and GI symptoms were monitored prior to and after consumption of the yogurt, and food intake was measured during ad libitum pasta meal consumption $(\mathrm{t}=210 \mathrm{~min})$.

Results: Food intake was significantly reduced with $51 \pm 20 \mathrm{kcal}(p=0.016)$ after intake of the active yogurt $(770 \pm 38 \mathrm{kcal})$ compared to the control $(821 \pm 40 \mathrm{kcal})$. Satiety was increased after intake of the active yogurt compared to the control ( $p=0.013$ ), but fullness, hunger, desire to eat, and desire to snack were not altered by the intervention.

Conclusions: Ingested emulsion-alginate beads reduce food intake, suggesting that release of encapsulated lipids in the distal small intestine activated the ileal brake. These findings have important implications for understanding satiety mechanisms, and moreover, the development of a product for weight management. 


\subsection{INTRODUCTION}

The worldwide rapid expansion of obesity demands new non-invasive treatment strategies. One option is via dietary interventions that regulate food intake by inducing feelings of satiety (Camilleri \& Sweetser, 2007). Amongst other gastrointestinal (GI) processes involved in food intake regulation, nutrient sensing in the small intestine can induce negative feedback signals to the proximal GI tract and central nervous system to inhibit digestion, appetite sensations and food intake, and to increase feelings of satiety and satiation (Maljaars, Peters, Mela, \& Masclee, 2008; van Avesaat, Troost, Ripken, Hendriks, \& Masclee, 2015). The ileum is believed to provide the strongest signal, i.e., 'the ileal brake' (Alleleyn, van Avesaat, Troost, \& Masclee, 2016; Maljaars et al., 2011). Ileal brake activation has been proven via direct intra-ileal infusion of macronutrients, applying naso-intestinal feeding catheters in human volunteers (Alleleyn et al., 2016). Already thirty years ago ileal infusion of lipid was shown to reduce food intake (Welch, Saunders, \& Read, 1985; Welch, Sepple, \& Read, 1988). However, in order to use these effects in a regular daily setting as part of a long-term weight management strategy, ileal lipid delivery needs to be achieved after oral ingestion, without need for intubation.

The degradation products of lipid digestion (i.e., free fatty acids and monoacylglycerols) activate the ileal brake more than the intact lipids itself (Camilleri \& Sweetser, 2007; Little et al., 2007; Van Citters \& Lin, 1999), but delivery of lipid degradation products to the ileum requires lipolysis without proximal absorption. Under physiological conditions, orally ingested lipids are not likely to deliver discernible amounts of such degradation products in the ileum due to a range of processes that allow efficient lipolysis and absorption in the proximal small intestine (Bakala N’Goma, Amara, Dridi, Jannin, \& Carrière, 2012). To enable this, lipids need to be protected in a carrier type that remains intact under the acidic conditions in the stomach, and that slowly releases degradation products of lipid digestion in the distal small intestine (Camilleri \& Sweetser, 2007).

Many attempts have been made to control lipid digestion, mainly through designing a protective interfacial structure around nano- or micron-sized emulsion droplets (Corstens, Berton-Carabin, de Vries, et al., 2017) but this has proven to be ineffective, even for multilayered structures (Corstens, Berton-Carabin, Kester, et al., 2017; Corstens, Osorio Caltenco, de Vries, Schroën, \& Berton-Carabin, 2017). This has lead us to conclude that in order to truly control lipolysis, an approach via interfacial design is not sufficient to achieve the desired effect. The focus should be on controlling the exposure of the lipids to digestive enzymes in the GI tract, by incorporating these in a large structure. Emulsion-filled calciumalginate beads (named emulsion-alginate beads from here on) have been suggested for this purpose, as the indigestibility of alginate preserves its structure, and the $\mathrm{pH}$-dependent response of such beads favours lipase diffusion towards encapsulated emulsified lipids in the 
small intestine; namely, the beads shrink under acidic conditions and have a smaller mesh size, thereby protecting the encapsulated emulsion in the stomach. At increasing $\mathrm{pH}$, such as in the small intestine, the beads swell and increase in mesh size (Corstens, Berton-Carabin, Elichiry-Ortiz, et al., 2017; Li, Hu, Du, Xiao, \& McClements, 2011; Rayment et al., 2009; Wright et al., 2009; Zeeb, Saberi, Weiss, \& McClements, 2015). Further, these beads are expected to have a prolonged residence time in the ileum, since alginate beads (without lipid) have been shown to have a longer transit time in the human ileum compared to that in the duodenum and jejunum, respectively (Hoad et al., 2009).

Emulsion-alginate beads have been shown to improve the integrity of the encapsulated lipid (in terms of microscopic observation) during gastric transit and delay intestinal lipid absorption compared to free emulsion droplets or emulsion microclusters in rats (Li, Kim, Park, \& McClements, 2012). These findings were in line with a human trial in which large $(0.5 \mathrm{~cm})$ oil-core alginate-shell capsules were shown to delay intestinal lipolysis and absorption (Hoad et al., 2011). For the current human intervention study, small alginate beads $(0.5-1.2 \mathrm{~mm})$ that encapsulate emulsion droplets $\left(d_{32} \sim 25 \mu \mathrm{m}\right.$; safflower oil as satiety inducer (Maljaars, Romeyn, Haddeman, Peters, \& Masclee, 2009)) were developed to delay intestinal lipolysis, and were shown to control in vitro lipolysis after prior gastric incubation by altering bead and mesh size (Corstens, Berton-Carabin, Elichiry-Ortiz, et al., 2017). Monitoring dynamic in vitro lipolysis of such emulsion-alginate beads confirmed lipolysis at time scales relevant for delivery to the more distal part of the small intestine, whereas lipolysis of nonencapsulated emulsions occurred too fast (Corstens, Berton-Carabin, Schroën, et al., 2017).

When designed properly, emulsion-alginate beads are expected to protect the encapsulated lipid while passing through the stomach, and to efficiently deliver lipid degradation products in the distal small intestine. The present work is an explorative study to confirm the concept of ileal brake activation in overweight individuals with these emulsion-alginate beads ingested in yogurt, through assessing subsequent feelings of satiety and food intake during ad libitum meal consumption in a randomized cross-over study in healthy overweight adults, compared to a control yogurt that contains an equicaloric mixture of non-encapsulated nutrients with similar sensory properties.

\subsection{SUBJECTS, MATERIALS AND METHODS}

\subsubsection{Materials}

Safflower oil was purchased from De Wit Specialty oils (19200 Safflower Oil High Linoleic Refined; The Netherlands), whey protein isolate (WPI) from Davisco Foods International (BiPro, purity 97.5\%; Eden Prairie, MN, USA), calcium chloride from Boom B.V. (Prills Foodgrade; Meppel, The Netherlands), and sodium alginate (W201502) from Sigma Aldrich 
(St. Louis, MO, USA). Fat-free yogurt, bread, marmalade, lemon juice, and the pasta meals were purchased at a local supermarket. All materials were certified for food-grade use, and used directly without further purification.

\subsubsection{Preparation of the enriched yogurts}

Commercial fat-free yogurt (Campina magere yoghurt, The Netherlands; energy density per 100 g: 39 kcal, 0 g lipid, 4 g carbohydrates, 4.7 g protein) was enriched with emulsified safflower oil (6 g) being either encapsulated in alginate beads (active) or non-encapsulated (control; in presence of 'empty' beads), as schematically shown in Figure 7.1.

Emulsion. Safflower oil was added to a WPI solution (20 wt $\%$ oil, $10 \mathrm{~g} \cdot \mathrm{L}^{-1} \mathrm{WPI}$ in the aqueous phase) and mixed using a rotor stator homogenizer (Ika T18 basic Ultra-Turrax homogenizer equipped with a S18N-19G dispersion tool, Staufen, Germany) for 5 min at $13,000 \mathrm{rpm}$, to obtain an emulsion with a droplet size $\left(d_{32}\right)$ of $25 \mu \mathrm{m}$.

Emulsion-alginate beads. Fresh emulsion ( $<5$ min after production) was mixed with an alginate solution $\left(36 \mathrm{~g} \cdot \mathrm{L}^{-1}\right)$ in volume ratio 1:1 (final composition: $10 \mathrm{wt} \%$ oil, $20 \mathrm{~g} \cdot \mathrm{L}^{-1}$ alginate in the aqueous phase, Table 7.1). This alginate-emulsion mixture was added dropwise to a gently stirred calcium bath $\left(5 \mathrm{wt} \% \mathrm{CaCl}_{2}\right.$ with some drops of lemon juice to lower the $\mathrm{pH}$ and fully dissolve the calcium), as described previously (Corstens, BertonCarabin, Elichiry-Ortiz, et al., 2017). The needle tip (inner diameter $0.41 \mathrm{~mm}$; Nordson EFD, U.K.) was positioned 5-6 $\mathrm{cm}$ above the calcium bath and a syringe pump was used to regulate the flow rate $\left(3.0 \mathrm{~mL} \cdot \mathrm{min}^{-1}\right)$. To control the bead size, an airflow was applied (Jun-Air 86R4B compressor, Benton Harbor, MI, USA) passing the needle in a homogeneous way, resulting in a bead size of $1.10 \pm 0.05 \mathrm{~mm}$. After production, the emulsion-alginate beads were stored at $4{ }^{\circ} \mathrm{C}$ at least overnight to harden completely and not more than four days to prevent microbial spoilage. On the test day, the beads were filtered and washed with tap water (10-times volume, > $10 \mathrm{~min}$ ), and filtered again to obtain weighable beads.

Empty beads. Alginate beads were produced from $20 \mathrm{~g} \cdot \mathrm{L}^{-1}$ alginate solution in the same way as described above, but not mixed with emulsion. They had a bead size of $1.21 \pm 0.02 \mathrm{~mm}$, similar to the emulsion-alginate beads. These beads were also stored at $4{ }^{\circ} \mathrm{C}$ for 1 to 4 days, and washed and filtered before use.

Active yogurt. Fat-free yogurt (120 g) was mixed with $60 \mathrm{~g}$ emulsion-alginate beads (containing 6 g safflower oil). The composition was matched to the control yogurt by adding $24 \mathrm{~g}$ water. The active yogurts contained $105 \mathrm{kcal}$; of which $51 \mathrm{kcal} \%$ come from the encapsulated lipid (Table 7.1).

Control yogurt. Fat-free yogurt (120 g) was mixed with $30 \mathrm{~g}$ emulsion (containing $6 \mathrm{~g}$ safflower oil), and 54 g empty beads to match the composition and sensory properties of the 
active yogurt. Therefore, the control yogurt was an equicaloric mixture of non-encapsulated nutrients and also contained $105 \mathrm{kcal}$ of which $51 \mathrm{kcal} \%$ come from the non-encapsulated lipid (Table 7.1).

Table 7.1. Overview of the composition and caloric content of the studied formulations.

\begin{tabular}{|c|c|c|}
\hline Name & Active & Control \\
\hline Safflower oil (g) & 6.0 & 6.0 \\
\hline Whey protein (g) & $\leq 0.24$ & 0.24 \\
\hline Alginate (g) & 1.08 & 1.08 \\
\hline Water* (g) & 76.9 & 76.9 \\
\hline Yogurt (g) & 120 & 120 \\
\hline Total (g) & 204 & 204 \\
\hline Total (kcal) & 105 & 105 \\
\hline Lipid contribution (kcal\%) & $51 \%$ & $51 \%$ \\
\hline
\end{tabular}

* Sum of the water added (in emulsion), and the water in beads

\subsubsection{Study population}

The study population included healthy overweight volunteers (male and female, 18-65 yrs., BMI 25-30 $\mathrm{kg} \cdot \mathrm{m}^{-2}$ ). Thirty-one subjects were needed to complete the study based on the power calculation for differences in food intake, so thirty-three subjects were included to account for possible dropouts. They were recruited from a pool of subjects and via advertisements. Subjects were excluded from participation when they reported milk (-protein or lactose)-allergy/intolerance, dieting, pregnancy, lactation, excessive alcohol consumption (> 20 units per week), intention to stop smoking, self-admitted HIV-positive state, abnormal eating behaviour, or unexplained weight loss/gain in the month prior to screening. Subjects were also excluded when taking medication that may affect appetite and sensory function or who reported metabolic or endocrine disease, GI disorders or a history of medical or surgical events that may have affected study outcome. All included subjects $(n=33$; mean age: 43 yrs.; mean BMI: $27.7 \mathrm{~kg} \cdot \mathrm{m}^{-2}$; 14 male) completed the protocol.

\subsubsection{Study design}

In this randomized single-blind placebo-controlled trial, the effect of two enriched yogurts was compared in a cross-over design, focused on food intake and feelings of satiety. The yogurts differed in the carrier of the lipid: either encapsulated inside alginate beads for ileal delivery (active), or non-encapsulated with similar sensory properties (control). For an overview of the study design, see Figure 7.1. 


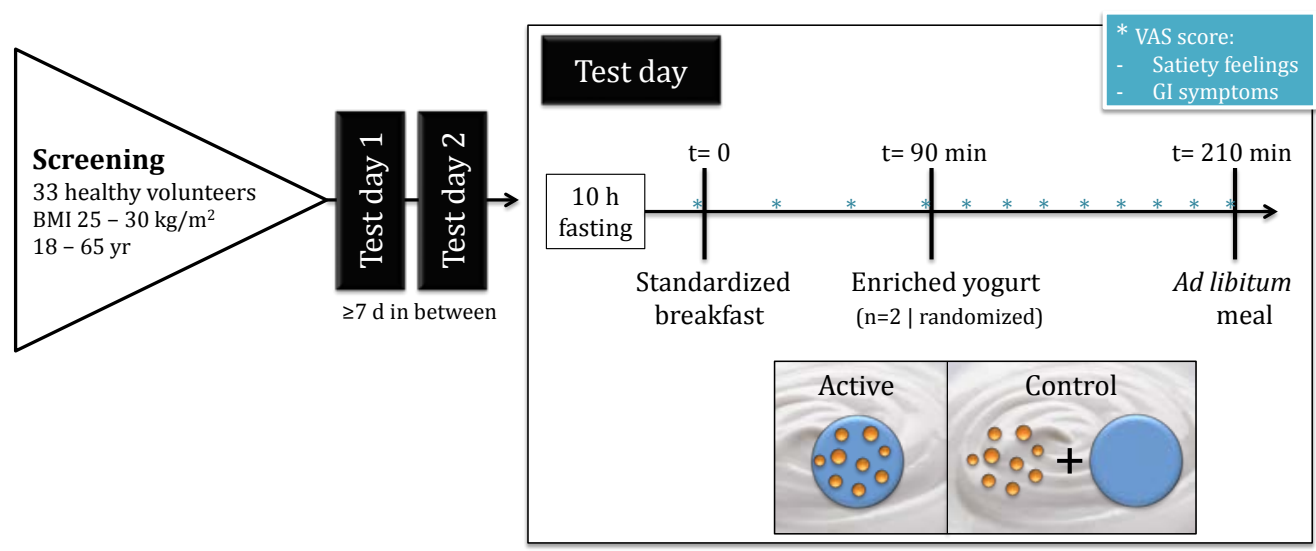

Figure 7.1. Overview of the study design on the effect of encapsulation of lipids on feelings of satiety and food intake. Yogurts were enriched with two types of lipid carrier: either encapsulated as emulsionalginate beads for ileal delivery (active), or non-encapsulated with similar sensory properties (control). * VAS = Visual Analogue Scales, used for nine attributes per time point. GI = gastrointestinal.

\subsubsection{Protocol}

Every subject received two treatments on two different days with at least one week of wash out period, following a randomized cross-over design. On each test day, the subject arrived after a $10 \mathrm{~h}$ fast at the Metabolic Research Unit at the Maastricht University Medical Center+. First, compliance to the previously mentioned rules was checked, and baseline measurements were done for feelings of satiety and gastrointestinal (GI) symptoms. The experiment started with intake of a standardized small breakfast $(\leq 0.5 \mathrm{~g}$ fat; with $150 \mathrm{~mL}$ water or tea; $\mathrm{t}=0$ $\mathrm{min}$ ) that was identical on both test days. When all or most part of the breakfast was expected to have been emptied from the stomach $(t=90 \mathrm{~min})$, the subject received one of the two yogurts (active or control) with $150 \mathrm{~mL}$ water or tea. VAS scores for feelings of satiety and GI symptoms were collected at a 30 min interval before, and a 15 min interval after ingestion of the yogurt. Two hours after ingestion of the yogurt $(t=210 \mathrm{~min})$, food intake was measured, as it reflects the anticipated application of an ileal brake inducing food product, which targets to decrease caloric intake during a subsequent meal. Food intake was measured as the amount eaten (kcal) from a large pasta meal (> $1 \mathrm{~kg}$; Lasagna bolognese, PLUS Supermarket, The Netherlands; energy density per 100 g: 152 kcal, 8.6 g lipid, 11.0 g carbohydrates, $7.1 \mathrm{~g}$ protein). After meal consumption, the test day finished.

\section{Feelings of satiety and GI symptoms}

Visual Analogue Scale (VAS) scores (Parker et al., 2004) were used to determine feelings of satiety (satiety, fullness, hunger, desire to eat, desire to snack) and GI symptoms (bloating, discomfort, pain, nausea), in total at 12 time points ( $\mathrm{t}=0,30,60,90,105,120,135,150,165$, 
180, 195, $210 \mathrm{~min}$ ) per test day. These nine attributes were measured using a VAS from 0 to $100 \mathrm{~mm}$, with the most negative or lowest intensity feelings at the low end and the opposing terms at the high end. The subjects indicated their feeling at that moment; scoring forms were collected immediately to prevent use as reference for later scorings.

\subsubsection{Ethics}

The study was approved by the Medical Ethics Committee of the Maastricht University Medical Center+ and was conducted in full accordance with the principles of the Declaration of Helsinki of 1975 as amended in 2013, and with the Dutch Regulations on Medical Research involving Human Subjects (1998). All participants gave written informed consent before participation. This trial was registered at clinicaltrials.gov as NCT03025997. And was performed at the Maastricht University Medical Center+ from January until March, 2017.

\subsubsection{Statistics}

The SPSS statistical software package Version 23.0.0.2 (IBM@ SPSS Statistics, Chicago, IL, USA) was used for statistical analysis. A descriptive analysis of the study population was performed first, including the independent variables age, gender and BMI, and reported as mean \pm standard error of the mean (SEM).

The dependent variables were checked to meet the requirements homogeneity of variance, and normality was checked and tested (Shapiro-Wilk test, $p>0.05$ ). To compare food intake (kcal) after intake of the control and active yogurt, a paired-samples T-tests was performed $(n=33, p \leq 0.05)$.

Since the raw VAS data did not meet the requirements of normality, we tested the difference between control and active yogurt through area under the VAS curves from ingestion of the yogurt $(\mathrm{t}=90 \mathrm{~min})$ until ad libitum meal consumption $(\mathrm{t}=210 \mathrm{~min})$. The net incremental area under the curves (iAUC, defined as in (Brouns et al., 2005)) of VAS scores were calculated using the trapezoid rule. For feelings of satiety, the effect of yogurt on iAUC VAS scores was tested with a paired-samples T-tests $(n=33, p \leq 0.05)$. For GI symptoms, scores were all very low and the difference in iAUC VAS did not meet normality (Shapiro-Wilk test, $p \leq 0.05$ ), so nonparametric tests for related samples (Wilcoxon Signed Ranks Test, $n=33, p \leq 0.05$ ) were performed.

All data is reported as mean \pm SEM in the results section. 


\subsection{RESULTS}

\subsubsection{Food intake}

The primary goal of the study was to assess the efficacy to reduce food intake through activation of the ileal brake. Figure 7.2 shows the food intake during ad libitum meal consumption, provided $2 \mathrm{~h}$ after ingestion of the active yogurt (black bar; $770 \pm 38 \mathrm{kcal}$ ) or control yogurt (light bar; $821 \pm 40 \mathrm{kcal}$ ). After ingestion of the active yogurt, food intake was significantly lower (51 $\pm 20 \mathrm{kcal} ; p=0.016$ ) compared to the control, which accounts for a reduction of $6.2 \%$ in energy intake.

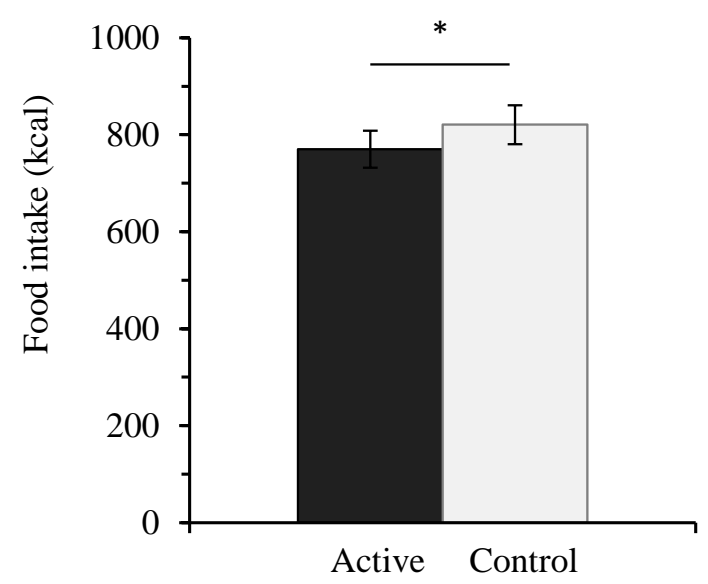

Figure 7.2. Food intake (mean $\pm \mathrm{SEM}$ ) measured during ad libitum meal consumption, $2 \mathrm{~h}$ after ingestion of the active yogurt (black bar) or control yogurt (light bar). The difference is significant in a paired-samples T-test $(* p \leq 0.05)$.

\subsubsection{Feelings of satiety}

The efficacy of the test products to induce feelings of satiety (increased satiety and fullness; reduced hunger, desire to eat and desire to snack) was determined as secondary study parameter, by comparing Visual Analog Scale (VAS) scores. Figure 7.3 shows similar VAS scores for feelings of satiety after ingestion of the active yogurt (filled symbols) and control yogurt (open symbols). Immediately after ingestion of both yogurts, scores for satiety (a) and fullness (b) reached a maximum, followed by a gradual decline, and in contrast, scores for hunger (c), desire to eat (d), and desire to snack (e) reached a minimum followed by a gradual incline. The effect on desire to snack was not as great as on the other factors. 

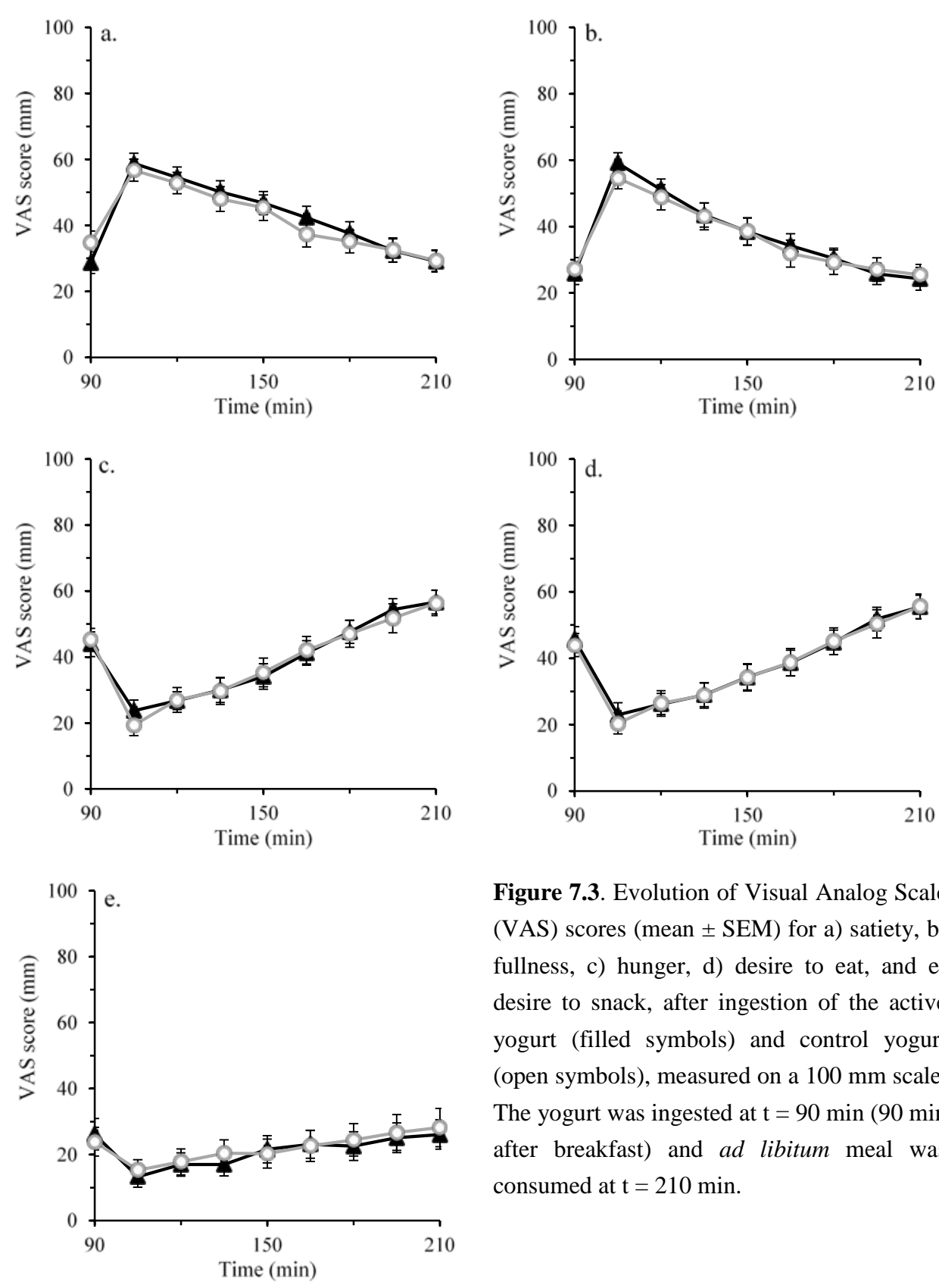

Figure 7.3. Evolution of Visual Analog Scale (VAS) scores (mean \pm SEM) for a) satiety, b) fullness, c) hunger, d) desire to eat, and e) desire to snack, after ingestion of the active yogurt (filled symbols) and control yogurt (open symbols), measured on a $100 \mathrm{~mm}$ scale. The yogurt was ingested at $\mathrm{t}=90 \mathrm{~min}$ (90 $\mathrm{min}$ after breakfast) and ad libitum meal was consumed at $\mathrm{t}=210 \mathrm{~min}$. 
For the encapsulated lipid, Figure 7.4 only shows a higher iAUC VAS for satiety $(+900 \pm$ $344 \mathrm{~mm} \cdot \mathrm{min} ; p=0.013$ ), but this observed increase in satiety did not reach statistical significance after Bonferroni correction for multiple comparisons so we cannot eliminate it to be a Type I error. The iAUC VAS scores for the other satiety attributes were not significantly different between the yogurts (iAUC VAS for hunger, $p=0.6$; for desire to eat, $p=0.7$; for desire to snack, $p=0.3$; for fullness, $p=0.533$ ).

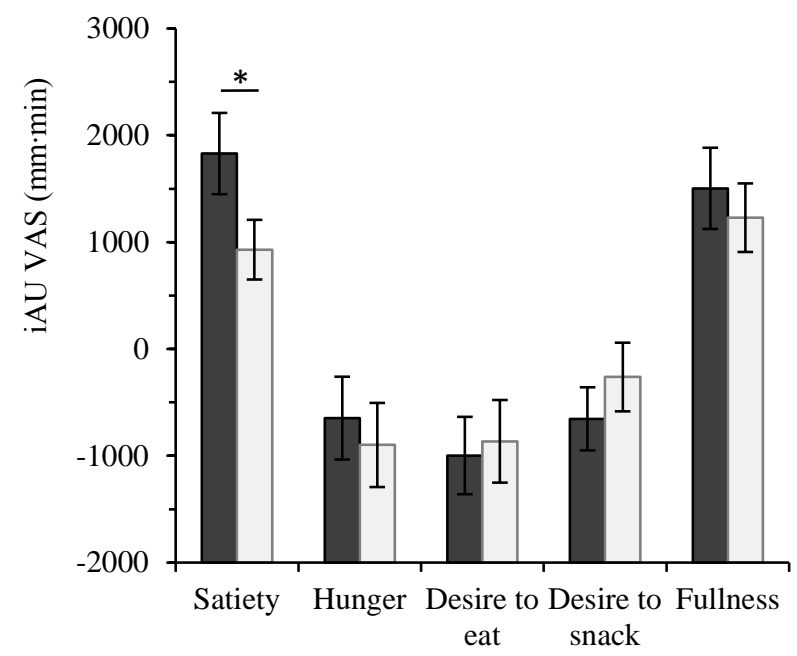

Figure 7.4. Net incremental area under the curve (iAUC, mean \pm SEM) of Visual Analog Scale (VAS) scores from ingestion of the active (black bar) or control (light bar) yogurt ( $\mathrm{t}=90 \mathrm{~min}$ ) until ad libitum meal consumption $(\mathrm{t}=210 \mathrm{~min})$. The difference is significant at $* p \leq 0.05$ in a paired-samples T-test $(n=33)$.

\subsubsection{Gastrointestinal symptoms}

Figure 7.5 shows that all measured gastrointestinal (GI) symptoms were low $(<<10 \mathrm{~mm}$, measured on a $100 \mathrm{~mm}$ scale) and none of the attributes differed significantly in iAUC VAS scores between active and control (Wilcoxon Signed Ranks Test, $n=33, p \leq 0.05$ ). Adverse event evaluation only showed three subjects with short-term diarrhoea: two on the afternoon of the control yogurt and one on the day after the active yogurt. 

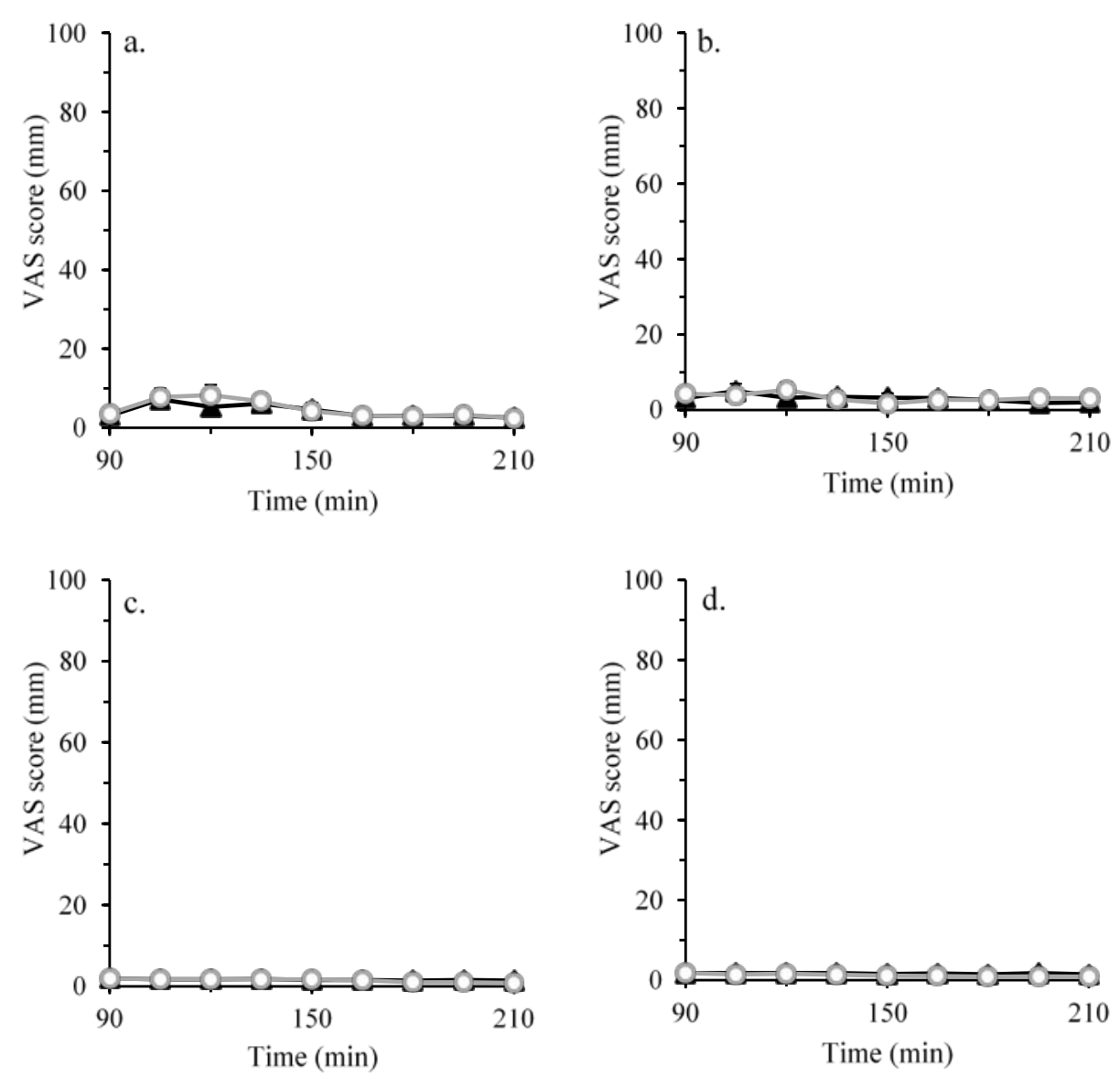

Figure 7.5. Evolution of Visual Analog Scale (VAS) scores (mean \pm SEM) for a) bloating, b) discomfort, c) pain, and d) nausea, after ingestion of the active yogurt (filled symbols) and control yogurt (open symbols), measured on a $100 \mathrm{~mm}$ scale. The yogurt was ingested at $\mathrm{t}=90 \mathrm{~min}$ (90 min after breakfast) and ad libitum meal was consumed at $\mathrm{t}=210 \mathrm{~min}$.

\subsection{DISCUSSION}

We have shown that ingestion of a yogurt containing emulsion-alginate beads results in a significant reduction in food intake during a subsequent meal ( $-6.2 \%$ energy intake) compared to the control yogurt that contained an equicaloric mixture of non-encapsulated nutrients with similar sensory properties. To the best of our knowledge, this is the first human intervention study exploring the effects of emulsion-alginate beads on food intake and feelings of satiety. Food intake was reduced without increasing GI symptoms. Satiety was significantly increased after intake of the active yogurt compared to the control ( $p=0.013$ ), but fullness, hunger, desire to eat, and desire to snack were not different between the interventions. The observed increase in satiety did not reach statistical significance after 
Bonferroni correction for multiple comparisons. Thus, while emulsion-alginate beads clearly affect eating behaviour, no significant changes in feelings of satiety or other subjective feelings that are associated with food intake, were observed.

Reduced food intake through activation of the ileal brake has previously been proven with direct intra-ileal infusion of lipid (Maljaars et al., 2011; van Avesaat et al., 2015; Welch et al., 1985) but not after oral ingestion. Oral ingestion of Fabuless ${ }^{\circledR}$, also named Olibra ${ }^{\circledR}$ and Reducal $^{\circledR}$, increases the amount of lipid degradation products in the jejunum (Knutson, Fridblom, Viberg, Sein, \& Lennerna, 2010); and although Fabuless ${ }^{\circledR}$ loses its functionality once subjected to normal food-manufacturing processes (thermal and shear processing), it had only a weak effect on food intake but no effect on appetite (2011) and improved weight management when unprocessed (Diepvens, Soenen, Steijns, Arnold, \& WesterterpPlantenga, 2007). Compared to intubation studies that deliver exact amounts of lipid directly to the target position (Alleleyn et al., 2016), the effect that we find here is less pronounced. This may be related to less optimal timing and dosages of the release of lipids. Secondly, lean subjects were used in the intubation studies while we studied overweight subjects, which are known to be less sensitive for intraluminal lipids (Stewart et al., 2011). We anticipate that part of the encapsulated lipid will have remained unabsorbed as discussed below. Further optimization of the emulsion-alginate beads should be attempted.

Ad libitum food intake was assessed $2 \mathrm{~h}$ after intake of the test yogurts, as this reflects the anticipated application of an ileal brake inducing food product, which targets to decrease caloric intake during a subsequent meal. Within this time scale, we observed no significant effect of the yogurt type on scores of satiety. Based on the average gastric emptying profile of yogurt, about half of the emulsion-alginate beads was expected to reach the small intestine within 45 min after ingestion (Berrada, Lemeland, Laroche, Thouvenot, \& Piaia, 1991) and once in the small intestine, half of the lipid digestion products were expected to be released within 2.5 h based on previous observations in a static in vitro model (Corstens, BertonCarabin, Elichiry-Ortiz, et al., 2017). Based on the in vitro observations, it is likely that only a part of the encapsulated lipids will have been digested before subsequent meal consumption started. This may explain why both interventions did not show differences in feelings of satiety. Based on our findings we expect the feelings of satiety to be more affected on a longer time scale, especially because the beads are expected to have an extended residence time in the ileum (Hoad et al., 2009). In addition to study design (timing and dosage), the bead design needs optimization to further increase the potential to target in vivo delivery of encapsulated lipid into the ileum. This can be done by making the encapsulated lipid more accessible for lipase through tailoring of the size of the beads and/or the mesh size as described previously (Corstens, Berton-Carabin, Elichiry-Ortiz, et al., 2017). Increasing the amount of encapsulated lipid per bead volume is not considered to be a target for further optimization, 
as the resulting bead density would most likely result in floating of the beads in the stomach and, thus, delaying emptying of the active components into the small intestine.

The control yogurt is considered an appropriate reference for the active yogurt as the free emulsion is rapidly digested and the lipid metabolites will probably be absorbed completely before reaching the distal small intestine (Camilleri \& Sweetser, 2007; Little et al., 2007; Van Citters \& Lin, 1999). Moreover, the empty beads can conveniently be used to design equicaloric products that have no difference in mouth feel as none of the subjects indicated a difference in sensory properties between the two yogurts.

A shortcoming of the present study is that the distal release of lipids from the used encapsulates was not tested during the in vivo experiment but instead, the digestion dynamics of the encapsulated lipids were anticipated, based on in vitro observations. Additional mechanistic studies are now required to measure delivery of lipids from ingested emulsionalginate beads in vivo in the intestinal lumen.

To gain further understanding of ileal brake activation for weight management, additional studies need to be conducted. Regarding the short-term single-dose effect, the duration and impact of the effect will have to be studied in more detail and on a longer time scale, e.g., determine optimal timing of ingestion of the test product in relation to meal consumption, and studying the effects of the encapsulated lipids over a full day, as beads are expected to have an extended residence time in the ileum (Hoad et al., 2009). The use of biological markers (i.e., gut hormones as PYY and GLP-1) will contribute to further understanding of satiating mechanisms, enabling further development of the treatment. In addition, the bead and yogurt composition needs optimization based on dose-response studies, which may even lead to personalization of the beads to specific effects that occur within individuals. In a next phase, the application of the beads to modulate food intake should be studied in long-term intervention studies, investigating effects of this concept on body weight regulation, while also investigating possible habituation and compensation. These assumptions and hypotheses will have to be further explored in the near future.

The future perspectives of emulsion-alginate beads for non-invasive weight management are very promising, because these beads can easily be incorporated into a food product that is part of a normal diet as demonstrated here in a yogurt. It is also good to mention that there are positive developments in production technology, to produce similar emulsions and beads at a large scale (Schroën, Bliznyuk, Muijlwijk, Sahin, \& Berton-Carabin, 2015).

In conclusion, we demonstrated that ingested emulsion-alginate beads significantly decreased food intake in overweight individuals compared to an equicaloric mixture of nonencapsulated nutrients with similar sensory properties. We hypothesize that this reduction in food intake is caused by release of encapsulated lipids in the distal small intestine, which 
activates the ileal brake mechanism. To gain further understanding of ileal brake activation via dietary routes for weight management, additional studies on dose-response relations, and long-term effectiveness need to be conducted. If successful, this strategy will result in noninvasive methods for weight control.

\section{Acknowledgements}

Research presented in this publication was financially supported by the Graduate School VLAG. We thank Gabby Hul for her valuable assistance with the logistics in the Metabolic Research Unit Maastricht. The authors' responsibilities were as follows-MNC, AMEA, FJT, and AAMM designed the research; MNC, FJT, and AAMM analysed the data, and had primary responsibility for the final content of the manuscript; MNC and TK conducted the research; MNC performed the statistical analysis; MNC, FJT, KS, CCBC, and AAMM wrote the manuscript; and all authors read, commented and approved the final manuscript. None of the authors reported a conflict of interest related to the study.

\subsection{REFERENCES}

Alleleyn, A. M. E., van Avesaat, M., Troost, F. J., \& Masclee, A. A. M. (2016). Gastrointestinal Nutrient Infusion Site and Eating Behavior: Evidence for A Proximal to Distal Gradient within the Small Intestine? Nutrients, 8(3), 117-132.

Bakala N’Goma, J.-C., Amara, S., Dridi, K., Jannin, V., \& Carrière, F. (2012). Understanding the lipiddigestion processes in the GI tract before designing lipid-based drug-delivery systems. Therapeutic Delivery, 3(1), 105-124.

Berrada, N., Lemeland, J. F., Laroche, G., Thouvenot, P., \& Piaia, M. (1991). Bifidobacterium from fermented milks: survival during gastric transit. Journal of Dairy Science, 74(2), 409-413.

Brouns, F., Bjorck, I., Frayn, K. N., Gibbs, A. L., Lang, V., Slama, G., \& Wolever, T. M. S. (2005). Glycaemic index methodology. Nutrition Research Re, 18, 145-171.

Camilleri, M., \& Sweetser, S. (2007). Would free fatty acids enhance treatment of obesity? Gastroenterology, 133(4), 1367-1370.

Corstens, M. N., Berton-Carabin, C. C., de Vries, R., Troost, F. J., Masclee, A. A. M., \& Schroën, K. (2017). Food-grade micro-encapsulation systems that may induce satiety via delayed lipolysis: A Review. Critical Reviews in Food Science and Nutrition, 57(10), 2218-2244.

Corstens, M. N., Berton-carabin, C. C., Elichiry-Ortiz, P. T., Hol, K., Troost, F. J., Masclee, A. A. M., \& Schroën, K. (2017). Emulsion-alginate beads designed to control in vitro intestinal lipolysis: Towards appetite-control. Journal of Functional Foods, 34(July), 319-328.

Corstens, M. N., Berton-Carabin, C. C., Kester, A., Fokkink, R., van den Broek, J. M., de Vries, R., ... Schroën, K. (2017). Destabilization of multilayered interfaces in digestive conditions limits their ability to prevent lipolysis in emulsions. Food Structure, 12(April), 54-63.

Corstens, M. N., Berton-Carabin, C. C., Schroën, K., Troost, F. J., Masclee, A. A. M., Viau, M., \& Meynier, A. (2017). Emulsion encapsulation in alginate beads retards lipolysis during dynamic in vitro digestion. In Poster at the 5th International Conference on Food Digestion; Rennes, France.

Corstens, M. N., Osorio Caltenco, L. A., de Vries, R., Schroën, K., \& Berton-Carabin, C. C. (2017). Interfacial behaviour of biopolymer multilayers: Influence of in vitro digestive conditions. Colloids and Surfaces B: Biointerfaces, 153(May), 199-207.

Diepvens, K., Soenen, S., Steijns, J., Arnold, M., \& Westerterp-Plantenga, M. (2007). Long-term effects of consumption of a novel fat emulsion in relation to body-weight management. International Journal of Obesity, 31(6), 942-9.

Hoad, C., Rayment, P., Cox, E., Wright, P., Butler, M., Spiller, R., \& Gowland, P. (2009). Investigation of alginate beads for gastro-intestinal functionality, Part 2: In vivo characterisation. Food Hydrocolloids, 23(3), 833-839. 
Hoad, C., Rayment, P., Risse, V., Cox, E., Ciampi, E., Pregent, S., ... Gowland, P. (2011). Encapsulation of lipid by alginate beads reduces bio-accessibility: An in vivo 13C breath test and MRI study. Food Hydrocolloids, 25(5), 1190-1200.

Knutson, L., Fridblom, H., Viberg, A., Sein, A., \& Lennerna, H. (2010). Gastrointestinal metabolism of a vegetable-oil emulsion in healthy. American Journal of Clinical Nutrition, 92, 515-524.

Li, Y., Hu, M., Du, Y., Xiao, H., \& McClements, D. J. (2011). Control of lipase digestibility of emulsified lipids by encapsulation within calcium alginate beads. Food Hydrocolloids, 25(1), 122-130.

Li, Y., Kim, J., Park, Y., \& McClements, D. J. (2012). Modulation of lipid digestibility using structured emulsion-based delivery systems: comparison of in vivo and in vitro measurements. Food \& Function, 3(5), 528-36.

Little, T. J., Russo, A., Meyer, J. H., Horowitz, M., Smyth, D. R., Bellon, M., ... Feinle-Bisset, C. (2007). Free fatty acids have more potent effects on gastric emptying, gut hormones, and appetite than triacylglycerides. Gastroenterology, 133(4), 1124-31.

Maljaars, P. W. J., Peters, H. P. F., Kodde, A., Geraedts, M., Troost, F. J., Haddeman, E., \& Masclee, A. A. M. (2011). Length and site of the small intestine exposed to fat influences hunger and food intake. The British Journal of Nutrition, 106(10), 1609-15.

Maljaars, P. W. J., Peters, H. P. F., Mela, D. J., \& Masclee, A. A. M. (2008). Ileal brake: a sensible food target for appetite control. A review. Physiology \& Behavior, 95(3), 271-281.

Maljaars, P. W. J., Romeyn, E. A., Haddeman, E., Peters, H. P. F., \& Masclee, A. A. M. (2009). Effect of fat saturation on satiety, hormone release, and food intake. American Journal of Clinical Nutrition, 89, 1019-1024.

Parker, B. A., Sturm, K., MacIntosh, C. G., Feinle, C., Horowitz, M., \& Chapman, I. M. (2004). Relation between food intake and visual analogue scale ratings of appetite and other sensations in healthy older and young subjects. European Journal of Clinical Nutrition, 58(2), 212-218.

Rayment, P., Wright, P., Hoad, C., Ciampi, E., Haydock, D., Gowland, P., \& Butler, M. F. (2009). Investigation of alginate beads for gastro-intestinal functionality, Part 1: In vitro characterisation. Food Hydrocolloids, 23(3), 816-822.

Schroën, K., Bliznyuk, O., Muijlwijk, K., Sahin, S., \& Berton-Carabin, C. C. (2015). Microfluidic emulsification devices: from micrometer insights to large-scale food emulsion production. Current Opinion in Food Science, 3, 33-40.

Smit, H. J., Keenan, E., Kovacs, E. M. R., Wiseman, S. a, Peters, H. P. F., Mela, D. J., \& Rogers, P. J. (2011). No efficacy of processed Fabuless (Olibra) in suppressing appetite or food intake. European Journal of Clinical Nutrition, 65(1), 81-6.

Stewart, J. E., Seimon, R. V., Otto, B., Keast, R. S. J., Clifton, P. M., \& Feinle-Bisset, C. (2011). Marked differences in gustatory and gastrointestinal sensitivity to oleic acid between lean and obese men. American Journal of Clinical Nutrition, 93(4), 703-711.

van Avesaat, M., Troost, F. J., Ripken, D., Hendriks, H. F., \& Masclee, A. A. M. (2015). Ileal brake activation: macronutrient specific effects on eating behavior? International Journal of Obesity, 39(April), 235243.

Van Citters, G. W., \& Lin, H. C. (1999). The ileal brake: a fifteen-year progress report. Current Gastroenterology Reports, 1(5), 404-409.

Welch, I., Saunders, K., \& Read, N. W. (1985). Effect of ileal and intravenous infusions of fat emulsions on feeding and satiety in human volunteers. Gastroenterology, 89(6), 1293-1307.

Welch, I., Sepple, C. P., \& Read, N. W. (1988). Comparisons of the effects on satiety and eating behaviour of infusion of lipid into the different regions of the small intestine. Gut, 29, 306-311.

Wright, P. J., Ciampi, E., Hoad, C. L., Weaver, A. C., van Ginkel, M., Marciani, L., ... Rayment, P. (2009). Investigation of alginate gel inhomogeneity in simulated gastro-intestinal conditions using magnetic resonance imaging and transmission electron microscopy. Carbohydrate Polymers, 77(2), 306-315.

Zeeb, B., Saberi, A. H., Weiss, J., \& McClements, D. J. (2015). Retention and release of oil-in-water emulsions from filled hydrogel beads composed of calcium alginate: impact of emulsifier type and $\mathrm{pH}$. Soft Matter, 11, 2228-2236. 


\section{Chapter 8}

General discussion 


\subsection{Introduction}

In order to activate the ileal brake mechanism and induce feelings of satiety, lipids and its digestion products need to be delivered to the ileum. From the findings in this thesis it is clear that this is very challenging due to the efficient digestive processes in the gastrointestinal (GI) tract that result in absorption already in the proximal small intestine. For ileal delivery, the digestive breakdown of dietary lipids need to be delayed, and this may be achieved by encapsulating the nutritional lipids.

As a starting point for our investigations, we aimed at encapsulation systems for safflower oil, which is known to give the strongest ileal brake when infused into the ileum through intubation. The approach was multidimensional, going from the physical stability of the encapsulation systems (both multilayered emulsions, and emulsion-alginate beads), to their behaviour during in vitro digestion (static and dynamic models), and eventually towards a clinical trial to explore the effect on ad libitum food intake and satiety. This chapter starts with a discussion of the main findings of this thesis, including a reflection on the methods to study digestion, incorporation of the systems in food, and relevance for weight management. The chapter concludes with an outlook on the steps that need to be taken for implementation, including technical feasibility.

\subsection{Discussion of main findings}

In order to release lipolysis products into the distal small intestine, two strategies were considered: oil-water interfacial design in emulsions and emulsion entrapment in hydrogel beads. Through oil-water interfacial design in emulsions, lipase adsorption could be delayed, and thus also digestion. Alternatively, emulsion droplets could be protected by entrapment in hydrogel beads to delay diffusion of lipase, making the oil-water interface less accessible. Initially, both approaches were considered, but the data presented in this thesis lead us to conclude that delaying the action of lipase with emulsion-alginate beads is much more effective than doing so with multilayered and structured interfaces.

\subsubsection{Main findings on interfacial design}

A lot of research has been done on the relation between the interfacial structure of emulsions and lipolysis, as discussed in Chapter 2, amongst which multilayered interfaces. Initially, we considered these as potentially protective, especially when at least one of the components was of indigestible nature. However, the findings shown in Chapter 3, in which we studied emulsion systems during in vitro GI digestion, and Chapter 4, in which we looked at the behaviour of model interfaces under similar conditions, point out that this approach is not effective. Additional layers may have increased the adsorbed amount in multilayered interfaces, leading to a mesoscopically heterogeneous structure, but during in vitro intestinal 
digestion, this did not influence lipolysis that much. When harsher digestive conditions were simulated (representing the physiological fed state) the effect on lipolysis disappeared completely. The multilayers destabilised rapidly at neutral intestinal $\mathrm{pH}$, as observed with interfacial tension and rheology experiments, allowing bile and/or lipase to efficiently adsorb, leading to extensive and fast digestion. These findings lead us to conclude that lipolysis cannot be controlled by designing a protective interfacial structure in emulsions.

\subsubsection{Main findings on emulsion-alginate beads}

As an alternative approach, emulsion-hydrogel beads made of indigestible alginate crosslinked with calcium ions were applied (Chapters 5-7). Chapter 5 describes the design of such beads in terms of bead and mesh size, and their behaviour in static in vitro GI conditions. Emulsion-alginate bead volume was $\mathrm{pH}$-dependent, which was purposely used for targeted release in the distal small intestine. This was due to a slight decrease in bead volume at acidic $\mathrm{pH}$, which mimics stomach transit, therewith reducing the mesh size and limiting lipase diffusion, whereas at $\mathrm{pH} 7.0$, as is observed in the distal parts of the small intestine, the bead volume increased, leading to larger mesh size, and facilitated lipase diffusion.

When tested in static in vitro GI digestion experiments (Chapter 5), the emulsion-alginate beads showed a very different digestion profile compared to free emulsion droplets that are easily digested. Moreover, there was a notable difference between the emulsion-alginate beads depending on bead and mesh size, for which a descriptive model was developed. Chapter 6 relates to dynamic in vitro GI digestion, confirming the great differences between the digestion profile of emulsion-alginate beads and free emulsion droplets. The obtained data showed that emulsion-alginate beads postponed the release of free fatty acid (FFA) for about $3 \mathrm{~h}$, making them relevant for release in the distal small intestinal. Therefore, emulsion-alginate beads were used as active ingredient in a placebo-controlled trial with cross-over design to study the effect on food intake and feelings of satiety in overweight individuals (Chapter 7). The ingestion of a yogurt containing emulsion-alginate beads had a significant, albeit small, effect on food intake during the subsequent meal (-6.2\%) compared to the control condition (yogurt with an equicaloric mixture of non-encapsulated nutrients, having similar sensory properties).

\subsubsection{Comparison in vitro-in vivo experiments}

When comparing the outcomes of the in vitro to the in vivo experiments, it is clear that in vitro experiments are much cheaper, faster and easier to implement and perform, which makes them very suitable as a screening tool (see also Table $\mathbf{8 . 1}$ for a comparison of arguments). In general, it should be mentioned that it is unclear to what extent in vitro lipid digestion gives an accurate representation of the in vivo situation due to the absence of 
complementary in vitro-in vivo markers and a limited gastric phase (Bohn et al., 2017; Carrière, 2016). Another big difference between the in vivo situation and the most used static method for in vitro lipid digestion (i.e., pH stat), is that digestion products are not removed from the digestive media, which could largely affect enzymatic reactions. Besides, the outcome in an in vitro assay depends on the chosen and varying parameters, such as $\mathrm{pH}$, calcium, and type and concentration of enzymes (Li, Hu, \& McClements, 2011; Sams, Paume, Giallo, \& Carrière, 2016). In our study, in vitro experiments brought more insight in the release behaviour compared to the in vivo experiment. The clinical in vivo trial provided evidence for the effectiveness of the ileal brake in food intake regulation, and justifies followup research.

Table 8.1. Comparison between the performed experiments to study digestion in this thesis.

\begin{tabular}{lccccc}
\hline \multicolumn{1}{c}{ Experiment type } & Costs & Rapidness & $\begin{array}{c}\text { Ease to } \\
\text { implement }\end{array}$ & $\begin{array}{c}\text { Ease to } \\
\text { perform }\end{array}$ & $\begin{array}{c}\text { Close to real } \\
\text { conditions }\end{array}$ \\
\hline Static in vitro & ++ & ++ & ++ & + & - \\
Dynamic in vitro & + & + & $+/-$ & $+/-$ & $+/-$ \\
In vivo & - & - & - & - & ++ \\
\hline An & & & &
\end{tabular}

An advantage (+), main advantage (++), drawback (-).

\subsubsection{Relevance for weight management}

Direct intra-ileal infusion of lipids has been shown to activate the ileal brake and to reduce food intake with 15 to 32\% compared to control (saline infusion), but this is an invasive method (Maljaars et al., 2011; van Avesaat, Troost, Ripken, Hendriks, \& Masclee, 2015; Welch, Saunders, \& Read, 1985; Welch, Sepple, \& Read, 1988). One oral product has been commercialized to increase the amount of lipid degradation products in the jejunum, and is known under the brands Fabuless ${ }^{\circledR}$, Olibra ${ }^{\circledR}$ and Reducal ${ }^{\circledR}$ (Knutson, Fridblom, Viberg, Sein, \& Lennerna, 2010). When not exposed to food-manufacturing processes, this product is capable of reducing food intake (Burns, Livingstone, Welch, Dunne, \& Rowland, 2002; Smit et al., 2011) and improved weight management (Diepvens, Soenen, Steijns, Arnold, \& Westerterp-Plantenga, 2007); however, it loses functionality once subjected to classic thermal and shear processing (Smit et al., 2011).

Compared to intubation studies, the satiating effect after oral application is less pronounced, which is most likely related to the direct delivery of exact amounts of lipid at the target position when using intubation, whereas oral applications results in less optimal timing and dosing of the lipids. Furthermore, it should be taken into account that in the intubation studies lean subjects were considered whereas we studied overweight subjects, which are known to be less sensitive for intraluminal lipids (Stewart et al., 2011). 
Emulsion-alginate beads have not been studied before in humans for their effects on food intake and feelings of satiety. They reduced food intake during the subsequent meal $(-6.2 \%)$ compared to control, which is of high relevance for weight management. When extrapolating these findings directly to a daily basis, a reduction of $155 \mathrm{kcal}$ could be achieved for a 2,500 kcal intake. On annual basis, such a daily reduction in kcal intake could have important implications for weight management. Whether this can also be achieved in daily practice, highly depends on individual compliance to the weight management strategy, and indirectly also on the social environment (WHO, 2017).

The use of ileal brake activation is a very different approach compared to conventional strategies to lose weight, such as (very) low calorie diets. The origin of the reduction in calorie intake is different, being via a natural feedback-mechanism in the ileal brake approach but imposed in conventional strategies. Besides, the daily reduction in calorie intake is lower in the ileal brake approach, so weight loss will be more gradual compared to conventional strategies. Therefore, the ileal brake approach will be less susceptible to the well-known weight cycling (i.e., yo-yo effect) compared to conventional strategies, so weight loss is better maintained on a long-term.

\subsection{Outlook}

It is clear that emulsion-alginate beads hold promises for use in weight management strategies. This section will discuss the most relevant aspects to implement this product: physiological effectiveness (e.g., understanding the mechanism of action, dose-response relations and long-term effectiveness), technical optimization of the beads, and incorporation in food and large-scale production.

\subsubsection{Physiological effectiveness}

The distal release of lipids from emulsion-alginate beads was measured by in vitro observations. To understand the underlying mechanisms, distal release of lipids needs to be measured in vivo through sampling from the intestinal lumen. The most important factors to look at will be biological markers (i.e., gut hormones such as Peptide YY) and gastric emptying rates. In this respect, it is essential to establish dose-response relations between the quantity of sensed lipids and the effectiveness of triggering the ileal brake, as assessed primarily by food intake regulation. Based on that, the optimal timing of ingestion of the test product in relation to meal consumption needs to be determined.

Emulsion-alginate beads are expected to induce feelings of satiety that last longer than only the tested $2 \mathrm{~h}$ in Chapter 7, not only because the beads are expected to have an extended release profile and an extended residence time in the ileum, but also because the satiety hormones cause a continuation of the ileal brake effect. In follow up research, the effect of 
ingested emulsion-alginate beads should be studied over a full day, after a single ingestion or testing multiple ingestions spread over that day. Next, a long-term intervention study, e.g., several weeks or even months, should be carried out to investigate the effect on bodyweight regulation, while also investigating possible habituation to and compensation of the observed food intake inhibition.

\subsubsection{Bead design}

In order to let the beads pass the stomach they preferably should not float on top, which limits the amount of oil that can be encapsulated (due to the low density of oil). In contrast, gradual gastric release (e.g., of stomach specific drugs) would benefit from floating beads $(\geq 10 \%$ vegetable oil induced floating in water, physiological saline and $\mathrm{HCl}$ solution that mimics gastric juice) (Murata, Sasaki, Miyamoto, \& Kawashima, 2000). To improve dosing, the release behaviour can be tuned through adjusting the bead size, the mesh size of the beads, and the size of the emulsion droplets in the beads (Figure 8.1).

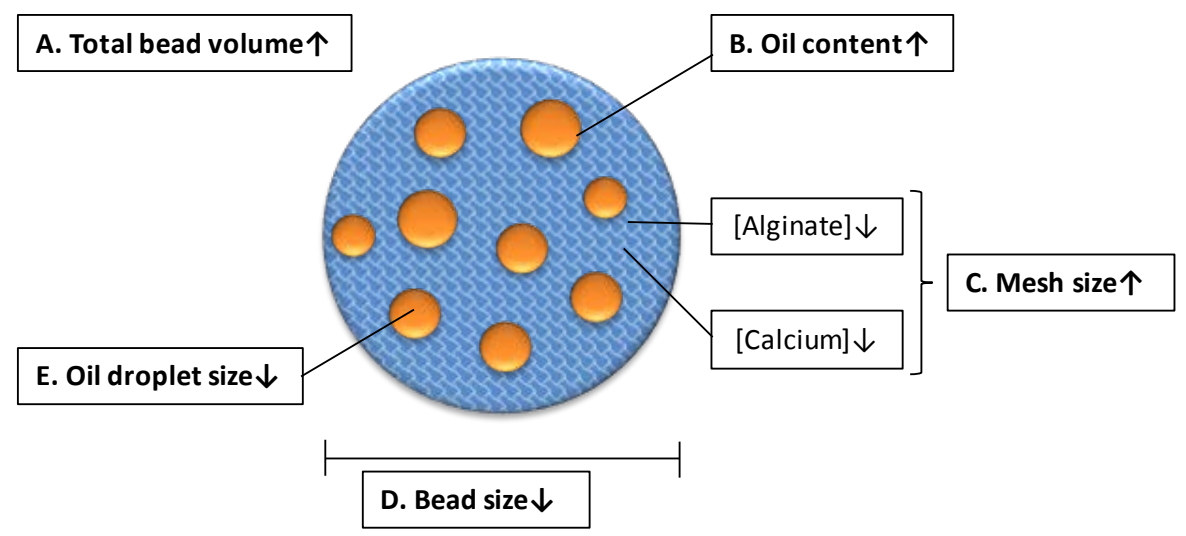

Figure 8.1. Schematic overview of factors to increase $(\uparrow)$ or decrease $(\downarrow)$ in order to increase the sensed lipid dosage. 
To maximize the ileal brake effect, the highest amounts of metabolites must be released and sensed in the small intestine during transit of the beads in the ileum. For this the model developed in Chapter 5 can be used, for example to reduce meal consumption $2 \mathrm{~h}$ after bead ingestion as shown in Figure 8.3 (and done in Chapter 7). The model can also be used to investigate the effect of emulsion droplet size by increasing the interface dependent reaction rate (as shown in Figure 8.2), as experimentally confirmed by others (Cho et al., 2014; Lett, Norton, \& Yeomans, 2016; Maljaars et al., 2012). In Figure 8.2, the effect of a reduction in the droplet size (by a factor of 2, 5 and 10) is shown. It is clear that droplet size has a great effect on the release profile, and is an important extra parameter to control lipolysis.

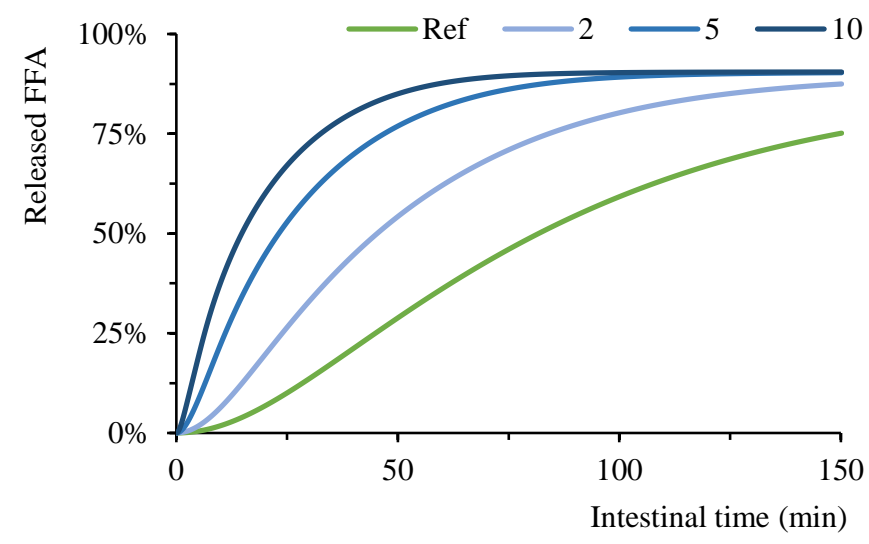

Figure 8.2. Effect of reduced emulsion droplet size in emulsion-alginate beads on released free fatty acid (FFA) during intestinal incubation, based on the developed model in Chapter 5 and a bead size of $0.6 \mathrm{~mm}$ and mesh size of $8 \mathrm{~nm}$. The number indicates the reduction factor of the droplet size, with as reference (Ref) an emulsion droplet size of $21 \mu \mathrm{m}$. 
The model could even forecast a personalized bead, if the desired release pattern is known. An example of how the model could be used is illustrated in design diagrams that show the overall predicted FFA release as a function of diffusion distance (based on bead size) and effective diffusion coefficient (based on mesh size). These design diagrams can be used to not only target a specific time-release profile (e.g., Supplementary Figure 5.10), but related to that to reduced meal consumption, e.g., Figure 8.3 in which satiety induction, $2 \mathrm{~h}$ after bead ingestion is targeted, as was the case in Chapter 7. When assuming the relevant time frame for sensing in the ileum to be between 90 and 120 min after bead ingestion, and an average gastric emptying time of 45 min (Berrada, Lemeland, Laroche, Thouvenot, \& Piaia, 1991), the corresponding intestinal time to reach the ileum would be between 45 and 75 min.

Figure 8.3 shows the sum of released FFA in this intestinal time frame for a range of bead designs that differ in bead and mesh size. It is clear that various combinations can be used to reach the same release.

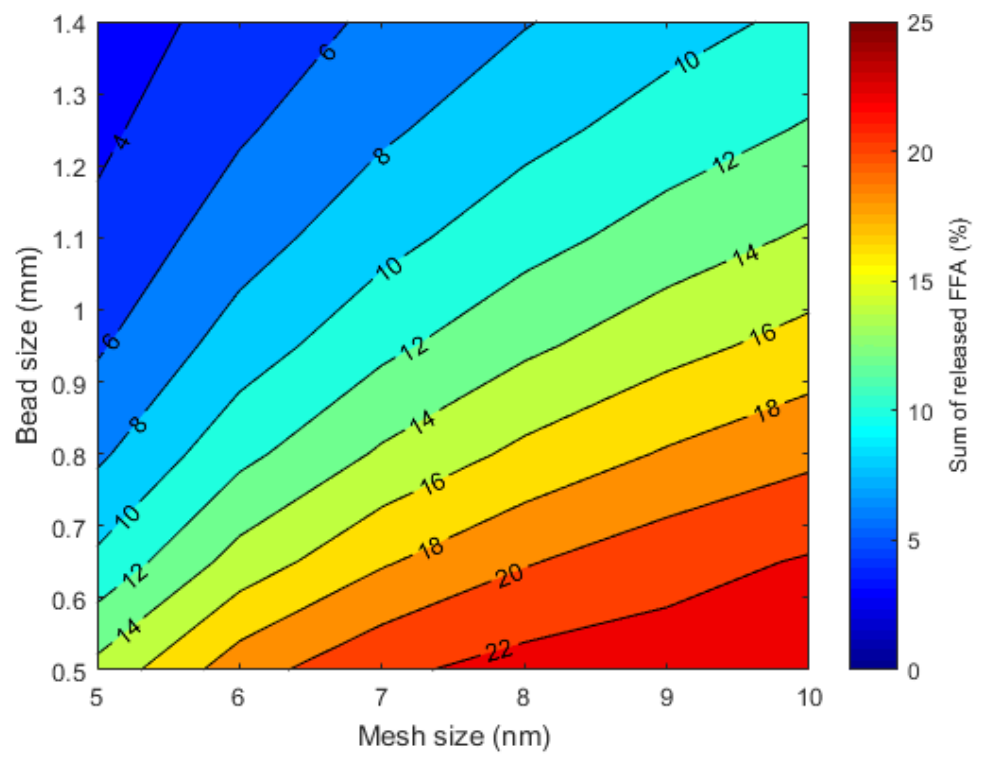

Figure 8.3. Predicted total release of free fatty acids (FFA) (\%) from emulsion-alginate gel beads that is expected to be sensed in the distal small intestine, between 45 and 75 min intestinal incubation, as function of bead and mesh size.

\subsubsection{Incorporation of emulsion-alginate beads in food}

For incorporation in food, the size of the beads is important. Ideally they should not affect the sensory properties. In that respect, emulsion droplets may be much more easily incorporated in a food product without being noticed (but they would be digested much too rapidly when not protected). Although the sensory properties will change using the current size of emulsion-alginate beads, they can easily be incorporated into a food as demonstrated 
for yogurt, and will enter the small intestine almost unchanged. Please note that the functionality of emulsion-alginate beads once subjected to classic thermal- and shearprocessing needs to be studied as well, as it can result is loss of functionality as known for Fabuless $^{\circledR}$ (Smit et al., 2011).

\subsubsection{Large-scale bead production}

In order to commercialize a food containing emulsion-alginate beads, the production technology needs further development to increase the production rate. One could think of a batch process, for example the commercially available Encapsulator B-395 (Büchi) that can produce sterile beads at rates up to $200 \mathrm{~mL} / \mathrm{min}$ according to specifications given by the supplier, but to reach a true scale-up, productivity would need to be improved.

In order to achieve this, the current dispersion method could be improved as the liquid-air dripping method has a limited drop-by-drop production rate (Leong et al., 2016). Alternative liquid-air dispersion methods that have a higher production rate are jetting (i.e., breakup or cutting of a laminar liquid jet) and atomization (i.e., dispersion into aerosols that are subsequently gelled) (Leong et al., 2016), but liquid-liquid dispersion method could be preferred. This might be achieved using micro-/milli-fluidic devices, resulting in highly monodispersed and specific particles as shown for oil-core alginate-shell particles (Martins, Poncelet, Marquis, Davy, \& Renard, 2017) and even Janus hydrogels (Marquis, Davy, Cathala, Fang, \& Renard, 2015). Although such devices are generally known to have a low throughput, there are positive developments in their upscaling (Schroën, Bliznyuk, Muijlwijk, Sahin, \& Berton-Carabin, 2015). This may lead to large-scale bead production and commercialization of the resulting product, which ultimately can be used in a noninvasive weight management strategy to reduce the global obesity endemic.

\subsection{References}

Berrada, N., Lemeland, J. F., Laroche, G., Thouvenot, P., \& Piaia, M. (1991). Bifidobacterium from fermented milks: survival during gastric transit. Journal of Dairy Science, 74(2), 409-413.

Bohn, T., Carriere, F., Day, L., Deglaire, A., Egger, L., Freitas, D., ... Mackie, A. R. (2017). Correlation between in vitro and in vivo data on food digestion. What can we predict with static in vitro digestion models? Critical Reviews in Food Science and Nutrition.

Burns, A., Livingstone, M., Welch, R., Dunne, A., \& Rowland, I. (2002). Dose-response effects of a novel fat emulsion $\left(\mathrm{Olibra}^{\mathrm{TM}}\right)$ on energy and macronutrient intakes up to $36 \mathrm{~h}$ post-consumption. European Journal of Clinical Nutrition, 56(4), 368-377.

Carrière, F. (2016). Impact of gastrointestinal lipolysis on oral lipid-based formulations and bioavailability of lipophilic drugs. Biochimie, 125, 297-305.

Cho, H. T., Salvia-Trujillo, L., Kim, J., Park, Y., Xiao, H., \& McClements, D. J. (2014). Droplet size and composition of nutraceutical nanoemulsions influences bioavailability of long chain fatty acids and Coenzyme Q10. Food Chemistry, 156, 117-22.

Diepvens, K., Soenen, S., Steijns, J., Arnold, M., \& Westerterp-Plantenga, M. (2007). Long-term effects of consumption of a novel fat emulsion in relation to body-weight management. International Journal of Obesity, 31(6), 942-9. 
Knutson, L., Fridblom, H., Viberg, A., Sein, A., \& Lennerna, H. (2010). Gastrointestinal metabolism of a vegetable-oil emulsion in healthy. American Journal of Clinical Nutrition, 92, 515-524.

Leong, J. Y., Lam, W. H., Ho, K. W., Voo, W. P., Lee, M. F. X., Lim, H. P., ... Chan, E. S. (2016). Advances in fabricating spherical alginate hydrogels with controlled particle designs by ionotropic gelation as encapsulation systems. Particuology, 24, 44-60.

Lett, A. M., Norton, J. E., \& Yeomans, M. R. (2016). Emulsion oil droplet size significantly affects satiety: A pre-ingestive approach. Appetite, 96, 18-24.

Li, Y., Hu, M., \& McClements, D. J. (2011). Factors affecting lipase digestibility of emulsified lipids using an in vitro digestion model: Proposal for a standardised pH-stat method. Food Chemistry, 126(2), 498505.

Maljaars, P. W. J., Peters, H. P. F., Kodde, A., Geraedts, M., Troost, F. J., Haddeman, E., \& Masclee, A. A. M. (2011). Length and site of the small intestine exposed to fat influences hunger and food intake. The British Journal of Nutrition, 106(10), 1609-15.

Maljaars, P. W. J., van der Wal, R. J. P., Wiersma, T., Peters, H. P. F., Haddeman, E., \& Masclee, A. a M. (2012). The effect of lipid droplet size on satiety and peptide secretion is intestinal site-specific. Clinical Nutrition (Edinburgh, Scotland), 31(4), 535-42.

Marquis, M., Davy, J., Cathala, B., Fang, A., \& Renard, D. (2015). Microfluidics assisted generation of innovative polysaccharide hydrogel microparticles. Carbohydrate Polymers, 116, 189-199.

Martins, E., Poncelet, D., Marquis, M., Davy, J., \& Renard, D. (2017). Monodisperse core-shell alginate (micro)-capsules with oil core generated from droplets millifluidic. Food Hydrocolloids, 63, 447-456.

Murata, Y., Sasaki, N., Miyamoto, E., \& Kawashima, S. (2000). Use of floating alginate gel beads for stomach-specific drug delivery. European Journal of Pharmaceutics and Biopharmaceutics, 50(2), 221-226.

Sams, L., Paume, J., Giallo, J., \& Carrière, F. (2016). Relevant pH and lipase for in vitro models of gastric digestion. Food \& Function, 7, 30-45.

Schroën, K., Bliznyuk, O., Muijlwijk, K., Sahin, S., \& Berton-Carabin, C. C. (2015). Microfluidic emulsification devices: from micrometer insights to large-scale food emulsion production. Current Opinion in Food Science, 3, 33-40.

Smit, H. J., Keenan, E., Kovacs, E. M. R., Wiseman, S. a, Peters, H. P. F., Mela, D. J., \& Rogers, P. J. (2011). No efficacy of processed Fabuless (Olibra) in suppressing appetite or food intake. European Journal of Clinical Nutrition, 65(1), 81-6.

Stewart, J. E., Seimon, R. V., Otto, B., Keast, R. S. J., Clifton, P. M., \& Feinle-Bisset, C. (2011). Marked differences in gustatory and gastrointestinal sensitivity to oleic acid between lean and obese men. American Journal of Clinical Nutrition, 93(4), 703-711.

van Avesaat, M., Troost, F. J., Ripken, D., Hendriks, H. F., \& Masclee, A. A. M. (2015). Ileal brake activation: macronutrient specific effects on eating behavior? International Journal of Obesity, 39(April), 235243.

Welch, I., Saunders, K., \& Read, N. W. W. (1985). Effect of ileal and intravenous infusions of fat emulsions on feeding and satiety in human volunteers. Gastroenterology, 89(6), 1293-1297.

Welch, I., Sepple, C. P., \& Read, N. W. (1988). Comparisons of the effects on satiety and eating behaviour of infusion of lipid into the different regions of the small intestine. Gut, 29, 306-311.

WHO. (2017). Obesity and overweight. Retrieved from http://www.who.int/mediacentre/factsheets/fs311/en/ 


\section{Summary}

English and Dutch summary 
In an ideal scenario, the increasing prevalence of overweight and obesity is counteracted by an effective preferably non-invasive weight management strategy. In this thesis, we targeted on physiological feedback involved in hunger regulation, via the so-called "ileal brake" mechanism. The ileal brake is activated when non-absorbed lipid metabolites are sensed in the ileum, and induce satiety. Ileal brake activation has been proven effective through direct delivery (via intubation) of lipids into the ileum. However, to be part of a non-invasive weight management strategy, the lipid should be ingested through the normal route of food intake, where it will face the efficient digestive processes of the human gastrointestinal (GI) tract, forming lipid metabolites that will be absorbed before reaching the ileum. When lipid digestion is delayed, i.e., taking place further down the small intestine, the formed metabolites can be sensed in the ileum and, the ileal brake mechanism can be activated.

In Chapter 2 we provided an overview of existing literature on food-grade microencapsulation systems that may induce satiety via delayed lipolysis. Two approaches are mostly used: protective interfaces in oil-in-water $(\mathrm{O} / \mathrm{W})$ emulsions, or structured (gelled) $\mathrm{O} / \mathrm{W}$ emulsion-based systems. These two approaches are explored in the following chapters. More specifically, we investigated systems with at least one biopolymer of indigestible nature.

The suitability of multilayered interfaces for intestinal delivery of non-absorbed lipids was investigated in emulsions in Chapter $\mathbf{3}$ based on static in vitro experiments, and in Chapter 4 based on model interfaces, with the focus on the effect of layer composition and thickness. Although multilayered interfaces improved the physical stability of emulsions during storage and in vitro gastric phase compared to simple whey protein-stabilized emulsions, during in vitro intestinal digestion, the initial lipolysis rates were similar for emulsions with different number of layers at the interface (Chapter 3). These observations were assigned to the rapid destabilisation of multilayers at the neutral intestinal $\mathrm{pH}$, as observed with interfacial tension and rheology experiments (Chapter 4). When single- and multilayered-interface structures were exposed to bile and/or lipase, the interfacial tension lowered rapidly, indicating highly efficient adsorptive behaviour of these components, displacing the previously adsorbed material (Chapter 4). All these findings led us to conclude that lipolysis cannot sufficiently be controlled by designing a protective interfacial structure in emulsions.

Therefore, in the following chapters, gelled O/W emulsion-based systems were used to delay diffusion of digestive components to the oil droplets. In Chapter 5, physically stable emulsion-alginate beads of different sizes $(\sim 0.3-1.7 \mathrm{~mm})$ and mesh sizes $(\sim 5.4-9.2 \mathrm{~nm})$ were produced. At $\mathrm{pH} 2.0$, the beads shrink slightly and are stable during in vitro gastric phase. At pH 7.0, the beads did swell and soften during the in vitro intestinal phase. This behaviour can lead to gastric protection and facilitation of digestion of the encapsulated 
emulsion in the small intestine. The specific intestinal release profile of lipid metabolites is controlled by bead and mesh size and showed a broad range of profiles. We quantitatively described this release profile with a diffusion-based model that can be used to design tailormade beads.

In Chapter 6, the dynamics of digestion processes were tested in in vitro experiments carried out at INRA (Nantes, France), using a dynamic gastrointestinal digestion system (DIDGI) with three consecutive compartments that simulate the stomach, duodenum and distal small intestine. Not only the digestion of the lipid was studied, but also the incorporation of absorbable lipid metabolites in mixed bile micelles. In free emulsions, the digestion was rapid and micellar solubilisation was not rate limiting, whereas emulsion-alginate beads retarded lipid digestion. The measurements done in this complex system confirmed the suitability of emulsion-alginate beads for distal (ileal) release.

Based on these findings, a human trial was performed to study the effect of emulsion-alginate beads on feelings of satiety and food intake as described in Chapter 7. Emulsion-alginate beads were added to a yogurt and ingested by healthy overweight subjects in a cross-over design, with as control an equicaloric mixture of non-encapsulated nutrients with similar sensory properties. Food intake was measured $2 \mathrm{~h}$ later during ad libitum pasta meal consumption, and found to be significantly reduced with $51 \pm 20 \mathrm{kcal}$ compared to the control (mean reduction of $6.2 \%$ ).

These findings are just the beginning, and we foresee many ways to optimize the release profile from emulsion-alginate beads, as discussed in Chapter 8. This chapter not only describes the technical optimization of the beads, incorporation in food and large-scale production, but also the steps that need to be taken to optimize physiological effectiveness (e.g., understanding the mechanism of action, dose-response relations and long-term effectiveness). We expect that emulsion-alginate beads are able to play a pivotal role in appetite control, and can be part of a non-invasive weight management strategy that does not burden 'patients'. 
In de ideale wereld zou de toenemende prevalentie van overgewicht en obesitas worden opgevangen door een effectieve maar bij voorkeur niet-invasieve methode om af te vallen. In dit proefschrift hebben we ons gericht op fysiologische feedback die betrokken is bij honger regulatie, de zogenaamde "ileal brake" (de rem in het ileum). De ileal brake wordt geactiveerd door het detecteren van niet-geabsorbeerde afbraakproducten van vetvertering in het ileum, en leidt tot een verzadigingsgevoel. Activering van de ileal brake is bewezen door directe intubatie van vetten in het ileum. Echter, voor een niet-invasieve methode om af te vallen, zou het vet moeten worden toegediend via de normale route van voedingsinname. Tijdens deze route ondergaat het vet de efficiënte verteringsprocessen van het menselijke maagdarmstelstel, waardoor de vetafbraakproducten vroegtijdig zullen worden geabsorbeerd en het ileum niet bereiken. Als vetvertering geremd en vertraagd wordt, en dus later in de dunne darm plaatsvindt, kunnen de vetafbraakproducten worden gedetecteerd in het ileum, en de ileal brake activeren.

In Hoofdstuk 2 is een overzicht gegeven van gepubliceerde literatuur over microincapsuleringssystemen voor voeding die een verzadigingsgevoel zouden kunnen oproepen via vertraagde vetvertering. De twee meest gebruikte aanpakken zijn: beschermende grensvlakken in olie-in-water $(\mathrm{O} / \mathrm{W})$-emulsies, of systemen gebaseerd op gestructureerde (ge-gel-eerde) O/W-emulsies. Deze twee aanpakken zijn beiden onderzocht in dit proefschrift; meer specifiek, er is altijd minimaal één onverteerbare biopolymeer gebruikt.

De geschiktheid van meerlagige grensvlakken voor het afleveren van niet-geabsorbeerde vetafbraakproducten in het ileum is onderzocht in emulsies in Hoofdstuk $\mathbf{3}$ gebaseerd op statische in vitro experimenten, en in Hoofdstuk 4 gebaseerd op model grensvlakken, met focus op het effect van laagcompositie en laagdikte. Ondanks dat meerlagige grensvlakken de fysische stabiliteit van emulsies verbeterde tijdens opslag en een in vitro maag-fase (vergeleken met simpele wei eiwit-gestabiliseerde emulsies), tijdens in vitro intestinale vertering was de initiële verteringssnelheid gelijk voor emulsies met verschillend aantal lagen (hoofdstuk 3). We verklaren deze bevinding met de snelle destabilisering van meerlagige structuren bij neutrale intestinale $\mathrm{pH}$, zoals gevonden met oppervlaktespanning en reologie experimenten (hoofdstuk 4). Wanneer enkel- en meerlagige grensvlakstructuren blootgesteld werden aan gal en/of lipase, verlaagde de oppervlaktespanning snel, wat aangeeft dat deze componenten erg efficiënt adsorberen en het initieel geadsorbeerde materiaal vervangen (hoofdstuk 4). Al deze bevindingen samen leiden tot de conclusie dat vetvertering niet voldoende kan worden geremd door beschermende grensvlakken in O/W-emulsies.

Daarom hebben we in de volgende hoofdstukken ge-gel-eerde O/W-emulsies onderzocht die de diffusie van verteringscomponenten naar de oliedruppels vertragen. In Hoofdstuk 5 zijn fysisch stabiele emulsie-alginaat bolletjes geproduceerd met verschillende grootte $(\sim 0.3-1.7$ 
$\mathrm{mm}$ ) en maasgrootte ( 5.4-9.2 nm). Bij pH 2.0 krompen de emulsie-alginaat bolletjes een beetje en waren stabiel tijdens de in vitro maag-fase. Bij pH 7.0 zwollen ze en werden zacht tijdens de in vitro intestinale fase. Dit gedrag kan leiden tot bescherming in de maag en vertering van de geincapsuleerde emulsie in de dunne darm. De specifieke intestinale afgifteprofielen (van vetafbraakproducten) kunnen worden gecontroleerd met bolletjes- en maasgrootte, en heel verschillende profielen werden gevonden. We hebben deze afgifteprofielen kwantitatief beschreven met een diffusie-gebaseerd model, dat kan worden gebruikt voor het ontwerp van op maat gemaakte emulsie-alginaat bolletjes.

In Hoofdstuk 6 hebben we dynamische verteringsprocessen onderzocht in in vitro experimenten die zijn uitgevoerd bij INRA (Nantes, France), met behulp van een dynamische maagdarm verteringssysteem (DIDGI) met drie opeenvolgende compartimenten die de maag, duodenum, en distale dunne darm simuleren. Naast de vetvertering zelf hebben we ook gekeken naar de incorporatie van absorbeerbare vetafbraakproducten in gemengde gal micellen. In vrije emulsies was de vertering snel en de oplosbaarheid in micellen niet snelheid-limiterend, terwijl de vetvertering werd vertraagd door emulsie-alginaat bolletjes. De metingen gedaan in dit complexe systeem bevestigden de geschiktheid van emulsiealginaat bolletjes voor distale (ileale) afgifte.

Gebaseerd op deze bevindingen is een humane interventiestudie uitgevoerd om het effect van emulsie-alginaat bolletjes op voedingsinname en het verzadigingsgevoel te onderzoeken, zoals beschreven in Hoofdstuk 7. Gezonde proefpersonen met overgewicht kregen een yoghurt met emulsie-alginaat bolletjes in een cross-over design met als controle een yoghurt met niet-geincapsuleerde nutriënten van gelijke calorische waarde en sensorische eigenschappen. Voedingsinname werd twee uur later gemeten tijdens een onbeperkte pasta maaltijd, en was significant lager (51 $\pm 20 \mathrm{kcal}$ ) vergeleken met de controle (gemiddelde reductie van $6.2 \%)$.

Deze bevindingen zijn slechts het begin, we voorzien verschillende manieren om het afgifteprofiel uit emulsie-alginaat bolletjes te optimaliseren, zoals beschreven in Hoofdstuk 8. Dit hoofdstuk beschrijft niet alleen de technische optimalisatie van de bolletjes, incorporatie in voeding, en productie op grotere schaal, maar ook de stappen die nodig zijn om de fysiologische effectiviteit te optimaliseren (bv. het begrijpen van het mechanisme, dosering-response relaties, en langere termijn effectiviteit). We verwachten dat emulsiealginaat bolletjes een belangrijke rol gaan spelen in de regulatie van eetlust, en deel kunnen uitmaken van een niet-invasieve methode om af te vallen, die een lage belasting voor 'patiënten' heeft. 



\section{Acknowledgements}

Ik ben enorm blij en trots dat ik op het punt aangekomen ben om het dankwoord van dit proefschrift te schrijven. Er zijn een heel aantal mensen die ik ontzettend dankbaar ben voor hun bijdrage (aan kennis, aan vaardigheden, en aan gezelligheid) de afgelopen vier $\mathrm{PhD}$ jaren.

To start with, I would like to truly thank my FPE-supervisors Claire and Karin, who have supported me throughout my PhD. I have learned so much from you, regarding content, scientific writing, and research skills in the broadest way of the definition! I would also like to thank Freddy, Ad and Renko for their supervision over distance.

Dear FPE PhDs, thanks for the nice coffee breaks, lunch walks, sport activities, drinks, parties, $\mathrm{PhD}$ trips, and of course for the inspiring discussions. Special thanks to Kelly and Patricia for being great roomies, and also to Lisa for the last year. Many thanks to the technicians: Martin for being my 'partner' in the FPP practical course; Maurice for your help with $\mathrm{pH}$ stat, building setups and microscopy; Jarno for the cooperation in CLSM and AAS; and Jos for always knowing an answer or solution to any kind of question or problem. Furthermore, thanks to Jan-Eise for the discussions about modelling and your help with my design diagrams.

I worked in several environments during my $\mathrm{PhD}$, which I greatly enjoyed due to the warm welcome, helpfulness, and great collaboration! So many many thanks to my 'temporary' colleagues at INRA (Nantes, FR), at MDL (Maastricht), and at PCC (Wageningen).

I also really liked supervising 7 MSc students; thanks, especially for raising many questions, from which I have learned a lot. Besides, many thanks to my other co-authors, I enjoyed our collaborations and I have learned a lot from all the discussions we have had.

Verder wil ik graag mijn (schoon)familie en vrienden heel erg bedanken voor alle fijne momenten samen de afgelopen jaren. Ik heb enorm veel steun en begrip gevoeld op minder makkelijke momenten. Ondanks het feit dat ik het hier kort houd en geen namen noem, is mijn dank aan alle betrokkenen zeer groot! Zonder jullie zou het onmogelijk zijn geweest om zo gelukkig door mijn PhD heen te komen. In het bijzonder wil ik mijn ouders bedanken, voor al het optimisme, de steun, en de opvoeding tot de persoon die ik nu ben.

Last but not least, ben ik Michiel enorm dankbaar voor zijn liefde, begrip, en onvoorwaardelijke support. Ook ben ik buitengewoon dankbaar voor de komst van onze prachtige lieve zoon Lucas. Alle geluk en liefde die hij met zich mee brengt gaven een erg positieve draai aan de laatste stadia van mijn PhD. Ik hou onwijs veel van jullie. 

Author

About the author 


\section{Curriculum Vitae}

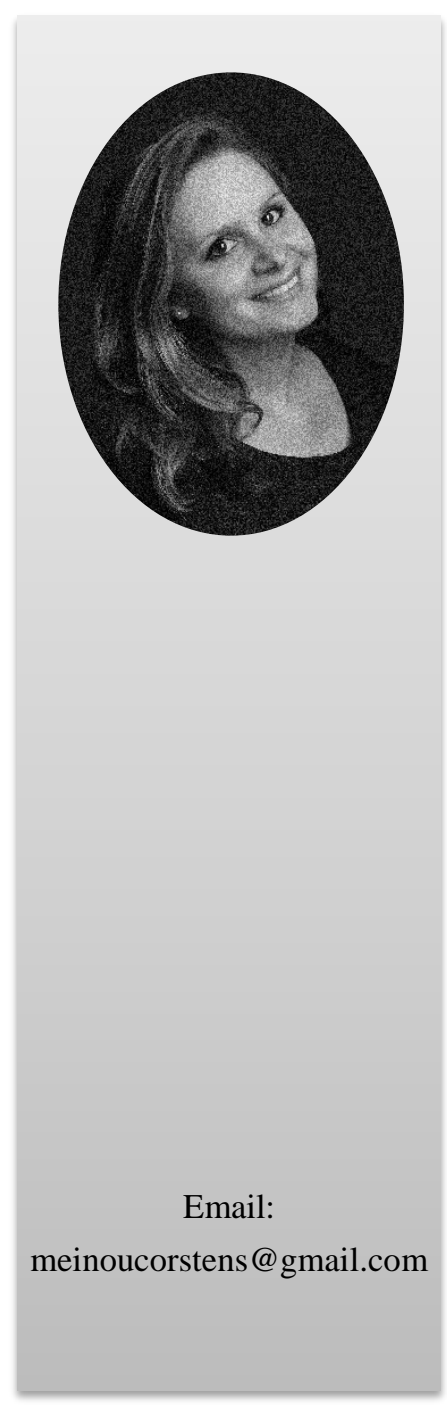

Meinou Noëlle Corstens was born December 26, 1989 in Breda, The Netherlands. She took secondary school at 2 College Cobbenhagen where she obtained her VWO diploma in 2008 with a major in Nature and Health.

In the same year, she started her study Nutrition \& Health at Wageningen University, with a minor in Food Technology, and a minor thesis at the laboratory of Human Nutrition. In 2011, she started the master Food Technology with specialisation Ingredient Functionality, with a major thesis in the group Food Physics on The effect of biopolymers on the texture of sorbet. During her internship at Unilever R\&D Vlaardingen, she investigated The role of emulsifiers in formation and stabilization of foam. She graduated with distinction during both her BSc (2011) and MSc (2013) studies.

Meinou continued working as $\mathrm{PhD}$ candidate on the project Encapsulation of lipids for targeted release in the gastro-intestinal tract at the laboratory of Food Process Engineering at Wageningen University and Research, in collaboration with the department of Internal Medicine at Maastricht University and Medical Centre+, and established an exchange with the laboratory of Biopolymères Interactions Assemblages at INRA (Nantes, France). This thesis entitled Encapsulation of lipids to delay lipolysis and reduce food intake is the result of that PhD project. 


\section{List of publications}

Corstens, M.N., Berton-Carabin, C.C., de Vries, R., Troost, F.J., Masclee, A.A.M., \& Schroën, K. (2017) Food-grade Micro-encapsulation Systems that May Induce Satiety via Delayed Lipolysis: A Review. Critical Reviews in Food Science and Nutrition 57(10):22182244.

Corstens, M.N., Berton-Carabin, C.C., Kester, A, Fokkink, R, van der Broek, J.M., de Vries, R., Troost, F.J., Masclee, A.A.M., \& Schroën, K. (2017) Destabilization of multilayered interfaces in digestive conditions limits their ability to prevent lipolysis in emulsions. Food Structure 12:54-63.

Corstens, M.N., Osorio Caltenco, L.A., de Vries, R., Schroën, K., Berton-Carabin, C.C. (2017) Interfacial behaviour of biopolymer multilayers: influence of in vitro digestive conditions. Colloids and Surfaces B: Biointerfaces 153:199-207.

Corstens M.N., Berton-Carabin C.C., Elichiry-Ortiz P.T., Hol K., Troost F.J., Masclee A.A.M., Schroën K. (2017) Emulsion-alginate beads designed to control in vitro intestinal lipolysis: Towards appetite-control. Journal of Functional Foods 34:319-28.

Corstens, M.N., Berton-Carabin, C.C., Schroën, K., Viau, M., Meynier, A. Emulsion encapsulation in calcium-alginate beads retards lipolysis during dynamic in vitro digestion. Submitted.

Corstens, M.N., Troost, F.J., Alleleyn, A.M.E., Klaassen, T., Berton-Carabin, C.C., Schroën, K., Masclee, A.A.M. Encapsulation of lipid as emulsion-alginate beads reduces food intake: a randomized placebo-controlled cross-over trial in overweight adults. Submitted.

\section{Book chapter:}

Schröder, A., Corstens, M., Ho, K., Schroën, K., Berton-Carabin, C. (in press). Pickering emulsions. In S. Roohinejad, R. Greiner, I. Oey, \& J. Wen (Eds.), Emulsion-based systems for delivery of food active compounds: Formation, application, health and safety. John Wiley \& Sons, Ltd. 


\section{Overview of completed training activities}

\begin{tabular}{|c|c|c|}
\hline Discipline specific activities & & \\
\hline \multicolumn{3}{|l|}{ Workshops } \\
\hline Workshop fat digestion, Lorentz institute & Leiden, NL & 2013 \\
\hline Winter school in Physical Chemistry & Han-Sur-Lesse, BE & 2014 \\
\hline Training period dynamic in vitro lipolysis, INRA & Nantes, FR & 2016 \\
\hline eBROK course, CTCM & Maastricht, NL & 2017 \\
\hline \multicolumn{3}{|l|}{ Conferences } \\
\hline $3^{\text {rd }}$ International Conference on Food Digestion & Wageningen, NL & 2014 \\
\hline $1^{\text {st }}$ Food Structure \& Functionality & Amsterdam, NL & 2014 \\
\hline $4^{\text {th }}$ International Conference on Food Digestion & Naples, IT & 2015 \\
\hline $16^{\text {th }}$ Food Colloid Conference & Wageningen, NL & 2016 \\
\hline \multicolumn{3}{|l|}{ General courses } \\
\hline VLAG PhD week & Baarlo, NL & 2014 \\
\hline Competence assessment & Wageningen, NL & 2014 \\
\hline Scientific writing & Wageningen, NL & 2014 \\
\hline Afstudeervak begeleiden en organiseren & Wageningen, NL & 2015 \\
\hline PhD Workshop Carousel & Wageningen, NL & 2015 \\
\hline Techniques for Writing and Presenting a Scientific Paper & Wageningen, NL & 2016 \\
\hline Career perspectives (CCP) & Wageningen, NL & 2017 \\
\hline \multicolumn{3}{|l|}{ Optional activities } \\
\hline FPE symposia & Wageningen, NL & $2014-2016$ \\
\hline FPE-group theme meetings & Wageningen, NL & 2013-2017 \\
\hline Project meetings Maastricht & Maastricht, NL & $2013-2017$ \\
\hline Excursion Chile and Brazil & $\mathrm{CH}$ and $\mathrm{BR}$ & 2014 \\
\hline Excursion Switzerland and Germany & SW and GE & 2016 \\
\hline
\end{tabular}


The research described in this thesis was financially supported by the Graduate School VLAG, and was a cooperation between the laboratory of Food Process Engineering at Wageningen University and Research and the department of Internal Medicine at Maastricht University and Medical Centre+.

This thesis is printed by ProefschriftMaken in 150 copies.

Cover design: Jasper Kalle. 


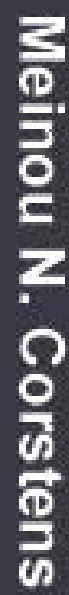

$\frac{\mathfrak{d}}{\infty}$ 